Portland State University

PDXScholar

$1-1-2010$

\title{
The MicroPIVOT : an Integrated Particle Image Velocimeter and Optical Tweezers Instrument for Microscale Investigations
}

Nathalie Neve de Mevergnies

Portland State University

Follow this and additional works at: https://pdxscholar.library.pdx.edu/open_access_etds Let us know how access to this document benefits you.

\section{Recommended Citation}

Neve de Mevergnies, Nathalie, "The MicroPIVOT : an Integrated Particle Image Velocimeter and Optical Tweezers Instrument for Microscale Investigations" (2010). Dissertations and Theses. Paper 31.

https://doi.org/10.15760/etd.31

This Dissertation is brought to you for free and open access. It has been accepted for inclusion in Dissertations and Theses by an authorized administrator of PDXScholar. Please contact us if we can make this document more accessible: pdxscholar@pdx.edu. 
The MicroPIVOT

An Integrated Particle Image Velocimeter and Optical Tweezers Instrument

for Microscale Investigations

by

Nathalie Nève de Mévergnies

A dissertation submitted in partial fulfillment of the requirements for the degree of

\author{
Doctor of Philosophy \\ in \\ Mechanical Engineering \\ Dissertation Committee: \\ Derek Tretheway, Chair \\ Yongkang Chen \\ Sean Kohles \\ Mark Weislogel \\ Randy Zelick \\ Gwynn Johnson
}

Portland State University

(C)2010 
This dissertation describes the development of a device capable of suspending a microscale object in a controlled flow. The $\mu$ PIVOT is a system integrating two laserbased techniques: micron particle image velocimetry ( $\mu \mathrm{PIV}$ ) and optical tweezers (OT). The OT allows the suspension and manipulation of micron-sized objects such as microspheres or biological cells. $\mu$ PIV provides imaging of the suspended object and velocity measurements from which fluid induced stresses can be determined. Using this device, we measured fluid velocities around an optically suspended polystyrene microsphere (an experimental first) and studied the interaction between two particles suspended in a uniform flow. The results were consistent with theoretical low Reynolds number, Newtonian flow predictions. Additionally, we analyzed a single cell's mechanical response to a controlled and measurable multiaxial external force (fluid flow) without the cell being physically attached to a surface. The cell's mechanical response was monitored by observing its morphology and measuring its deformation. The results show significant deformations of optically suspended cells at substantially smaller stresses than previously reported and demonstrate the opportunity to optically distinguish a cell by its trapping efficiency. These initial applications of the $\mu$ PIVOT demonstrate the potential of this unique device as a research tool for novel studies in the fields of fluid/particle(s) interactions, non-Newtonian fluid mechanics, and single cell biomechanics. 


\section{ACKNOWLEDGMENTS}

It is a pleasure to convey my gratitude to those who made this project and dissertation possible. I would first like to express the deepest appreciation to my advisor Dr. Derek Tretheway for his excellent guidance, patience, trust, and providing me with an excellent research environment. I would like to thank my committee members: Dr. Sean Kohles for his precious help, advice, and ideas at the beginning of the project, Dr. Yongkang Chen and Dr. Mark Weislogel for their unlimited support, valuable insight and kind friendship these past four years, and Dr. Randy Zelick and Dr. Gwynn Johnson for their constructive comments on this dissertation.

I would also like to thank my lab mates Jeremiah Zimmerman who was there every time I needed his priceless help, and James Lingwood for his cooperation in the design of the instrumentation. I am much indebted to the Mechanical and Material Engineering departmental team, especially the department secretary and dear friend Molly Meyer as well as Chuck for his invaluable assistance in the machine shop. I am grateful to Dr. Shelley Winn, from the Oregon Health and Science University who has provided all the biological cell material and an inestimable expertise.

My parents deserve very special recognition for their inexhaustible confidence in me and for supporting me whatever choices I made throughout all these years. But most of all, I would like to thank them for sharing their insatiable curiosity about the world and teaching me how to reach happiness. 
Finally, words fail me to fully express my appreciation to my husband Michaël

whose dedication, love, complicity and daily help have carried me higher than I could have imagined, and allowed me to pursue this project from start to finish. 
ABSTRACT

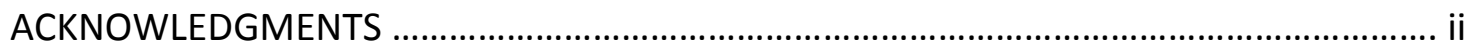

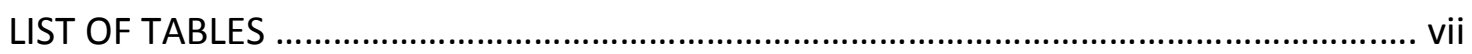

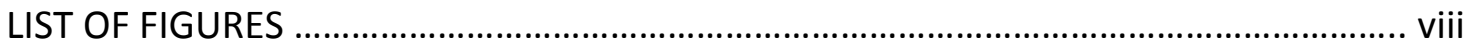

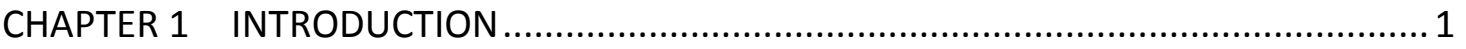

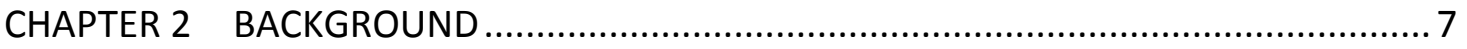

2.1 CELLS, STRESSES AND MECHANOTRANSDUCTION …................................ 7

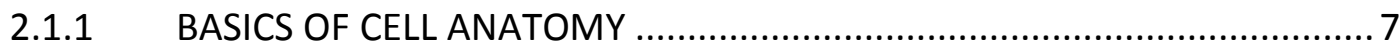

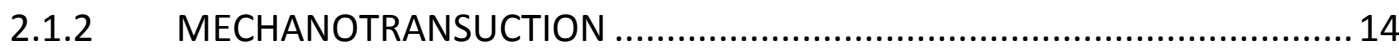

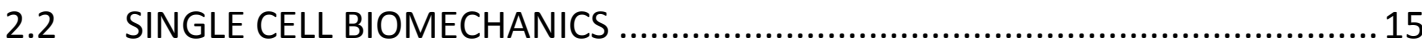

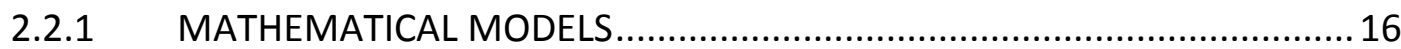

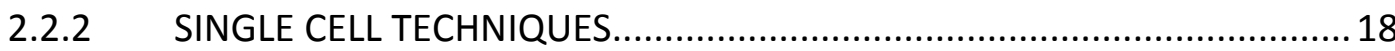

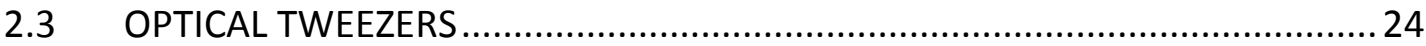

2.3.1 THEORY OF OPTICAL TWEEZERS IN THE MIE REGIME ......................... 25

2.3.2 CALIBRATION TECHNIIQUES: TRAP STIFFNESS DETERMINATION ...........33

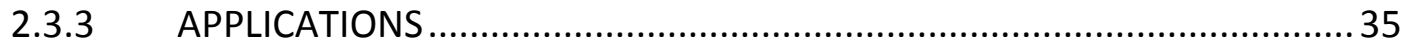

2.4 MICRON RESOLUTION PARTICLE IMAGE VELOCIMETRY.................................. 36

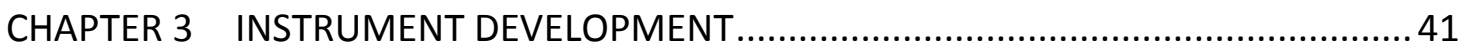

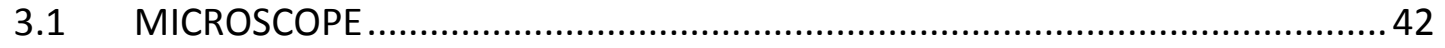

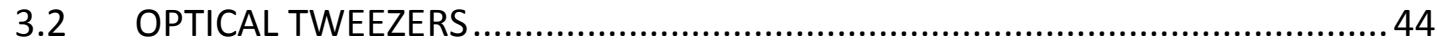

3.3 MICRON RESOLUTION PARTICLE IMAGE VELOCIMETRY ...............................51

$3.4 \quad$ AUTOMATION OF THE SYSTEM ............................................................ 52

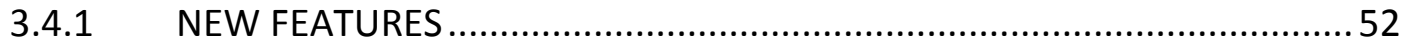

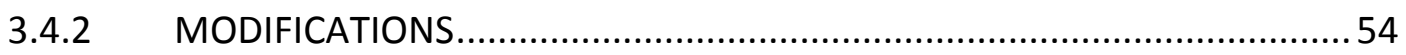

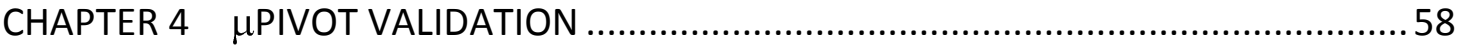

4.1 CHARACTERIZATION OF THE OPTICAL TWEEZERS .....................................58

4.1.1 RANGE OF MOTION AT THE SAMPLE .................................................. 58

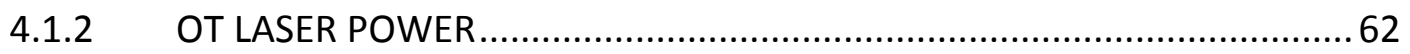

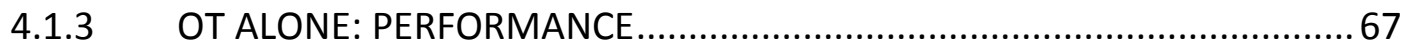

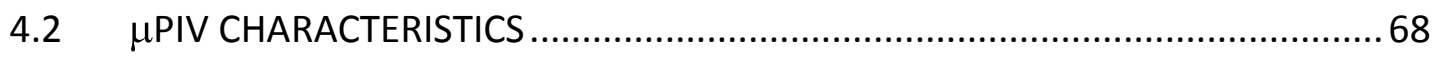

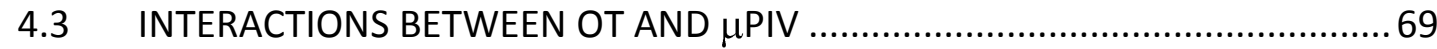

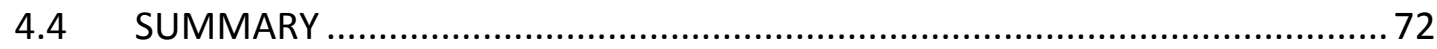

CHAPTER 5 FLOW AROUND A SINGLE FREELY SUSPENDED SPHERE ........................73

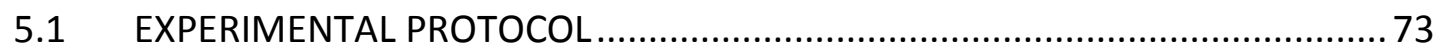

5.1.1 STATIONARY SPHERE IN A GRAVITY DRIVEN MICROCHANNEL FLOW .... 73 
5.1.2 STATIONARY SPHERE IN A UNIFORM FLOW .......................................... 76

5.1 .3 STATIONARY SPHERE IN AN EXTENSIONAL FLOW ................................. 78

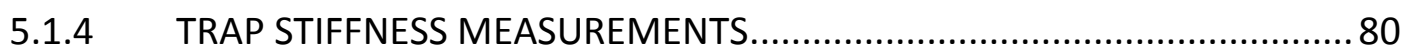

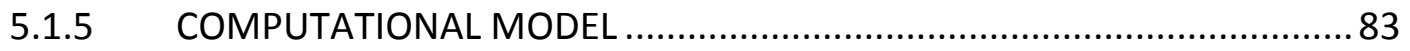

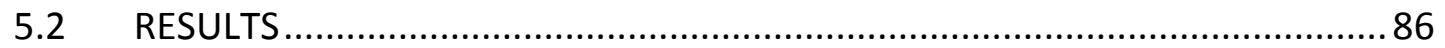

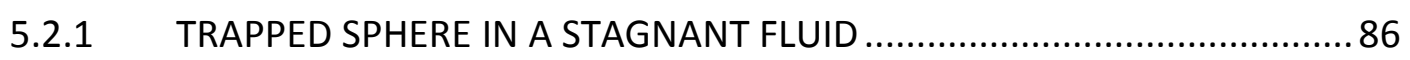

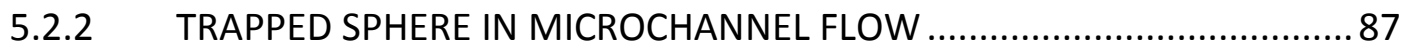

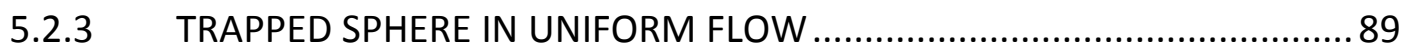

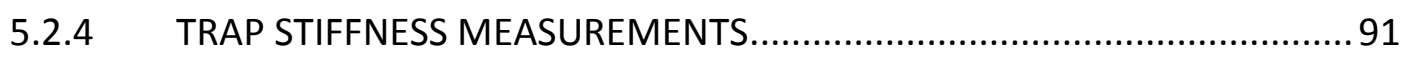

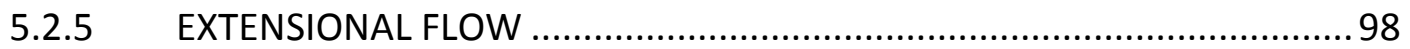

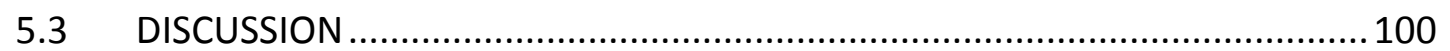

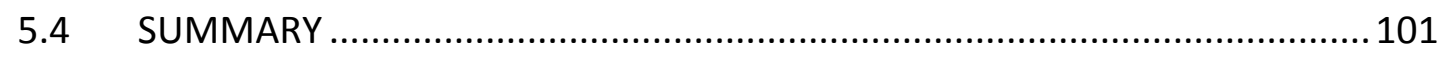

CHAPTER 6 UNIFORM FLOW AROUND TWO SUSPENDED SPHERES ...................... 102

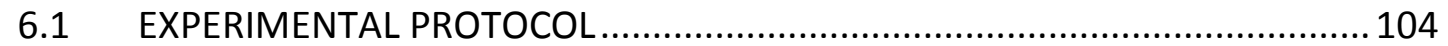

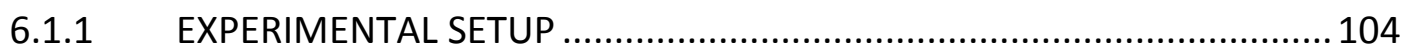

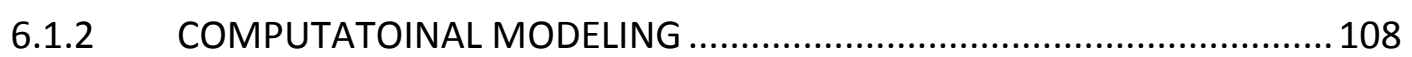

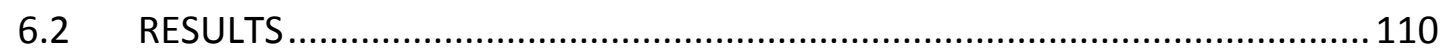

6.2.1 DRAG FORCE OF TWO SPHERES IN A UNIFORM FLOW ....................... 110

6.2.2 FLUID VELOCITY AROUND TWO SPHERES PARALLEL TO THE FLOW ....118

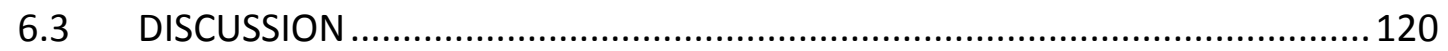

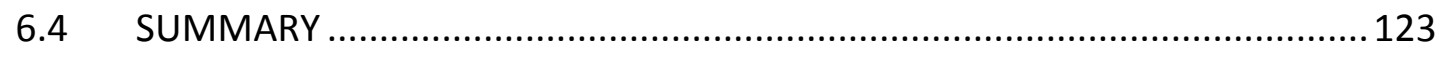

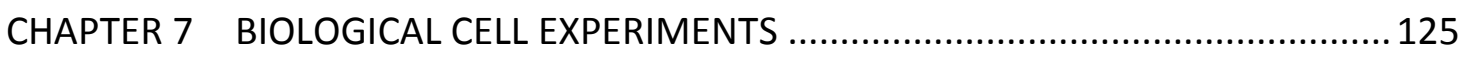

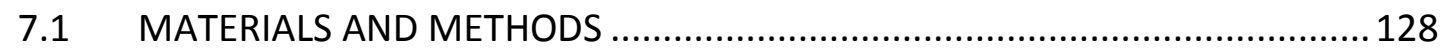

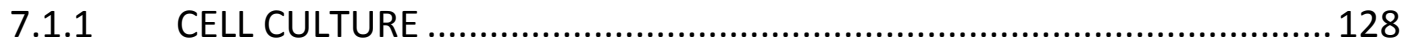

7.1.2 CELL RESERVOIRS AND MICROFLUIDIC DEVICE FABRICATION ..............130

7.2 EXPERIMENTAL PROTOCOLS ............................................................... 133

7.2.1 STATIC ENVIRONMENT: OT-INDUCED STRESS AND HYDROSTATIC-

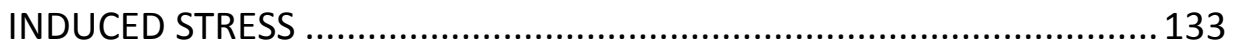

7.2.2 DYNAMIC ENVIRONMENT: UNIFORM AND EXTENSIONAL FLOWS ...... 135

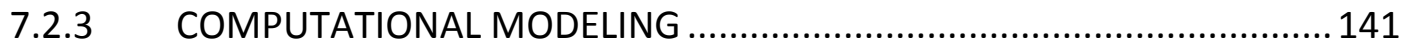

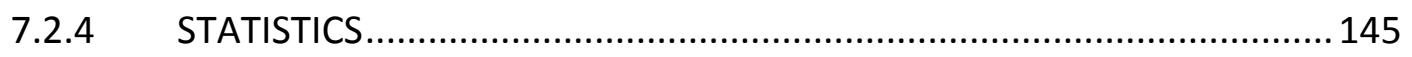

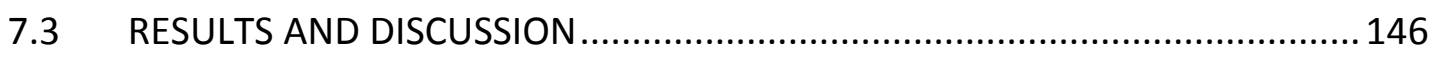

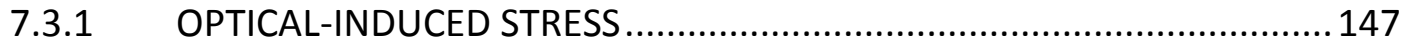

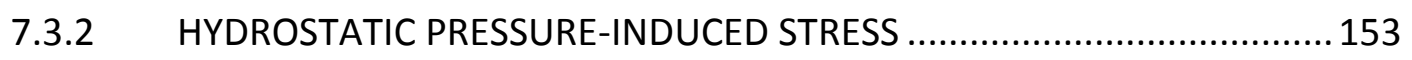

7.3.3 HYDRODYNAMIC-INDUCED STRESS: UNIFORM FLOW ........................ 155

7.3.4 EFFECTS OF CELL ELONGATION AND CILIA ON DRAG FORCE:

7.3.5 HYDRODYNAMIC-INDUCED STRESS: EXTENSIONAL FLOW ................... 171

7.4 COMMENT ON RELEVANCE TO MECHANOTRANSDUCTION ........................ 175

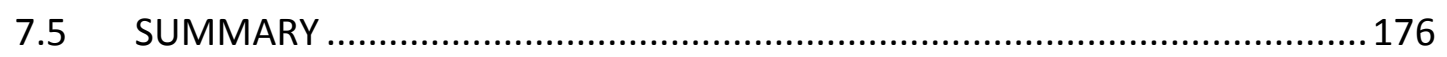




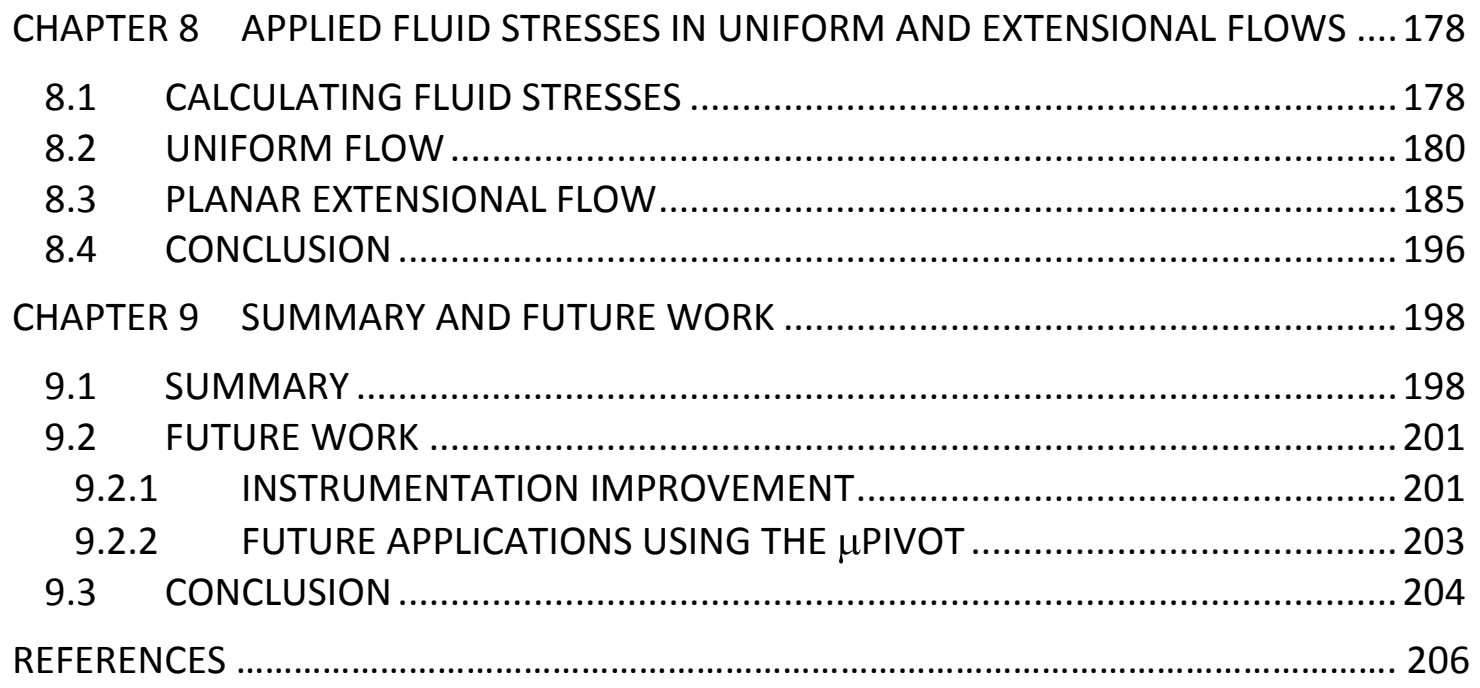




\section{LIST OF TABLES}

Table 1: List of mathematical models used for different experimental techniques with the resulting cell material properties. The right column indicates the references from which the data was taken: A: [Alexopoulos, 2003], B: [Alexopoulos, 2005], C: [Darling, 2006], D: [Guilak, 2005], E: [Jones, 1999], F: [Koay, 2003], G: [Leipzig, 2005], H: [Shieh, 2006], I: [Trickey, 2000], J: [Zhang, 2008].

Table 2: Theoretical results from Stimson and Jeffery [Stimson, 1926] ........................ 110

Table 3: Average modified trap stiffness for chondroblasts split into different groups. No significant difference due to surface roughness, cilia size or cell concentration... 162 


\section{LIST OF FIGURES}

Figure 1: Bone model before and after a long spaceflight (Images from the European

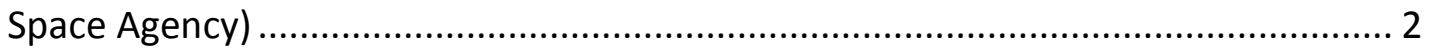

Figure 2: Schematic of cellular response to a mechanical perturbation [Shieh, 2003]...... 3

Figure 3: Schematic of a eukaryotic cell [http://www.columbia.edu/cu/biology/courses].

Figure 4: Cytoskeleton of a cow endothelial cell where actin filaments are stained in red and microtubules in yellow. The nucleus is stained in blue (Image from Molecular probes)

Figure 5: Anatomy of bone (www.web-books.com). 11

Figure 6: Picture of articular cartilage stained with Hematoxylin and Eosin (image from the University of Western Australia- School of Anatomy and Human Biology)....... 13

Figure 7: Micropipette aspiration of hMSCs at room temperature. Images (A-D) are displayed at time $t=1 \mathrm{~s}, 15 \mathrm{~s}, 100 \mathrm{~s}$ and $200 \mathrm{~s}$ after the application of step aspiration pressure, respectively [Tan, 2008]. 18

Figure 8: Schematic of AFM cell indentation experiment [Costa, 2006] ......................... 21

Figure 9: Qualitative view of the trapping of a dielectric sphere ................................. 26

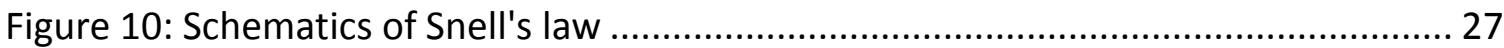

Figure 11: Geometry for calculating the force due to the scattering of a single incident ray of power $\mathrm{P}$ by a dielectric sphere, showing the reflected ray $\mathrm{PR}$ and infinite set of refracted rays $P T^{2} R^{n}$

Figure 12: Simplified cross-correlation schematics: nanoparticles move with the flow and can be visualized at two different times in order to obtain the flow direction and velocity field in the entire field of view.

Figure 13: Effect of ensemble correlation: A) results with conventional correlation for one of the PIV recording pairs; B) results with ensemble correlation for over 100

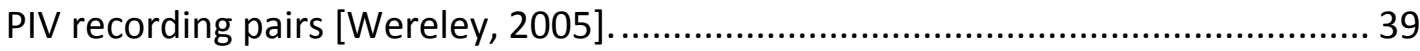

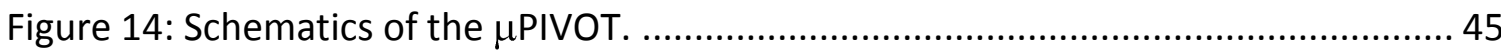

Figure 15: Picture of the $\mu$ PIVOT set up. The red lines indicate the path taken by the OT laser beam 45

Figure 16: Transmission of p-polarized light and reflection of s-polarized light through a cube beam splitter (image from Newport Corp).

Figure 17: Demonstration of trap movement through movement of a mobile lens.

Translation of the mobile lens axially or transversally causes a displacement of the focus point at the sample. 
Figure 18: Key wavelengths (in $\mathrm{nm}$ ) of the $\mu$ PIVOT system. The stripe colors indicate the transmission through the filters. Any light beam having a wavelength in the horizontal stripes zone goes through the low pass filter, but reflects off the high pass filter. Inversely, any light beam having a wavelength in the vertical stripes zone goes through the high pass filter but reflects off the low pass filter. If in the grid zone, the light beam goes through both of the filters.

Figure 19: Close-up pictures of the current $\mu$ PIVOT configuration. A) addition of beam elevators and aluminum blocks to raise the OT optical path. B) $\mu$ PIV entry port is below the OT entry port.

Figure 20: Solid Works representation of the elevation of the OT system: Gray features represent the back of the microscope and the translation stages. Colored components are the customized-built features added: Aluminum blocks (dark blue) were positioned under the translation stages, aluminum plates were added to position the lenses closer to the fixed lens (light blue), a holder (yellow) was designed to hold the beam splitting cube and the fixed lens inside the microscope.

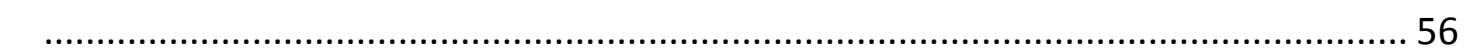

Figure 21: Picture of the modified setup. 56

Figure 22: Schematic of the automated $\mu$ PIVOT device and the current optical arrangement. The OT laser goes through different optical components including beam splitting cubes to allow the existence of two lasers of opposite polarization. The position of their focus point can be controlled independently using two mobile lenses that translate in any direction with automated control $(0.1 \mu \mathrm{m}$ resolution). They can both operate simultaneously or individually, using shutters placed in the laser beams paths. Both of the OT laser beams reunite through a second beam splitting cube before entering the microscope through an optical port, while the $\mu$ PIV laser enters it through a second port via an optical cable.

Figure 23: Reflection of the lasers of trap1 and 2 on the coverslip. The dissimilar morphologies are due to the difference in their polarization. 59

Figure 24: Trap 1 and 2 when in focus (in the sample, above the coverslip).

Figure 25: Schematic of the movement range of each trap at the sample in all three directions. The red spot indicates the mobile lens home position. In the focus plane, only a range equal to the field of view is necessary, but the possibilities of the instrumentation outrun this constraint.

Figure 26: $\mu$ PIVOT system. The circled letters represent the different positions of power measurements: $\mathrm{H} \rightarrow$ laser Head, B1 and B2 $\rightarrow$ traps 1 and 2 after Beam splitting 
cube, $\mathrm{M} \rightarrow$ before entering the Microscope, $\mathrm{O} \rightarrow$ before the Objective lens, $\mathrm{S} \rightarrow$ at the Sample.

Figure 27: Variation of laser power with position when going through the optical path.

The letters in parenthesis refer to Figure 26.................................................... 63

Figure 28: Trap power variation with half-wave plate position. ................................... 65

Figure 29: Trap 1 time dependence of the laser power measured after the first beam

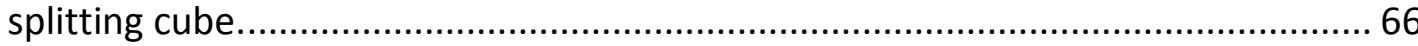

Figure 30: Power fluctuation with time for different diode currents. Measurements taken for both traps before entering the microscope (at $\mathrm{M})$...............................67 67

Figure 31: (a) Trap stiffness as a function of the applied laser power for trapped polystyrene microspheres. (b) Trap stiffness as a function of sphere diameter for a constant laser power of $0.48 \mathrm{~W}$. Stiffness values were determined from linear curve fits of the drag force versus microsphere displacement experimental data. Drag force calculations accounted for channel wall proximity. 68

Figure 32: Laminar flow velocity profiles acquired with $\mu \mathrm{PIV}$. The near wall velocity resolution is $436 \mathrm{~nm}$. The no-slip condition can be observed as the velocity decreases (vector length $=$ magnitude) to 0 at the wall.

Figure 33: Response of $\mu$ PIV nanoparticles to the applied trap laser power (measured at the microscope entrance). The number of trapped nanoparticles (bright spot) increases with trap power within (a) a static fluid and (b) a fluid moving at constant velocity (shown by the streaklines).

Figure 34: A 3-D simplified sketch of the single sphere in flow experiment. (a) A particle trapped in the middle of a straight channel with laminar flow (Reynolds number $=10^{-2}$ ). (b) A sphere held stationary in between two coverslips moving at a constant velocity. The sphere is more than $1 \mathrm{~mm}$ below the top coverslip.

Figure 35: A PDMS chip bonded to a glass coverslip with four $50 \times 500 \mu \mathrm{m}$ straight channels. The experiments were conducted in a single channel.

Figure 36: Schematic of a sphere trapped at the stagnation point of a cross-junctional

flow. The bold arrows represent the flow directions. ......................................... 78

Figure 37:(a) Cross-junction silicone mold. (b) Microfluidic chip................................... 78

Figure 38: Schematic of trapped sphere displaced from its equilibrium position without and with uniform flow. 81

Figure 39: Basic steps for sphere position determination using the software Spotlight-8.

(a) Raw image. (b) Standard threshold to obtain a white continuous border around the sphere. (c) Morphological hole fill, to obtain a black and white image of the sphere. (d) Center tracking to determine the center of the white object. 
Figure 40: A 3-D representation of the computational flow domain. (a) Uniform flow half-space (b) Gravity driven flow quadrant. The color scales represent the magnitude of the velocity in the general flow direction, in $\mu \mathrm{m} / \mathrm{s}$ ( $x$ axis)....

Figure 41: A trapped polystyrene microsphere among a static solution of nanoparticles at a laser power of $0.49 \mathrm{~W}$. The $\mu \mathrm{PIV}$ laser is on and therefore the nanoparticles fluoresce. No trapping of the nanoparticles is observed due to the shield created by the size of the microsphere. 86

Figure 42: Comparison between the experimental velocity field and the computational prediction for a stationary sphere held in a gravity driven flow. (a) $\mu$ PIV 2-D velocity field around a trapped $28 \mu \mathrm{m}$ diameter polystyrene microsphere held in a straight flow. (b) Model simulation of the experimental flow conditions 88

Figure 43: Comparison between measured (points) and predicted (line) velocities for a $28 \mu \mathrm{m}$ sphere in a gravity driven flow with a maximum plane velocity of $460 \mu \mathrm{m} / \mathrm{s} .89$

Figure 44: Quantitative comparison between measured velocities (points), computationally predicted velocities (line), and theoretical velocities without wall effects (dashed line). The inflow and outflow axis (- and $+x$ axis respectively) is normalized with the sphere radius, a, and the velocity is normalized with the automated stage velocity. (a) A $21.8 \mu \mathrm{m}$ sphere in a uniform flow at different velocities. (b) Different diameter microspheres with a constant uniform flow of 200 $\mu \mathrm{m} / \mathrm{s}$.

Figure 45: Trap stiffness dependence with the sphere diameter. (a) drag force versus sphere displacement curves for different diameter spheres. The slope of each curve represents the trap stiffness for the corresponding sphere. (b) When the drag force is multiplied by the sphere diameter, the curves all collapse into a single curve.... 92 Figure 46: Trap stiffness versus sphere diameter measured in uniform flow experiments with an OT power of $35 \mathrm{~mW}$. Experiments made before and after the modifications made to the $\mu$ PIVOT setup are consistent and show good agreement with theory.

Figure 47: Potential wells for different sphere diameters 96

Figure 48: (a) Drag force versus displacement for an $11 \mu \mathrm{m}$ sphere at different distances from the bottom coverslip. (b) Resulting trap stiffness as a function of distance from the wall.

Figure 49: (a) Velocity flow field surrounding an analogous cell (20.6 $\mu \mathrm{m}$ diameter polystyrene microsphere) as measured with micron-resolution particle image velocimetry. (b) zoom in 
Figure 50: Sketch of the different configurations. The leading sphere, at the center of the axis was held stationary while the trailing sphere was positioned at various distances from the leading sphere (up to 5 diameters away), with their centerline forming various angles from the flow axis. 105

Figure 51: Schematic of the two spheres experiment. When the automated stage moves at constant velocity, the flow induces a displacement of each of the trapped spheres. 106

Figure 52: (a) Computational model of flow around two spheres. In this configuration, $d=3$ and $\theta=0$. The flow is going from left to right. (b) $x$-y plane at $z=50 \mu \mathrm{m}$, going through the center of the spheres. The flow also comes from the left. The meshing was refined near the spheres. 109

Figure 53: Computational model results for normalized distances from 1 to 5 and angles at 0, 45 and 90 degrees. The images are taken in the $x-y$ plane at $z=50 \mu \mathrm{m}$ (going through the center of the spheres). Flow velocities range from 0 (dark blue, at the spheres surface) to the free stream velocity of $200 \mu \mathrm{m} / \mathrm{s}$ (dark red far away from the spheres).

Figure 54: Normalized drag force as a function of normalized distance between the microspheres when their centerline is parallel to the flow axis $\left(\theta=0^{\circ}\right)$. 113

Figure 55: Normalized drag force as a function of normalized distance between the microspheres for $\theta=0^{\circ}$. The stiffness of the trap trapping the trailing sphere has been lowered by $10 \%$.

Figure 56: Normalized drag force as a function of normalized distance between the microspheres for angles of $\theta=45^{\circ}$ (left) and $\theta=90^{\circ}$ (right). The stiffness of the trap trapping the trailing sphere has been lowered by $10 \%$.

Figure 57: Experimental (points) and computational (lines) results for normalized drag force of the leading sphere as a function of normalized distance between the microspheres for 0, 45 and 90 degree angles. As the centerline goes from parallel to the flow to perpendicular to the flow, the drag force increases and reaches the undisturbed drag force faster.

Figure 58: Comparison between the experimental velocity field and the computational prediction for two stationary spheres held at a normalized distance of 1.53 from each other in a uniform flow. (a) $\mu$ PIV 2-D velocity field around two trapped polystyrene microspheres (average diameter $2 a=27.6 \mu \mathrm{m}$ ) held in a uniform flow.

(b) Model simulation of the experimental flow conditions. 118

Figure 59: Comparison between measured (points) and predicted (line) velocities for two spheres (average diameter of $27.55 \mu \mathrm{m}$ ) in a $350 \mu \mathrm{m} / \mathrm{s}$ uniform flow. 119 
Figure 60: (a) Two spheres held at 45 degrees angle from horizontal axis in a stagnant flow. (b) The same spheres are shown when under a $200 \mu \mathrm{m} / \mathrm{s}$ uniform flow (from left to right). The leading sphere is shifted away from the coverslip while the trailing sphere is slightly shifted towards the coverslip.

Figure 61: Spheres rotation rate as a function of distance between them for different angles $\theta$.

Figure 62: Rotational movements of the two spheres for $\theta=90^{\circ}$. This movement induces recirculation areas and stagnation points (dotted circles) that are not observed in the computational model.

Figure 63: Attached cells: on the left osteoblasts, on the right chondroblasts (P1). Cells are approximately $15 \mu \mathrm{m}$ in diameter.

Figure 64: Microfluidic chip design (a) Cell reservoir used for static experiments and uniform flow experiments $(30 \mathrm{~mm} \times 10 \mathrm{~mm}$ ). (b) Cell reservoir used for hydrostatic experiments. The input is set at different heights while the output is closed....... 131

Figure 65: Silicone mold of the cross-junction channel. The channels are $50 \mu \mathrm{m}$ deep $\mathrm{x}$ $500 \mu \mathrm{m}$ wide. White arrows show the directions of the flow.

Figure 66: (a) Microfluidic chip of the cross-junction. (b) Detailed picture of the first version of the cross-junction channel. The red arrows represent the directions of the flow.

Figure 67: Pictures of a $17 \mu \mathrm{m}$ diameter chondroblast trapped with OT and undergoing different uniform flow velocities. The cell is shifted to the right due to the force of the flow coming from the left. The vertical line indicates the center of the cell when in a static environment. 136

Figure 68: Image processing steps to determine the cell's position.

Figure 69: Sketch of a pseudo-planar extensional flow. The bottom picture is the undisturbed velocity field of a cross-junction flow at the stagnation point. Velocities are measured with our $\mu$ PIV system.

Figure 70: A 3-D representation of the computational flow half-space domain for uniform flow around a: (a) 50\% elongated sphere in the $x$-direction (flow direction). (b) $50 \%$ elongated sphere in the $y$-direction. (c) $50 \%$ elongated sphere in the $z-$ direction. The arrows indicate the direction of the flow.

Figure 71: Modeling of the cell with six long protrusions.

Figure 72: Modeling of the cell with twenty-six small protrusions. 144

Figure 73: Representation of pixel intensity and volume changes of a $15.5 \mu \mathrm{m}$ diameter chondroblast under a high powered optical trap ( $\sim \mathrm{W}$ at sample), with representative pictures at determining time points. The cell membrane becomes 
permeable after $\sim 20$ seconds and the volume increases suddenly. After 35 seconds, the uptake of Trypan blue by the cell becomes visible. 150

Figure 74: The deformation of chondroblasts by the relative movement of 2 optical traps. The dashed circles show the trap locations.

Figure 75: A $19.1 \mu \mathrm{m}$ diameter chondroblast in static suspension (left). Visible cellular deformation of the cell due to an applied fluid shear stress induced by a straight channel flow (right). The circle represents the trap size (approximately $1.6 \mu \mathrm{m}$ in diameter) and location. 156

Figure 76: Average deformation as a function of flow velocity for 64 cells tested. The two data points at 0 velocity are taken before and after the experiment.

Figure 77: Cell displacement as a function of fluid drag for chondroblasts and osteoblasts with 11.4 to $23 \mu \mathrm{m}$ diameters. The modified trap stiffness $\left(k_{d}\right)$ is determined from the slope of Fdrag.d versus $\Delta x$. The maximum variation in modified trap stiffness is $54 \%$. The lines represent the maximum and minimum trap stiffness calculations.

Figure 78: Drag force versus displacement for a $16.5 \mu \mathrm{m}$ diameter chondroblast (P2).

The experiment was repeated with the same cell to assess the variability due to the measurement technique. The trap stiffness was measured to be approximately $1.2 \mathrm{pN} / \mu \mathrm{m}$ with a difference of $3.7 \%$ between the two runs. 161

Figure 79: Modified trap stiffness for chondroblasts with different passages (P1, P2, P3). No significant difference was observed, but we can see a trend of trap stiffness decreasing with increasing de-differentiation

Figure 80: Modified trap stiffness for chondroblasts with different numbers of cilia (N) on their surface. As $\mathrm{N}$ increases, the trap becomes weaker. 164

Figure 81: Modified trap stiffness for chondroblasts tested the same day they were extracted from culture flask, and the following day. 165

Figure 82: Drag force as a function of sphere elongation in the flow direction. $22 \%$ is the maximum deformation seen in our cell experiments.

Figure 83: Modeling of a $10 \mu \mathrm{m}$ radius sphere in a uniform flow (x-direction), placed $50 \mu \mathrm{m}$ from the bottom coverslip. The colors represent the flow velocity in the $x-z$ plane with the scale on the right showing the flow velocity values from 0 (bottom/blue) to $10^{-4} \mu \mathrm{m} / \mathrm{s}$ (top/dark red). 168

Figure 84: Pictures of chondroblasts with the 60x objective. (a) The cell has one small protrusion. (b) Multitude of small protrusions. (c) Very long protrusions affecting the drag force considerably. 
Figure 85: Model of flow velocity around a cell with a single protrusion in (a) the ydirection and (b) the z-direction.

Figure 86: Model of flow velocity around a cell with a six protrusions in all the main axes.

Figure 87: A living, $20 \mu \mathrm{m}$ diameter osteoblastic optically trapped in the microfluidic cross-junction flow. The small white arrows show flowing particles and the doted black arrows show the general flow direction.

Figure 88: Deformation characteristics of a myoblast subjected to the cross junction extensional flow. Low extension rate $\left(<7.8 \mathrm{~s}^{-1}\right)$ and high extension rate $\left(>7.8 \mathrm{~s}^{-1}\right)$ deformation regimes are observed.

Figure 89: The Cartesian coordinate system was established at the center of the fixed cell position and converted to spherical polar coordinates during stress analysis. For all experiments the flow is planar in the $x-y$ plane $\left(\varphi=0^{\circ}\right)$.

Figure 90: (a) Schematics of the uniform flow field around a suspended cell. The plane is zoomed in in (b), where the arrows represent the general flow directions. The maximum normal stress $\left(\sigma_{\max }\right)$ is on the flow axis $\left(0^{\circ}\right.$ and $\left.180^{\circ}\right)$ and the maximum shear stress $\left(\tau_{\max }\right)$ is at $90^{\circ}$ and $270^{\circ}$.

Figure 91: Normalized normal $\left(\sigma_{r}\right)$ and shear stress $\left(\tau_{r \theta}\right)$ behavior as a function of angular position around a sphere in uniform flow, $r / a$ is the dimensionless distance from the center of the sphere.

Figure 92: For a sphere in a uniform flow, normalized shear and normal stresses at the angle of maximum stress as a function of distance from the cell surface. The numbers in italic represent the interrogation areas in pixels for $\mu \mathrm{PIV}$ measurements.

Figure 93: (a) Schematics of the planar extensional flow field around a suspended cell placed at the stagnation point.(b) Magnified view of the light blue plane in (a). The arrows represent the general flow directions. The maximum normal stress $\left(\sigma_{\max }\right)$ is along the inflow and outflow axis and the maximum shear stress $\left(\tau_{\max }\right)$ is offset by $45^{\circ}$. 186

Figure 94: For a planar extensional flow, normalized normal stress without the pressure field $\left(\tau_{r r}\right)$ and shear stress $\left(\tau_{r}\right)$ values as a function of the angle from the horizontal $\theta$ for several values of dimensionless distance from the center of the sphere $(r / a)$.

Figure 95: Normalized shear and normal stresses at the angle of maximum stress as a function of distance from the cell surface. The numbers in italic represent the interrogation areas in pixels for $\mu$ PIV measurements. 


\section{CHAPTER 1}

\section{INTRODUCTION}

Bone and cartilage are complex dynamic systems in constant reorganization throughout life. The cells are embedded in an extra-cellular three-dimensional matrix of fibers and constantly react to their surroundings. Changes in the biochemical composition or osmolarity of the extra cellular matrix and imposed pressures or other forces on the tissue, all compel the cell to trigger a chain of reactions and intracellular biochemical signals. These signals drive the cell to synthesize or resorb bone or cartilaginous tissue as needed. In vivo, this permanent remodeling is necessary to maintain the phenotype and healthy production of surrounding tissue. With respect to bone tissue, the remodeling can be divided into three phases: bone is first subject to resorption, then regrowth, and finally calcification. This constant remodeling allows regeneration of the tissues, the deletion of the small defects that appear with normal activity or injury such as micro-fracture, the reshaping of the bone where needed, and growth after injury. In the first year of life, almost $100 \%$ of the skeleton is replaced. In adults, the rate of remodeling is about $10 \%$ per year. This remodeling, however, is highly influenced by the loads applied to the skeletal system. A skeleton under normal conditions with average activity will undergo a healthy remodeling. However, immobilization causes loss of bone 
and excretion of calcium and phosphorus [Dietrick, 1948]. As seen in Figure 1, long spaceflights where the body is under zero gravity also cause loss of bone [Mack, 1967], [Morey, 1978] if the lack of gravity is not compensated by high activity. The loss of bone weakens the bone structure, increases the risk of fracture, and may lead to osteoporosis which affects 1 in 3 women and 1 in 5 men over the age of fifty [Melton, 1992], [Melton, 1998]. An unbalanced remodeling can also cause other types of diseases such as bone atrophy, hypertrophy, and over-mineralization. When the turnover is too slow, the bone's mechanical properties are altered and the bone becomes brittle. When the turnover is too high, calcification is unfinished and the bone is therefore weak.

The turn-over of cartilaginous tissue is much slower, however the same balance needs to be maintained. It is governed by the amount of cytokines, growth factors and mechanical stimuli imparted on the tissue and more specifically on the chondrocytes (cartilage cells).
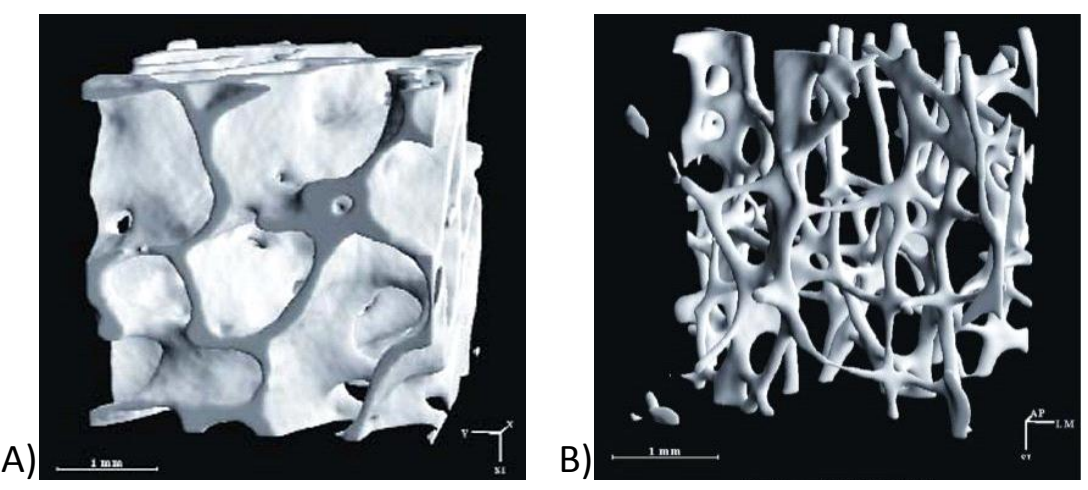

Figure 1: Bone model before and after a long spaceflight (Images from the European Space Agency). 
One of the most common disorders concerning this metabolic balance is osteoarthritis, where the rate of catabolic processes overcomes the rate of cartilage synthesis. This in turn, leads to cartilage loss and degradation of joints [Ehrlich, 1987].

Numerous studies have focused on the cytokines, growth factors, hormones and other molecules responsible for changes in bone and cartilage remodeling in order to determine the cell's response pathway to those triggers. However, it has only been in the last few years that the major focus seeks to understand the sequence of events through which mechanical signals are transduced into intracellular biochemical signals which alter gene expression and modulate cell activities (Figure 2).

Many research groups have applied stress on monolayers of cells in order to understand the overall mechanism of mechanotransduction [Sipe, 2002], a process by which cells convert mechanical stimuli into biochemical activity.

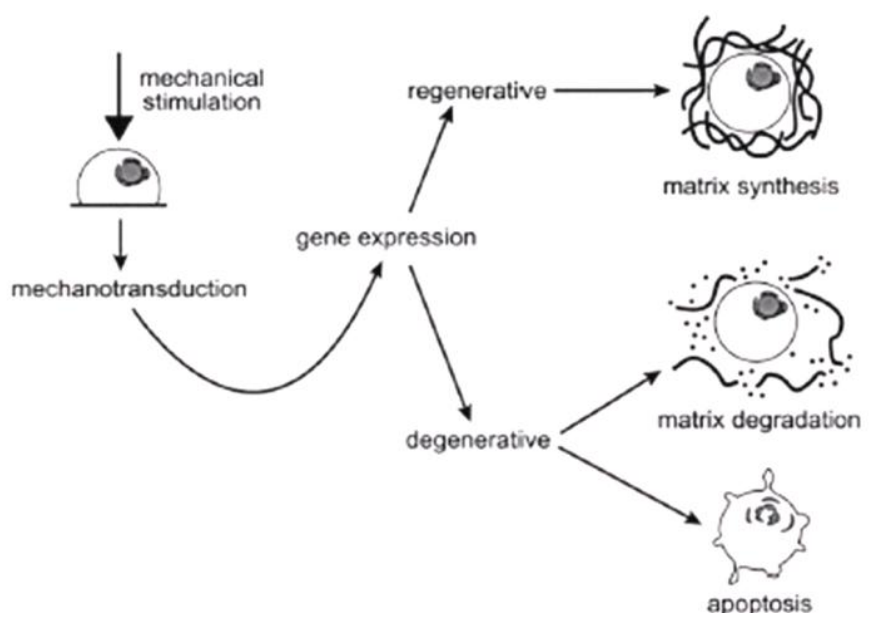

Figure 2: Schematic of cellular response to a mechanical perturbation [Shieh, 2003]. 
Although these studies provide insight into the overall biological effect of mechanical stimuli on cells, the resulting information is always an averaged response over a defined population and does not account for the intercellular heterogeneity such as differences between cells of various cycles, ages, phenotypes, etc. To explore these heterogeneities, single cell biomechanics is required. Recent advances in technology has enabled discovery at the microscale and even the nanoscale. Three major cell techniques, micropipette aspiration, atomic force microscopy, and cytoindentation, allow for the probing of single cells by applying external forces to their membranes. A major disadvantage of these techniques is the required cell attachment to a surface and the localized mechanical stimulus. Cell attachment is known to have an important impact on the cell's response to mechanical stimuli (as developed in the next chapter). However this importance has not been quantified as very few studies (primarily focused on red blood cells) without attachment have been possible. A number of contact-free techniques are available. The oldest contact-free technique is the rheoscope [SchmidSchonbein, 1981] where blood viscosity was tested against red blood cell deformation and aggregation. Later, using the rheoscope as well as an ektacytometer, Bull et al. [Bull, 1983] studied the elliptocytic red cells deformability under different shear stresses. The appearance of optical tweezers and derived technology in the late 1980's opened the door to new ways of testing cells without physical contacts, such as the optical channel [Kaneta, 2001], where red blood cells held in a focused beam were elongated due to 
hydrodynamic stresses. Another optical technique, the optical stretcher [Guck, 2001] holds a cell in place and stretches it. With an unfocused beam, higher powers can be applied without damaging the cell. Interestingly, none of these techniques have been used on bone and cartilage cells.

One of the main objectives of this work is to develop a device capable of studying cellular mechanics and facilitating the characterization of mechanobiology. More specifically, we need a device capable of studying a single cell's mechanical response to a controlled and measurable multiaxial external force without the cell being physically attached to a surface. The $\mu$ PIVOT, a system integrating micron particle image velocimetry ( $\mu$ PIV) and optical tweezers (OT) was developed toward this goal. The laserbased technologies are uniquely custom-integrated to physically hold a cell in the midstream of a fluid flow with OT and monitor the localized fluid velocities and fluidinduced deformations of the cell with $\mu$ PIV. Coupled with microfluidics, the $\mu$ PIVOT allows a sequence of single and multiple axis stresses to be applied to individual cells while measuring the resulting strain response. Studies with the proposed instrument may provide significant insight into the mechanical response of cells, determine the microenvironment most effective in inducing mechanotransduction, contribute to the understanding of pathological cell states and therapeutic approaches for load-bearing tissues, and guide the design of engineered biomaterials which control cellular function. This research represents not only the initial foray into many possible studies on single 
cell biomechanics but also on fluid-interface and fluid-microparticle interactions. The $\mu$ PIVOT enables the simultaneous measurement of the global forces and local stresses applied to a sphere suspended in a fluid flow. The combined information may provide unique insight into theoretical and numerical models of fluid-particle and particleparticle interactions in Newtonian and non-Newtonian fluids. While the scope of potential research with the $\mu$ PIVOT is quite large, this work focuses on the development and validation of the $\mu$ PIVOT instrument and highlights its first applications in research of single cell biomechanics and fluid-particle and particle-particle interactions.

The dissertation is organized as follows: Chapter 2 provides an overview of bone and cartilage cell anatomy, a review of the current status of single cell biomechanics, and a detailed description of the physics behind optical tweezers and micron resolution particle image velocimetry. Chapter 3 describes the $\mu$ PIVOT and its development. Chapter 4 details the $\mu$ PIVOT validation. Chapter 5 examines the dynamics of a single polystyrene sphere suspended in channel, uniform, and extensional flows. Chapter 6 determines the interaction of two trapped microspheres in a uniform flow. Chapter 7 explores the microfluidic and optical tweezers manipulation of biological cells. Chapter 8 provides an analysis of the local fluid stresses and global fluid forces on a suspended sphere in uniform and extensional flows. Finally, Chapter 9 summarizes the current achievements of the $\mu$ PIVOT and discusses its future applications. 
CHAPTER 2

BACKGROUND

\subsection{CELLS, STRESSES AND MECHANOTRANSDUCTION}

\subsubsection{BASICS OF CELL ANATOMY}

Eukaryotic cells are complex fundamental systems forming the basis of all protists, plants, fungi and animals -as opposed to prokaryotic cells (bacteria). The eukaryotic cell is a complex three-dimensional structure with an average diameter of 20 $\mu \mathrm{m}$ that can be modeled by a membrane bounded liquid-matrix combination comprised of organelles with specialized functions. The liquid (cytosol) and the organelles form the cytoplasm, which is enclosed in the cell membrane that functions as a semi-permeable barrier. The cell membrane is a lipid bi-layer that includes many different proteins (active or passive channels, carriers, receptors, etc.) that selectively transport molecules across the membrane while limiting the transport of organically produced chemicals from inside the cell. As depicted in Figure 3, organelles are small, membrane-bound components of the cell of different shapes and sizes. They are numerous and include the nucleus, where most of the DNA is contained, the mitochondria, the endoplasmic reticulum, the Golgi apparatus, ribosomes, peroxisome, lysosomes and so forth. Each have very specific functions. 


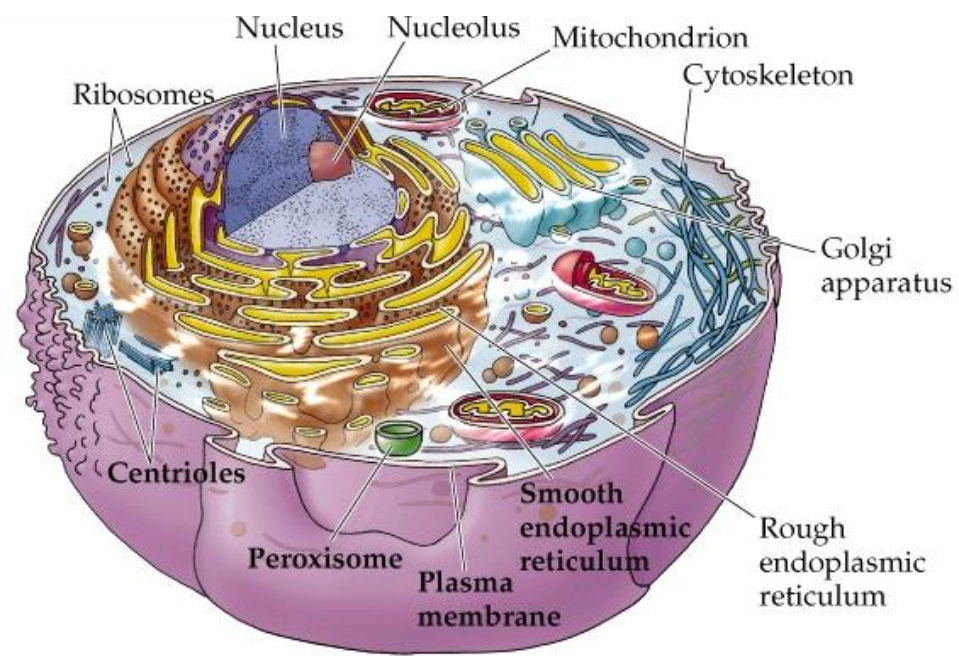

(9) 2001 Sinauer Associates, Inc

Figure 3: Schematic of a eukaryotic cell [http://www.columbia.edu/cu/biology/courses].

Fibrous proteins that form the matrix in the cytoplasm are referred to as the cytoskeleton. These proteins maintain the shape of the cell, anchor organelles, and provide the mechanism for cell movement. There are three kinds of fibers: microtubules, actin filaments, and intermediate filaments (Figure 4). Microtubules are dynamic hollow cylindrical chains of $\alpha$ - and $\beta$-tubulin elements, constantly under polymerization and depolymerization. They function in cell division and serve as a temporary scaffolding for other organelles [Aberts, 1994]. Actin filaments are thin threads that are also dynamic. 


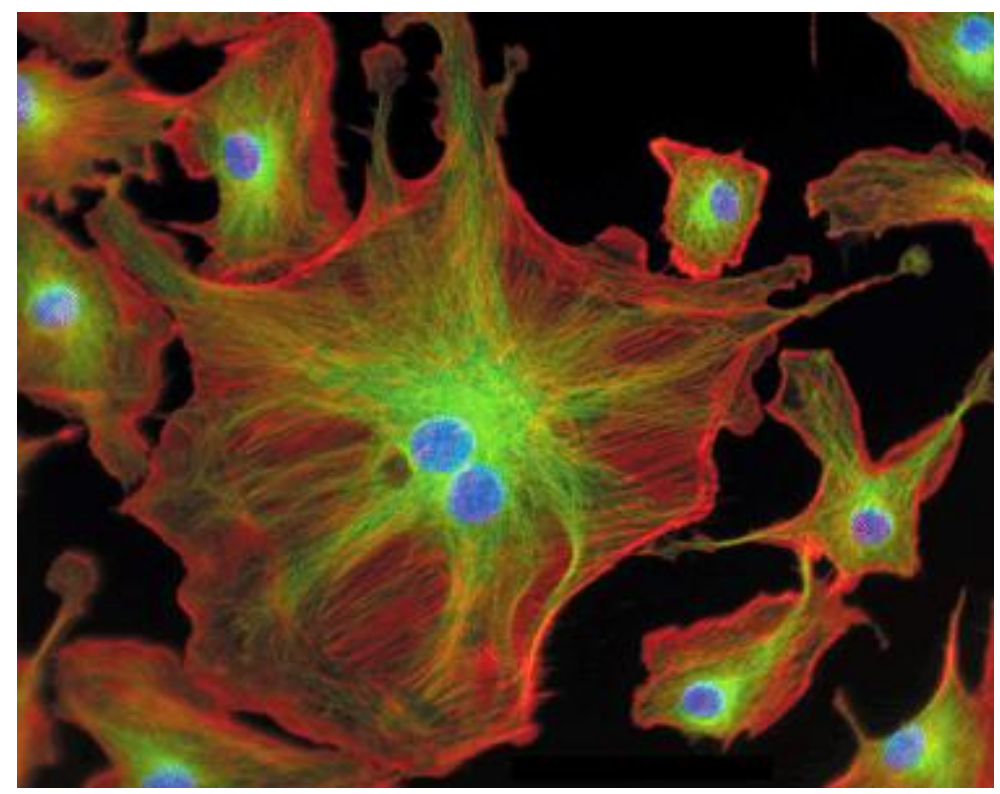

Figure 4: Cytoskeleton of a cow endothelial cell where actin filaments are stained in red and microtubules in yellow. The nucleus is stained in blue (Image from Molecular probes).

They are comprised of polymer chains of the actin protein. They function in cell division, cell motility and adhesion [Aberts, 1994]. They connect the cytoskeleton to the extracellular matrix and transfer the forces between the outside and inside of the cell. Finally, intermediate filaments, as their name suggests, are larger than actin filaments but smaller than microtubules. They are composed of different types of proteins depending on the cell, and their role is not yet well understood. However they seem to transmit force from the periphery of the cell to the nucleus as they are all connected to the nucleus [Aberts, 1994]. Thus microtubules, actin filaments, and intermediate filaments play a key role in ensuring the motility of the cell and mechanical load transmission throughout it. Actin filaments bond to the cell membrane through focal 
adhesions. These structures are complicated macromolecular assemblies containing more than one hundred different proteins [Zamir, 2001]. It has been recently confirmed that focal adhesions concentrate stresses transmitted through the cytoskeleton [Ingber, 1997], [Ingber, 2003] that originate on the cell membrane. Therefore the behavior of the focal adhesion sites and thus the behavior of the entire cell is different depending on the cell's attachment to a substrate or to the extra cellular matrix.

Although most cells are structured as described above, they differ tremendously depending on their location and function. The biological aspects of this research focus on bone and cartilage cells.

\subsubsection{Bone cells}

Bone tissue is a specialized form of connective tissue and is the main element of the skeletal tissues. It is composed of cells and an extracellular matrix in which fibers are embedded. Bone tissue is the only connective tissue where the extracellular matrix becomes calcified. The functions of bone tissue are not only to provide the internal support of the body (with connections to tendons and muscles), but also to provide protection for the vital organs of the body and the hematopoietic bone marrow (blood production) and storage of calcium and phosphate. Bone is a hard and brittle tissue that is dynamic. Throughout life bone tissue is continually being formed and resorbed. By its shape and composition, it can be divided into two categories: spongy (also called trabecular bone or cancellous bone) and compact (also called cortical bone). As seen in 
Figure 5, the hard outer layer is composed of compact bone tissue, with a tight organization of fibers, cells and canals. This tissue accounts for $80 \%$ of the total bone mass of an adult skeleton. Filling the interior of the space is the spongy bone tissue which is composed of a network of branching bone trabeculae allowing room for blood vessels and marrow. Trabecular bone accounts for the remaining $20 \%$ of total bone mass, but has nearly ten times the surface area of compact bone. There are several types of cells within bone: osteoblasts, osteocytes and osteoclasts. Osteoblasts are immature bone cells. They are bone-forming cells that are located on the surface of bone and synthesize a protein mixture (mostly composed of Type I collagen) known as osteoid, which mineralizes to become bone. Osteocytes are mature bone cells. They originate from osteoblasts which have migrated into bone matrix which they themselves produce. The spaces which they occupy are known as lacunae.

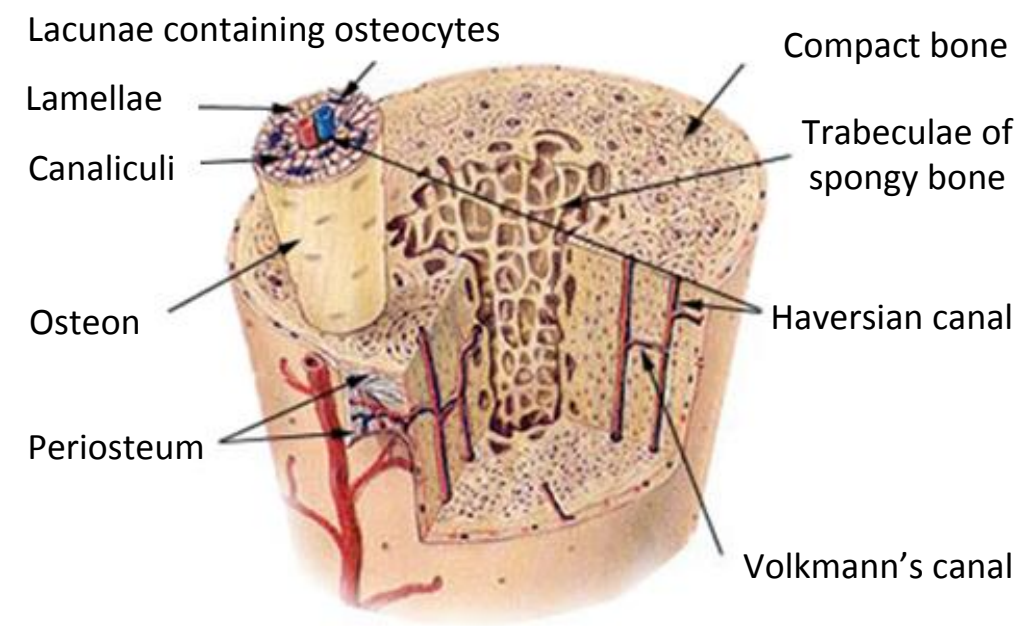

Figure 5: Anatomy of bone (www.web-books.com). 
Osteocytes have many cytoplasmic extensions which reach out to meet other osteocytes in canaliculi. Their functions include to varying degrees: formation of bone, matrix maintenance and calcium homeostasis. They have also been shown to act as mechano-sensory receptors - regulating the bone's response to stress and mechanical load. Lastly, osteoclasts are the cells responsible for bone resorption. Osteoclasts are large, multinucleated cells located on the eroded surface of the bone, equipped with phagocytic-like mechanisms similar to macrophages. For healthy tissues, these three types of bone cells communicate with each other to create a strong and healthy bone structure that is constantly remodeling depending on the needs dictated by the mechanical and chemical environment.

\subsubsection{Cartilage tissue}

Cartilage is another type of dense connective tissue. It contains chondrocytes that produce a large amount of extracellular matrix composed of collagen fibers, elastin fibers, and an abundant ground substance rich in proteoglycan. Depending on the relative amounts of these three main components, cartilage is classified as elastic cartilage, hyaline cartilage, or fibrocartilage. A unique aspect of cartilage is its absence of blood vessels or nerves. Chondrocytes are fed by diffusion enhanced by the compression or flexion of the entire tissue. Thus, compared to other connective tissues, cartilage grows and repairs more slowly. However, the chondrocyte is an easy cell to study, as it needs very low concentrations of oxygen and can live in a harsh environment 
for extended periods of time. As seen in Figure 6, the chondrocytes are located in fluidfilled lacunae spread far apart among the extracellular matrix mainly composed of collagenous fibers. With its smooth surface, articular cartilage's primary function is to reduce the friction in joints. It is extremely strong but very flexible and elastic. As seen in Figure 6, it is composed of five different zones depending on the orientation of the collagen fibers: the superficial zone that is resistant to shear due to its tangential arrangement; the transitional zone that is resistant to compression; the radial zone that is resistant to compression with its columnar arrangement; the tidemark that is resistant to shear; and finally the calcified zone that acts as an anchor between articular cartilage and subchondral bone.

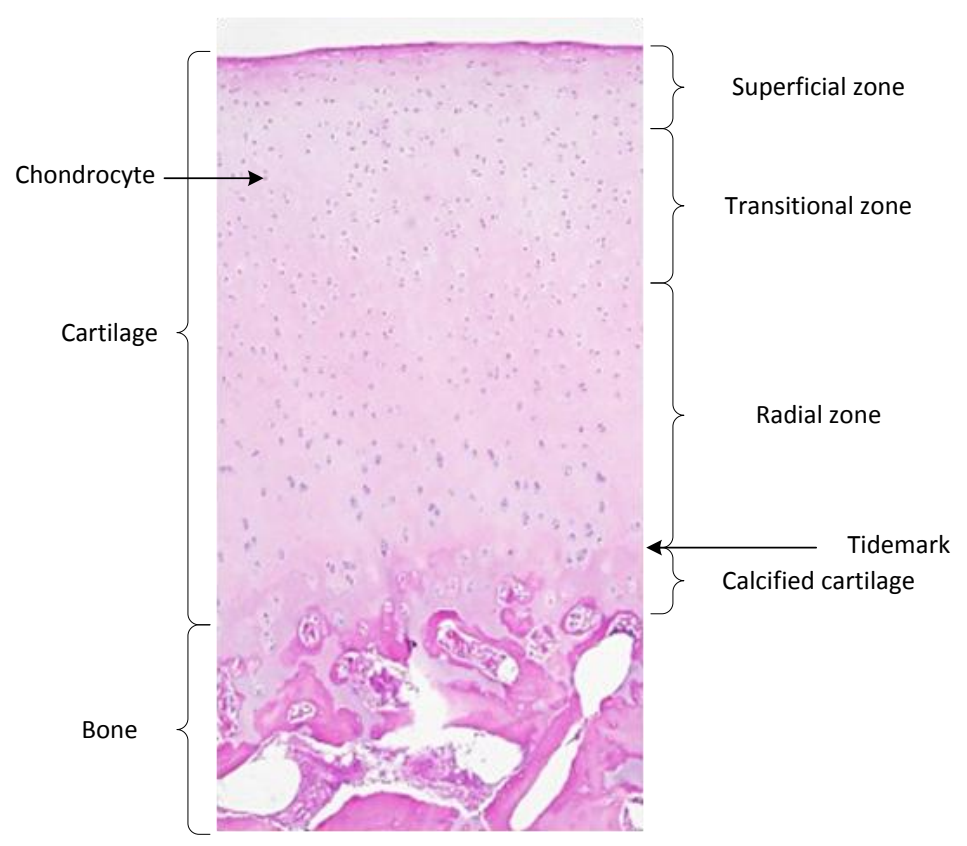

Figure 6: Picture of articular cartilage stained with Hematoxylin and Eosin (image from the University of Western Australia- School of Anatomy and Human Biology). 
These zones are clearly visible due to the difference in extracellular matrix. However, the chondrocytes of each zone do not exhibit obvious differences in their morphology or physiology.

\subsubsection{MECHANOTRANSUCTION}

Huang et al. [Huang, 2004] provide a review of mechanotransduction, more specifically how externally applied forces are transmitted into and throughout the cell, as well as the magnitudes and distribution of force corresponding to these different methods of stimulation. They show that the strain field is widely distributed throughout the cell in a non-homogenous matter and tends to be concentrated at regions around focal adhesions [Ingber, 2003]. The levels of force needed to elicit a response by fluid shear are approximately $1 \mathrm{~Pa}$. Hu et al. [Hu, 2003] used magnetic twisting cytometry to observe local strains by measuring the displacement of labeled mitochondria. They estimated local stresses to be in the range of several hundred pascals, translated to tens of piconewtons exerted on single molecules if considering that those stresses are condensed at the focal adhesion sites. Aside from the amplitude of the stress, its frequency has been reported to be of primary importance. Cyclic or dynamic loading has been shown to enhance extra cellular matrix synthesis activity compared to static loading, with only a short time period of loading necessary to initiate an adaptive response. The frequency of $1 \mathrm{~Hz}$, which is the typical physiological frequency (heart frequency, average frequency of the human walk) seems to be optimum. Jin et al. [Jin, 
2000] found that excessive shear stress stimulation of chondrocytes promoted a transcription regulator, matrix metalloproteinase- 9 , as a mediator of the progressive degradation leading to Osteoarthritis. Despite these findings, the mechanisms through which bone and cartilage cells perceive and transduce extracellular mechanical signals are not well understood.

\subsection{SINGLE CELL BIOMECHANICS}

Exploration of mechanotransduction relies on the use of different methods to apply mechanical forces to living cells. One of the central achievements in mechanotransduction has been the development of carefully designed devices to impose mechanical forces. Two kinds of experiments prevail: First, those aimed to obtain information from a group of thousands of cells that can then be averaged and estimated for one cell. The predominant studies have been conducted using membrane stretching, shear stress, and hydrostatic pressures. They have shown that large groups of cells respond to mechanical stimuli by increasing their intracellular calcium concentration, a wide variety of signaling molecules, and different proteins.

The need of exploring the mechanisms of mechanotransduction at a smaller level, the cellular level, is now becoming critical [Shieh, 2002]. The monolayer studies provide an average response of the cell, which can be significantly different from what a single cell experiences. Additionally, the force applied to the monolayer is a bulk force and doesn't elicit the response imposed on a cell with a specific morphology and 
composition due to local mechanical stimuli. With advances in technology, analyzing single cell reaction is becoming feasible.

Within the past two decades, several methods have been developed to directly determine the mechanical properties of individual cells. The most common (described further later) are micropipette aspiration, cytoindentation, and atomic force microscopy. Although none of them can realistically replicate the in-vivo environment, they provide invaluable information on the level and type of mechanical stimulus needed to induce cellular activity or change in the cell physical characteristics. For each of the methods, the cell is mechanically provoked and its response is recorded. Combined with simplified mathematical models, mechanical cell properties are inferred from the resulting information.

\subsubsection{MATHEMATICAL MODELS}

The simplest model is the Law of Laplace model where the cell is a fluid drop enclosed in a shell. As shown in the work of Hochmuth [Hochmuth, 2000] where cells were manipulated with micropipette aspiration, this model yields the surface tension property as well as the cell elastic modulus. A slightly more elaborate model is the punch model. It treats the cell as a linearly elastic, homogeneous, isotropic, incompressible and semi-infinite solid. Theret et al.[Theret, 1988] has applied this model to cells tested with micropipette aspiration. They obtained Young's modulus for bovine endothelial cells undergoing different shear stresses. Note: As a reminder, the Young's 
modulus $(E)$ is by definition the modulus of elasticity for an isotropic elastic material. In this model, it can indeed be calculated with a simple equation when knowing the force applied on the cell, the Poisson's ratio of the cell, the geometric properties of the instrument probing the cell and the cell deformation. In increasing complexity, the viscoelastic model accounts for the time-dependency of the cell's mechanical response. The most common viscoelastic model is the standard linear solid (SLS), where the cell is represented as a circuit of springs and dashpots with the cell being part viscous fluid and part elastic solid [Ozkaya, c1999]. Because the cell is not considered as an isotropic elastic material, parameters other than the Young's modulus are used to define the material. They are the instantaneous modulus $\left(E_{0}\right)$, the relaxed modulus $\left(E_{\infty}\right)$, and the apparent viscosity $(\mu)$ [Koay, 2003]. Finally, the most sophisticated widely used model is the linear biphasic model. The cell is modeled as a porous solid medium saturated with fluid. To solve the model equations, it is generally assumed that the solid medium is linearly elastic, isotropic and incompressible, while the fluid is inviscid and incompressible [Mow, 1980]. The properties retrieved from this model are the aggregate modulus, the Poisson's ratio of the solid phase, and the permeability.

Each of these models have been applied to the single cell experimental results of micropipette aspiration, cytoindentation, and atomic force microscopy to determine the mechanical properties of the cell. 


\subsubsection{SINGLE CELL TECHNIQUES}

\subsubsection{Micropipette aspiration}

Micropipette aspiration is a pioneering technique that has been applied to numerous cell types. It applies a negative pressure (subatmospheric) to partially aspirate the cell. The difference in pressure produces a localized membranous stretching (Figure 7). Previous studies have used the micropipette aspiration on various cell types, starting with leucocytes [Schmid-Schonbein, 1981]. Hochmuth et al. [Hochmuth, 1987] measured the red blood cell membrane elasticity and viscosity, Evans et al. [Evans, 1989] measured the apparent viscosity and cortical tension of blood granulocytes, Sato et al. [Sato, 1987] studied the mechanical properties of bovine endothelial cells subject to shear stress.

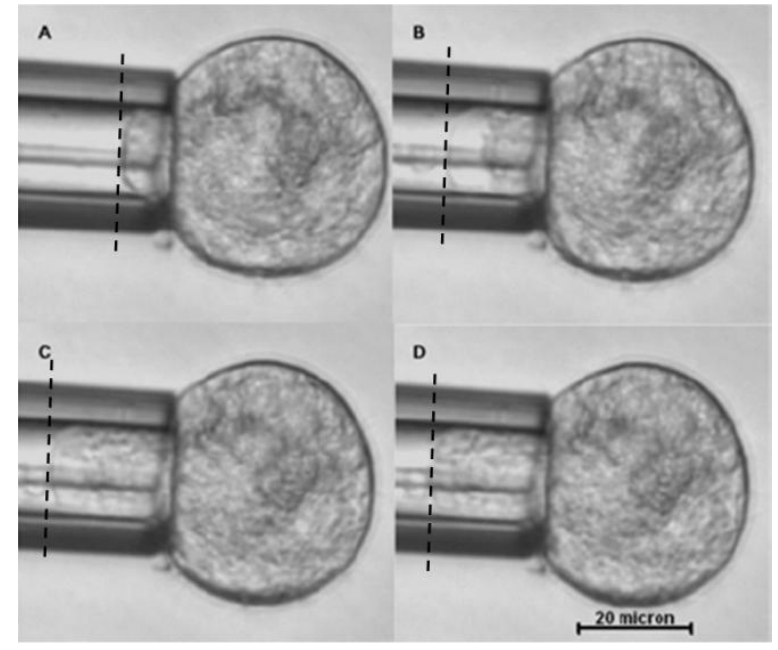

Figure 7: Micropipette aspiration of hMSCs at room temperature. Images (A-D) are displayed at time $t=$ $1 \mathrm{~s}, 15 \mathrm{~s}, 100 \mathrm{~s}$ and $200 \mathrm{~s}$ after the application of step aspiration pressure, respectively [Tan, 2008]. 
Regarding chondrocytes, Jones et al. [Jones, 1999] compared healthy chondrocytes to those originating from osteoarthritic cartilage. They applied maximum pressures between 0.05 and $1 \mathrm{kPa}$. Using the punch model, they determined a Young's modulus of $0.65 \mathrm{kPa}$ and $0.67 \mathrm{kPa}$ for the healthy and diseased cells respectively (no significant difference). However they observed a significant difference in volume change, with a higher deformation for the osteoarthritic chondrocytes. More recently, Zhang et al. [Zhang, 2008] also compared healthy versus osteoarthritic chondrocytes. Testing the cells with micropipette aspiration and using a viscoelastic model, they reported a significantly lower equilibrium modulus E (0.39 kPa versus $0.55 \mathrm{kPa})$, instantaneous modulus $\mathrm{E}_{0}(0.68 \mathrm{kPa}$ versus $0.98 \mathrm{kPa})$ and apparent viscosity $\mu(0.39 \mathrm{kPa} . \mathrm{s}$ versus 6.36 kPa.s) for the diseased cells.

\subsubsection{Cytoindentation}

Cytoindentation compresses a cell adhered to a surface, using a $5 \mu \mathrm{m}$ diameter glass microfiber probe. Petersen et al. [Petersen, 1982] probed a mouse fibroblast and explored the dependence of the cell deformability with temperature, location of the perturbation, and cytochalasin B. Pasternak et al. [Pasternak, 1985] measured the deformability of lymphocytes triggered by cross-linking surface receptors. Felder et al. [Felder, 1990] analyzed the forces and motions at the leading lamellas of fibroblasts. More recently, Shin et al. [Shin, 1999] used cytoindentation to study MG63 osteosarcoma cells. According to Ofek et al. [Ofek, 2007] this was the earliest model of 
the cytoindenter. In their results, the cells had a permeability $\mathrm{k}$ of $1.18 \times 10^{-10} \mathrm{~m}^{4} \mathrm{~N}^{-1} \mathrm{~s}^{-1}$, an aggregate modulus of $2.05 \mathrm{kPa}$, shear modulus of $0.41 \mathrm{kPa}$, and Poisson's ratio of 0.37. Finally, Koay et al. [Koay, 2003] used the cytoindenter to apply creep tests on single chondrocytes at a test load of $50 \mathrm{nN}$ for either 15 or 20 seconds. They used two mathematical models: the punch and the viscoelastic models. The punch model yielded an average Young's modulus of $1.10 \mathrm{kPa}$. The viscoelastic model resulted in an instantaneous modulus of $8 \mathrm{kPa}$, relaxed modulus of $1.01 \mathrm{kPa}$, and apparent viscosity of $1.5 \mathrm{kPa} . \mathrm{s}$ for the chondrocyte.

\subsubsection{Atomic Force Microscopy (AFM)}

AFM was first invented in 1986 for high precision topographic images of hydrated material surfaces such as those of cells [Binnig, 1986]. However it has been used extensively since then to apply mechanical stimuli on cells because of its highresolution scanning and nanoindentation capabilities. As seen in Figure 8, the principle is relatively simple: a laser is reflected off a cantilever probe (tip in the order of nanometers to tenths of micrometers) and is tracked on a photodetector. When the probe interacts with the cell, it deflects with the deflection recorded using the laser position detector. Knowing the probe spring constant and the geometry of the tip, one can obtain information on the cell mechanical properties from these results. 


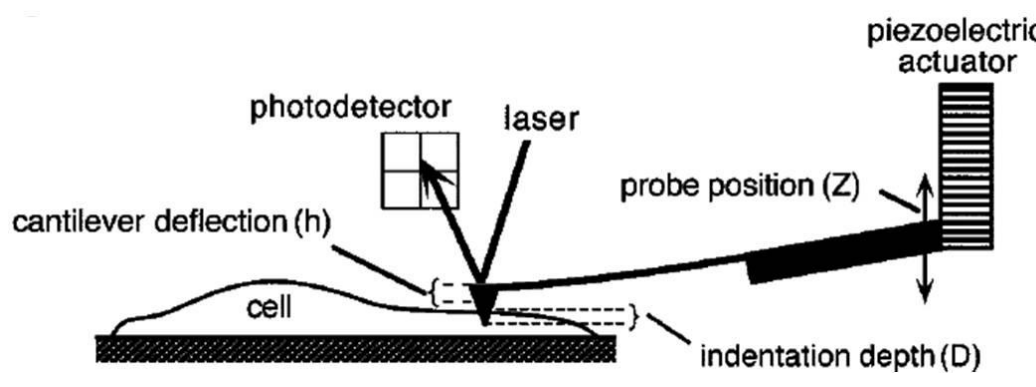

Figure 8: Schematic of AFM cell indentation experiment [Costa, 2006].

Hassan et al. [Hassan, 1998] used AFM for a microeslastic mapping of epithelial cells.

Rotsch et al. [Rotsch, 2000] reported the changes of cytoskeletal structure and mechanics of fibroblasts when subjected to different types of drugs. Regarding chondrocytes or osteoblasts, Darling et al.[Darling, 2006] examined porcine articular chondrocytes from different zones using AFM. Applying a force of $2.5 \mathrm{nN}$ on the center of the cell for 60 seconds, the results show that superficial zone chondrocytes were stiffer than middle/deep cells in terms of instantaneous modulus $(0.55 \mathrm{kPa}$ versus 0.29 $\mathrm{kPa})$ and relaxed modulus ( $0.31 \mathrm{kPa}$ versus $0.17 \mathrm{kPa})$, and had a greater apparent viscosity (1.15 kPa.s versus $0.61 \mathrm{kPa} . \mathrm{s})$. In this study, they also compared these conclusions to results obtained on middle/deep cells using the micropipette aspiration technique. They had similar cell moduli, but there was a higher apparent viscosity for the measurements from the latter technique. More recently, Chaudhuri et al., [Chaudhuri, 2009] combined AFM with side-view optical imaging to obtain fluorescent images of the deformed cell (with views of the deformation along the loading axis) while controlling the force imparted on it. 


\subsubsection{Main results for single chondrocytes}

Below are the results from the main publications on single cell experiments conducted on chondrocytes. This table combines the results published in Ofek and

Athanasiou's review [Ofek, 2007] as well as the results from newer publications.

\begin{tabular}{|c|c|c|c|c|}
\hline $\begin{array}{l}\text { Mathematical } \\
\text { model }\end{array}$ & $\begin{array}{l}\text { Experimental } \\
\text { technique }\end{array}$ & Tissue source & Material properties & Ref \\
\hline Punch & Micropipette & $\begin{array}{l}\text { Healthy human articular } \\
\text { cartilage }\end{array}$ & $E_{Y}=0.65 \mathrm{kPa}$ & $\mathrm{E}$ \\
\hline Elastic & Cytoindentation & Bovine metatarsal joints & $\mathrm{E}_{\mathrm{Y}}=1.1 \mathrm{kPa}$ & $\mathrm{F}$ \\
\hline Elastic & $\begin{array}{l}\text { Unconfined } \\
\text { compression }\end{array}$ & Bovine metatarsal joints & $E_{Y}=2.55 \mathrm{kPa}$ & G \\
\hline Punch & Micropipette & $\begin{array}{l}\text { Canine femoral articular } \\
\text { cartilage, } \\
\text { superficial zone }\end{array}$ & $E_{Y}=24 \mathrm{kPa}$ & $\mathrm{D}$ \\
\hline Punch & Micropipette & $\begin{array}{l}\text { Canine femoral articular } \\
\text { cartilage }\end{array}$ & $E_{Y}=23.2 \mathrm{kPa}$ & $\mathrm{D}$ \\
\hline Punch & Micropipette & $\begin{array}{l}\text { Healthy human articular } \\
\text { cartilage, } \\
\text { superficial zone }\end{array}$ & $E_{Y}=68.9 \mathrm{kPa}$ & A \\
\hline Punch & Micropipette & $\begin{array}{l}\text { Healthy human articular } \\
\text { cartilage }\end{array}$ & $E_{Y}=62.0 \mathrm{kPa}$ & A \\
\hline \multirow{3}{*}{ Viscoelastic } & \multirow{3}{*}{ AFM } & \multirow{3}{*}{$\begin{array}{l}\text { Porcine femoral joints, } \\
\text { superficial zone }\end{array}$} & $\mathrm{E}_{0}=0.55 \mathrm{kPa}$ & \multirow{3}{*}{ C } \\
\hline & & & $E_{\infty}=0.31 \mathrm{kPa}$ & \\
\hline & & & $\mu=1.15 \mathrm{kPa} . \mathrm{s}$ & \\
\hline \multirow{3}{*}{ Viscoelastic } & \multirow{3}{*}{ AFM } & \multirow{3}{*}{ Porcine femoral joints } & $\mathrm{E}_{0}=0.29 \mathrm{kPa}$ & \multirow{3}{*}{$\mathrm{C}$} \\
\hline & & & $E_{\infty}=0.17 \mathrm{kPa}$ & \\
\hline & & & $\mu=0.41 \mathrm{kPa} . \mathrm{s}$ & \\
\hline \multirow{3}{*}{ Viscoelastic } & \multirow{3}{*}{ Micropipette } & \multirow{3}{*}{ Porcine femoral joints } & $\mathrm{E}_{0}=0.45 \mathrm{kPa}$ & \multirow{3}{*}{ C } \\
\hline & & & $\mathrm{E}_{\infty}=0.14 \mathrm{kPa}$ & \\
\hline & & & $\mu=2.57 \mathrm{kPa} . \mathrm{s}$ & \\
\hline \multirow{3}{*}{ Viscoelastic } & \multirow{3}{*}{ Micropipette } & \multirow{3}{*}{$\begin{array}{l}\text { Healthy human articular } \\
\text { cartilage }\end{array}$} & $\mathrm{E}_{0}=0.41 \mathrm{kPa}$ & \multirow{3}{*}{ I } \\
\hline & & & $\mathrm{E}_{\infty}=0.24 \mathrm{kPa}$ & \\
\hline & & & $\mu=3.0 \mathrm{kPa} . \mathrm{s}$ & \\
\hline \multirow{3}{*}{ Viscoelastic } & \multirow{3}{*}{ Cytoindentation } & \multirow{3}{*}{ Bovine metatarsal joints } & $\mathrm{E}_{0}=8.0 \mathrm{kPa}$ & \multirow{3}{*}{$\mathrm{F}$} \\
\hline & & & $\mathrm{E}_{\infty}=1.09 \mathrm{kPa}$ & \\
\hline & & & $\mu=1.50 \mathrm{kPa} . \mathrm{s}$ & \\
\hline \multirow{2}{*}{ Viscoelastic } & \multirow{2}{*}{$\begin{array}{l}\text { Unconfined } \\
\text { compression }\end{array}$} & \multirow{2}{*}{ Bovine metatarsal joints } & $\mathrm{E}_{0}=2.47 \mathrm{kPa}$ & \multirow{2}{*}{ G } \\
\hline & & & $\mathrm{E}_{\infty}=1.48 \mathrm{kPa}$ & \\
\hline
\end{tabular}




\begin{tabular}{|c|c|c|c|c|}
\hline & & & $\mu=1.92 \mathrm{kPa} . \mathrm{s}$ & \\
\hline \multirow{3}{*}{ Viscoelastic } & \multirow{3}{*}{$\begin{array}{l}\text { Unconfined } \\
\text { compression }\end{array}$} & \multirow{3}{*}{$\begin{array}{l}\text { Bovine metatarsal joints, } \\
\text { superficial zone }\end{array}$} & $\mathrm{E}_{0}=1.59 \mathrm{kPa}$ & \multirow{3}{*}{$\mathrm{H}$} \\
\hline & & & $\mathrm{E}_{\infty}=1.20 \mathrm{kPa}$ & \\
\hline & & & $\mu=6.32 \mathrm{kPa} . \mathrm{s}$ & \\
\hline \multirow{3}{*}{ Viscoelastic } & \multirow{3}{*}{$\begin{array}{l}\text { Unconfined } \\
\text { compression }\end{array}$} & \multirow{3}{*}{ Bovine metatarsal joints } & $\mathrm{E}_{0}=0.69 \mathrm{kPa}$ & \multirow{3}{*}{$\mathrm{H}$} \\
\hline & & & $\mathrm{E}_{\infty}=0.49 \mathrm{kPa}$ & \\
\hline & & & $\mu=3.18 \mathrm{kPa} . \mathrm{s}$ & \\
\hline \multirow{3}{*}{ Viscoelastic } & \multirow{3}{*}{ Micropipette } & & $\mathrm{E}_{0}=0.98 \mathrm{kPa}$ & \multirow{3}{*}{$J$} \\
\hline & & & $\mathrm{E}_{\infty}=0.55 \mathrm{kPa}$ & \\
\hline & & & $\mu=6.36 \mathrm{kPa} . \mathrm{s}$ & \\
\hline \multirow{3}{*}{ Biphasic } & \multirow{3}{*}{$\begin{array}{l}\text { Unconfined } \\
\text { compression }\end{array}$} & \multirow{3}{*}{ Bovine metatarsal joints } & $\mathrm{H}_{\mathrm{A}}=2.58 \mathrm{kPa}$ & \multirow{3}{*}{ G } \\
\hline & & & $\mathrm{k}=2.57 \times 10^{-12} \mathrm{~m}^{4} \mathrm{~N}^{-1} \mathrm{~s}^{-1}$ & \\
\hline & & & $v_{s}=0.069$ & \\
\hline Biphasic & Micropipette & $\begin{array}{l}\text { Healthy human articular } \\
\text { cartilage }\end{array}$ & $v_{s}=0.38$ & I \\
\hline \multirow[b]{2}{*}{ Multiscale biphasic } & \multirow[b]{2}{*}{ Micropipette } & \multirow{2}{*}{$\begin{array}{l}\text { Healthy human articular } \\
\text { cartilage, } \\
\text { superficial zone }\end{array}$} & $E_{Y}=39.7 \mathrm{kPa}$ & \multirow[b]{2}{*}{$\mathrm{B}$} \\
\hline & & & $\mathrm{k}=4.71 \times 10^{-17} \mathrm{~m}^{4} \mathrm{~N}^{-1} \mathrm{~s}^{-1}$ & \\
\hline \multirow{2}{*}{ Multiscale biphasic } & \multirow{2}{*}{$\begin{array}{l}\text { Micropipette } \\
\text { Aspiration }\end{array}$} & \multirow{2}{*}{$\begin{array}{l}\text { Healthy human articular } \\
\text { cartilage }\end{array}$} & $E_{Y}=36.8 \mathrm{kPa}$ & \multirow{2}{*}{ B } \\
\hline & & & $\mathrm{k}=3.69 \times 10^{-17} \mathrm{~m}^{4} \mathrm{~N}^{-1} \mathrm{~s}^{-1}$ & \\
\hline
\end{tabular}

Table 1: List of mathematical models used for different experimental techniques with the resulting cell material properties. The right column indicates the references from which the data was taken: A: [Alexopoulos, 2003], B: [Alexopoulos, 2005], C: [Darling, 2006], D: [Guilak, 2005], E: [Jones, 1999], F: [Koay, 2003], G: [Leipzig, 2005], H: [Shieh, 2006], I: [Trickey, 2000], J: [Zhang, 2008].

Compressive stresses of 1 to $10 \mathrm{MPa}$ are experienced by cartilage in hip joints when simply walking. However at the cell level, studies have shown that forces as little as a few piconewtons initiate $\left[\mathrm{Ca}^{2+}\right]$ signaling. This response is known to be one of the first reactions of cells to mechanical stimuli. However, as shown above, only forces of a few nanonewtons are necessary to assess the mechanical properties of the cells. From the results summarized in the table, the single cells modulus of elasticity $\left(E_{Y}\right)$ of chondrocytes is on the order of a few $\mathrm{kPa}$. The relaxed $\left(E_{\infty}\right)$ and the instantaneous $\left(E_{0}\right)$ moduli seem to be on the same order of magnitude but slightly lower than $E_{Y}$. 
Surprisingly, these results can vary up to two orders of magnitude depending on the technique and the mathematical model used.

The above single cell studies provide insight into the response of cells to mechanical stimuli allowing exploration of its mechanical properties. However, the cell is perturbed at a single spot and has a wide contact area with the testing material. Thus, the stimulus remains very localized and specific. Additionally, with a relatively long experimental set up time, only a relatively few cells can be realistically tested under similar conditions. A goal of this work is to apply mechanical stimuli in a global fashion (contrary to localized) without physical attachment. Optical tweezing provides a method for manipulating cells without physical attachments.

\section{$2.3 \quad$ OPTICAL TWEEZERS}

Optical tweezers or optical trapping (OT) is a laser based technique capable of suspending and manipulating micron-sized objects with nanometer position detection and applied forces on the order of pico-Newtons [Ashkin, 1987], [Lang, 2002]. For a detailed review of optical tweezers, see [Svoboda, 1994], and [Neuman, 2004]. In brief, an optical trap is produced by passing a laser beam through a high numerical aperture objective lens and focusing it to its diffraction-limited spot. Traditionally there are two main theories that explain the trapping mechanisms in optical tweezers. For particles much smaller in dimension than the wavelength of the trapping laser, trapping can be explained by treating the object as an induced point dipole that is affected by an 
electromagnetic field, the laser beam. The trapping force arises from the interaction of the induced dipole with the gradient of the field, with the highest intensity being in the center of the beam (Gaussian beam). This theory, using wave optics, is referred to as the Rayleigh regime. For particles larger in dimension than the wavelength of the trapping laser (Mie regime), a ray optics approach indicates that individual rays of light are refracted as they pass through the trapped object. This change in direction and the associated momentum imparts an equal and opposite force on the object. Without additional imposed forces, an object is trapped at the focal point [Ashkin, 1992]. This theory, outlined in more detail below, is applicable to the experiments of this work as the particles (spheres or cells) are at least ten times larger than the laser wavelength. For a trapped particle with dimensions on the same order of magnitude as the laser's wavelength, more complex electromagnetic theories need to be assessed, although it is this dimension where the trap is the most effective [Svoboda, 1994]. Regardless of theoretical trapping regime, actual trap behavior and trapping force are generally calibrated experimentally as the trapping force depends on the particle type, laser power, particle shape, particle size, and fluid media.

\subsubsection{THEORY OF OPTICAL TWEEZERS IN THE MIE REGIME}

Figure 9 provides an illustration of the trapping forces in the Mie regime. Taking a typical pair of rays $a$ and $b$ passing through the objective lens and converging at the focus point $f$, the refraction of those rays when going through a dielectric sphere gives 
rise to forces $\overrightarrow{F_{a}}$ and $\overrightarrow{F_{b}}$ respectively imparted on the sphere. As shown in the schematics of Figure 9 with the sphere being either above (A), below (B), or displaced horizontally (C) from $\mathrm{f}$, the vector sum $\overrightarrow{F_{T}}$ is always pointing towards the trap focus. For an arbitrary position near the trap focus, the dielectric sphere is always attracted towards that focus and becomes "trapped by light". This surprising result was discovered empirically by Ashkin in 1986 (Bell's Laboratories) and was quantified a few years later [Ashkin, 1992].

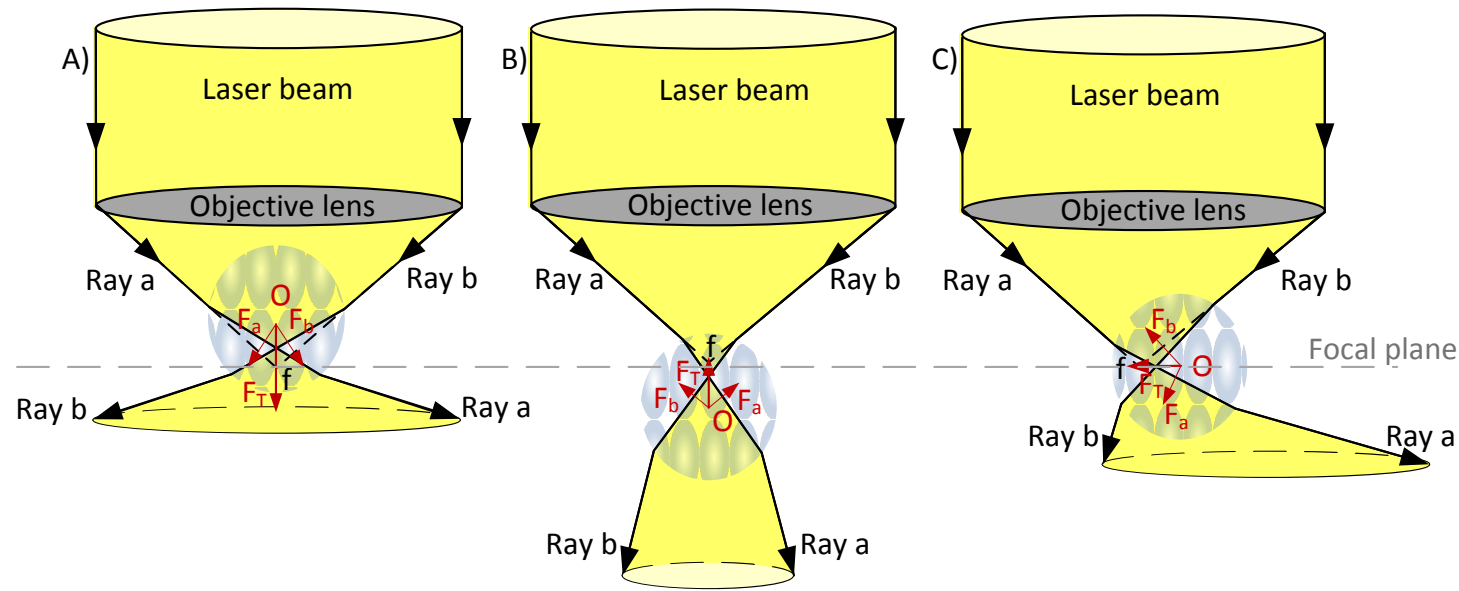

Figure 9: Qualitative view of the trapping of a dielectric sphere.

\subsubsection{Forces imparted to a sphere due to a single ray}

In order to understand and quantify the forces due to the optical trap, let's first consider a single ray of light of power $\mathrm{P}$ propagating in a medium of index of refraction $n_{1}$. The amplitude of this ray's momentum per second is equal to $M_{P}=n_{1} P / c$, with $c$ being the speed of light. As shown in Figure 10, when coming across an object of index 
of refraction $n_{2}\left(n_{1} \neq n_{2}\right)$ this ray is divided into two components at the interface: one part of the ray is reflected (power PR, with $R$, the Fresnel Reflection coefficient) and the other part of the ray is transmitted (power PT, with T, the Fresnel Transmission coefficient) into the object. The conservation of energy is maintained with $R+T=1$. The angles that the incident and transmitted rays make to the normal of the interface are given as $\theta$ and $\sigma$ respectively. The relationship between the two angles is given by Snells' law, $\mathrm{n}_{1} \cdot \sin \theta=\mathrm{n}_{2} \cdot \sin \sigma$ (This relationship is valid only in specular media).

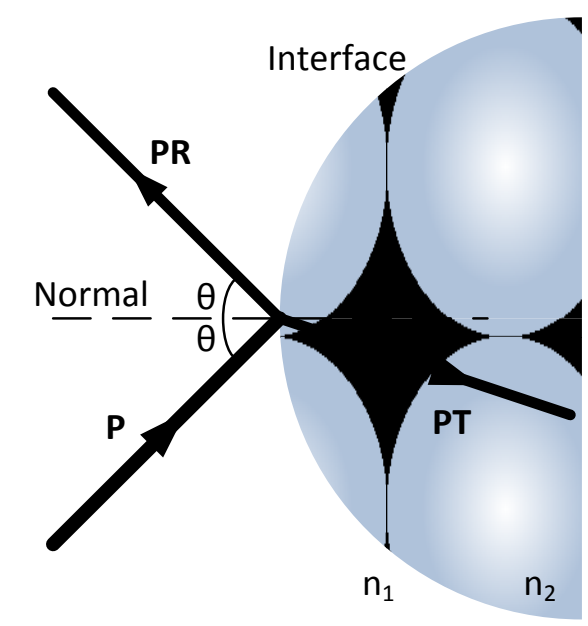

Figure 10: Schematics of Snell's law.

For an object of perfect spherical shape, the force imparted on the sphere by the ray of light has an exact solution developed by Ashkin [Ashkin, 1992]. The theory is as follows. Figure 11 shows when the ray of Power P hits the sphere, it partially refracts, giving rise to a series of scattered rays exiting the sphere with the powers $P R, P T^{2}, P^{2} R$, 
$\ldots, P T^{2} R^{n} . \ldots .$, and flux of momentum $\overrightarrow{M_{P R}}, \overrightarrow{M_{P T^{2}}}, \overrightarrow{M_{P T^{2} R}}, \ldots, \overrightarrow{M_{P T^{2} R^{n}}}$ respectively, with, for each refracted ray, the direction of the momentum being the same as the ray, and the amplitude equal to the multiplication of the power by $n_{1} / c$. These scattered rays make angles relative to the incident ray direction of $\pi+2 \theta, \alpha, \alpha+\beta, \alpha+n \beta \ldots$, respectively. Since the force on a dielectric object is given by the change in momentum of light induced due to refraction of the light by the object, the total force on the object is the difference between the momentum flux entering the object and that leaving the object.

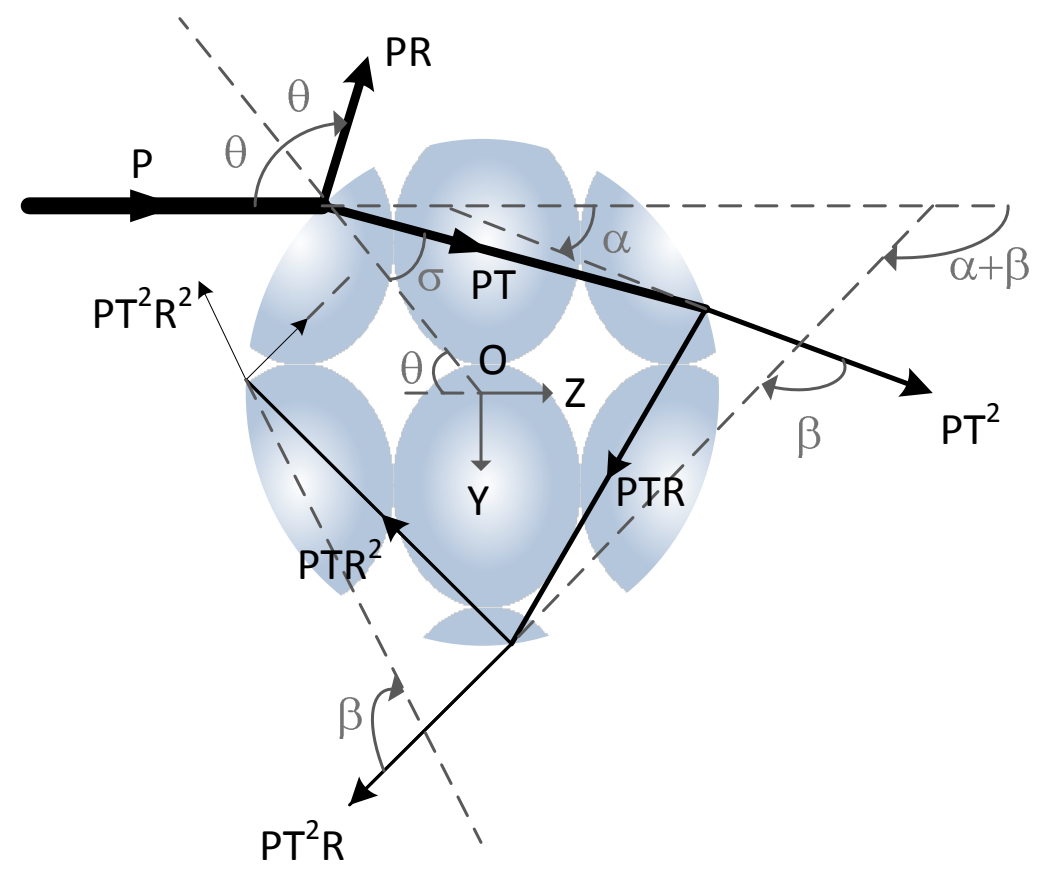

Figure 11: Geometry for calculating the force due to the scattering of a single incident ray of power $P$ by a dielectric sphere, showing the reflected ray PR and infinite set of refracted rays $P T^{2} R^{n}$. 
Therefore, the total force on the object is equal to:

$$
\overrightarrow{F_{T}}=\overrightarrow{M_{P}}-\left[\overrightarrow{M_{P R}}+\overrightarrow{M_{P T^{2}}}+\overrightarrow{M_{P T^{2} R}}+\cdots+\overrightarrow{M_{P T^{2} R^{n}}}\right]
$$

This total force can be centered on the origin of the sphere $\mathrm{O}$ and broken into $\mathrm{F}_{\mathrm{z}}$

(horizontal component) and $F_{y}$ (vertical component) as given by Roosen [Roosen, 1979] and Roosen and Imbert [Roosen, 1976]:

$$
\begin{aligned}
F_{z} & =\frac{n_{1} P}{c}-\left[\frac{n_{1} P R}{c} \cos (\pi+2 \theta)+\sum_{n=0}^{\infty} \frac{n_{1} P}{c} T^{2} R^{n} \cos (\alpha+n \beta)\right] \\
& =\frac{n_{1} P}{c}\left[1+R \cos 2 \theta+T^{2} \sum_{n=0}^{\infty} R^{n} \cos (\alpha+n \beta)\right]
\end{aligned}
$$

and

$$
\begin{aligned}
F_{y} & =0-\left[\frac{n_{1} P R}{c} \sin (\pi+2 \theta)+\sum_{n=0}^{\infty} \frac{n_{1} P}{c} T^{2} R^{n} \sin (\alpha+n \beta)\right] \\
& =\frac{n_{1} P}{c} R \sin 2 \theta-\frac{n_{1} P}{c} T^{2} \sum_{n=0}^{\infty} R^{n} \sin (\alpha+n \beta)
\end{aligned}
$$

In order to obtain a useful notation of these components (i.e. where the forces can be computed exactly), the total force in the complex plane is first calculated, $F_{\text {tot }}=F_{z}+i F_{y}$.

Thus:

$$
F_{\text {tot }}=\frac{n_{1} P}{c}[1+\mathrm{R} \cos 2 \theta]+i \frac{n_{1} P}{c} \mathrm{R} \sin 2 \theta-\frac{n_{1} P}{c} T^{2} \sum_{n=0}^{\infty} \mathrm{R}^{n} \mathrm{e}^{\mathrm{i}(\alpha+\mathrm{n} \beta)}
$$


The sum over $\mathrm{n}$ is a simple geometric series $\left(\sum_{n=0}^{\infty} a^{n}=\frac{1}{1-a}\right.$ for $\left.a<1\right)$, which simplifies the expression:

$$
F_{t o t}=\frac{n_{1} P}{c}[1+\mathrm{R} \cos 2 \theta]+i \frac{n_{1} P}{c} \mathrm{R} \sin 2 \theta-\frac{n_{1} P}{c} T^{2} e^{i \alpha}\left[\frac{1}{1-\mathrm{R} e^{i \beta}}\right]
$$

Rationalizing the complex denominator by multiplying the numerator and denominator by $1-\operatorname{R} e^{-i \beta}$, the denominator is no longer complex: $\left(1-\operatorname{Re}^{i \beta}\right)\left(1-\operatorname{Re}^{-i \beta}\right)=1+$ $R^{2}-R\left(e^{i \beta}+e^{-i \beta}\right)=1+R^{2}-2 R \cos \beta$.

And the total force becomes:

$$
F_{t o t}=\frac{n_{1} P}{c}[1+\mathrm{R} \cos 2 \theta]+i \frac{n_{1} P}{c} \mathrm{R} \sin 2 \theta-\frac{n_{1} P}{c} T^{2} e^{i \alpha}\left[\frac{1-R e^{-i \beta}}{1+R^{2}-2 R \cos \beta}\right]
$$

Isolating the real and imaginary parts of $F_{t o t}$, the force expressions for $F_{z}$ and $F_{y}$ become:

$$
\begin{aligned}
& F_{z}=\frac{n_{1} P}{c}\left\{1+R \cos 2 \theta-\frac{T^{2}[\cos \alpha+R \cos (\alpha-\beta)]}{1+R^{2}-2 R \cos \beta}\right\} \\
& F_{y}=\frac{n_{1} P}{c}\left\{1+R \sin 2 \theta-\frac{T^{2}[\sin \alpha+R \sin (\alpha-\beta)]}{1+R^{2}-2 R \cos \beta}\right\}
\end{aligned}
$$

Finally, using the geometric relations $\alpha=2 \theta-2 \sigma$ and $\beta=\pi-2 \sigma$, we get: 


$$
\begin{aligned}
& F_{z} \\
& =\frac{n_{1} P}{c}\{1+R \cos 2 \theta \\
& \left.-\frac{T^{2}[\cos (2 \theta-2 \sigma)+R \cos 2 \theta]}{1+R^{2}+2 R \cos 2 \sigma}\right\} \\
& F_{y} \\
& =\frac{n_{1} P}{c}\{1+R \sin 2 \theta \\
& \left.-\frac{T^{2}[\sin (2 \theta-2 \sigma)+R \sin 2 \theta]}{1+R^{2}+2 R \cos 2 \sigma}\right\}
\end{aligned}
$$

T and $\mathrm{R}$ depend on the polarization of the incident ray and are equal to:

- $\quad R_{S}=\left[\frac{\sin (\sigma-\theta)}{\sin (\sigma+\theta)}\right]^{2}$ and $T_{s}=1-R_{S}$, if the incident light is s-polarized

- $\quad R_{p}=\left[\frac{\tan (\sigma-\theta)}{\tan (\sigma+\theta)}\right]^{2}$ and $T_{p}=1-R_{p}$, if the incident light is $p$-polarized.

- If the incident light is unpolarized (containing an equal mix of $s$ - and $p$ polarizations), $R=\left(R_{s}+R_{p}\right) / 2$ and $T=1-R$.

These formulas take into account all scattered rays originating from the encounter of one incident ray with a dielectric sphere, and are therefore exact. In order to determine these forces, if the medium index of refraction $n_{1}$, the angle of incidence $\theta$, the light polarization, and the angle of refraction $\sigma$ using Snell's law are known, the exact forces imparted on the sphere by that ray can then be determined. 


\subsubsection{Forces imparted on a spherical object due to the laser beam}

As proposed by Ashkin [Ashkin, 1992], let's denote $F_{z}$ as the scattering force and name it $F_{s}$, and let's denote $F_{y}$ as the gradient force and name it $F_{g}$. As seen in Figure $11, z$ is in the direction of the incident ray and $y$ is perpendicular to it. Thus, the scattering force is always pointing in the direction of the incident ray and the gradient force is always perpendicular to it. This nomenclature arises from the Rayleigh regime expressions of the forces and is adopted in the Mie regime for the consistent labeling of forces regardless of the object's size.

The total force that the laser beam exerts on the spherical object can be calculated by integrating the single ray force expressions over the entire incident beam. The total scattering force $F_{S}$ is in the direction of the beam while the total gradient force, $F_{G}$, is highly dependent on the angular distributions of the rays. Incident rays with a large angle will provide a greater gradient force (as depicted in Figure 9) and therefore will contribute to a greater extent to the trapping force than rays with a smaller angle. Stable three dimensional trapping requires that the gradient force in the beam propagation direction is greater than all other forces (including the scattering force).

This is usually only possible with a high numerical aperture (N.A.) objective lens.

The overall trapping force is usually written as $F_{\text {trap }}=Q \frac{n_{1} P}{c}, \mathrm{Q}$ being the dimensionless trapping efficiency. It depends on many factors including the laser 
intensity configuration (Gaussian mode or else), the indices of refraction of the medium and the trapped object, and the size, shape, material properties of the trapped object. The maximum trapping force occurs for a particle placed along the incident laser beam axis, below the laser focus, where the scattering and gradient forces are in the same direction. It corresponds to the schematic depicted in Figure 9 A).

\subsubsection{CALIBRATION TECHNIIQUES: TRAP STIFFNESS DETERMINATION}

In general, an optical trap is modeled as a linear mechanical spring with the trap force $F_{\text {trap }}=k \Delta x$, where $\Delta x$ is the particle displacement from the trap center (actual focal point) and $k$ is the trap stiffness [Visscher, 1996]. This linear approximation is only valid for small particle displacements. To calculate the trap stiffness, the trap force is equated to a known applied force. Four methods to calculate trap stiffness are the Drag Force method, the non-linear Lateral Escape Force method, the Equipartition method and the Power Spectrum method [Svoboda, 1994], [Simmons, 1996], [Visscher, 1996].

The simplest of these approaches is the Drag Force method, where the static trap force is equated to the drag force associated with moving the trapped object through a fluid. This is particularly appropriate for this work and is the primary method employed as the drag force is well defined for a rigid solid sphere. For highly nonspherical and/or biological objects, the drag force method alone may not be sufficient, therefore additional trap calibration methods may be necessary. 
From low Reynolds Number (Re) hydrodynamics, the drag force for a stationary sphere in a moving fluid between two infinite parallel plates (a good approximation for the high aspect ratio microchannels in this experimental study) is given by (Happel, 1983]:

$$
F_{\text {drag }}=\frac{6 \pi \mu a v}{1-1.004\left(\frac{a}{l}\right)+0.418\left(\frac{a}{l}\right)^{3}+0.21\left(\frac{a}{l}\right)^{4}-0.169\left(\frac{a}{l}\right)^{5}}
$$

where $a$ is the radius of the spherical particle, $l$ is the half height of the channel, $\mu$ is the fluid viscosity, and $v$ is the fluid velocity experienced by the sphere. This modified Stokes drag accounts for particle-wall effects associated with the finite plate separation. The trap stiffness is then calculated by linearly fitting a range of drag force $\left(F_{\text {drag }}\right)$ versus displacement data $(\Delta x)$ (the difference between the particle position when the particle is trapped without flow and trapped with flow) and determining the slope.

In the non-linear Lateral Escape Force method, the trapped object is displaced at different velocities, as it was for the drag force method. The force exerted on the particle can be calculated knowing the velocity and calculating the drag coefficient. The minimum force necessary to remove the particle from the trap is the escape force. It corresponds to the maximum trap force, and the trap stiffness can be deducted using the spring approximation. 
The equipartition method uses the properties of Brownian motion. By monitoring the random motion of the trapped object due to temperature and calculating the variance $\langle\Delta x\rangle$, the trap stiffness is calculated as $k=\frac{k_{B} T}{\langle\Delta x\rangle^{2}}$, where $k_{B}$ is the Boltzmann's constant and $T$ the temperature. For this technique, the position detector needs to be well calibrated and precise, as any error is squared.

For the last method, the power spectrum of the trapped object's motion is calculated and a Lorentzian fitted to the data. The corner frequency, $f_{0}$, of the Lorentzian can be used to calculate the trap stiffness if the drag coefficient of the trapped object is known.

\subsubsection{APPLICATIONS}

Researchers have applied OT to study kinesin motor motility [Asbury, 2003], manipulate biological structures [Svoboda, 1994], and order suspended particles [Polin, 2005]. Knöner et al. [Knoner, 2005] characterized the velocity near a spinning object by suspending a 1 micron particle with OT then tracking the particle motion after trap cessation in a particle tracking technique. Leonardo et al. [Leonardo, 2006] extended this technique to multi-point measurement with holographic OT. While these techniques are capable of measuring velocities throughout a three-dimensional (3-D) microchannel, the pointwise nature of the technique limits simultaneous fluid velocity measurements to a few (a reported maximum of 8 points measured simultaneously) 
locations within the field of view. This may not provide sufficient detail to accurately determine the fluid stresses surrounding a suspended object. In addition, the resolution of the velocity measurement is limited by the trapped particle size, which is greater than a micron $(1 \mu \mathrm{m}$ for Knöner et al. [Knoner, 2005] and 1.1 $\mu \mathrm{m}$ for Leonardo et al. [Leonardo, 2006]).

\subsection{MICRON RESOLUTION PARTICLE IMAGE VELOCIMETRY}

Micron-resolution particle image velocimetry ( $\mu \mathrm{PIV}$ ) is a two-dimensional (2-D) full field velocity measurement technique [Santiago, 1998] capable of resolving velocity fields to within $436 \mathrm{~nm}$ of a microchannel wall [Tretheway, 2002]. Velocity measurements are obtained by seeding the flow domain with fluorescent nanoparticles, volume illuminating the region of interest by a double pulsed-laser, and imaging the emitted light provided by the excited nanoparticles at two different times using a digital camera synchronized with the pulses of the laser. For each pair of laser pulses two images are obtained. Note, the laser cannot be continuously illuminating the nanoparticles as their excited state lasts for a finite time, typically 1-10 ns, and then photobleach. The emission light level is affected by the nanoparticles concentration, particle size, illumination wavelength, pulse energy, pulse duration, filters, and the medium in which the nanoparticles are seeded. 
In principle, the resulting image pairs are then cross-correlated as sketched in

Figure 12 . The local velocity of the fluid $\vec{v}$ is then obtained by dividing the measured

displacement, by the time delay, $\vec{v}=\overrightarrow{\Delta x} / \Delta t$.

However in practice, multiple velocities may be determined from a single image pair by first dividing each image into a grid of uniform interrogation regions of size $p \times q$ pixels. Following Wereley et al. [Wereley, 2005] a simple correlation function at a certain interrogation region is usually represented as:

$$
\Phi_{k}(m, n)=\sum_{j=1}^{q} \sum_{i=1}^{p} f_{k}(i, j) \cdot g_{k}(i+m, j+n)
$$

where $f_{k}(i, j)$ and $g_{k}(i, j)$ are the gray value distributions of the first and second images, respectively, of the $\mathrm{k}^{\text {th }}$ image pair. The correlation function for an image pair has a peak at the position of the particle image displacement in the interrogation area.

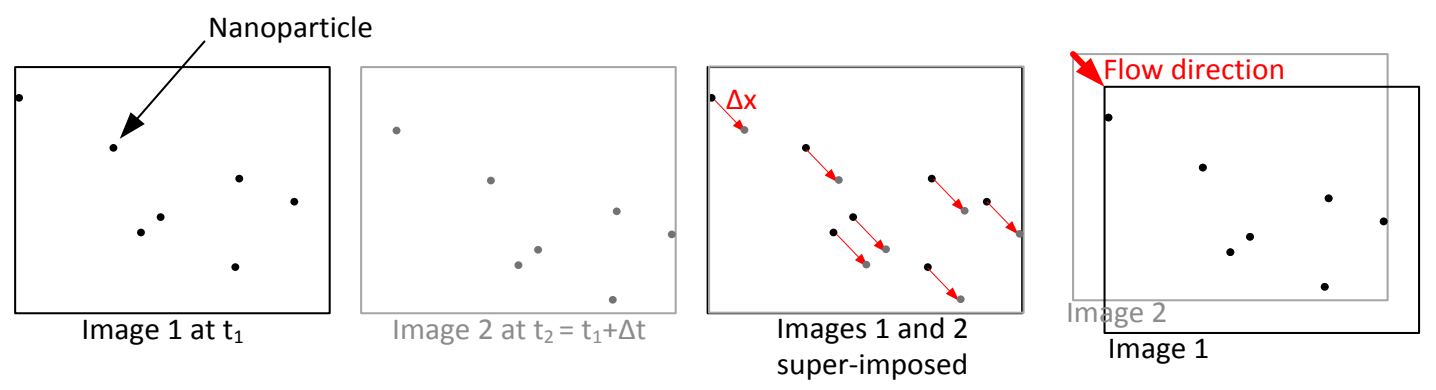

Figure 12: Simplified cross-correlation schematics: nanoparticles move with the flow and can be visualized at two different times in order to obtain the flow direction and velocity field in the entire field of view. 
If there is more than one particle in an interrogation area that is visible in both of the image pairs, the peak should be the highest among all the peaks of $\Phi_{k}$ (Figure 13 A)). The sub peaks, which result from noise, i.e. particles coming and going in and out of the interrogation region, are usually lower than the main peak. However, when the interrogation window does not contain enough particles, the main peak can be lower than the sub peaks, and as such, an erroneous velocity vector is generated. Because the flow is steady and laminar, its velocity profile is constant. Therefore the main peak is always at the same position for different image pairs, while the noise, by definition, varies. Therefore to increase the signal to noise ratio of the velocity measurements, paired laser pulses can be repeated $\mathrm{x}$ number of times to acquire $\mathrm{x}$ image pairs with the same time lapse (within nanoseconds) $\Delta$ t between each image of the image pairs. The image pairs can be correlated, $\Phi_{k}(m, n)$, and the correlations ensemble averaged over a large number of PIV recording pairs (N). The averaged (or ensemble) correlation function is given as:

$$
\Phi_{\mathrm{ens}}(\mathrm{m}, \mathrm{n})=\frac{1}{\mathrm{~N}} \sum_{\mathrm{k}=1}^{\mathrm{N}} \Phi_{\mathrm{k}}(\mathrm{m}, \mathrm{n})
$$



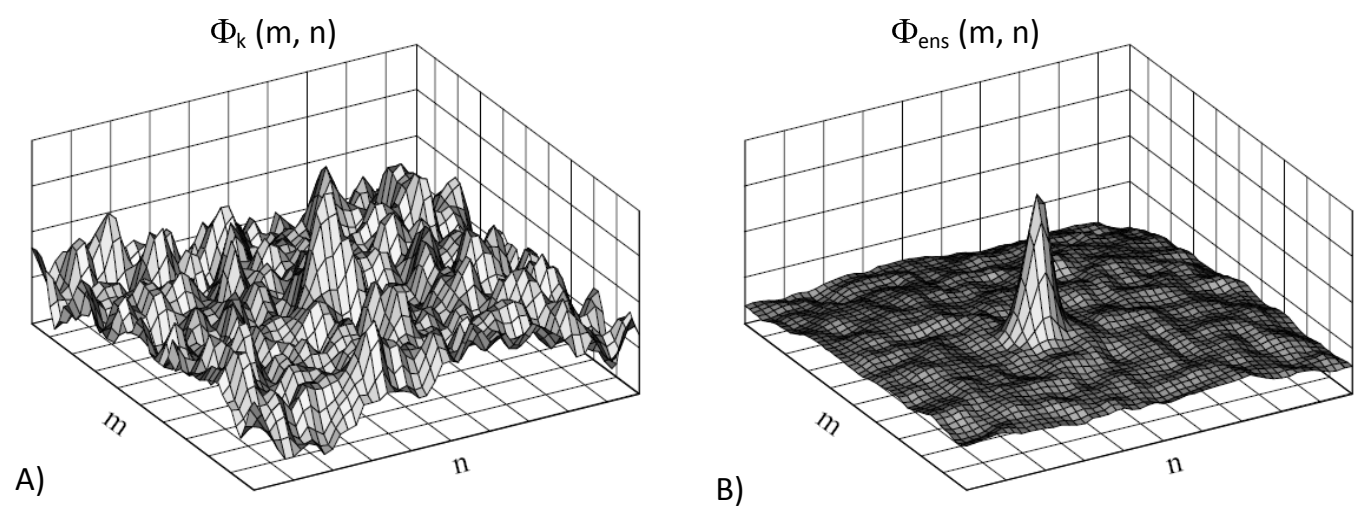

Figure 13: Effect of ensemble correlation: A) results with conventional correlation for one of the PIV recording pairs; B) results with ensemble correlation for over 100 PIV recording pairs [Wereley, 2005].

In this process, the main peak will remain at the same position in each correlation function but the noise peaks, which occur randomly, will average to zero (Figure 13 B)). For example, in an interrogation area where there are few particles, the particles will correlate and contribute to the displacement peak. However, the noise peaks due to particles going in and out of plane will also be quite large. Using the next image pair, the correlation map is computed for the same interrogation area and added to the ensemble correlation map. Each particle pair added increases the height of the displacement peak. Each non-paired particle increases a random noise peak. As the correlation maps from more images are summed, the signal to noise ratio in the correlation peak increases as shown in Figure 13. Thus, the more image pairs, the cleaner the vector field. 
Finally, the particles' displacement is obtained from the location the primary correlation peak and the velocity calculated by dividing the displacement by the time lapse between laser pulses. With a CCD camera with 1000's of pixels and common interrogation regions of $16 \times 16$ pixels, velocities at thousands of locations across the entire field of view can be obtained simultaneously. Flow properties such as shear rates can then be obtained by differentiating the velocity field throughout the flow region.

Recently, this technique has been used extensively in microfluidics for characterization of flows in microchannnels, BioMEMS, and flow around cells. While the technique was initially developed for microscale velocity measurements, it has been extended to measure wall positions with tens of nanometers resolution [Stone, 2002] and the deformation of hydrogels [Olsen, 2000]. In our project, the determination of the velocity field is crucial. For a trapped object, suspended in a fluid flow, the $\mu$ PIV technique enables us to obtain the entire velocity field around a trapped object and thus calculate the applied local fluid stresses. We can therefore obtain global fluid stress through force measurements of the optical tweezers and simultaneously measure the local fluid stresses from the velocity field obtained with $\mu$ PIV. 


\section{CHAPTER 3}

\section{INSTRUMENT DEVELOPMENT}

Two laser-based techniques, micron-resolution particle image velocimetry ( $\mu$ PIV) and optical tweezers (OT), were integrated to form the $\mu$ PIVOT. Optical tweezers allow the capture, suspension, and manipulation of polystyrene or glass microspheres, single cells or biomolecules by optical gradient forces. $\mu$ PIV can measure local fluid movements including that of steady or transient shear and extensional flows in addition to providing high resolution imaging of cellular deformation. The combination of these two techniques through a single inverted microscope provides a unique platform for characterizing cellular biomechanics (stress-strain) as a precursor to deciphering the mechanotransduction phenomenon. The designed instrument enables a new realm of microscale cell studies by allowing a sequence or combination of mechanical stresses to be applied to the same individual cell while measuring the deformational response for each stress state. This technique eliminates the effects of mechanical restraints associated with many experimental approaches by applying an optical force (contact region of $\approx 1 \mu \mathrm{m}$ diameter) that can be attached/detached by shuttering the OT laser. It is a single cell approach that extends the range of applicable fluid induced stresses to include extensional stresses that cannot be generated in cell cultured monolayer shear stress studies. 
The distinct wavelengths of each laser allow the integration of both techniques through a single inverted microscope. A set of specific filter cubes placed within the microscope allows this combination to be possible. The lasers are introduced in the microscope through two different optical ports. The OT system incorporates a series of optical components arranged on an optical table to control the position, direction, intensity and beam size of the laser (as explained in the paragraph below). The beam is aimed at the bottom entry port positioned at the back of the microscope. The $\mu$ PIV laser is introduced into the microscope through the top entry port using a fiber optics cable.

This chapter first describes the materials, methods, and assembly of the $\mu$ PIVOT focusing on three separate systems: the microscope, optical tweezers, and $\mu$ PIV. Improvements added to automate the system are discussed.

\subsection{MICROSCOPE}

The microscope used to implement the combined instrumentation requires the following characteristics: multiple entry ports for the combination of the different laser techniques at the same sample location, multiple carrousels for the stacking of different filters (as described below), the possibility of automating the entire system, and an inverted framework for ease of viewing and setup. The microscope chosen initially for the $\mu$ PIVOT integration was the Nikon TE2000U. 
The main objective lens used for this project is the Plan Apochromat TIRF 60X Oil, N.A. 1.45. The term "Plan" stands for flat field, which means that it is corrected for the center of the field and the outer edges are in focus. The term "Apochromat" makes it the most highly color corrected objective lens on the market. This special lens also has a temperature correction mechanism, in order to be efficient not only at room temperature but also at $37^{\circ} \mathrm{C}$ (human body temperature) if necessary. The Numerical Aperture (N.A.) gives a measure of two important objective characteristics, namely its light-gathering ability and its aptitude to resolve fine details in a specimen. Each characteristic is important for our system. The numerical aperture is defined as N.A. = $\mathrm{n} \cdot \sin (\theta)$, where $\mathrm{n}$ is the refractive index of the objective's working medium (for oil immersion objectives, $\left.\mathrm{n}_{\mathrm{oil}}=1.515\right)$, and $\theta$ is the half-angle of the light collecting cone. As seen in the overview on the theory of optical tweezers, the bigger the angle, the better the optical trapping efficiency. Fortuitously, the higher resolving power that comes with high N.A. is an important feature for $\mu$ PIV measurements. The resolving power is

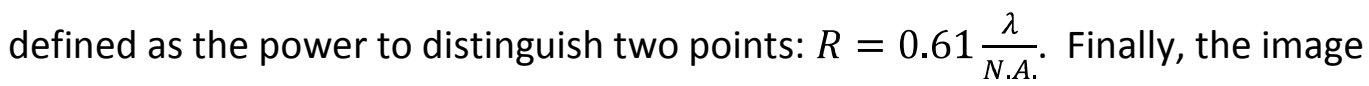
brightness (B) is also directly correlated to the N.A.. It is determined for transillumination by the square of the ratio between the N.A. and its magnification factor $(\mathrm{M}): B_{\text {trans }} \propto\left(\frac{N \cdot A \cdot}{M}\right)^{2}$. For epi-illumination however, the objective also functions as a condenser for the illuminating light, introducing another factor of N.A. ${ }^{2}$ in the 
numerator which yields: $B_{e p i} \propto \frac{N \cdot A^{4}}{M^{2}}$. Hence, in epi-illumination, the image brightness $B_{\text {epi }}$ varies proportionally to the fourth power of N.A.. In $\mu$ PIV, where the fluorescent signal from tracer particles is weak, a high N.A. objective is important. Finally, the higher N.A., the shallower the depth of focus (DOF): $D O F=\frac{n . \lambda}{2 N . A .}$, with $\mathrm{n}$, the index of refraction of the medium (for oil, $\mathrm{n}_{\mathrm{oil}}=1.515$ ) and $\lambda$, the laser's wavelength (for the $\mu$ PIV laser, $\lambda=532 \mathrm{~nm}$ ). A shallow DOF results in less noise coming from fluorescing particles that are not in the plane of focus, providing a better image quality.

\subsection{OPTICAL TWEEZERS}

The basic system to obtain optical trapping is relatively simple to construct. One needs a laser source, preferably with a wavelength as mildly harmful as possible to biological cells (if the object to be trapped is a cell), a microscope with an objective lens of high numerical aperture to focus the rays to the focus point (as described in the previous chapter), and a means of controlling the optical trap, the simplest way being the use of convex lenses in the optical path before entering the microscope. Small adjustments are then added to the system for stable and reproducible experiments.

The detailed experimental setup is depicted in Figure 14 and shown in Figure 15. 


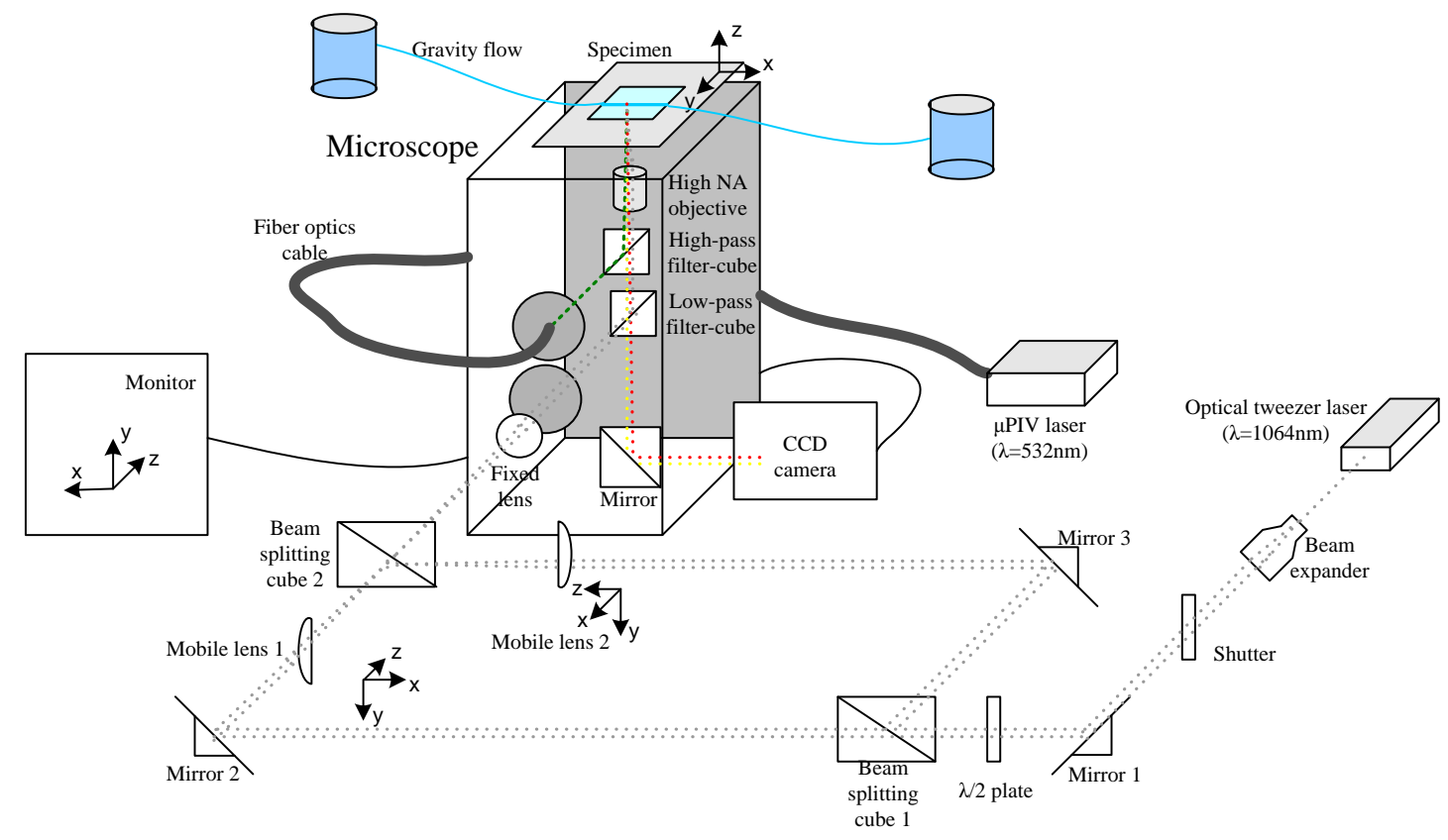

Figure 14: Schematics of the $\mu$ PIVOT.

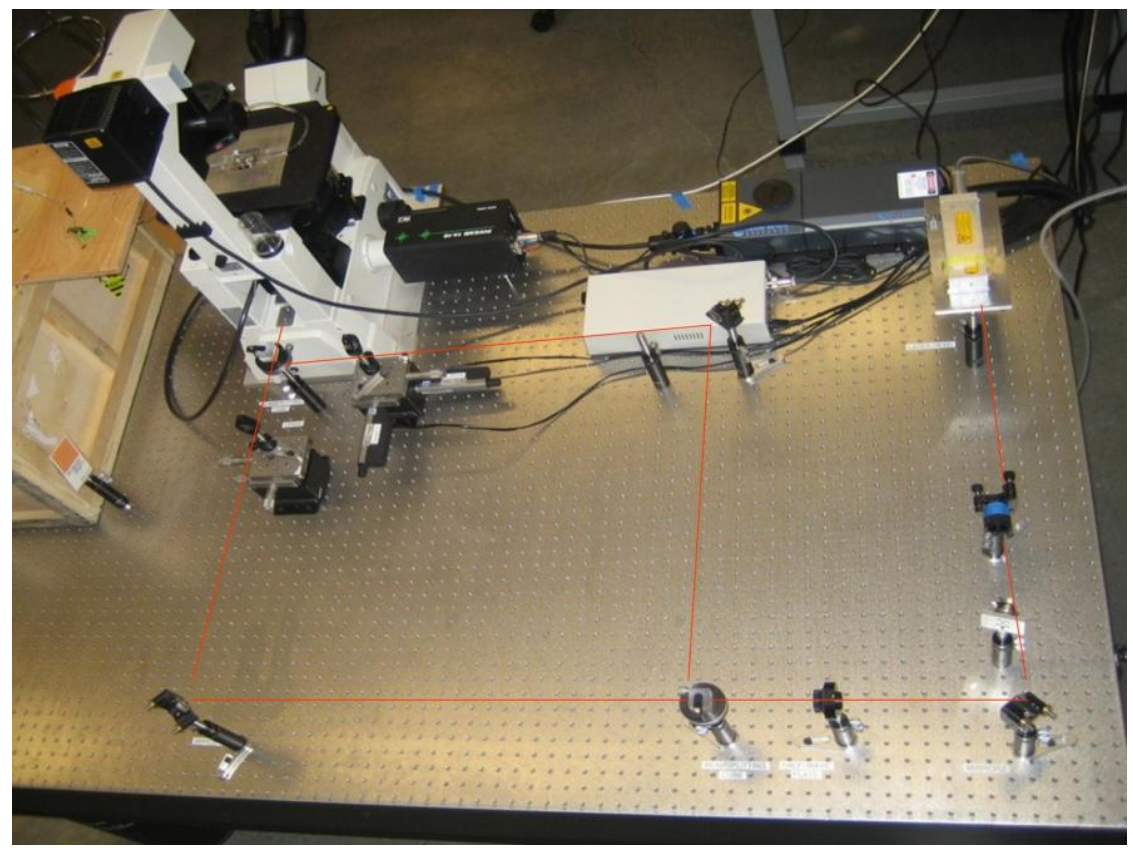

Figure 15: Picture of the $\mu$ PIVOT set up. The red lines indicate the path taken by the OT laser beam. 
The OT system was constructed on an optical table (Newport R4000, Irvine, CA) with laminar flow isolation (Stabilizer I-2000 Series, Newport) in order to limit vibrations transmission from the building. A $1064 \mathrm{~nm}$ wavelength laser (BL-106C, J series, Spectraphysics, Mountain View, CA) provides the trapping light source, with a maximum power of $5.4 \mathrm{~W}$. The power is monitored through a customized Labview program controlling the laser source's current input (minimum firing current input of $8 \mathrm{~A}$ ). The $1064 \mathrm{~nm}$ wavelength is known to be minimally absorbed by biological material and therefore to be relatively harmless to the cells [Svoboda, 1994]. The laser beam, in order to trap efficiently, needs to slightly overfill the back aperture of the objective lens (measured diameter of $13 \mathrm{~mm}$ ). The waist diameter of the beam exiting the laser head is $350 \mu \mathrm{m}$, and the beam divergence is 2.22 radians (half angle). For the optical path length, this divergence is insufficient to overfill the back aperture, therefore the laser beam is initially expanded through a $2 x$ beam expander (BE02X-C, Thorlabs, Newton, NJ) that doubles the beam waist diameter while keeping it collimated. Following the beam expander, a safety shutter (SH-10, Electro-Optical Products Corp., Glendale, NY) linked to a foot pedal (Treadlite II T-91-D, Linemaster Switch Corp., Woodstock, CT) is inserted to block the laser beam when necessary. As an additional feature, two traps can be generated from the single beam, which broadens the applications possible. As an example, we can stretch the cell by trapping it at two ends, or trap two different cells close to one another to study their interactions. In order to split the laser into two 
controlled beams before entering the microscope, the expanded beam is directed by a mirror (10QM20HM.15, Newport) into a quartz half-wave plate (10RP12-34, Newport).The half-wave plate rotates the plane of polarization from a polarized laser to any other desired plane. The $\mathrm{Nd}: \mathrm{YVO}_{4}$ laser is vertically polarized, and to obtain a $45^{\circ}$ angle polarization, the half-wave plate is simply placed at half that angle $\left(=22.5^{\circ}\right)$ to the vertical. The beam is now linearly $45^{\circ}$ polarized, which implies that there is an equal magnitude of $s$ and $p$ polarization components before going through a polarizing cube beamsplitter (05BC16PC.9, Newport). Considering a coordinate system using the plane made by the propagation direction and a vector normal to the plane of the cube reflecting surface (plane of incidence), the component of the electric field parallel to this plane is termed $p$-like (parallel) and the component perpendicular to this plane is termed s-like (from senkrecht, German for perpendicular). As shown on Figure 16 , the beam is split into two independent and equally intense traps (s component of the beam for one, and $\mathrm{p}$ component for the other) at the specimen.

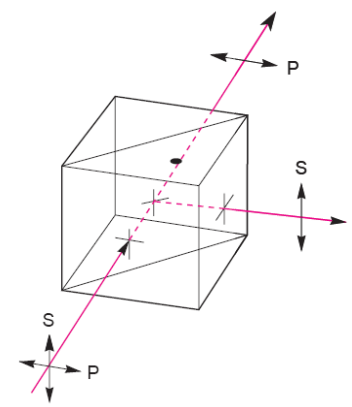

Figure 16: Transmission of p-polarized light and reflection of s-polarized light through a cube beam splitter (image from Newport Corp). 
Note: the system is set to provide two equally powered traps that could have been provided by using a half/half non polarized beam splitting cube. However, the polarized configuration allows for unequal splitting of the beam if desired.

The resulting two beams are directed by separate mirrors (same as above) through plano-convex lenses (BK7, LA1433-C, Thorlabs) positioned on vertical and linear stages (MVN80 and UMR8.25, Newport). The stages provide a 3-D translation range of $25 \mathrm{~mm} \times 25 \mathrm{~mm} \times 12.5 \mathrm{~mm}$ per beam. The beams are steered by rotation of the direction of the laser beam propagation in a plane conjugate to the back focal plane of the objective. This is achieved by controlling the position of the first lens of a 1:1 telescope. For movements in the focal plane as shown in Figure 17D), rotations are created at the back aperture of the objective lens (and then transmitted to the sample). The two lenses are separated by a distance of $f_{m}$ (focal length of the mobile lens) $+f_{f}$ (focal length of the fixed lens) in order to keep the beam collimated. The distance to the objective lens is given by $y=\left(f_{m}+f_{f}\right) f_{f} / f_{m}$. For a 1:1 telescope, $f_{f}=f_{m}=f, y=2 f$ and the fixed lens has therefore been positioned at a distance of $2 \mathrm{f}$ from the back aperture of the objective lens (the lenses used are BK7 C-coated plano convex lenses with a focal length of $150 \mathrm{~mm}$, model LA1433-C, Thorlabs). For axial movements of the trap, slight adjustments of the beam collimation can be used by changing the axial position of the mobile lens. 


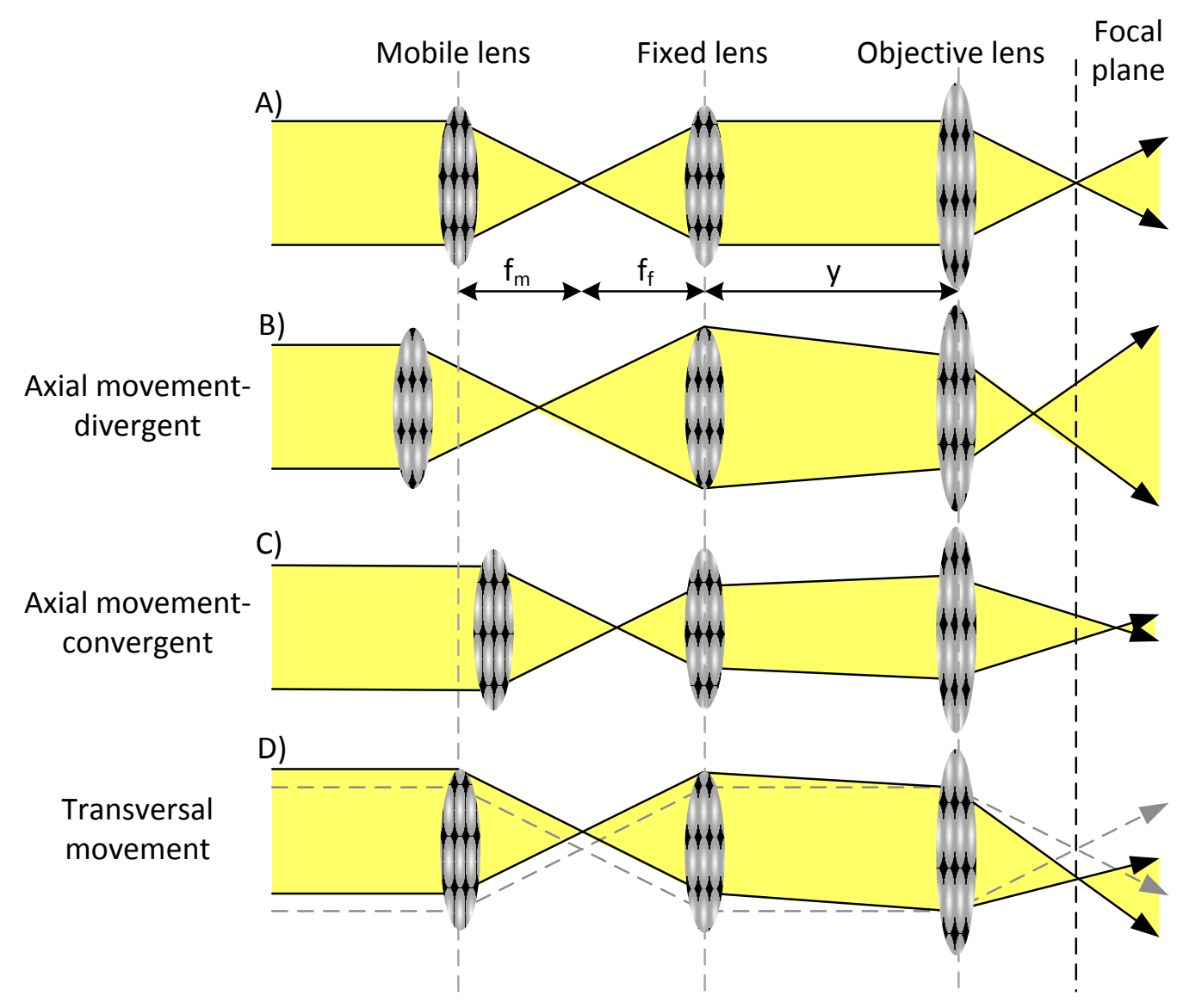

Figure 17: Demonstration of trap movement through movement of a mobile lens. Translation of the mobile lens axially or transversally causes a displacement of the focus point at the sample.

As shown in Figure 17B), to bring the trap focus closer to the objective lens, the mobile lens is placed further away from the fixed lens. Inversely, to send the trap further away from the objective lens, the mobile lens is brought closer to the fixed lens (Figure 17C). Using this simple telescope method, the optical trap can therefore be steered in three dimensions using the translation of a single lens. This method allows for a wide translation capability (effectively the field of view) of a trapped particle within a 
channel. The position of the stages on which the moveable lenses are placed is controlled through manual micrometers (BM17.25, Newport) with a resolution of $1 \mu \mathrm{m}$. The translation stage resolution coupled with the 1:1 telescope and optical magnification of the microscope objective allows sub-micron position movement and object resolution at the sample. Before entering the fixed lens, the two independently controlled beams are recombined into a single path by a second polarizing cube beamsplitter. As shown in the schematic in Figure 14, once in the microscope the infrared beams reflect off a low pass filter (cut-off wavelength of $1000 \mathrm{~nm}$ ), pass through a high pass filter (cut-on wavelength of $550 \mathrm{~nm}$ ) and are focused through the objective lens into the sample. Figure 18 shows the main wavelengths used in the $\mu$ PIVOT system and their transmission through the different filter cubes.

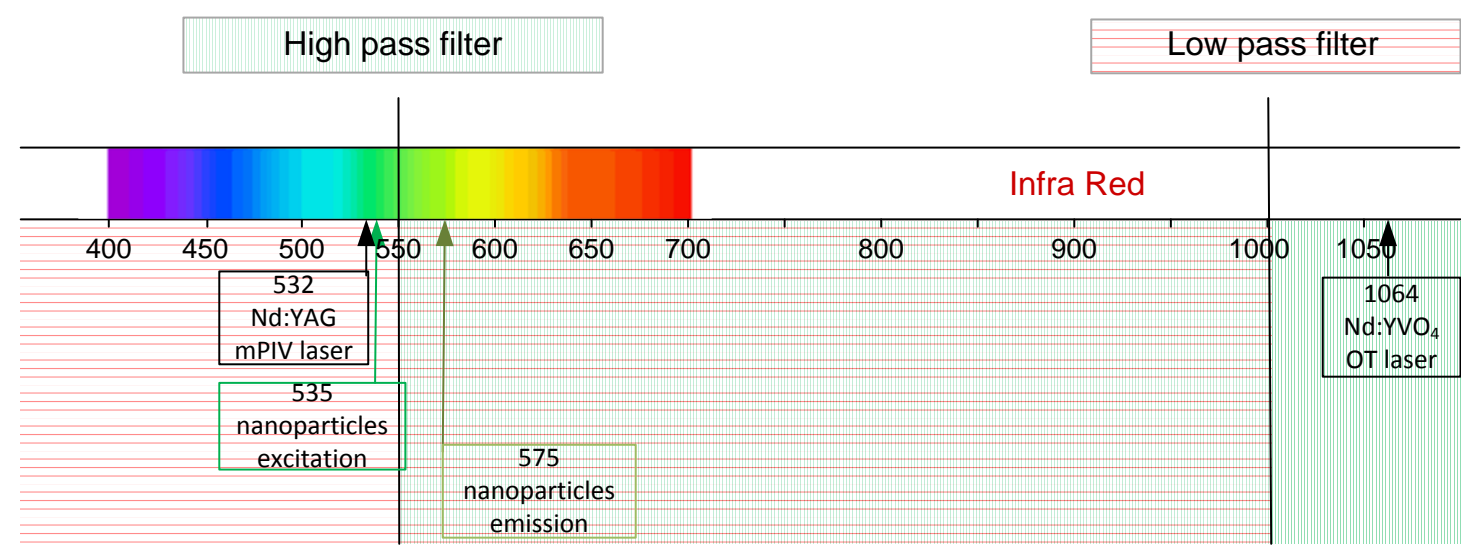

Figure 18: Key wavelengths (in $\mathrm{nm}$ ) of the $\mu$ PIVOT system. The stripe colors indicate the transmission through the filters. Any light beam having a wavelength in the horizontal stripes zone goes through the low pass filter, but reflects off the high pass filter. Inversely, any light beam having a wavelength in the vertical stripes zone goes through the high pass filter but reflects off the low pass filter. If in the grid zone, the light beam goes through both of the filters. 
Once a particle is trapped by the OT, its position and shape deformation may be imaged with either a moderate frame rate, low resolution ( 1 pixel $=125 \mathrm{~nm}$ ) CCD camera (Gevicam GP-3360 running a visualization imaging software: Coyote V2.3.0, Gevicam Inc.), or the high resolution $\mu$ PIV camera (1 pixel = $109 \mathrm{~nm}$ ) described in the next section. Captured images are further analyzed with object tracking software (Spotlight-8, NASA) and an image analysis freeware (Gimp V2.6.6) for characterizing position and size/deformation of trapped particles with a resolution of $+/-0.5$ pixels.

\subsection{MICRON RESOLUTION PARTICLE IMAGE VELOCIMETRY}

$\mu \mathrm{PIV}$ can measure local fluid movement including that of steady or transient shear and extensional flows in addition to providing high resolution imaging of cellular deformation. The $\mu$ PIV system consists of two $532 \mathrm{~nm}$ wavelength, $3.5 \mathrm{~mm}$ diameter, Qswitched Nd:YAG lasers (Solo III, 15Hz, New Wave Research, Fremont, CA) connected to the upper optical port of the inverted microscope by a beam expanding fiber optic cable. The very short laser pulses (3-5 ns duration, with a maximum energy of $50 \mathrm{~mJ}$ per pulse) are reflected by the high pass filter cube noted previously and directed into the objective lens to illuminate the flow field which is seeded with $275 \mathrm{~nm}$ diameter fluorescent polystyrene spheres (nile red fluorescent 535/575, carboxylate-modified, Molecular Probes, Carlsbad, CA) at a concentration of $50 \mu \mathrm{L}$ of nanoparticles solution ( $2 \%$ solids) to $2 \mathrm{~mL}$ of distilled water. This concentration provides relatively good images for cross-correlation. The concentration is sufficient to provide adequate 
particle signals per interrogation region yet low enough that out-of-focus particles do not overwhelm the image with background noise. The fluorescent light emitted by the nanoparticles $(\lambda=575 \mathrm{~nm})$ is captured by the objective lens, passes through both the low and high pass filter cubes, and is focused onto a $1376 \times 1024$ pixel ( $8.88 \mathrm{~mm} \times 6.60 \mathrm{~mm}$ ) CCD camera (PIVCAM 14-10 Model 630055, TSI, Shoreview, MN). The filter cube filters have been selected to maximize the fluorescent image intensity while removing the 532 $\mathrm{nm}$ laser light through dichroic coatings that reflect and transmit wavelengths selectively. The image pairs are viewed and saved using data acquisition, analysis and display software (Insight 3G, TSI). The data processing includes the use of a viewer (IrfanView freeware, Wiener Neustadt, Austria) to convert images to processcompatible formats, a customized program for $\mu$ PIV interrogation (written in Matlab, The Mathworks, Natick, MA), a PIV vector validation software (CleanVec V1.13.41, freeware), and finally a software for viewing flow images, displaying the calculated vector fields, and mapping the flow streamlines (Tecplot 10, Tecplot Inc., Bellevue, WA). The field of view with a $60 x$ objective and a $1 \times$ projection lens is $0.15 \times 0.11 \mathrm{~mm}$.

\subsection{AUTOMATION OF THE SYSTEM}

\subsubsection{NEW FEATURES}

In order to automate the system and add features to the existing set up, several components were modified. The microscope was upgraded to the Nikon TE $2000 E$ 
microscope and a Prior Pro Scan II (H117) automated stage was installed. This step up increases the position resolution and more importantly allows better control of uniform flow velocities compared to gravity driven channel flows. Using the automated stage to generate fluid motion relative to the particle also reduces the setup time before each experiment (from several hours to 20 minutes). The translation movements of the lenses controlling the two traps were also computerized, using motorized actuators (LTA-HL, Newport) linked to a controller (XPS-C6, Newport) via drivers (XPS-DRV01, Newport) for all three $\mathrm{x}, \mathrm{y}$ and $\mathrm{z}$ translations.

Confocal microscope capabilities (Hyphenated Systems model 3DMAP), with a 120-watt short arc lamp fluorescent illumination (X-cite 120 PC, Exfo) were combined with the $\mu$ PIVOT system. This addition opens the door to a wide range of new research possibilities such as fluorescent microscopy of living cells, determination of the multiaxial stress applied to a trapped object by measuring $\mu$ PIV velocities in multiple planes around the cell (versus one-plane measurements), and determination of the exact geometry of microfluidic channels/devices in 3-D. The confocal microscope system features a digital read out of the focal plane position and a digital focus knob that increase the axial position resolution (app. $0.1 \mu \mathrm{m}$ ) for any application using the microscope. 


\subsubsection{MODIFICATIONS}

The principal modifications needed when upgrading the system were due to the addition of the confocal system which increased the height of the microscope by approximately $150 \mathrm{~mm}$. Taking advantage of this system remodeling, other non-related modifications were made such as the switching of the entry ports between OT and $\mu$ PIV system. The axial movement of the trap is controlled by the moveable lens' relative position to the fixed lens. As described above, for a perfectly collimated beam and optimum trapping efficiency, this distance, as well as the distance between the fixed lens and the objective lens is equal to $2 f$ when the beam is collimated. However, it has been observed experimentally that small movements at the sample require significant movement of the moveable lens. In the initial configuration the required movement was beyond the limits of the translation stages. A shorter focal length reduces the required movement and hence improves the system. A focal length of $100 \mathrm{~mm}$ was chosen for all the lenses (model LA1509-C). This focal distance enables reasonable movement of a trapped particle within the limits of the translation stage. As shown in Figure 19, in order to achieve the distance required between the fixed lens and the objective lens, the OT entry port was switched to the top entry port, the $\mu$ PIV port to the bottom, and the fixed lens mounted inside the microscope housing (the corresponding filter cubes were exchanged inside the microscope as well). 

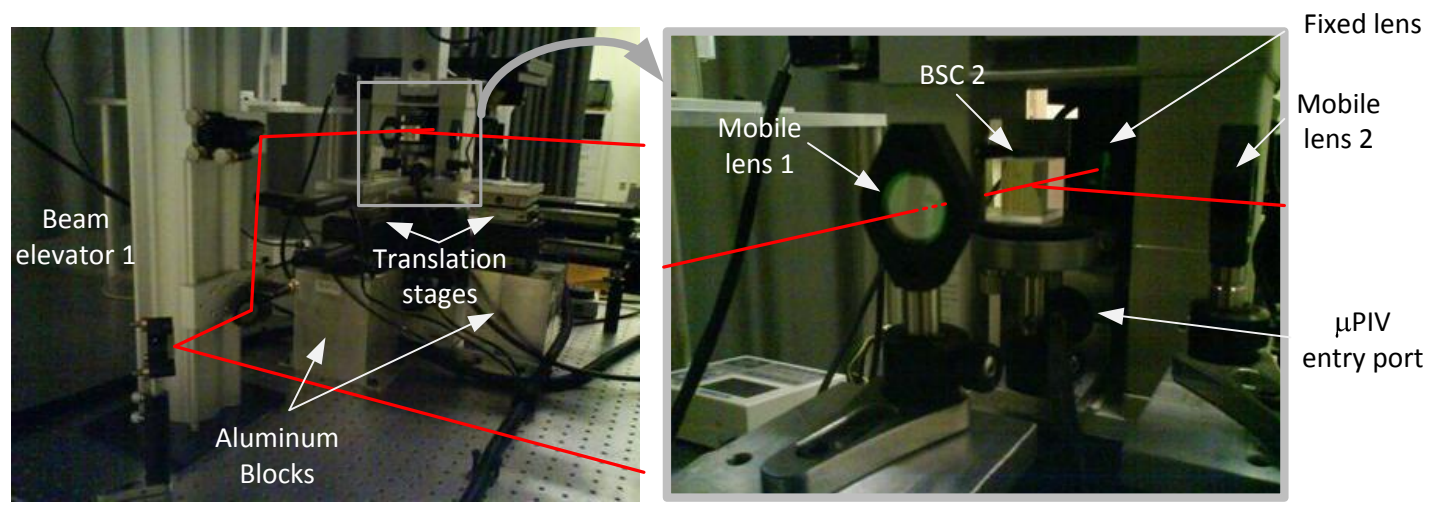

Figure 19: Close-up pictures of the current $\mu$ PIVOT configuration. A) addition of beam elevators and aluminum blocks to raise the OT optical path. B) $\mu$ PIV entry port is below the OT entry port.

Additionally, to maintain the $2 \mathrm{f}$ distance between the fixed lens and each mobile lens, the mobile lenses needed to be placed very close to the recombining beam splitting cube (BSC2). As shown in the SolidWorks drawing in Figure 20, aluminum plates were designed to hold the lenses on top of the translation stages close to the BSC2. The optical paths were raised using beam elevators prior to the beam going through the mobile lenses, and elevating the mobile lenses/translation stages system by placing them on stable aluminum blocks. This bulk setup allowed for a stable raise of the system without introduction of vibrations.

Figure 21 shows the modified setup while Figure 22 provides a schematic of the system. 

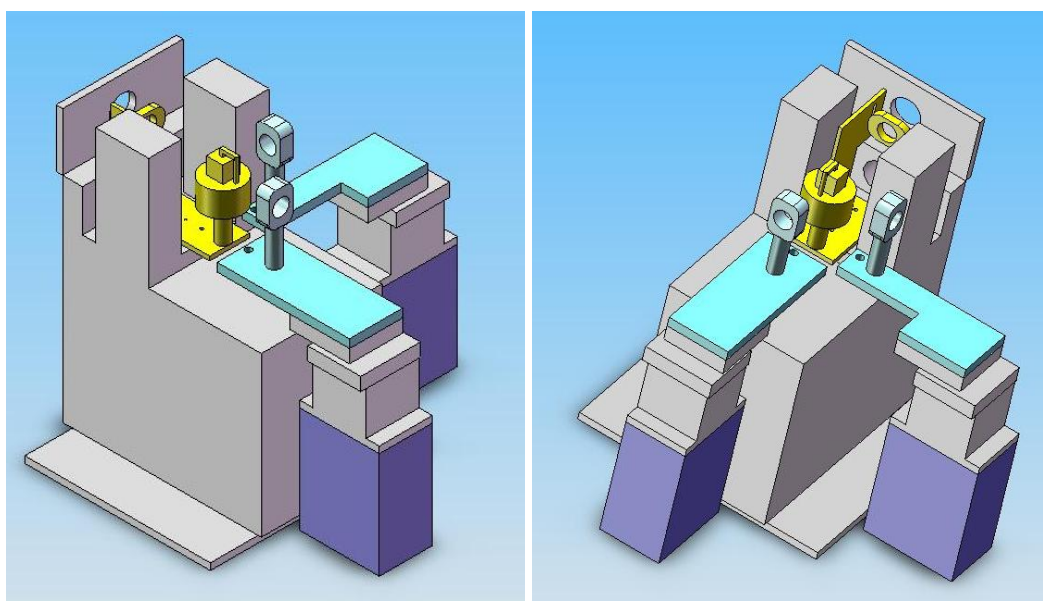

Figure 20: Solid Works representation of the elevation of the OT system: Gray features represent the back of the microscope and the translation stages. Colored components are the custom built features added: Aluminum blocks (dark blue) were positioned under the translation stages, aluminum plates were added to position the lenses closer to the fixed lens (light blue), a holder (yellow) was designed to hold the beam splitting cube and the fixed lens inside the microscope.

Aluminum blocks

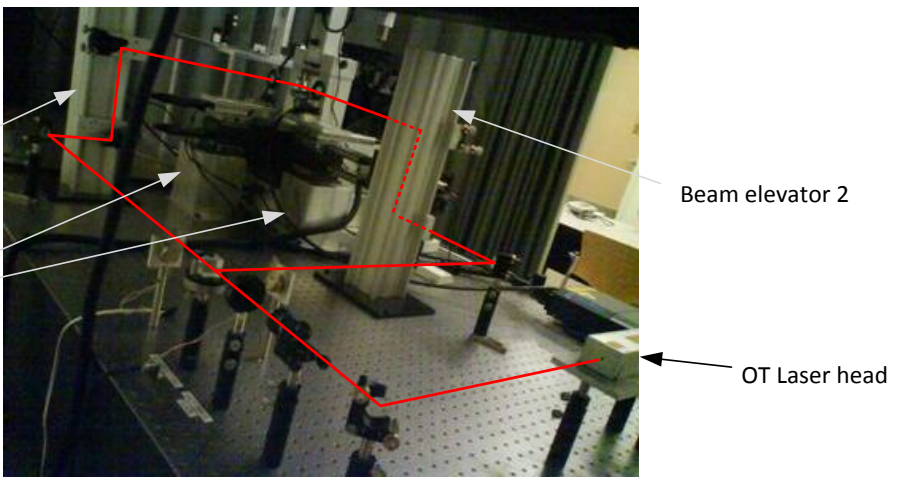

Figure 21: Picture of the modified setup. 


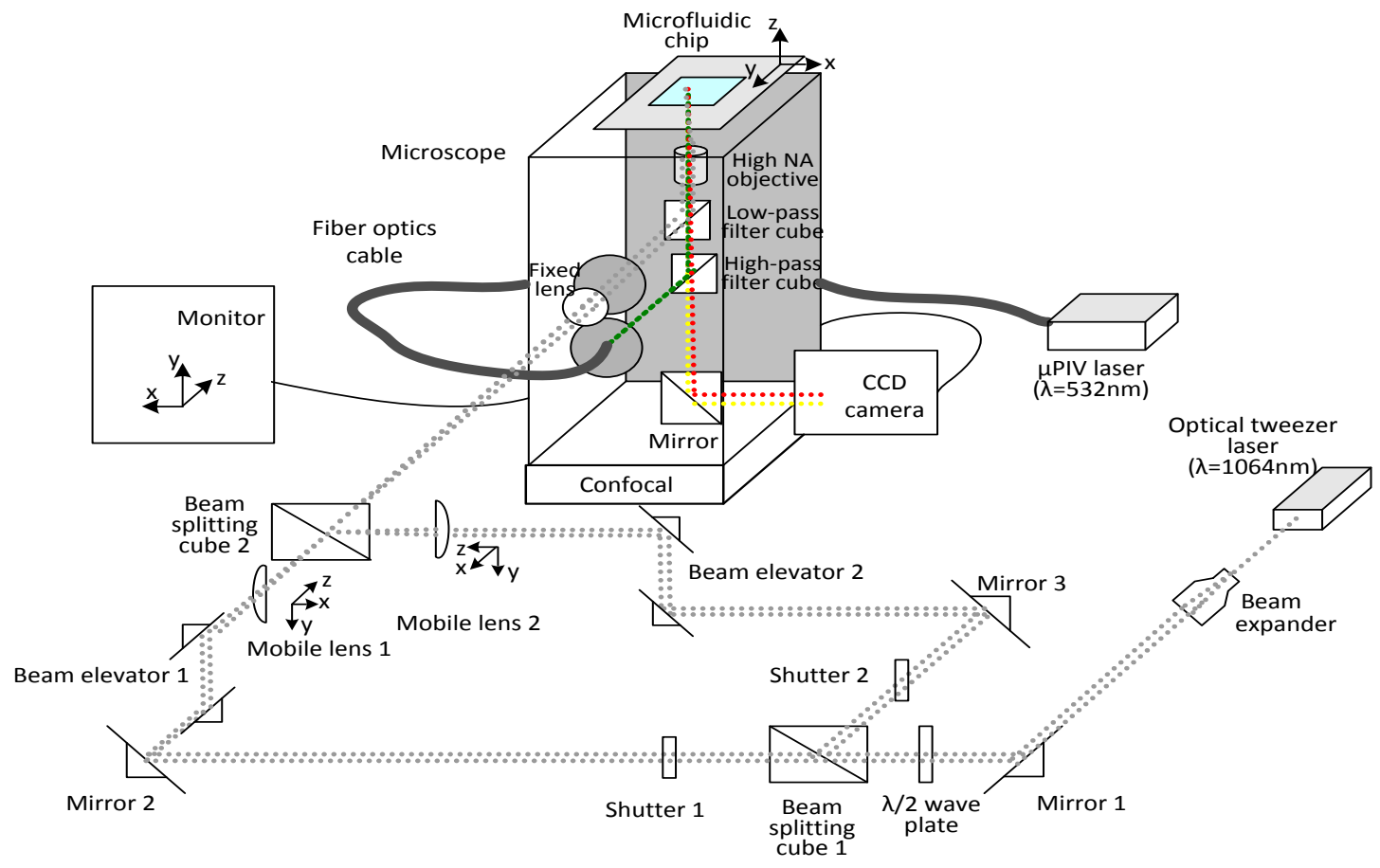

Figure 22: Schematic of the automated $\mu$ PIVOT device and the current optical arrangement. The OT laser goes through different optical components including beam splitting cubes to allow the existence of two lasers of opposite polarization. The position of their focus point can be controlled independently using two mobile lenses that translate in any direction with automated control ( $0.1 \mu \mathrm{m}$ resolution). They can both operate simultaneously or individually, using shutters placed in the laser beams paths. Both of the OT laser beams reunite through a second beam splitting cube before entering the microscope through an optical port, while the $\mu$ PIV laser enters it through a second port via an optical cable. 


\section{CHAPTER 4}

MPIVOT VALIDATION

The $\mu$ PIV and OT techniques have successfully been integrated as the $\mu$ PIVOT with the individual capabilities of each technique maintained and simultaneous application of both techniques enabled. A major concern in integrating the techniques was the influence the integration may have on each technique and the compatibility of the simultaneous applications. Initially the characteristics of each technique were assessed then the possible interference between OT and $\mu$ PIV was investigated by examining the degree of $\mu$ PIV nanoparticle trapping by the OT in a static environment and under uniform flow conditions.

\subsection{CHARACTERIZATION OF THE OPTICAL TWEEZERS}

\subsubsection{RANGE OF MOTION AT THE SAMPLE}

Once both of the traps are aligned, the beams exhibit a symmetrical pattern when reflecting off the coverslip and viewed through the GeviCam CCD camera (Figure 23). The two traps in Figure 23 (Trap 2 on the left and Trap 1 on the right) exhibit different patterns. This difference is due to their different polarization. However they retain the exact same power when the half-wave plate splits the one beam into two equal powered beams. 
Figure 23: Reflection of the lasers of trap1 and 2 on the coverslip. The dissimilar morphologies are due to the difference in their polarization.

The focus height is determined from the patterns concentrating to the smallest spot before disappearing, as the objective lens is moved upward and the focus moves deeper into the specimen (Figure 24). This focus height depends on the objective lens location and the mobile lens location. Therefore it can be different between trap 1 and trap 2 .

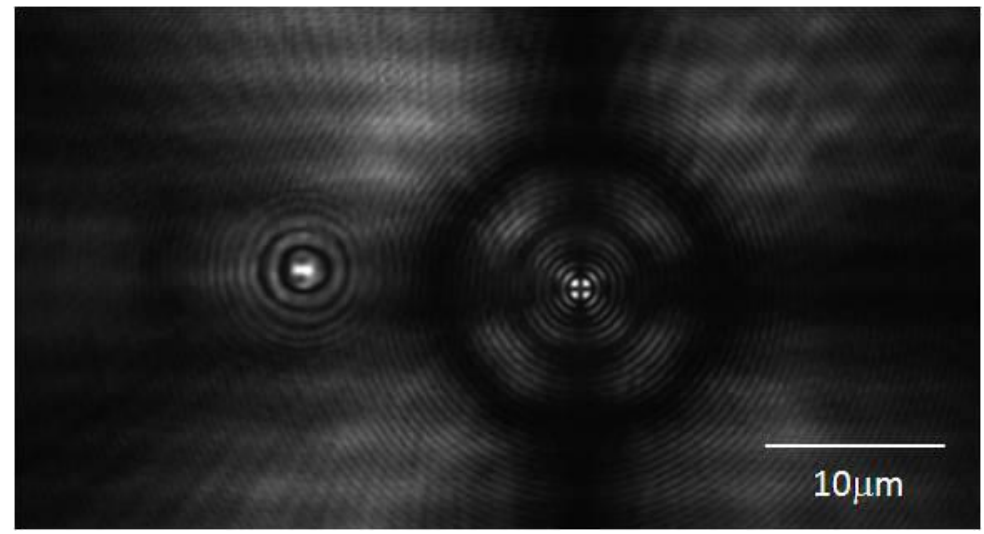

Figure 24: Trap 1 and 2 when in focus (in the sample, above the coverslip). 
It is observed for the 60x objective lens and the GeviCam camera (field of view of 60x80 $\mu \mathrm{m})$ that, in the image plane, a $0.0287 \mathrm{~mm}$ movement of the mobile lens corresponds to a $1 \mu \mathrm{m}$ movement. With the stage having a total range of $25 \mathrm{~mm}$, the possible horizontal movement at the sample is $865.8 \mu \mathrm{m}$, which is more than ten times the width of the field of view. The mobile lens home position is set at $6 \mathrm{~mm}$ from the edge. Therefore, the trap range is $207.8 \mu \mathrm{m}$ to the left and $658 \mu \mathrm{m}$ to the right. In the vertical direction ( $y$ direction in camera frame), a $0.0574 \mathrm{~mm}$ movement of the mobile lens corresponds to a $1 \mu \mathrm{m}$ movement. With the stage having a total range of $12.5 \mathrm{~mm}$, the possible vertical movement at the sample is $218.0 \mu \mathrm{m}$, which is more than three times the height of the field of view. Similar to the horizontal stage, the home position is set at $6 \mathrm{~mm}$ from the edge. Hence, the trap range is $104.6 \mu \mathrm{m}$ in the positive y direction and $113.4 \mu \mathrm{m}$ in the negative $\mathrm{y}$ direction.

For the calibration of the out of plane (z) direction, the measurements were more complex. A movement of the objective lens changes the height of the plane of focus, but also the height of the laser trap. The linearity with the movement in the $z$ direction of the mobile lens has however been performed by Chris Hinojosa, undergraduate at Portland State University, Mechanical Engineering. He found that in the out of plane direction ( $\mathrm{z}$ ) a $0.746 \mathrm{~mm}$ movement of the mobile lens corresponds to a $1 \mu \mathrm{m}$ movement at the sample. The perfect coincidence between the focus plane of the objective lens and the trap focus was set for the z-position of the mobile lens being at 
$15 \mathrm{~mm}$ (total range of $25 \mathrm{~mm}$ ) from the edge. Thus the possible movements relative to the focus plane are $15 \mathrm{~mm}$ (corresponding to $20.1 \mu \mathrm{m}$ at the sample) deeper into the specimen and $10 \mathrm{~mm}$ (corresponding to $13.4 \mu \mathrm{m}$ at the sample) towards the coverslip.

Figure 25 provides a schematic of the possible movements of the optical trap in all three directions at the sample.
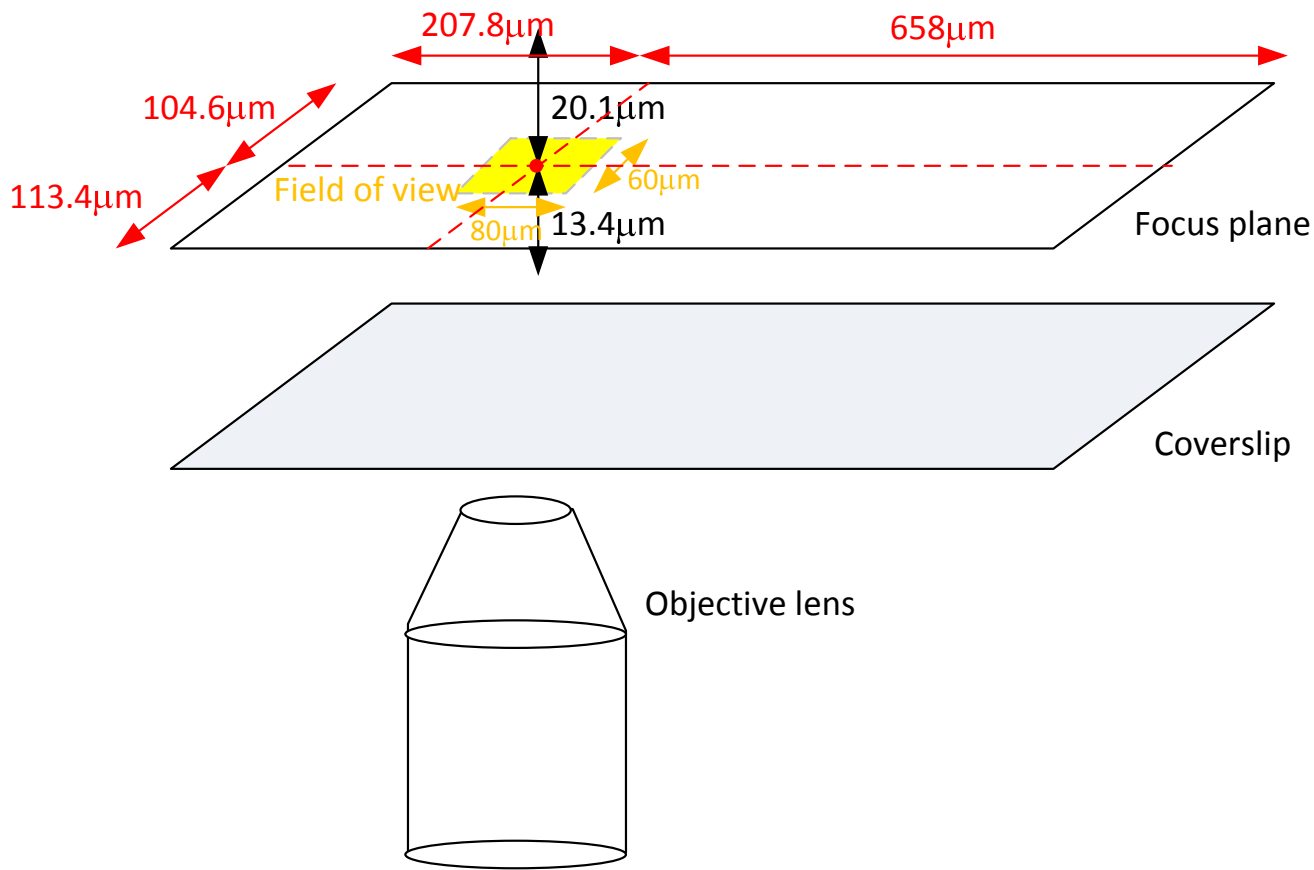

Figure 25: Schematic of the movement range of each trap at the sample in all three directions. The red spot indicates the mobile lens home position. In the focus plane, only a range equal to the field of view is necessary, but the possibilities of the instrumentation outrun this constraint. 


\subsubsection{OT LASER POWER}

The laser power was measured using a hand-held power meter (1916-C,

Newport) linked to a detector (818P-010-12, Newport). The digital results were accurate to $\pm 2 \mathrm{~mW}$. Figure 26 shows the different locations the power was measured within the system including just after the laser head $(\mathrm{H})$, after the first beam splitting cube for trap 1 (B1) and trap 2 (B2), before entering the microscope (M), in front of the objective lens (O), and at the sample (S).

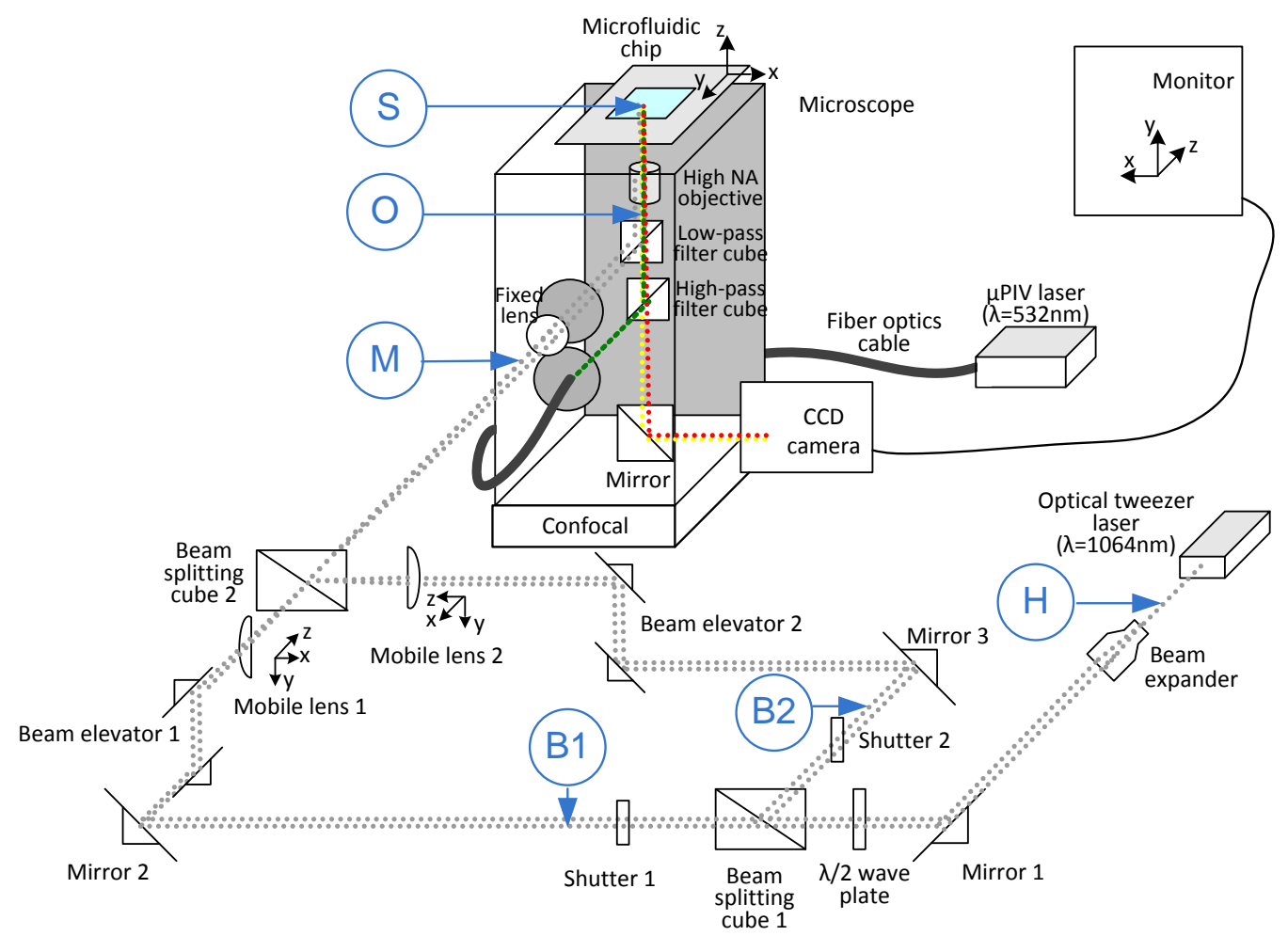

Figure 26: $\mu$ PIVOT system. The circled letters represent the different positions of power measurements: $\mathrm{H} \rightarrow$ laser Head, B1 and B2 $\rightarrow$ traps 1 and $\mathbf{2}$ after Beam splitting cube, $\mathrm{M} \rightarrow$ before entering the Microscope, $O \rightarrow$ before the Objective lens, $S \rightarrow$ at the Sample. 
The mostly accurate results are obtained when the beam diameter measured is centered on the $12 \mathrm{~mm}$ diameter sensor face and fills at least $50 \%$ of the sensor. The best power measurements were obtained in the optical path after the beam expander but before the objective lens. Figure 27 shows the laser power measured at the positions labeled in Figure 26 for different diode currents. Relative to the power at the laser head $(\mathrm{H})$, an average of $88 \pm 2 \%$ of the power was sustained before entering the microscope (M), $85 \pm 1 \%$ passed through the fixed lens and the low pass filter cube $(0)$, and $33 \pm 4 \%$ was transmitted to the sample (S).

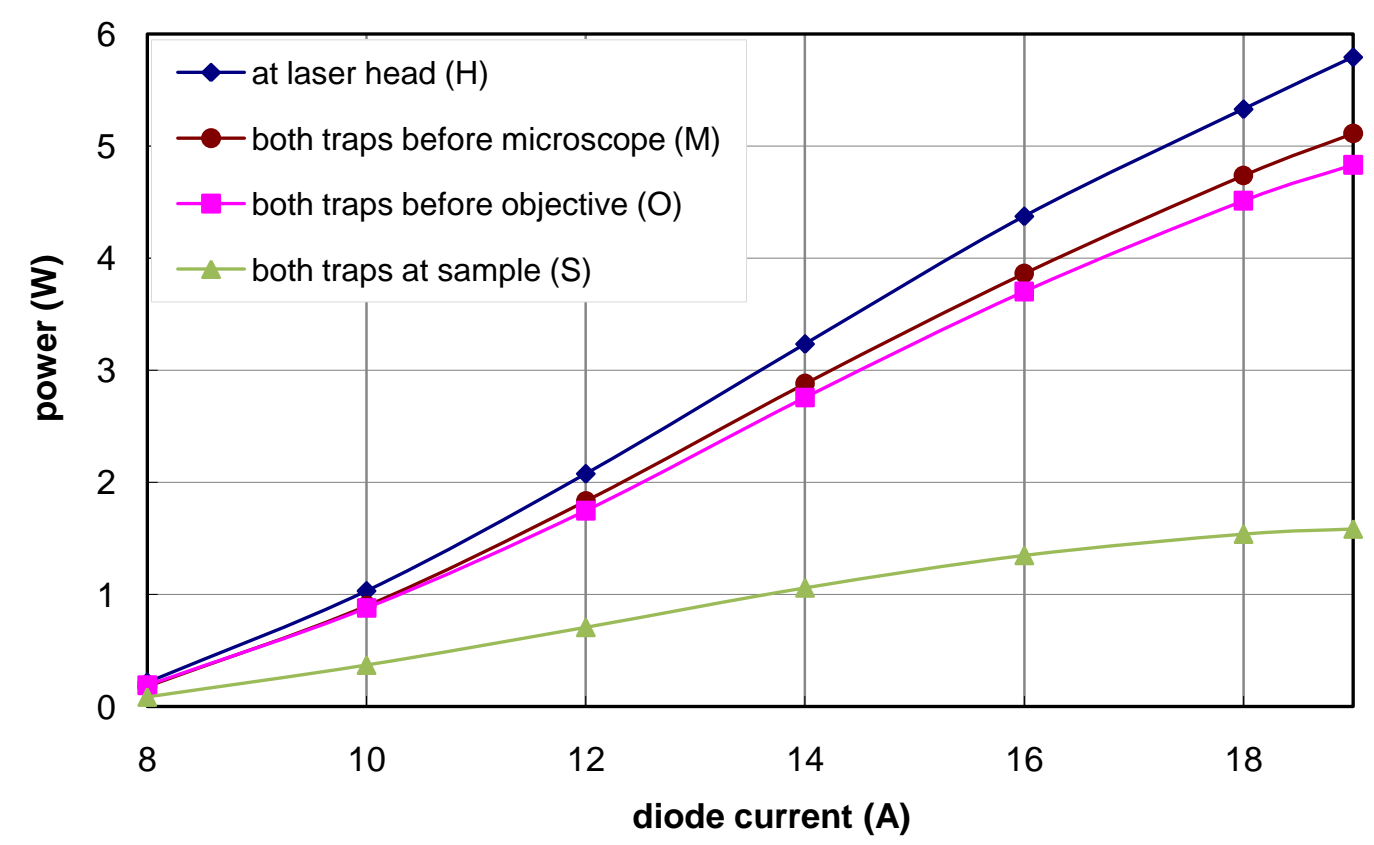

Figure 27: Variation of laser power with position when going through the optical path. The letters in parenthesis refer to Figure 26. 
Measurements at the laser head and after the objective lens are particularly inaccurate due to the $0.350 \mathrm{~mm}$ beam diameter at the laser head and the $1 \mu \mathrm{m}$ focal spot of the objective. This inaccuracy limited our ability to measure the light transmission through the objective lens. Using the dual objective method (see Svoboda and Block [Svoboda, 1994] for details) Neuman et al. [Neuman, 2004] measured the transmission efficiency for different objective lenses. For a Nikon oil-immersion 60x, N.A. 1.4 objective lens (the most similar to our objective lens) they measured a transmission of $39 \%$. By inserting the power meter before and after the objective lens, the transmission through our objective lens was measured at $30 \%$. Thus, the measured transmission may underestimate the actual transmission by approximately $30 \%$. Note, we only have one objective lens and are thus unable to verify our transmission with the dual objective method.

For consistency, measurement accuracy, and a conservative estimate of power at the sample, the laser powers in all experiments were measured after the first beam splitting cube for either trap 1 or trap 2. The power at the sample was estimated by combining the measured power with an expected transmission of $39 \%$. 


\subsubsection{Variation with half-wave plate position}

Figure 28 shows the power of each trap for different half wave plate angles. The diode current was set to the minimum $(8 \mathrm{~A})$ and the power was measured just after the first beam splitting cube.

As explained in Chapter 3, a perfect equal split of the laser should be obtained for a half-wave plate angle of $22.5^{\circ}$ from the vertical. This corresponds to an angle of $292.5^{0}(270+22.5)$. The results in Figure 28 seem to show a perfect split for a half-wave plate angle of $290^{\circ}$. This slight difference could be due to a small error in the half-wave plate positioning of the half-wave plate, or a small laser leak when the beam passes through the beam splitting cube. Regardless, the half-wave plate was set at $290^{\circ}$ for all experiments.

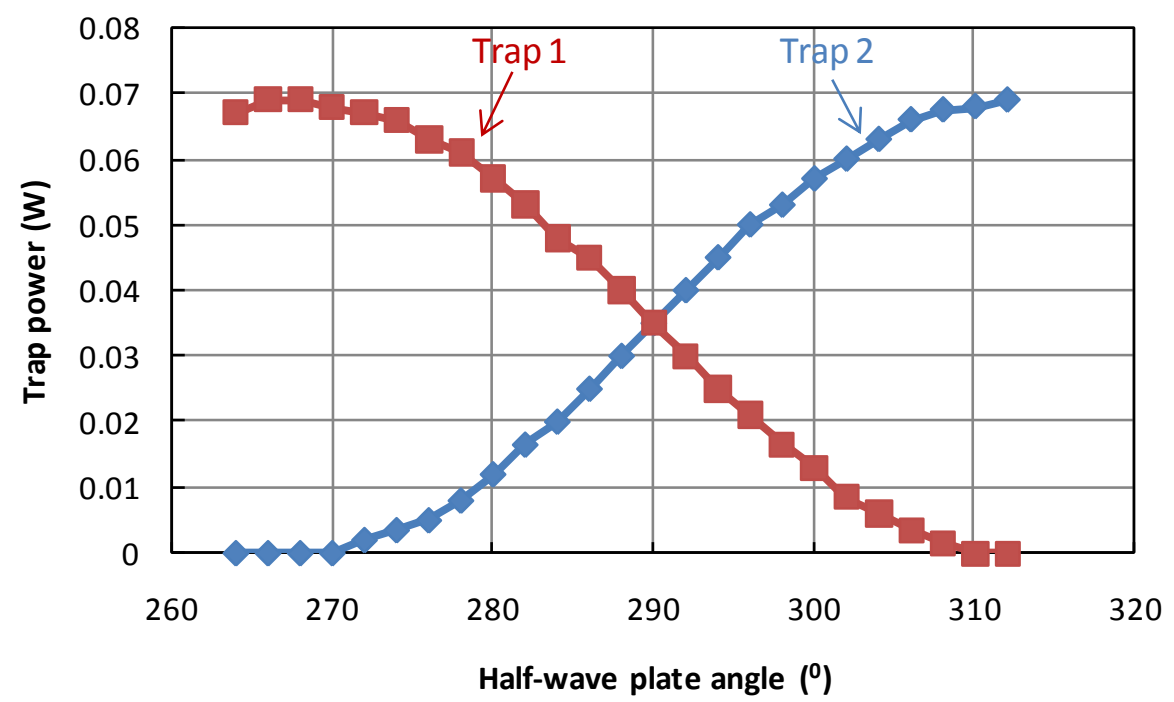

Figure 28: Trap power variation with half-wave plate position. 


\subsubsection{Variation with time}

Figure 29 shows the variation of laser power with time. The laser was fired at a constant diode current of $8 \mathrm{~A}$.

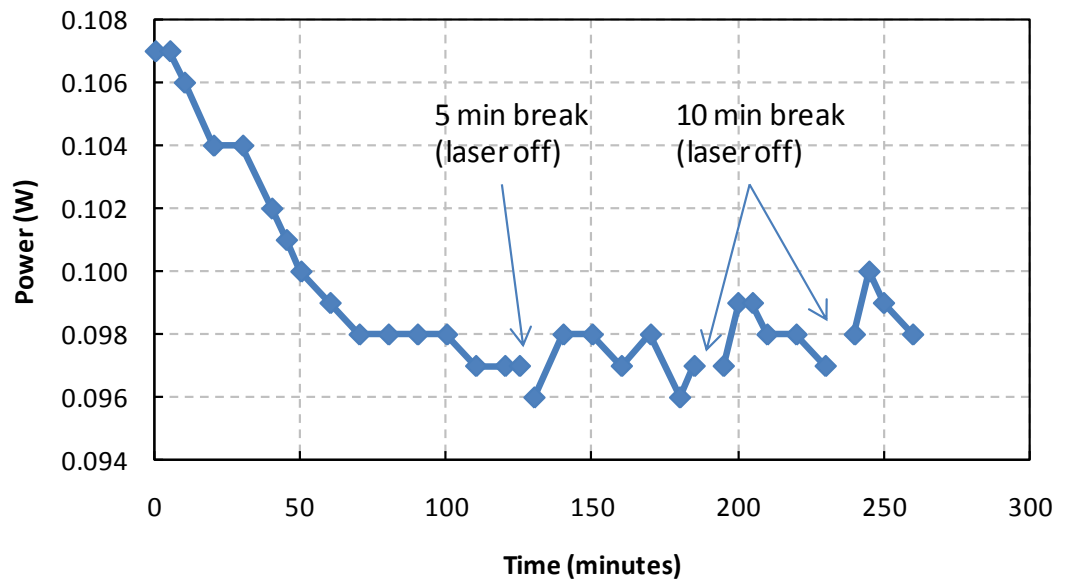

Figure 29: Trap 1 time dependence of the laser power measured after the first beam splitting cube.

The laser power decreases monotonically for the first 100 minutes attaining a steady state plateau. Note, the 5 minutes and 10 minutes breaks in Figure 29 were executed to determine the effects on laser power stability if small breaks during experiments for beam realignment or sample changes occurred. To verify the consistency of this phenomenon, Figure 30 shows time dependent measurements at other diode currents. The same general decrease for the first hour and a half before stabilization is observed. This extensive warmup time to stable laser power is surprising. As a result, all experiments are performed after at least 100 minutes of firing time to ensure a constant stable laser power. 

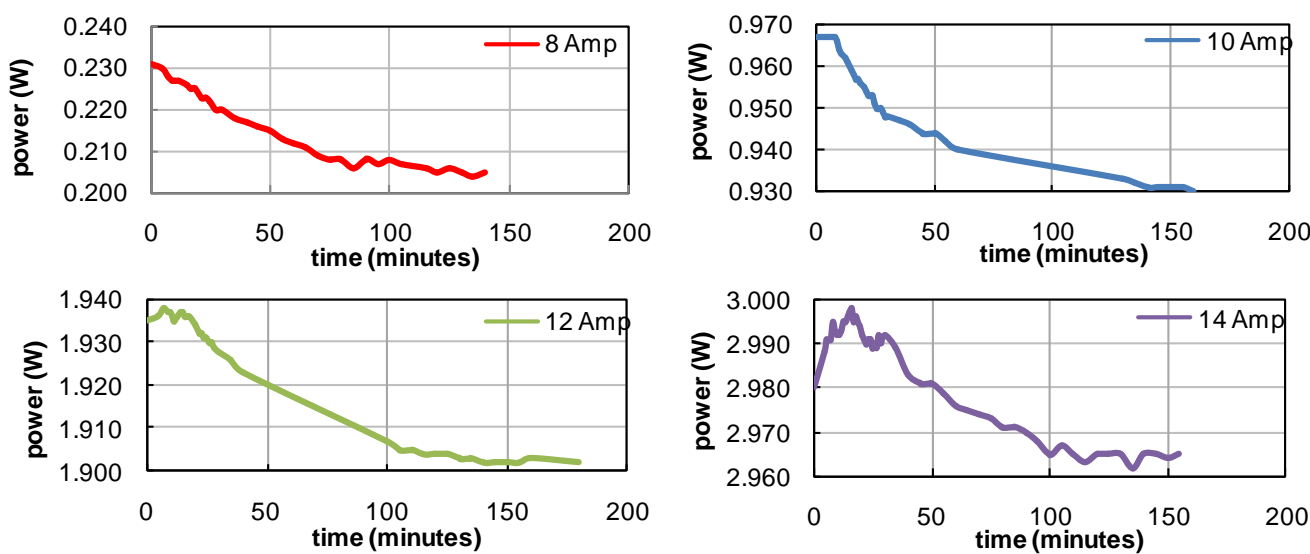

Figure 30: Power fluctuation with time for different diode currents. Measurements taken for both traps before entering the microscope (at M).

\subsubsection{OT ALONE: PERFORMANCE}

With dual optical tweezers, we have trapped and manipulated small glass microspheres (Soda lime glass, model 07666-1, Polysciences Inc., Warrington, PA), polystyrene microspheres, as well as skin, blood, bone, and cartilage cells (diameter range: 2 to $40 \mu \mathrm{m}$ ) in a static environment.

As preliminary studies, the trap stiffness was calculated (by the drag force method) for suspended microspheres (diameters between 20 and 23 microns) with laser power settings varying between $0.105 \mathrm{~W}$ and $1.481 \mathrm{~W}$. The fluid velocities were set such that the drag force was insufficient to displace the trapped particle outside the linear trap regime. Figure 31a shows the trap stiffness varying linearly between 4 and $70 \mathrm{pN} / \mu \mathrm{m}$ with laser power, while Figure $31 \mathrm{~b}$ shows the trap stiffness decreasing towards a plateau with increasing particle diameter. 

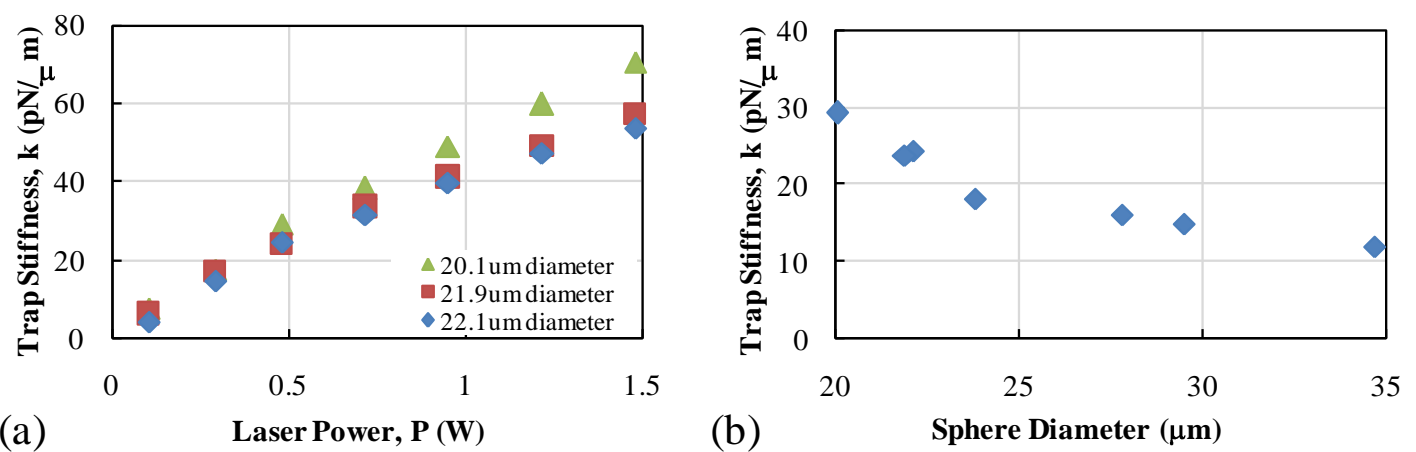

Figure 31: (a) Trap stiffness as a function of the applied laser power for trapped polystyrene microspheres. (b) Trap stiffness as a function of sphere diameter for a constant laser power of $0.48 \mathrm{~W}$. Stiffness values were determined from linear curve fits of the drag force versus microsphere displacement experimental data. Drag force calculations accounted for channel wall proximity.

This behavior is consistent with previously reported stiffness values [Williams, 2002].

This indicates that the integration of the OT and the $\mu$ PIV laser techniques, which involved the placement of an additional filter cube in the OT path, did not interfere with the capabilities of the OT system.

\section{2 $\mu$ PIV CHARACTERISTICS}

With $\mu$ PIV, two modifications possibly altering the image quality are the addition of a filter cube in the laser path, and the simultaneous firing of the OT laser.

Measurements of fluid velocities in custom microfluidic devices were achieved using the $\mu$ PIVOT device. Figure 32 shows a 2-D velocity field adjacent to the side wall at the midplane of a microchannel with a velocity vector vertical spacing of $436 \mathrm{~nm}$. 


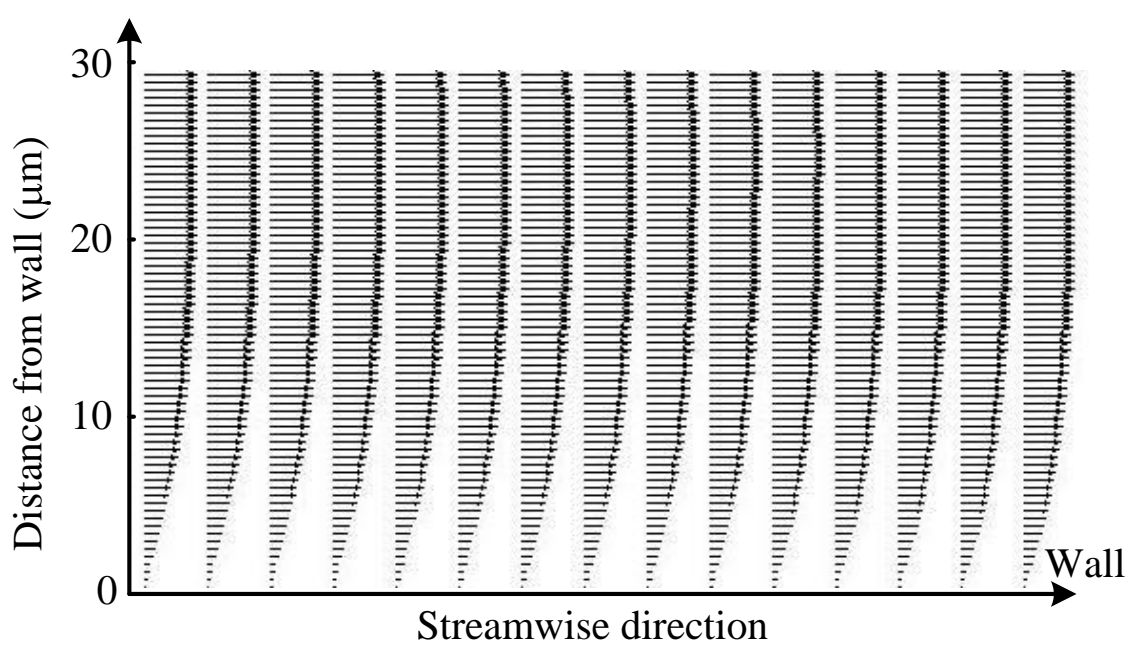

Figure 32: Laminar flow velocity profiles acquired with $\mu$ PIV. The near wall velocity resolution is $436 \mathrm{~nm}$. The no-slip condition can be observed as the velocity decreases (vector length $=$ magnitude) to 0 at the wall.

The velocity approaches its free-stream value at approximately $20 \mu \mathrm{m}$ (y-direction) and smoothly decreases to zero at the wall. This profile is consistent with the analytical solution for flow through a rectangular duct assuming the no-slip boundary condition [White, 1974], and concurs with previous studies performed with $\mu$ PIV [Tretheway, 2002]. This indicates that the addition of optical components, specifically the low pass filter necessary for the set up of the integrated device, does not decrease the capabilities of the $\mu$ PIV.

\subsection{INTERACTIONS BETWEEN OT AND $\mu$ PIV}

The $\mu$ PIV lasers volume illuminate the entire field of view during two, $5 \mathrm{~ns}$ pulses. As expected, due to the short and diffuse nature of this lighting, no apparent effect of the $\mu$ PIV lasers on a particle trapped with OT was observed. The particle remained 
stationary and in focus during each pulse even at the maximum pulse energy of $50 \mathrm{~mJ}$. During $\mu$ PIV measurements, the laser power is reduced to the minimum power (less than $25 \mathrm{~mJ}$ per pulse) necessary to excite the fluorescent nanoparticles.

A greater concern was the effect of the OT on the $\mu$ PIV nanoparticles. The fluorescent nanoparticles are much smaller than the IR wavelength of the OT laser, however, the influence of the OT on the nanoparticles is not known. In order to successfully apply both techniques simultaneously, the influence of the OT on the flow trajectory of the $\mu$ PIV nanoparticles ( $275 \mathrm{~nm}$ diameter) must be determined.

Figure 33a shows the effect of the OT laser power on a stagnant solution of $\mu$ PIV nanoparticles while Figure $33 \mathrm{~b}$ shows the $\mu \mathrm{PIV}$ solution moving at a constant velocity set with the automated translation stage (streaklines indicate the direction of flow). The solution contained a typical concentration of $\mu \mathrm{PIV}$ nanoparticles (concentration of $50 \mu \mathrm{L}$ nanoparticle, $2 \%$ solid solution for $2 \mathrm{~mL}$ of distilled water). In each case, the OT laser was off then turned on at a given power. For all laser powers, a small region of trapped nanoparticles is observed. These trapped nanoparticles fluorescence and appear as bright spot (note: due to the resolution of Figure 33, the fluorescent spot at $0.11 \mathrm{~W}$ may be difficult to discern). 
(a)

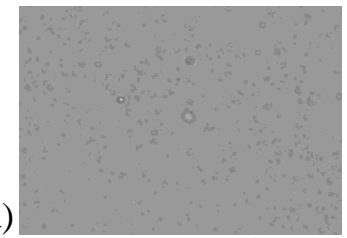

$0.11 \mathrm{~W}$

(b)

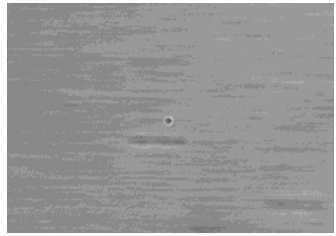

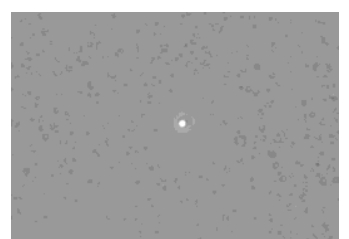

$0.49 \mathrm{~W}$

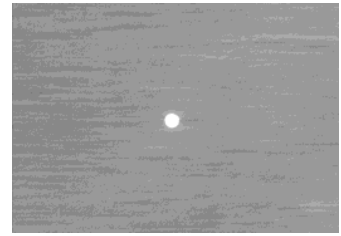

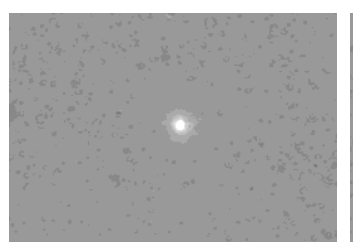

$0.95 \mathrm{~W}$

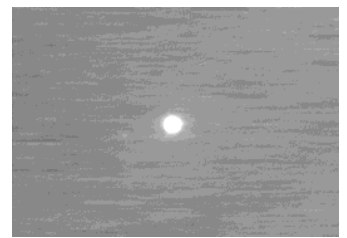

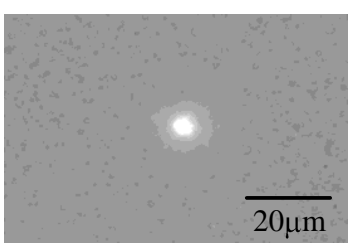

$1.45 \mathrm{~W}$

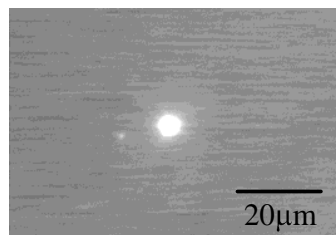

Figure 33: Response of $\mu$ PIV nanoparticles to the applied trap laser power (measured at the microscope entrance). The number of trapped nanoparticles (bright spot) increases with trap power within (a) a static fluid and (b) a fluid moving at constant velocity (shown by the streaklines).

This trapped cluster of particles exhibits a dynamic growth (accumulation of particles) and collapse (release of particles) behavior. Even with this dynamic clustering, no attraction of nanoparticles to the OT in the field of view is observed for either the static solution or moving fluid. The streaklines in Figure 33b are straight throughout the field of view even near the fluorescent spot, which indicates a localized region of influence.

This is further verified by calculating the fluid velocity with $\mu$ PIV. The measured velocities are within $1 \%$ of the translation stage velocity except in the region of influence where velocities can not be calculated due to pixel saturation in the $\mu$ PIV images. This suggests the dynamic behavior occurs along the optical axis. The maximum region of influence (as shown in Figure 33) increases from a measured diameter of $0.7 \mu \mathrm{m}$ for $0.11 \mathrm{~W}$ to a diameter of $4.0 \mu \mathrm{m}$ for $1.45 \mathrm{~W}$. While the actual size of the nanoparticle cluster can not be definitively determined, the nanoparticle cluster 
is within the region of influence. Thus, this region of influence represents the upper limit of nanoparticle cluster size.

It is interesting to note that Figure 33 shows the $\mu$ PIV nanoparticles fluorescing within the region of influence in each image without applying the required excitation wavelength of $532 \mathrm{~nm}$. This is most likely due to two photon excitation from the trapping laser light [Florin, 1996][Florin, 1997]. While this phenomenon interferes with $\mu$ PIV by saturating pixels as stated above, the phenomena is limited to the localized region of influence.

\subsection{SUMMARY}

The $\mu$ PIVOT maintains the individual capabilities of $\mu$ PIV and OT. Without a trapped micron-sized object, a localized dynamic nanoparticle accumulation and release in the vicinity of the OT is observed. Outside this region of interest, the clustering process has no measurable effect on velocity measurements in the image plane. This indicates the dynamic process occurs along the optical axis (out of the image plane). As the out of plane extent is unknown, it is not clear, at this time, how significant this effect would be on 3-D velocity measurements. For measurements with a suspended sphere in the image plane, the clustering process is generally negligible. An array of single sphere experiments is discussed further in the next chapter. 


\section{CHAPTER 5}

FLOW AROUND A SINGLE FREELY SUSPENDED SPHERE

The objective of the experiments reported in this chapter is to create a controlled and measured constant flow around a stationary particle not attached to any surface or mechanical restraint. The experiments are unique and can be verified with theoretical and computational models. Three types of flow were examined: 1) a gravity driven flow through a straight microchannel, 2) a uniform flow field generated by moving the fluid sample with an automated translation stage, and 3) a gravity driven planar extensional flow. To push the validation further, the trap stiffness of our optical trap was measured for different sphere sizes at different depths into the sample. For this study, the uniform flow was used to apply a constant and known force (the drag force) on the microsphere. These results completed the validation of the $\mu$ PIVOT.

\subsection{EXPERIMENTAL PROTOCOL}

\subsubsection{STATIONARY SPHERE IN A GRAVITY DRIVEN MICROCHANNEL FLOW}

Figure 34a provides a schematic of a spherical particle trapped by the optical trap (OT) in a 50x500 $\mu \mathrm{m}$ microchannel. 


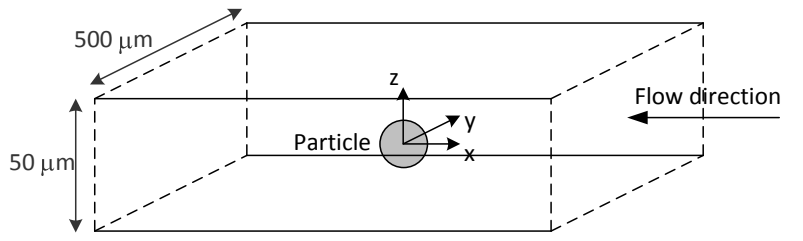

(a)

Channel

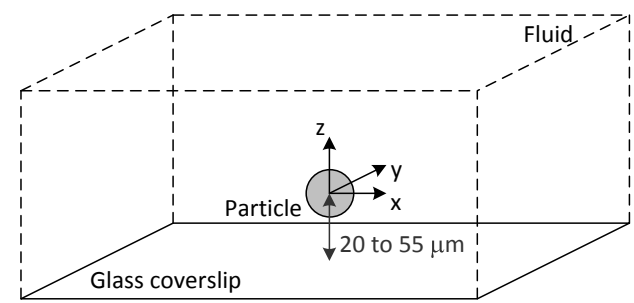

(b)

Stage velocity

Figure 34: A 3-D simplified sketch of the single sphere in flow experiment. (a) A particle trapped in the middle of a straight channel with laminar flow (Reynolds number $=10^{-2}$ ). (b) A sphere held stationary in between two coverslips moving at a constant velocity. The sphere is more than $1 \mathrm{~mm}$ below the top coverslip.

This straight channel was fabricated using a standard soft lithography approach [Xia, 1998]. Molds on silicon wafers were ordered to the Stanford Microfluidics Foundry, Stanford University, CA, USA using custom-designed AutoCAD (San Rafael, CA) drawings. For the experiments stated in this chapter, simple straight channels were designed. Chips were fabricated with polydimethylsiloxane (PDMS) (Sylgard 184, Dow Corning, Midland, MI) as the soft peeled material. The thickness of the PDMS was $0.5 \mathrm{~cm}$ to $1 \mathrm{~cm}$ to insure enough stiffness and negligible deformation of the channels. Holes were punched in the PDMS to allow flow through the channels once bonded (Figure 35).

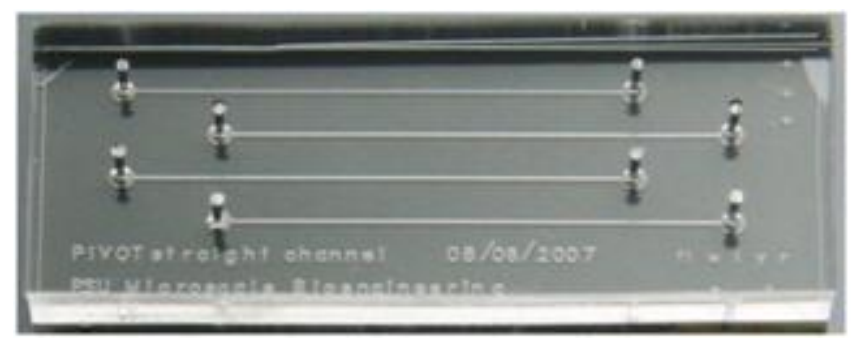

Figure 35: A PDMS chip bonded to a glass coverslip with four 50x500 $\mu \mathrm{m}$ straight channels. The experiments were conducted in a single channel. 
The chip was bonded to a coverslip by two possible ways: plasma bonding at the Stanford Microfluidics Foundry (irreversible), or chemical bonding in our laboratory (reversible) using the PDMS Replica Molding and Microfluidic Device Finishing protocol [Pang, 2006].

A gravity driven microchannel flow was generated by simply attaching input and output open syringes (filled barrel with no plunger) placed at different heights. The output syringe height was held constant while the input syringe height was controlled in order to vary the flow rate. The syringes were connected to the microfluidic chip via Tygon tubing (Fisher Scientific, Pittsburg, PA) attached to metal pins (0.025 ODx0.017 ID, Type 304, New England Small Tubes Corp., Litchfield, NH) that were directly inserted at the entry/exit ports of the microchannel through the punched holes. This setup allowed for a controllable, constant, and steady flow.

The microfluidic chip was initially primed with particle free de-ionized water. A low concentration of polystyrene microspheres solution with an average diameter of 26 $\mu \mathrm{m}$, diluted in distilled water at a volume ratio of 1:1000, was then inserted through the upstream tubing. A single microsphere was trapped far from the entry/exit ports in order to be in a fully developed flow region, and the remaining microspheres were flushed out of the microchannel. All the experiments were performed at $z=0$ (center of the channel), calculated by locating the top and bottom of the channel then fine tuning 
the microsphere position by observing its rotation. No rotation corresponds to symmetry in the velocity field experienced by the sphere. This occurs only at the midplane between the two walls. The gravity driven microchannel flow was then turned on. This fluid was seeded with fluorescent nanoparticles for $\mu$ PIV measurements around the microsphere. The captured $\mu$ PIV images were examined with an initial interrogation window of $64 \times 64$ pixels and $75 \%$ image overlap, then further refined to $32 \times 32$ pixels (a common $\mu$ PIV interrogation domain) with a $50 \%$ overlap (yielding a $1.7 \mu$ m velocity spacing). This resolution is sufficient for the particle sizes examined. The time difference between images $(\Delta \mathrm{t})$ ranged from $500 \mu$ s to $2 \mathrm{~ms}$ for a 6-10 pixel movement between image frames. This setting provides optimum image quality for the velocity vectors analysis.

\subsubsection{STATIONARY SPHERE IN A UNIFORM FLOW}

Figure $34 \mathrm{~b}$ provides a schematic of a spherical particle trapped by OT in between two coverslips. A very low concentration of polystyrene microspheres solution (Polystyrene crosslinked DVB copolymer, Duke Scientific, Fremont, CA), with diameters ranging from 10 to $35 \mu \mathrm{m}$ (sizes of biological cells), was diluted in a solution of typical concentration fluorescent nanoparticles (for $\mu$ PIV measurements around the suspended microsphere) and placed between two coverslips. A sphere was trapped at a depth of 1.5 times the sphere diameter (between 20 and $55 \mu \mathrm{m}$ ) from the bottom coverslip to maintain a constant dimensionless distance (depth/sphere diameter) from the bottom 
coverslip. The distance between the focus plane and the coverslip $\left(h_{\text {focus }}\right)$ was set with the objective lens of the microscope (vertical resolution of $250 \mathrm{~nm}$ ). Taking into account the refractive index mismatch between the coverslip $\left(n_{\text {glass }}\right)$ and the flow medium $\left(\mathrm{n}_{\text {water }}\right)$, the actual depth of the trapped sphere $\left(\mathrm{h}_{\text {sphere }}\right)$ was calculated as [Vermeulen, 2006]:

$$
\mathrm{h}_{\text {sphere }}=\left(\mathrm{n}_{\text {water }} / \mathrm{n}_{\text {glass }}\right) \cdot \mathrm{h}_{\text {focus }}
$$

The upper coverslip is greater than $1 \mathrm{~mm}$ from the suspended sphere and thus does not affect the trapped sphere hydrodynamically [Ho, 1974]. The uniform flow field was generated by moving the reservoir (made by the two coverslips) on the microscope automated stage at constant velocity. The stage was controlled to execute displacement rates of $50 \mu \mathrm{m} / \mathrm{s}$ to $500 \mu \mathrm{m} / \mathrm{s}$ in the $\mathrm{x}$ direction (linear resolution of 20 $\mathrm{nm})$.

The captured $\mu$ PIV images were examined as described in the section 5.1.1. The time difference between images $(\Delta t)$ was set to be from 2.5 ms (for a stage velocity of $500 \mu \mathrm{m} / \mathrm{s}$ ) up to $20 \mathrm{~ms}$ (for a stage velocity of $50 \mu \mathrm{m} / \mathrm{s}$ ) so that the typical movement of the nanoparticles was approximately 10 pixels between image frames. 


\subsubsection{STATIONARY SPHERE IN AN EXTENSIONAL FLOW}

Figure 36 provides a schematic of a spherical particle trapped by the OT in a 50×500 $\mu \mathrm{m}$ cross-junction. The flow generated by this geometry is a pseudo-planar extensional flow. At the center of this cross-junction there is a stagnation point where a symmetric particle would experience no net drag. This cross-junction design was fabricated with the soft lithography technique. Figure 37 shows the mold (a) and the microfluidic chip (b) of the channel.

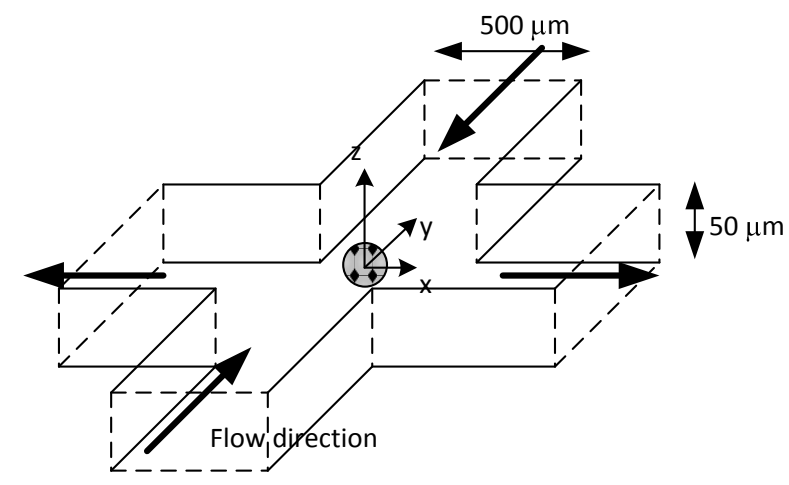

Figure 36: Schematic of a sphere trapped at the stagnation point of a cross-junctional flow. The bold arrows represent the flow directions.
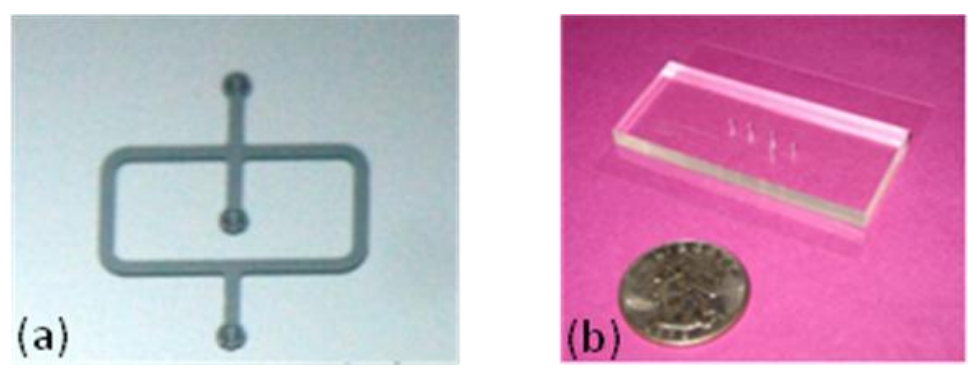

Figure 37:(a) Cross-junction silicone mold. (b) Microfluidic chip. 
The microfluidic chip containing the cross-junction geometry was initially primed with particle free de-ionized water. A low concentration of polystyrene microsphere solution with an average diameter of $26 \mu \mathrm{m}$, diluted in distilled water at a volume ratio of 1:1000, was then inserted through the upstream tubing. Using a very slow flow (less than $50 \mu \mathrm{m} / \mathrm{s}$ ) a single microsphere was trapped in the region close to the stagnation point and slowly brought to the stagnation point. To ensure the sphere was positioned right at that point, the trap was shuttered for an instant. Depending on where the sphere would migrate, we could infer the position of the sphere related to the stagnation point. The trapped sphere was therefore put in position by small iterations. Once in place, the remaining microspheres were flushed out of the microchannel. All the experiments were performed at $z=0$ (center of the channel), calculated the same way as described in straight microchannels. The gravity driven microchannel flow was then turned on. This fluid was seeded with fluorescent nanoparticles for $\mu$ PIV measurements around the microsphere. Here again, the captured $\mu$ PIV images were examined with an initial interrogation window of $64 \times 64$ pixels and $75 \%$ image overlap, then further refined to $32 \times 32$ pixels. The time difference between images $(\Delta t)$ was set to $3 \mathrm{~ms}$ for a 5-10 pixel movement between image frames at the region of interest (approximately one radius away from the sphere surface). This setting provides optimum image quality for the velocity vectors analysis. 


\subsubsection{TRAP STIFFNESS MEASUREMENTS}

The trap stiffness is an important parameter of the optical trap (OT). It depends on the objective's numerical aperture, the laser's wavelength, the power of the laser, its polarization and beam profile, the refractive indices of the particle and the medium, and the particle's size and shape. As described in the Optical Tweezers review (Section 2.3), an object trapped in an OT is not completely immobile. The OT has a stiffness which prevents the object from escaping when subjected to other forces than the OT force. That is, the OT is a potential well, with a stiffness $k$. Just like a spring, this stiffness is the factor by which the force is linearly proportional to the distance that the trapped object is displaced from its equilibrium position. In our experiments, the force exerted on a trapped microsphere is the drag force, due to a uniform flow.

$$
F_{d r a g}=k . \Delta x
$$

$F_{d r a g}$ is the drag force, $k$ is the trap stiffness, and $\Delta x$ is the difference between the particle position when the particle is trapped without flow and trapped with flow (Figure 38). 

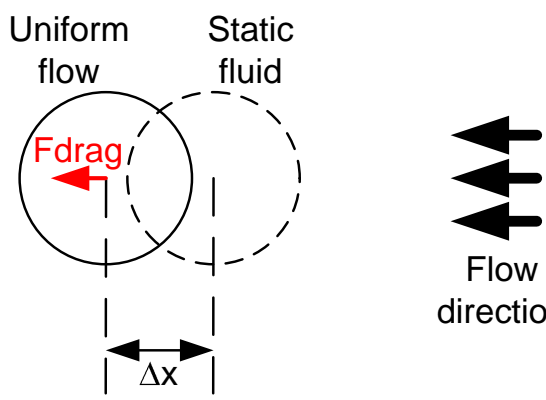

Flow direction

Figure 38: Schematic of trapped sphere displaced from its equilibrium position without and with uniform flow.

This sphere position was determined by taking pictures with the GeviCam CCD camera for the stage immobile or moving at a constant velocity. Captured images were further analyzed with an object tracking software (Spotlight-8, NASA) for locating position and size of trapped particles with a resolution of $+/-0.5$ pixels (Figure 39 ).

Note: the trap stiffness is more precisely the lateral (or radial) trap stiffness. We are focusing on the stiffness for lateral movements relative to the beam direction. The axial stiffness also exists and actually depends on the lateral stiffness [Sato, 1991].
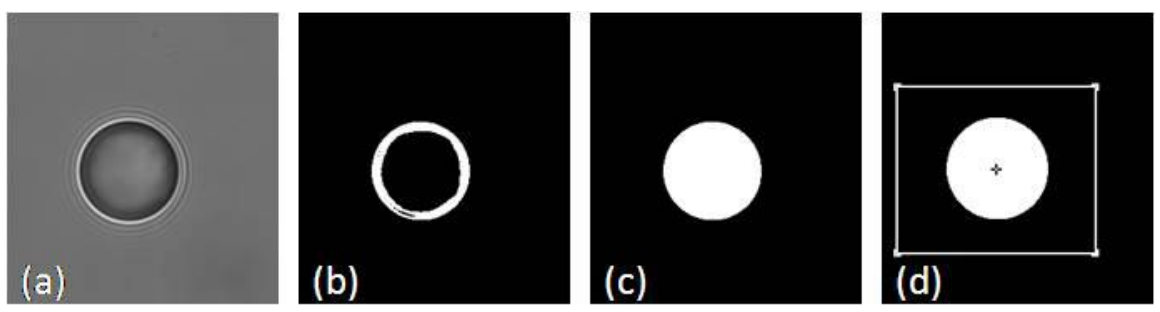

Figure 39: Basic steps for sphere position determination using the software Spotlight-8. (a) Raw image. (b) Standard threshold to obtain a white continuous border around the sphere. (c) Morphological hole fill, to obtain a black and white image of the sphere. (d) Center tracking to determine the center of the white object. 
Its determination is more complex and requires additional experiments that were not performed in this project.

The trap stiffness is calculated by linearly fitting a range of drag force $\left(F_{\text {drag }}\right)$ versus displacement data $(\Delta x)$. Using the same trapped sphere and the same OT laser (power, intensity) at the same sample location (in plane and in depth), we apply several flow velocities. For a spherical object in a uniform flow near a wall, the drag force is directly proportional to the flow velocity. Knowing the distance from the wall, the drag force is calculated using Faxen's law [Happel, 1983]:

$$
F_{\text {drag }}=\frac{6 \pi \mu a v}{1-\frac{9}{16}\left(\frac{a}{l}\right)+\frac{1}{8}\left(\frac{a}{l}\right)^{3}-\frac{45}{256}\left(\frac{a}{l}\right)^{4}-\frac{1}{16}\left(\frac{a}{l}\right)^{5}}
$$

where $a$ is the radius of the spherical particle, $l$ is the distance from the wall, $\mu$ is the fluid viscosity, and $v$ is the fluid velocity experienced by the sphere. This modified Stokes drag accounts for particle-wall effects associated with the presence of the coverslip.

The velocities imposed on the flow for different sphere radii are:

- For $a<5 \mu \mathrm{m}$, the flow velocities were set to 0, 50, 100, 150, 200 and $300 \mu \mathrm{m} / \mathrm{s}$.

- For $a \geq 5 \mu \mathrm{m}$, velocities were set to $0,20,40,60,80,100,125,150$ and $175 \mu \mathrm{m} / \mathrm{s}$. 
Finally, the trap stiffness is evaluated taking into account only the linear portion of the $F_{\text {drag }}$ versus $\Delta x$ curve.

Two main reasons drove us to study the calculation of the trap stiffness. First, the measurement provides crucial information on the characteristics of our system and $\mathrm{k}$ is a well known important parameter for simple polystyrene microspheres. Second, the trap properties for microspheres serves as a benchmark for biological cell experiments (see next chapter).

\subsubsection{COMPUTATIONAL MODEL}

\subsubsection{Stationary sphere in uniform flow}

The flow around a sphere suspended in a uniform velocity field was modeled with computational fluid dynamics software (STAR-Design 4.14.003, CD-adapco, Melville, NY, for model and meshing, and STAR-CCM+2.10.017, for the computation). For the experimental conditions (microscale, low velocities), the flow is laminar $(\operatorname{Re}<3)$ and steady. The fluid was modeled as an incompressible Newtonian fluid with a density $\left(998 \mathrm{~kg} / \mathrm{m}^{3}\right)$ and viscosity $\left(0.992 \times 10^{-3} \mathrm{~N} . \mathrm{s} / \mathrm{m}^{2}\right)$ equal to that of water at room temperature $\left(20.5^{\circ} \mathrm{C}\right)$. Following the described experimental setup, a sphere was set to be $20 \mu \mathrm{m}$ in diameter and placed $30 \mu \mathrm{m}$ from the bottom coverslip. The bottom coverslip is modeled as a no-slip boundary moving at a constant velocity of $200 \mu \mathrm{m} / \mathrm{s}$ while a symmetry plane replaces the remaining computational boundaries including the 
top coverslip (there is no hydrodynamic interaction with the sphere) to reduce computational effort. The computational domain, shown in Figure 40a, was meshed with 134,375 polyhedrals to provide a numerical resolution greater than experimental measurements. We extracted velocities along the inflow/outflow axis (x-direction).

The solver uses the numerical 'SIMPLE' algorithm [Patankar, 1980] that iteratively solves the coupled Navier-Stokes equation and the mass continuity equation:

$$
\begin{aligned}
& \rho\left(\frac{\partial v}{\partial t}+\boldsymbol{v} * \nabla \boldsymbol{v}\right)=-\nabla p+\mu \nabla^{2} \boldsymbol{v}+\mathbf{f} \\
& \nabla * \boldsymbol{v}=0
\end{aligned}
$$

where $\rho$ is the fluid density, $v$ is the fluid velocity vector, $t$ is time, $\nabla p$ is the pressure gradient, $\mu$ is the fluid viscosity, and $\mathbf{f}$ is an added vector representing other forces applied to the fluid.

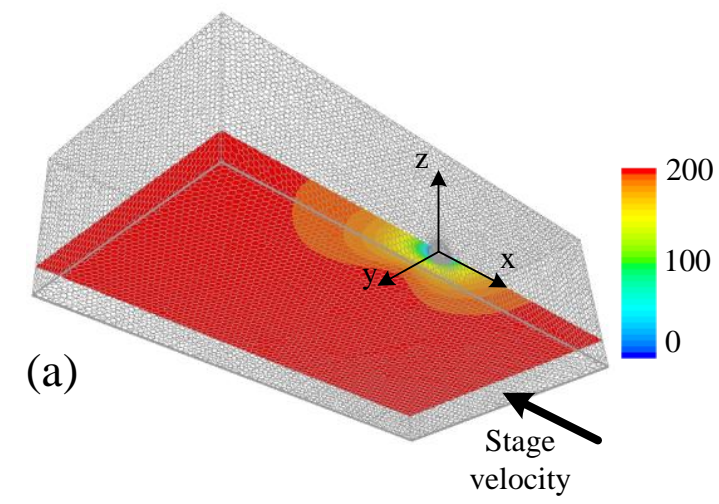

(b)

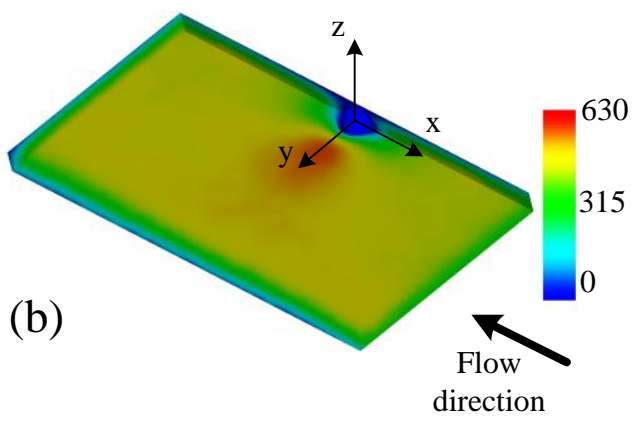

Figure 40: A 3-D representation of the computational flow domain. (a) Uniform flow half-space (b) Gravity driven flow quadrant. The color scales represent the magnitude of the velocity in the general flow direction, in $\mu \mathrm{m} / \mathrm{s}$ ( $x$ axis). 


\subsubsection{Stationary sphere in gravity driven microchannel flow}

In the second experimental setup, the width of the microchannel $(500 \mu \mathrm{m})$ is much larger than the height of the microchannel $(50 \mu \mathrm{m})$. Under these conditions, the flow at the center of the microchannel resembles Poiseuille flow between two infinite stationary parallel plates which was confirmed experimentally. However, the theoretical solution for Poiseuille flow around a suspended sphere is relatively complex with solutions limited to spheres with diameters sufficiently smaller than the plate separation [Ho, 1974], [Ganatos, 1980]. These solutions are not applicable to the single sphere experiments described here. As a result, the flow around a sphere suspended in a microchannel was modeled with computational fluid dynamics software (OpenFOAM, with the SimpleFOAM solver, OpenCFD, Berkshire, UK).

The solver uses the same numerical 'SIMPLE' algorithm described in the previous section and the fluid was also modeled similarly. The boundary conditions were the no slip condition at the channel walls and at the sphere surface. In addition, the simulation required an inlet velocity. The computational domain is sufficiently large that effects of the sphere at the inlet are negligible and the velocity field is consistent with flow through a microchannel without a sphere. The velocity for flow in the microchannel without a sphere (at the same conditions of the sphere experiment) was measured experimentally with $\mu$ PIV and the average input as the inlet velocity. To reduce computational effort, the flow domain was subdivided into quadrants (dictated by flow 
symmetry) and solved with 5,000+ tetrahedron elements for a single quadrant. Figure $40 \mathrm{~b}$ provides the quadrant computation domain and shows representative flow characteristics of this scenario. From this 3-D model, we examine the 2-D velocity profile at the mid-plane of the channel, coincident with the center of the microsphere (x-y plane in Figure 34a) which corresponds to the experimentally analyzed region. We also extract velocities along the inflow/outflow axis (x-direction).

\subsection{RESULTS}

\subsubsection{TRAPPED SPHERE IN A STAGNANT FLUID}

Figure 41 shows a large microsphere (diameter of $26 \mu \mathrm{m}$ ) trapped in a stagnant solution of nanoparticles.

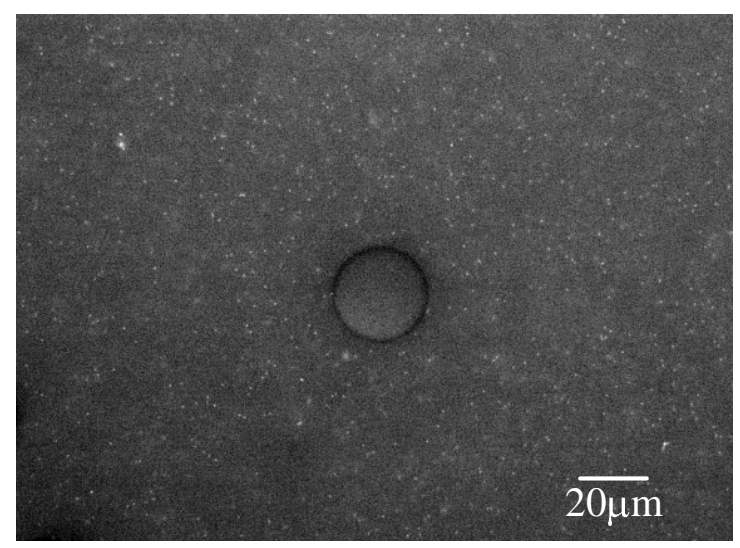

Figure 41: A trapped polystyrene microsphere among a static solution of nanoparticles at a laser power of $0.49 \mathrm{~W}$. The $\mu$ PIV laser is on and therefore the nanoparticles fluoresce. No trapping of the nanoparticles is observed due to the shield created by the size of the microsphere. 
Since the trapped sphere is sufficiently larger than the region of influence of the OT, the dynamic collection of nanoparticles at the trap location noted in the previous chapter is not observed. Brownian motion with a time average zero velocity is measured with $\mu \mathrm{PIV}$ for the surrounding nanoparticles. Therefore, for particle/cell studies, if the trapped particle/cell covers the OT region of influence, the effects of the OT on $\mu$ PIV measurements is negligible.

\subsubsection{TRAPPED SPHERE IN MICROCHANNEL FLOW}

Under dynamic conditions, a single sphere was trapped in either the middle of a straight microchannel with an imposed, gravity driven laminar flow or in a uniform flow. Figure $42 a$ shows the measured velocity field in the vicinity of a trapped $28 \mu \mathrm{m}$ sphere (every third vector is shown for clarity) in an imposed, gravity driven microchannel flow. This flow is more complicated than a uniform flow as a result of a non-uniform free stream velocity and significant wall effects. The measured velocity field is qualitatively and quantitatively consistent with the computationally predicted velocity field (Figure 42b). 

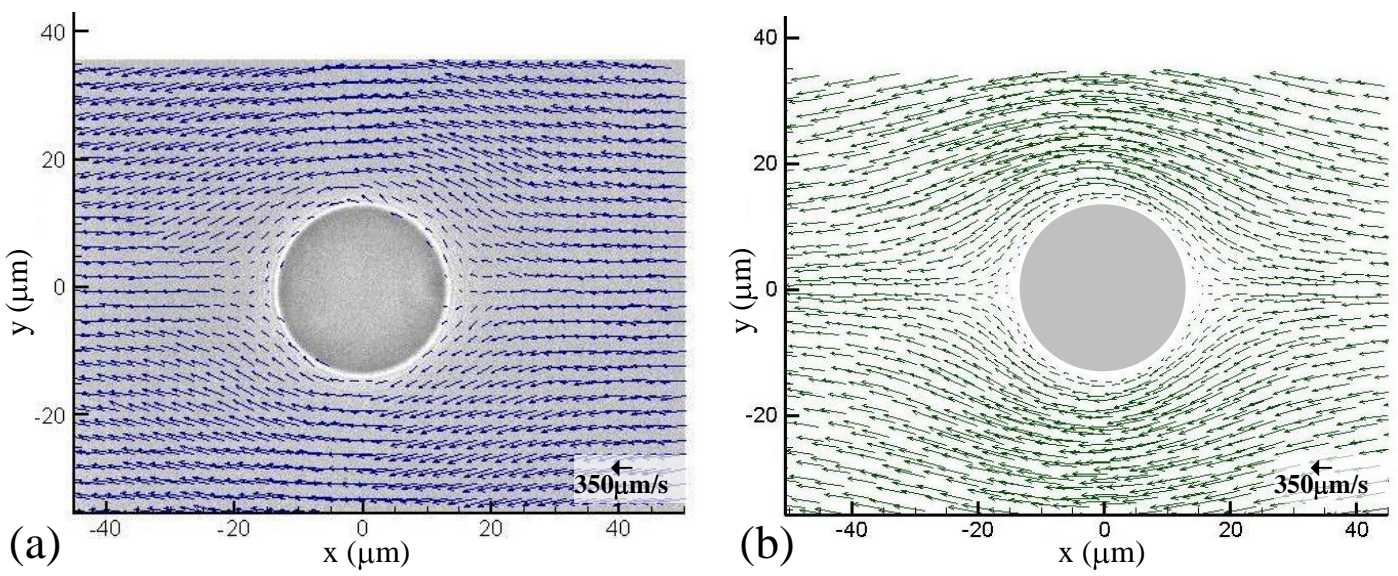

Figure 42: Comparison between the experimental velocity field and the computational prediction for a stationary sphere held in a gravity driven flow. (a) $\mu$ PIV 2-D velocity field around a trapped $28 \mu \mathrm{m}$ diameter polystyrene microsphere held in a straight flow. (b) Model simulation of the experimental flow conditions.

For quantitative comparison, Figure 43 shows the predicted and measured velocities along the inflow and outflow axes ( $x$ axis at $y=z=0$ in Figure 34).

As expected, the $\mathrm{x}$-direction velocity at the sphere surface (when $\mathrm{x} / \mathrm{a}$ is equal to 1 and -1 ) is zero (no-penetration condition) and the velocity increases towards its freestream velocity away from the sphere. Figure 43 shows strong quantitative agreement between the predicted and observed velocity as a function of dimensionless distance $(x / a)$ from the sphere $\left(R^{2}=0.988, R M S\right.$ Error $=13.04 \mu \mathrm{m} / \mathrm{s}$ along the inflow axis and $R^{2}=$ 0.973, RMS Error $=18.89 \mu \mathrm{m} / \mathrm{s}$ along the outflow axis). While not shown for brevity, the measured and predicted velocities along the cross-flow axis ( $y$ axis at $x=z=0$ in Figure $34 a$ and b) agree quantitatively as well. 


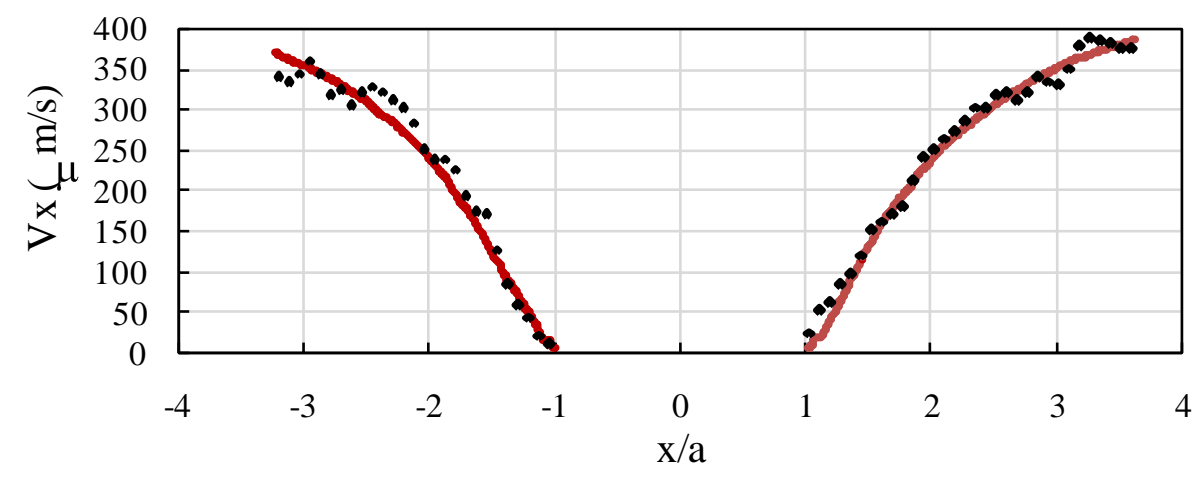

Figure 43: Comparison between measured (points) and predicted (line) velocities for a $28 \mu \mathrm{m}$ sphere in a gravity driven flow with a maximum plane velocity of $460 \mu \mathrm{m} / \mathrm{s}$.

\subsubsection{TRAPPED SPHERE IN UNIFORM FLOW}

Figure 44 quantitatively compares the measured and predicted velocities along the inflow and outflow axis of a stationary sphere in a uniform flow generated by translation of the automated microscope stage. With the velocity normalized by the free stream velocity (in this case the stage velocity) and the distance from the sphere normalized by the sphere radius, low Reynolds number hydrodynamics predicts the velocities collapse to a single curve for a sphere positioned the same dimensionless distance from the wall. Figure 44a shows the measured velocities collapse to a single curve for free stream velocities of 50 to $500 \mu \mathrm{m} / \mathrm{s}$, while Figure $44 \mathrm{~b}$ shows the velocities collapse for sphere diameters of $15 \mu \mathrm{m}$ to $35 \mu \mathrm{m}$. Figure 44 shows strong quantitative agreement between the predicted and observed velocity as a function of dimensionless distance $(x / a)$ from the sphere for diameters of $15 \mu \mathrm{m}$ to $35 \mu \mathrm{m}\left(R^{2}=0.9131, R M S=\right.$ 0.0776 along the inflow axis and $\mathrm{R}^{2}=0.9311, \mathrm{RMS}=0.0652$ along the outflow axis) and 
for free stream velocities of 50 to $500 \mu \mathrm{m} / \mathrm{s}\left(R^{2}=0.9310, \mathrm{RMS}=0.0635\right.$ along the inflow axis and $\mathrm{R}^{2}=0.9389, \mathrm{RMS}=0.0605$ along the outflow axis).

Note the discrepancy between the experimental velocities and computational predictions with the theoretical predictions of a sphere in a uniform flow without wall effects. The significant wall effects of the bottom coverslip force the actual velocity profile to reach the free stream velocity sooner. Thus, the $\mu$ PIVOT is capable of resolving the hydrodynamic interaction between the suspended sphere and the bottom wall.
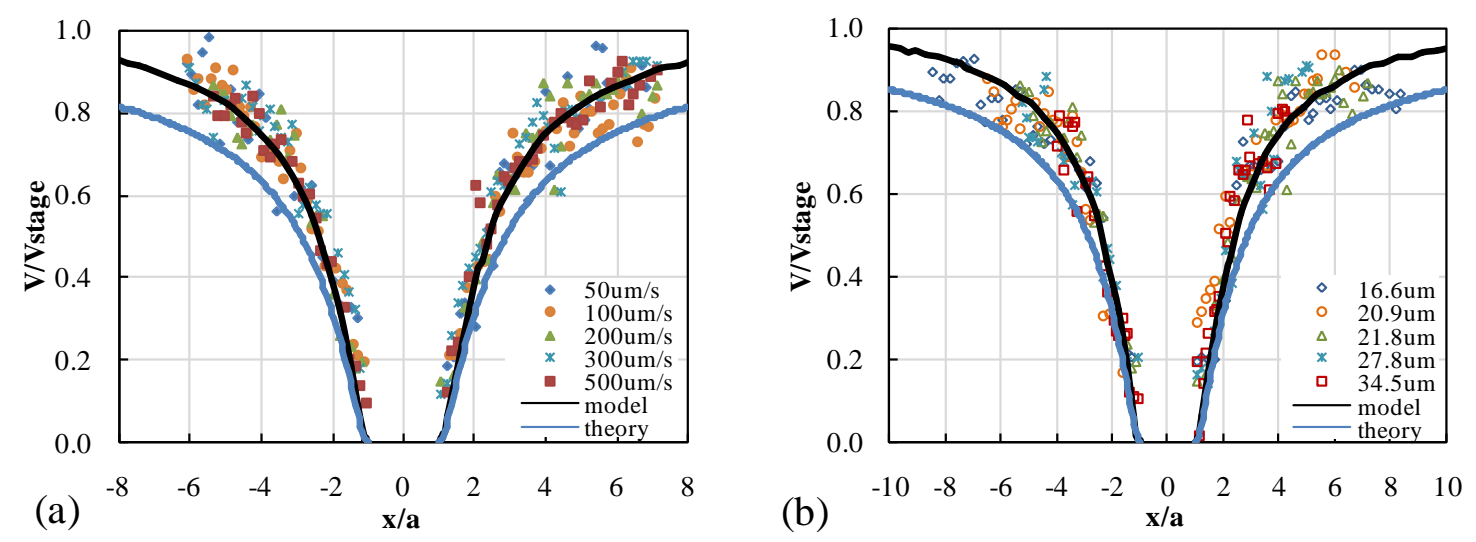

Figure 44: Quantitative comparison between measured velocities (points), computationally predicted velocities (line), and theoretical velocities without wall effects (dashed line). The inflow and outflow axis (- and $+x$ axis respectively) is normalized with the sphere radius, $a$, and the velocity is normalized with the automated stage velocity. (a) A $21.8 \mu \mathrm{m}$ sphere in a uniform flow at different velocities. (b) Different diameter microspheres with a constant uniform flow of $200 \mu \mathrm{m} / \mathrm{s}$. 


\subsubsection{TRAP STIFFNESS MEASUREMENTS}

Using the uniform flow set up with polystyrene microspheres, the trap stiffness was evaluated for different sphere diameters and at different depths. Fifty-one spheres of diameters ranging from 3.3 to $35.4 \mu \mathrm{m}$ were tested at depths of one radius away from the coverslip to approximately 10 radii away.

\subsubsection{Trap stiffness as a function of sphere diameter}

Figure 45 shows the measurements of the displacement of several sizes polystyrene spheres undergoing different flow velocities, hence different drag forces. Not all the spheres tested are represented on these graphs for clarity, but they cover the range of diameters studied. Figure 45a shows the linear portion of the drag force as a function of sphere displacement for each sphere. This linear portion corresponds to the data taken for drag forces up to approximately $40 \%$ of the maximum force before the sphere escapes. The slope of each curve represents the trap stiffness $(k)$ for that sphere. It is clear from this graph, that smaller spheres obtain higher trap stiffnesses. The trap is therefore more efficient for smaller spheres (this remark is valid only in the Mie regime). The correlation between trap stiffness and sphere diameter still has to be ascertained. The sphere diameter was the only variable in these experiments. The refraction indices of medium and object trapped were identical, along with laser intensity, power, wavelength, etc. 


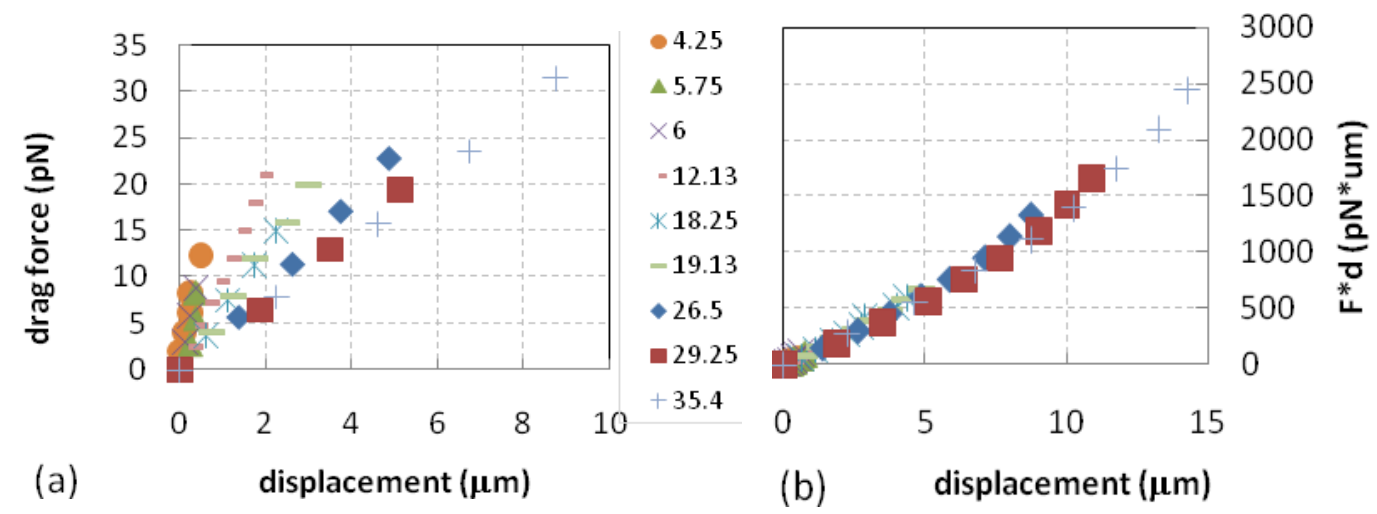

Figure 45: Trap stiffness dependence with the sphere diameter. (a) drag force versus sphere displacement curves for different diameter spheres. The slope of each curve represents the trap stiffness for the corresponding sphere. (b) When the drag force is multiplied by the sphere diameter, the curves all collapse into a single curve.

It was discovered that when the drag force was multiplied by the sphere diameter, the curves of F.d versus the sphere displacement all collapsed into one curve (Figure 45b).

Figure 46 shows the trap stiffness as a function of sphere diameter. The trap stiffness is seen to be directly proportional to the sphere diameter (d), with $k=C / d$, where $C$ is an empirical constant equal to $161.3\left(R^{2}=0.971\right)$. This correlation is to be expected in the Ray optics regime (Mie regime), where the lateral trap stiffness is given by [Mazolli, 2003]: $\quad k=-\frac{n_{1} P}{c} \frac{\partial Q_{\rho}}{\partial(\rho)}, \rho=0$

where $P$ is the laser power at the sample, $n_{1}$ is index of refraction of the medium, $c$ is the speed of light, $Q$ is the dimensionless trapping efficiency, $\rho$ is the distance from the beam axis. 


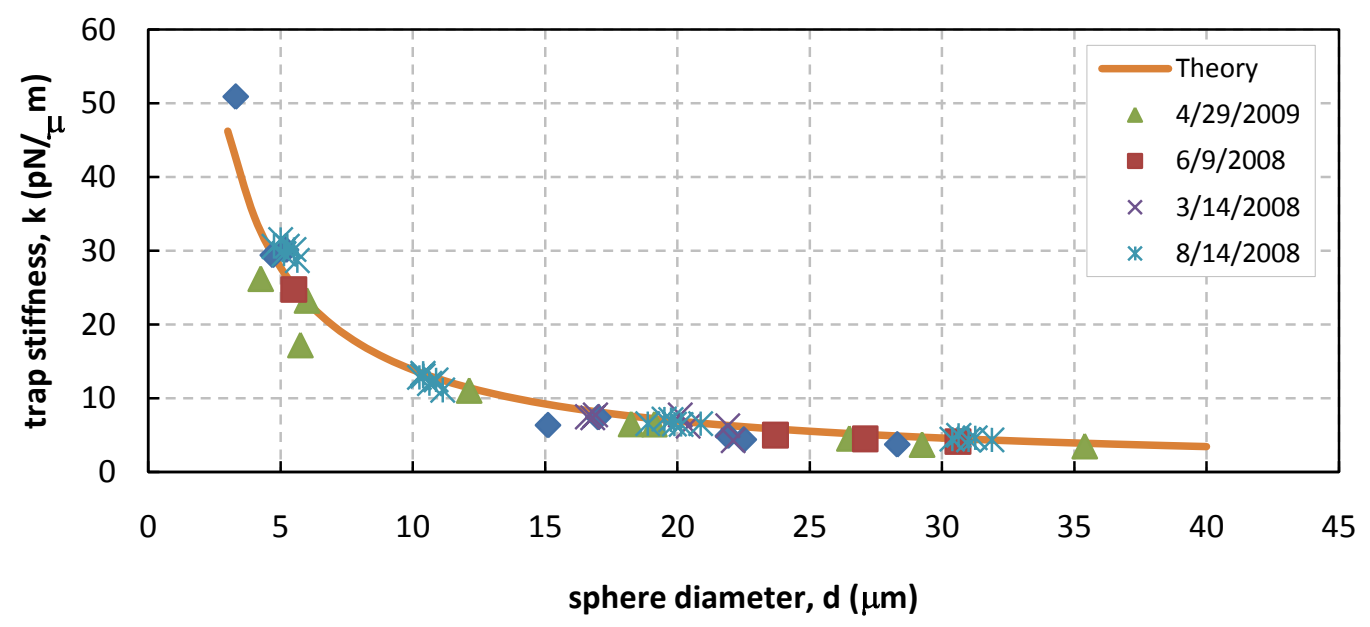

Figure 46: Trap stiffness versus sphere diameter measured in uniform flow experiments with an OT power of $35 \mathrm{~mW}$. Experiments made before and after the modifications made to the $\mu$ PIVOT setup are consistent and show good agreement with theory.

According to Mazolli et al., $Q_{\rho}$ can be decomposed into $Q_{s \rho}$ (momentum carried away by scattered field) and $Q_{e \rho}$ (momentum removed from the input beam) with:

$$
\begin{aligned}
Q_{s \rho}=4 \frac{\gamma^{2}}{A} \operatorname{Im} & \sum_{j, m}\left\{\frac{\sqrt{j(j+2)(j+m+1)(j+m+2)}}{j+1}\right. \\
& \times\left[\left(a_{j} a_{j+1}^{*}+b_{j} b_{j+1}^{*}\right)\left(G_{j, m} G_{j+1, m+1}^{*}+G_{j,-m} G_{j+1,-m-1}^{*}\right)\right] \\
& \left.-2 \frac{2 j+1}{j(j+1)} \sqrt{(j-m)(j+m+1)} \operatorname{Re}\left(a_{j} b_{j}^{*}\right) G_{j, m} G_{j, m+1}^{*}\right\}
\end{aligned}
$$

and

$$
Q_{e \rho}=2 \frac{\gamma^{2}}{A} \operatorname{Im} \sum_{j, m}\left\{(2 j+1)\left(a_{j}+b_{j}\right) G_{j, m}\left(G_{j, m+1}^{-}-G_{j, m-1}^{+}\right)^{*}\right\}
$$


where $A$ is the fraction of available beam power that fills the objective aperture, $\gamma=f / w$, focal length over beam waist, and $G_{j, m}$ is a multipole coefficient of the focused incident beam. Although these equations are complex, in the Ray optics approximation, $Q_{\rho}$ only depends on $\rho$ and is directly proportional to $\rho / a$. When $Q_{\rho}$ is derived the trap stiffness becomes:

$$
k=-\frac{n_{1} P}{c} \frac{\partial Q_{\rho}\left(\frac{\rho}{a}=0\right)}{\partial\left(\frac{\rho}{a}\right)} \frac{1}{a}
$$

The trap stiffness is therefore directly proportional to $\frac{1}{a}$, with

$$
k=\frac{D}{a}
$$

with $D=-\frac{n_{1} P}{c} \frac{\partial Q_{\rho}\left(\frac{\rho}{a}=0\right)}{\partial\left(\frac{\rho}{a}\right)}$, a constant depending on the system setup. For our system,

$$
D=\frac{C}{2}=80.65
$$

Mazolli et al. [Mazolli, 2003] used these equations to predict the trap stiffness as a function of diameter for defined parameters. Earlier, [Ghislain, 1994] measured the trap stiffness of an optical trap for experiments using polystyrene spheres in uniform flows. The spheres had different sizes, and the laser was a $1064 \mathrm{~nm} \mathrm{Nd:YAG} \mathrm{laser} \mathrm{(same}$ wavelength as in our experiments). The medium was also water and they measured the laser power to be $60 \mathrm{~mW}$. Although slightly higher, this power is in the same order of 
magnitude as the one we have been using in our experiments. Mazolli [Mazolli, 2003] used Ghislain [Ghislain, 1994]'s parameters, and calculated a theoretical trap stiffness equal to:

$\mathrm{k}_{\text {theory }}=541 / \beta \mathrm{pN} / \mu \mathrm{m} . \beta$ is the size parameter and is equal to the product of the wave number $\left(k_{\#}\right)$ and the sphere radius $(a)$ :

$$
\beta=k_{\#} a=\frac{2 \pi n_{1}}{\lambda} a=\frac{2 \pi 1.33}{1.064} a=7.8 a
$$

Therefore, $k_{\text {theory }}=541 / 7.8 \cdot a=69.4 / a$

$$
k_{\text {theory }}=139 / d
$$

When plotted against our results (Figure 46), the data shows very good agreements.

Finally, the data points in Figure 46 have been taken before and after the $\mu$ PIVOT system was modified for automation. This result shows a strong consistency and stability of our system.

Using the drag force and corresponding displacement data, the potential well shape can also be extracted for each sphere. With the force being conservative, we can define its potential $(U)$ as:

$$
U=\frac{1}{2} k \cdot \Delta x^{2}=\frac{1}{2} F_{d r a g} \Delta x
$$


Figure 47 shows the potential wells for different sphere diameters. The displacement $\Delta x$ was measured in only one direction (positive $\mathrm{x}$ direction). The value 0 corresponds to the static position of the sphere. To obtain negative $x$ positions, the data were mirrored. Test experiments verify the trap stiffness is isotropic.

The potential well is wider for larger spheres and gets narrower for smaller spheres. Again, this conclusion is valid only in the ray-optics approximation. The results shown in Figure 47 corroborate with the results of Malagnino [Malagnino, 2002].

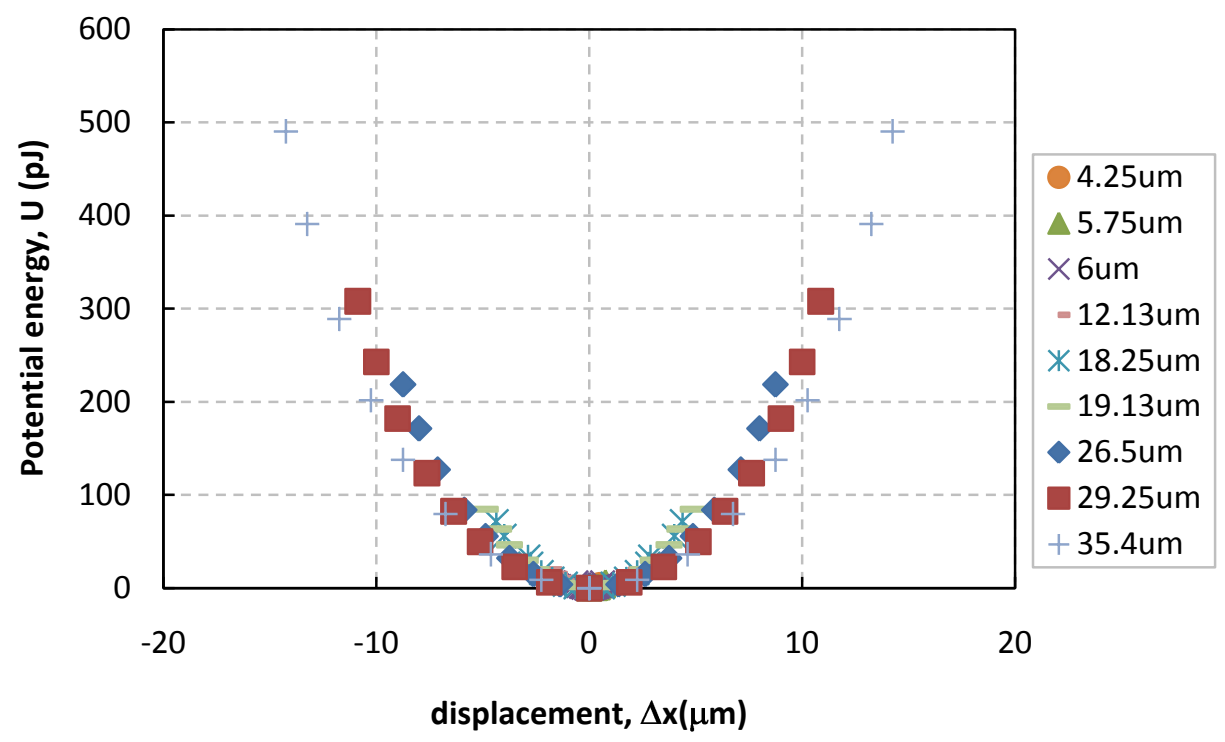

Figure 47: Potential wells for different sphere diameters 


\subsubsection{Trap stiffness as a function of distance from the coverslip}

The trap stiffness of an $11 \mu \mathrm{m}$ diameter sphere was tested at different distances from the coverslip. The distance to the bottom coverslip was calculated by first identifying the $z$ position of the coverslip. This was achieved by focusing the objective lens on a sphere resting at the coverslip. The wall height is simply at a radius away from that focus plane. When the objective lens is moved up into the sample, its position is recorded. The bead height is then calculated with: $h_{\text {bead }}=\left(n_{\text {water }} / n_{\text {glass }}\right) \cdot h_{\text {focus }}$. With $n_{\text {water }}$ $\left(=n_{1}\right)$ and $n_{\text {glass }}$ being the refractive indices of water and glass respectively [Vermeulen, 2006]. As in previous measurements, the drag force was calculated from knowing the sphere diameter, its distance from the wall, and the flow velocity. Figure 48 shows the drag force as a function of sphere displacement at varying depths.
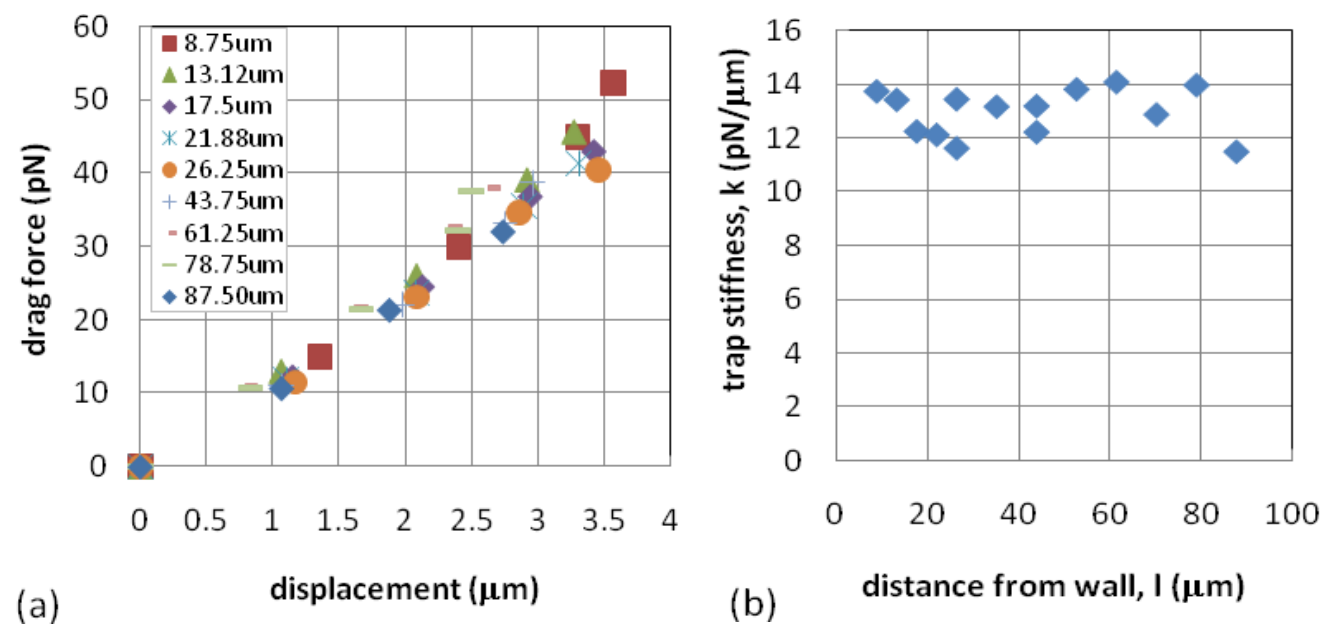

Figure 48: (a) Drag force versus displacement for an $11 \mu \mathrm{m}$ sphere at different distances from the bottom coverslip. (b) Resulting trap stiffness as a function of distance from the wall. 
As shown in Figure 48a, the curves overlap. The trap stiffness was averaged to be 13.0 $\pm 0.9 \mathrm{pN} / \mu \mathrm{m}$. This result of trap stiffness is consistent with Figure 46 . However, the trap stiffness being independent of depth is surprising. Previous studies have shown that the trap stiffness decreases with distance from the bottom coverslip [Vermeulen, 2006], and the trapping force is theoretically insufficient to hold a microsphere at several radii away [Fallman, 2003], [Rohrbach, 2002]. They attribute this limitation to optical aberrations due to the index of refraction mismatch at the glass-water interface (i.e. the coverslip/flow medium interface). This media interface causes the rays with large incident angles to focus in front of those with small angles. This elongates the focal spot. A particle trapped would then not "capture" all of the rays and would be trapped more weakly. Regarding Figure 48 however, we have shown that for our system, beads trapped at 9 radii away from the coverslip were still efficiently trapped.

\subsubsection{EXTENSIONAL FLOW}

Figure 49 shows a $20.6 \mu \mathrm{m}$ diameter polystyrene sphere suspended in a cross junction flow. The velocity field is measured with the $\mu$ PIV function of the $\mu$ PIVOT to characterize the local flow state. In this type of flow, the linear velocities vary with distance from the stagnation point with the simple relationship:

$$
\left\{\begin{array}{c}
v_{x}=\dot{\gamma} x \\
v_{y}=-\dot{\gamma} y
\end{array}\right.
$$




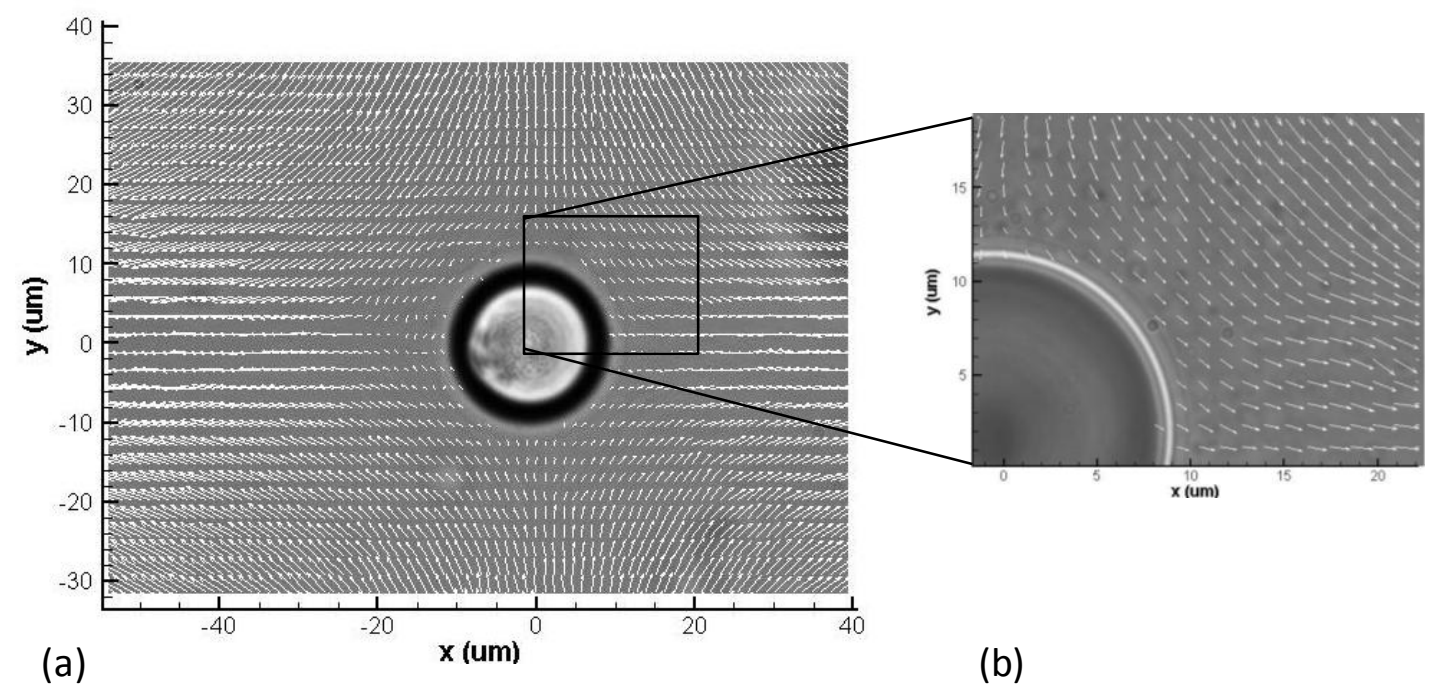

Figure 49: (a) Velocity flow field surrounding an analogous cell (20.6 $\mu \mathrm{m}$ diameter polystyrene microsphere) as measured with micron-resolution particle image velocimetry. (b) zoom in.

Where $v_{x}$ and $v_{y}$ are the velocities along the $x$ (outflow) and $y$ (inflow) directions respectively, and $\dot{\gamma}$ is the extension rate. With a pressure head of $24.5 \mathrm{~mm}$ and $P_{\infty}=-239.5 \mathrm{~Pa}$ (pressure), a gravity driven flow was generated with a far-field channel, centerline mid-plane velocity of $750 \mu \mathrm{m} / \mathrm{s}$ and a local hydrostatic pressure of $\mathrm{p}=1 / 2 P_{\infty}=$ $-119.75 \mathrm{~Pa}$. This produced a mid-plane extension rate of $\dot{\gamma}=12.4 \mathrm{~s}^{-1}$ at the crossjunction (determined without microsphere perturbation).

Theoretically, if a sphere is positioned perfectly at the stagnation point, no trapping force is required to maintain its position, regardless of the applied shear rate, effectively creating a hydrodynamic trap. In reality, the stagnation point represents a saddle point and is unstable to perturbations. The trapping force acts as a restoring 
force and does not need high powers to keep the microsphere in place. This experiment is the first reported full-field velocity measurement around a sphere placed at the stagnation point of a cross-junction channel.

\subsection{DISCUSSION}

Interference between the OT and $\mu$ PIV techniques has been visualized when trapping polystyrene spheres. The nanoparticle agglomeration process observed in Chapter 4 is usually negligible when a polystyrene sphere is trapped (and thus shields the nanoparticles from the trap). However, two circumstances exist where the influence is significant and OT interferes with $\mu$ PIV. Both of these occur when the nanoparticles are not excluded from the optical trap region of influence. First, when the trapped particle is smaller than the region of influence, nanoparticles are attracted towards the center of the trap. Their concentration at that spot becomes problematic due to the fact that they are excited by the OT laser which induces image saturation. Second, when the trapping power is relatively low and a large sphere is trapped in a sufficiently fast flow, the region of influence may extend beyond the sphere. During flow, the imposed drag force displaces the particle relative to the trap center (trap stiffness behavior). If the flow is sufficiently fast, the particle displacement may approach the sphere radius which means the trap center is located near the sphere edge. If the trap region of influence extends beyond the sphere edge, nanoparticles may be attracted and conglomerate. This results in a saturated image on the inflow axis. Regardless, these two cases of 
significant interference can be mitigated with the proper selection of trap power and particle size. Overall, the quantitative agreement between the measured and predicted velocities, shown in Figure 44 and Figure 43, indicates limited influence of OT on $\mu$ PIV during flow conditions and validates the integration of OT and $\mu$ PIV for trapped particle diameters of 15 to $35 \mu \mathrm{m}$ and velocities from 50 to $500 \mu \mathrm{m} / \mathrm{s}$.

\subsection{SUMMARY}

The integrated techniques were validated by comparing computational predictions to the measured velocity profile around a trapped particle in either an imposed microchannel flow or a uniform flow. Good quantitative agreement between measured and predicted velocities is observed for 15 to $35 \mu \mathrm{m}$ diameter trapped particles subjected to fluid velocities from $50 \mu \mathrm{m} / \mathrm{s}$ to $500 \mu \mathrm{m} / \mathrm{s}$ even at the highest laser power $(1.45 \mathrm{~W})$. This validation demonstrates the first reported full field velocity measurements around a freely suspended particle in either an imposed microchannel flow, a uniform flow or an extensional flow.

Individually, the Optical Trap system was characterized using polystyrene spheres. The trap stiffness was shown to be inversely proportional to the sphere diameterm $\mathrm{k}=\mathrm{C} / \mathrm{d}$, and independent of depth to several radii from the coverslip. 


\section{CHAPTER 6}

\section{UNIFORM FLOW AROUND TWO SUSPENDED SPHERES}

Particle-particle and fluid-particle hydrodynamic interactions play a key role in many natural and industrial processes. The subtle interactions between particles and suspending fluids yield fluids with unique rheology as well limit potential applications of suspensions in material processing. Understanding the behavior of hydrodynamic interactions in Newtonian and non-Newtownian fluids is critical for theoretical and computational model development especially with the push towards "nano"-particle enhanced materials and fluids.

While a number of a studies have focused on the interaction among spheres, the effects of a second sphere on the drag and lift forces exerted on a reference sphere is still poorly understood. Theoretical results only exist for spheres placed in tandem or side-by-side with respect to the main flow direction. Computational results are also primarily focused on similar sphere arrangements (for validation of the computational model with theoretical predictions). A noticeable exception, Yoon et al. [Yoon, 2007] modeled 2-sphere interactions with multiple configurations at a Reynolds number $R e$ equal to 300. Their work focused on the development of vortex shedding. 
The most common methods to identify hydrodynamics interactions

experimentally are either 1 ) placing two spheres at a set orientation in a quiescent fluid, releasing the spheres, and mapping their trajectories or 2) mounting particles/spheres to thin rods with force transducers and measuring the force for different flow fields and particle orientations. The first experimental studies regarding spheres interacting at low Reynolds number were performed by Eveson et al. [Eveson, 1959] who studied the relative velocity of two spheres falling at different angles in a highly viscous fluid. Note, for our experiments, with the fluid properties of water, a sphere diameter of $12 \mu \mathrm{m}$, and uniform velocity of $200 \mu \mathrm{m} / \mathrm{s}$, the Reynolds number is quite small, approximately 0.002 . Therefore, low Re studies are most appropriate. Happel and Pfeffer [Happel, 1960] reported the increase in the terminal velocity for two particles falling along one axis compared to an isolated particle under the same conditions. More recently, Chen and Lu [Chen, 1999] studied the drag force of an interactive particle. They measured the drag coefficients of spheres placed at different angles and distances from each other (groups of 2 and 3 spheres were studied) by placing and holding spheres in a uniform flow by thin rods. They showed the drag force depended strongly on the instantaneous flow field around the particle. Following a similar technique, Chen and Wu [Chen, 2000] experimentally investigated the influences of a nearby sphere on the drag flow characteristics of a test sphere. 
With the $\mu$ PIVOT, theoretical models characterizing microscale fluid and particle phenomena may be verified experimentally, and previously unattainable measurements of direct particle-particle interactions of optically suspended spheres may be obtained. With its dual trap capability, the $\mu$ PIVOT enables the suspension of two particles in an imposed fluid flow and the simultaneous measurement of the velocity field surrounding the interacting particles and resulting drag force. From these measurements the hydrodynamic interaction and the detailed flow characteristics required for validating suspension and non-Newtonian constitutive equations may be determined.

This chapter examines the interaction of freely suspended spherical particles in uniform flows. The velocity field in the vicinity of the two interacting spheres is measured with $\mu$ PIV and the drag reduction which develops as a result of the hydrodynamic interaction is quantified. These initial two spheres studies agree with low Re hydrodynamic models in Newtonian fluids and provide the basis for future studies with non-Newtonian fluids.

\subsection{EXPERIMENTAL PROTOCOL}

\subsubsection{EXPERIMENTAL SETUP}

A uniform flow was generated by placing a reservoir containing a very diluted concentration of polystyrene spheres on the automated translation stage and moving the stage at a constant unidirectional velocity $(200 \mu \mathrm{m} / \mathrm{s})$. Using the $\mu$ PIVOT's ability to 
traps two objects at any locations, two spheres of approximately the same size

(approximately $12 \mu \mathrm{m}$ diameter) were positioned with different separation distances

and angles from the flow. The leading sphere position was fixed during all experiments.

Spheres were initially positioned by calculating the leading sphere position $\left(x_{l}, y_{l}\right)$ and

trailing sphere position $\left(x_{t}, y_{t}\right)$ for desired separation distances, $d$, and flow angle, $\theta$

(angle between the flow direction and spheres centerline).

$$
\left\{\begin{array}{c}
x_{t}=\frac{d}{\sqrt{1+\tan ^{2}\left(\frac{\pi}{180} \theta\right)}+x_{l}} \\
y_{t}=-d \sqrt{1-\frac{1}{\tan ^{2}\left(\frac{\pi}{180} \theta\right)+1}}+y_{l}
\end{array}\right.
$$

Figure 50 shows the five different flow angles $(0,30,45,60$, and 90 degrees) examined with the separation distance varying between 1 and 5 sphere diameters.

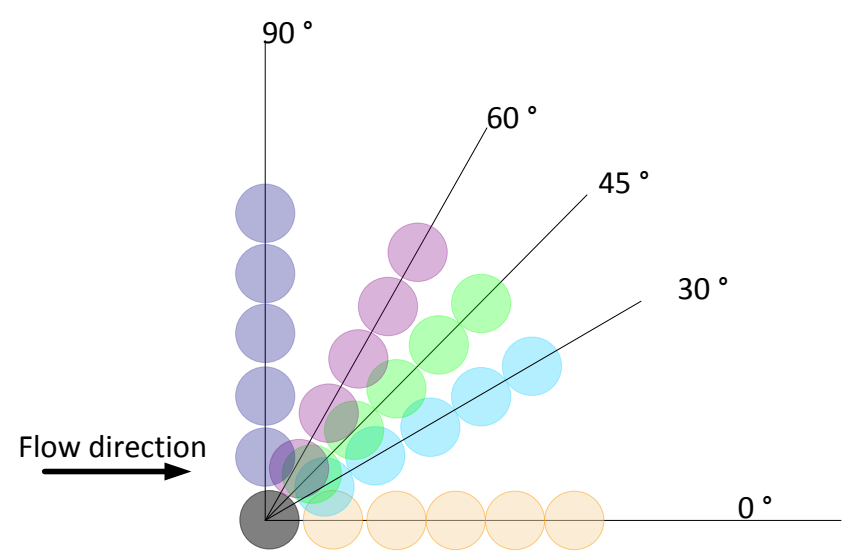

Figure 50: Sketch of the different configurations. The leading sphere, at the center of the axis was held stationary while the trailing sphere was positioned at various distances from the leading sphere (up to 5 diameters away), with their centerline forming various angles from the flow axis. 
Images of the two spheres were taken with and without an imposed flow. With an imposed uniform flow at $200 \mu \mathrm{m} / \mathrm{s}$ the sphere locations $\left(x_{l}, y_{l}\right)$ and $\left(x_{t}, y_{t}\right)$ were measured and the separation distance $(d)$ and the flow angle $(\theta)$ were calculated.

$$
\begin{gathered}
d=\sqrt{\left(x_{t}-x_{l}\right)^{2}+\left(y_{t}-y_{l}\right)^{2}} \\
\theta=\frac{180}{\pi} \tan ^{-1}\left(\frac{y_{t}-y_{l}}{x_{t}-x_{l}}\right)
\end{gathered}
$$

Additionally, following the single sphere experimental procedures (Chapter 5), the displacement of each sphere from the trap location due to the flow was measured and the drag force on each particle calculated by knowing $\mathrm{k}$ and the displacement of each microsphere from the trap center, $F_{d r a g}=k \Delta x$ (Figure 51).

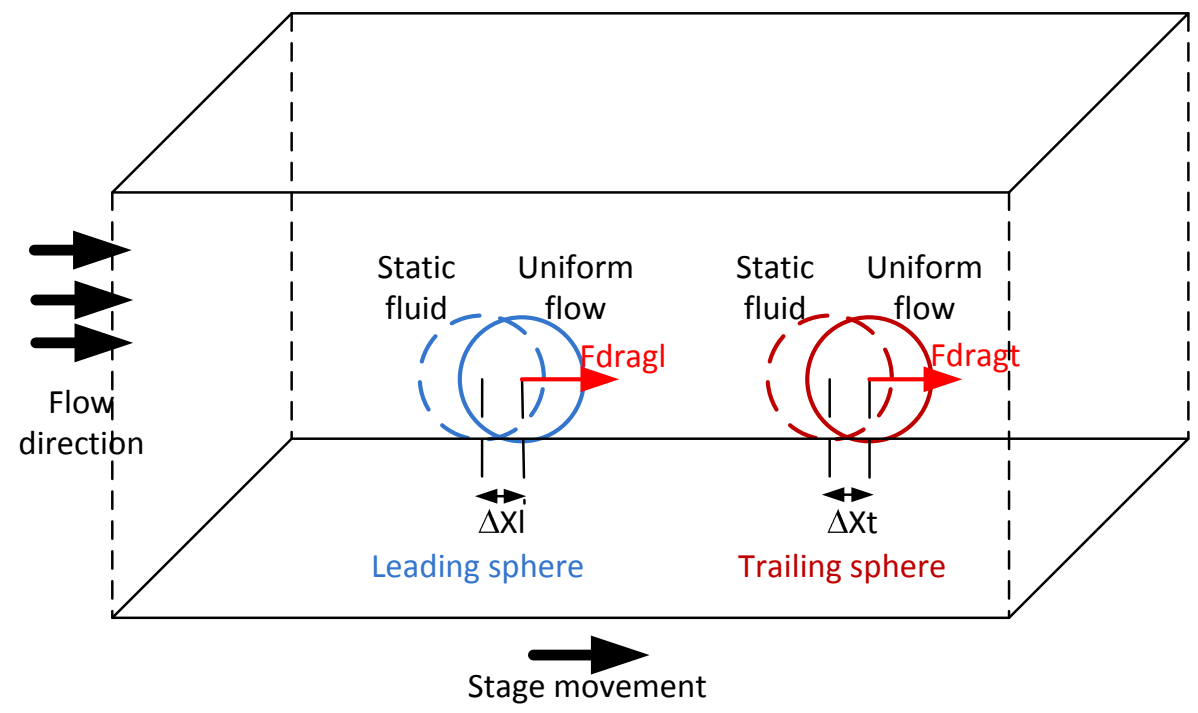

Figure 51: Schematic of the two spheres experiment. When the automated stage moves at constant velocity, the flow induces a displacement of each of the trapped spheres. 
Note, for each experiment, the trap stiffness $(\mathrm{k})$ and drag force $\left(F_{0}\right)$ were first measured for each sphere without the presence of a second sphere. This calibration determined the exact values of $\mathrm{k}$ and $F_{0}$ for each trap and sphere combination.

The drag force for a single sphere in a uniform flow $\left(F_{0}\right)$ is calculated to determine the reduced drag associated with the two sphere interaction. With the drag force of each sphere ( $F_{l}$ for the leading sphere and $F_{t}$ for the trailing sphere), the corresponding reduced drag for each sphere is $F_{l} / F_{0}$ and $F_{t} / F_{0}$. Traditionally, this dimensionless reduced drag is determined as a function of normalized distance between the spheres, $d / 2 a$, with $d$, the distance between the center of the spheres and $a$, the radius of the spheres. Note, at low Re Newtonian flows, the theoretical reduced drag force is equal for both the leading and trailing sphere regardless of the flow angle or distance between the spheres.

The measured normalized drag force for each sphere was compared to computational models for all separation distance and flow angles and to the theoretical model of Stimson and Jeffery [Stimson, 1926] that provides an exact solution using bipolar coordinates for two equal spheres with their centerline parallel to a $R e<3$ linear flow.

For the specific case of two spheres aligned with the flow direction, fluid velocities in the vicinity of the spheres were measured with $\mu$ PIV. Two spheres (leading 
sphere: $26.6 \mu \mathrm{m}$ in diameter, trailing sphere: $28.5 \mu \mathrm{m}$ in diameter) were trapped and positioned with their centerline parallel to the flow at a distance $d=42.18 \mu \mathrm{m}$ from each other. This provides a normalized distance of $d / 2 a=1.53$. The flow was seeded with fluorescent nanoparticles (concentration of $50 \mu \mathrm{L} 2 \%$ solids nanoparticle solution to $2 \mathrm{~mL}$ of distilled water) for $\mu$ PIV measurements around the microspheres. Image pairs were captured while the automated stage was moving at a velocity of $350 \mu \mathrm{m} / \mathrm{s}$. The $\mu$ PIV images were examined with an initial interrogation window of $64 \times 64$ pixels and $75 \%$ image overlap, then further refined to $32 \times 32$ pixels with a $50 \%$ overlap, leading to a velocity vector spacing of $1.74 \mu \mathrm{m}$. The time difference between images $(\Delta \mathrm{t})$ was set at $3 \mathrm{~ms}$. This time separation provided sufficient particle displacements and good image quality for the velocity vector analysis.

\subsubsection{COMPUTATOINAL MODELING}

For comparison with the experimental results, the two sphere interaction was modeled using the multiphysics software COMSOL Multiphysics ${ }^{\circledR}$ version 3.5a (COMSOL Inc., Burlington, MA). For the experimental conditions (microscale, small velocities), the steady flow is laminar $\left(R e \approx 10^{-3}\right)$. The fluid was assumed to be an incompressible Newtonian fluid with the properties of water at room temperature (density equal to $1000 \mathrm{~kg} / \mathrm{m}^{3}$ and viscosity equal to $10^{-3} \mathrm{Ns} / \mathrm{m}^{2}$ ). The flow domain was a rectangular parallelepiped of dimensions $x=260 \mu \mathrm{m}, y=260 \mu \mathrm{m}$, and $z=250 \mu \mathrm{m}$. 
The spheres were positioned to match the experimental separations and flow angles. The leading sphere was fixed at $(x=0, y=0, z=50 \mu \mathrm{m})$ while the trailing sphere was moved at increasing multiples of diameters away from the leading sphere (1 to 5) for flow angles of 0,45 , and 90 degrees. The bottom coverslip and side boundaries were modeled as no-slip wall boundaries moving at a constant velocity of $200 \mu \mathrm{m} / \mathrm{s}$. The flow inlet was set at a uniform $200 \mu \mathrm{m} / \mathrm{s}$ and the flow outlet prescribed as an open boundary with no viscous stress and a pressure equal to 0 . For each model, the computational domain was meshed with more than 55000 polyhedrals to provide a numerical resolution greater than experimental measurements. A refined mesh was incorporated near the spheres surface (Figure 52). The Navier-Stokes equation and mass continuity equation were then solved iteratively for the incompressible steady flow. From the resulting velocity field, the drag force on each sphere could be extracted.

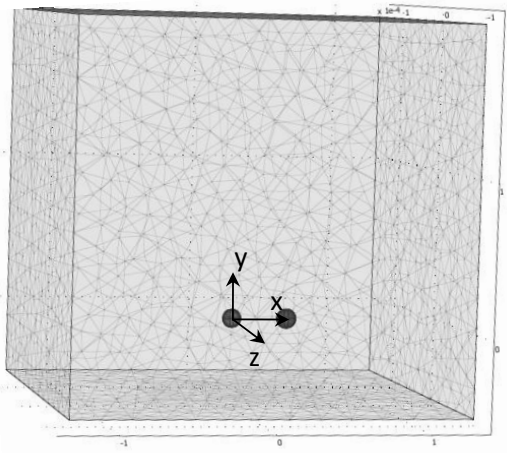

(a)

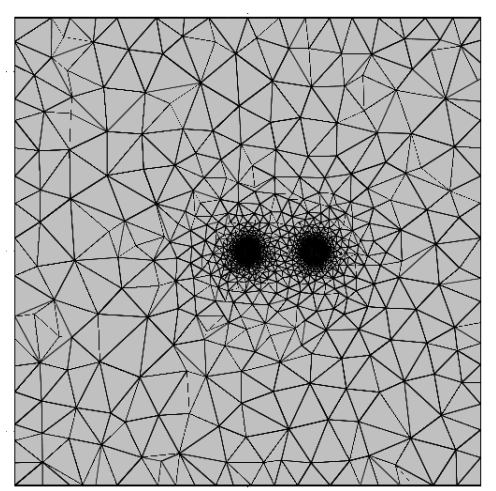

(b)

Figure 52: (a) Computational model of flow around two spheres. In this configuration, $d=3$ and $\theta=0$. The flow is going from left to right. (b) $x-y$ plane at $z=50 \mu \mathrm{m}$, going through the center of the spheres. The flow also comes from the left. The meshing was refined near the spheres. 


\subsection{RESULTS}

The drag forces of the leading sphere and the trailing sphere were studied for different distances and angles with a uniform flow velocity of $200 \mu \mathrm{m} / \mathrm{s}$. The spheres were $12 \mu \mathrm{m}$ in diameter for the leading sphere and $12.75 \mu \mathrm{m}$ in diameter for the trailing sphere. The average diameter (2a) was therefore $12.375 \mu \mathrm{m}$.

\subsubsection{DRAG FORCE OF TWO SPHERES IN A UNIFORM FLOW}

Flow around two equal size spheres with their centerline parallel to the flow axis was solved theoretically in 1926 by Stimson and Jeffery [Stimson, 1926]. The results are shown in Table 2.

\begin{tabular}{|c|c|}
\hline$d / 2 a$ & $F / F_{0}$ \\
\hline 1.128 & 0.663 \\
\hline 1.543 & 0.702 \\
\hline 2.352 & 0.768 \\
\hline 3.762 & 0.836 \\
\hline 6.132 & 0.892 \\
\hline 10.068 & 0.931 \\
\hline 100 & 1 \\
\hline
\end{tabular}

Table 2: Theoretical results from Stimson and Jeffery [Stimson, 1926].

With a more elaborate model Keh and Chen [Keh, 1997] computed the two spheres interaction with possible slip at the spheres surface included. Their no-slip results were consistent with Stimson et al. [Stimson, 1926]. 
Figure 53 shows the computed flow velocities in the $x-y$ center plane. The flow direction is from left to right. As expected, the flow is laminar and the flow velocities between the two spheres for a given distance higher at $\theta=90^{\circ}$ than for $\theta=0^{\circ}$. The distinct symmetry in the flow field near each sphere is a direct consequence of the linearity of the creeping flow equations. Thus, regardless of the two sphere orientation, the drag force on both the leading sphere and trailing sphere are identical. The drag force was extracted and compared to the experimental results and theoretical predictions. 
$\Theta=0^{0}$

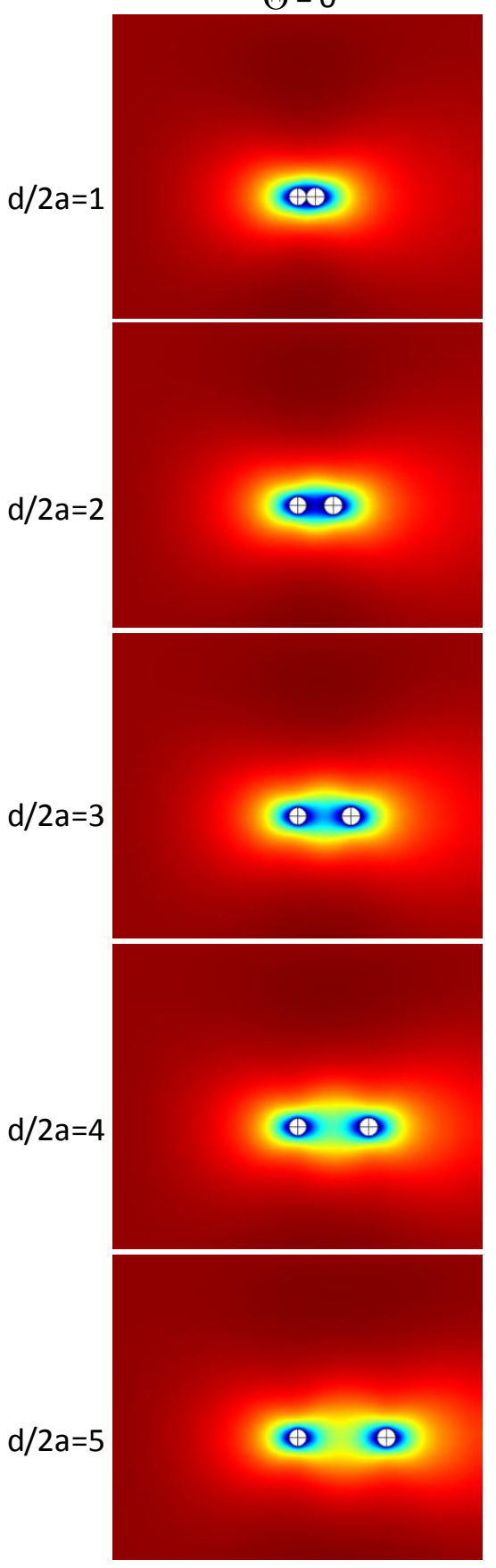

$\Theta=45^{\circ}$
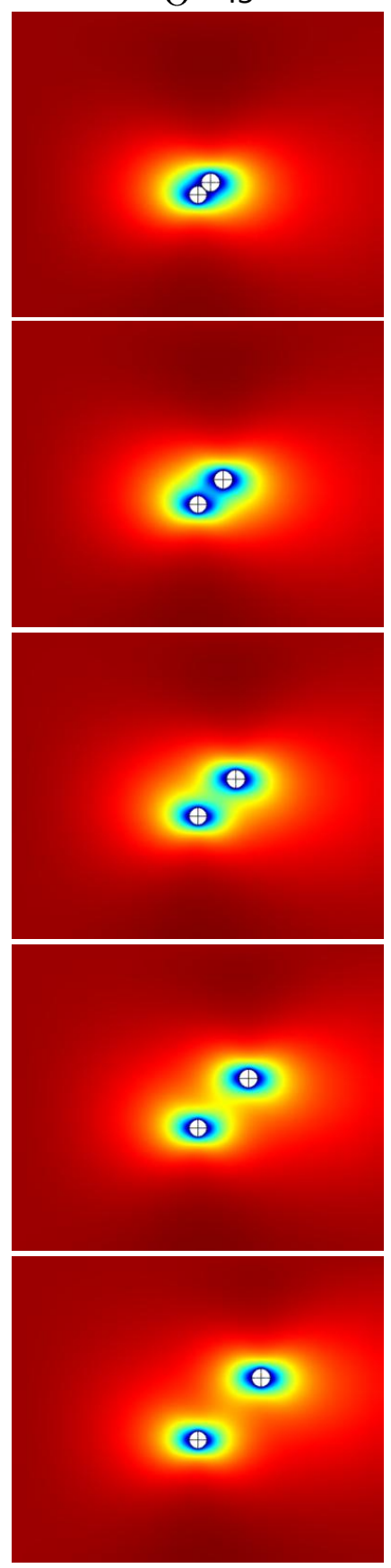

$\Theta=90^{\circ}$
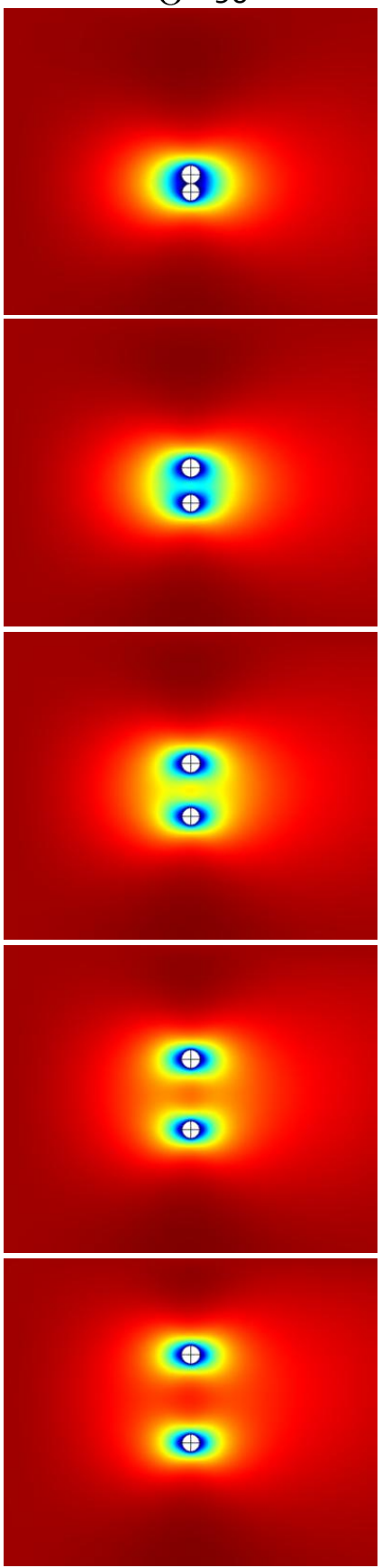

Figure 53: Computational model results for normalized distances from 1 to 5 and angles at 0,45 and 90 degrees. The images are taken in the $x-y$ plane at $z=50 \mu \mathrm{m}$ (going through the center of the spheres). Flow velocities range from 0 (dark blue, at the spheres surface) to the free stream velocity of $200 \mu \mathrm{m} / \mathrm{s}$ (dark red far away from the spheres). 
Figure 54 shows the theoretical, experimental, and computational reduced drag as a function of separation distance between the two spheres oriented with their centerline parallel to the flow $\left(\theta=0^{\circ}\right)$. This graph shows that the theoretical and computational curves and the experimental data are qualitatively similar with the reduced drag a minimum when the two spheres are in contact and increasing smoothly towards 1 (single sphere drag) as the separation distance increases.

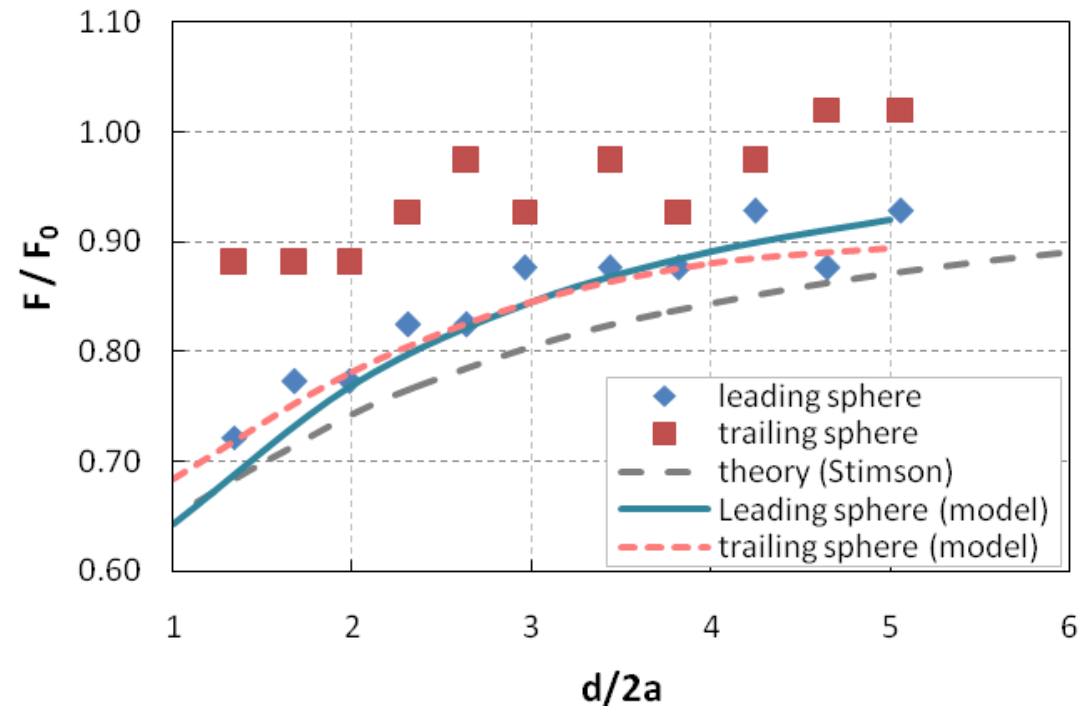

Figure 54: Normalized drag force as a function of normalized distance between the microspheres when their centerline is parallel to the flow axis $\left(\theta=0^{\circ}\right)$. 
However, quantitatively, significant differences exist. First, the computationally predicted reduced drags for the leading and trailing spheres are distinctly different. Theoretically, if the two spheres have identical diameters and the Re is identically zero, the reduced drags should be identical. The computations modeled the experimental conditions in which the Re was small but finite and spheres were similar but not identical in size. While the computationally predicted reduced drags are quite similar, it is unclear whether the slight difference in the computed reduce drag behavior is a result of the size discrepancy or is a function of the resolution of the computational mesh.

Second, when compared to the theoretical predictions of Stimson et al.[Stimson, 1926], both the leading and trailing sphere computational predictions show less drag reduction and a more rapid approach to single sphere drag. As stated above, the theoretical prediction assumes identical sized spheres in an infinite uniform flow under creeping flow conditions $(\operatorname{Re}=0)$. The computations, however, account for the size discrepancy and include a small but finite Re. More importantly, the computations impose the far field velocity at the bottom coverslip which is approximately 10 radii from the sphere. This distance is sufficiently close that the spheres hydrodynamically interact with the bottom cover slip and effectively increase the drag. For a dimensionless distance from the coverslip of 10 radii, the additional drag can be estimated (see Happel and Brenner drag force adjustment [Happel, 1983] in Chapter 2) to be approximately $10 \%$. This $10 \%$ consistent with the more rapid approach observed 
in the computational model. Thus, it is reasonable to assume that discrepancy between the theoretical prediction and the computations results from the finite distance to the bottom coverslip.

Finally and more intriguing is the apparent quantitative discrepancy between the computational predictions and the experimental results. Quantitatively, the experimental result and computations show good agreement for the leading sphere. However, for the trailing sphere the reduced drag is significantly higher (approximately $10 \%)$ than the leading sphere measurements and the computational predictions for both a leading and trailing sphere. The reason for this discrepancy is unclear. It may arise from errors in the experimental measurements. Specifically, the trailing sphere reduced drag exceeds 1 at a dimensionless distance above 4 . The reduced drag should approach 1 but never exceed it. Additionally, a significant scatter in the data (larger than the leading sphere) is observed. This may indicate an error associated with the trap stiffness during the measurements. If the trap stiffness of the trailing sphere trap is lowered by $10 \%$, the results are more quantitatively consistent with the leading sphere measurements and the computational predictions (see Figure 55). Interestingly, the apparent discrepancy in the trailing sphere measurements is present in all experiments examine regardless of the two sphere orientation or separation distance. 


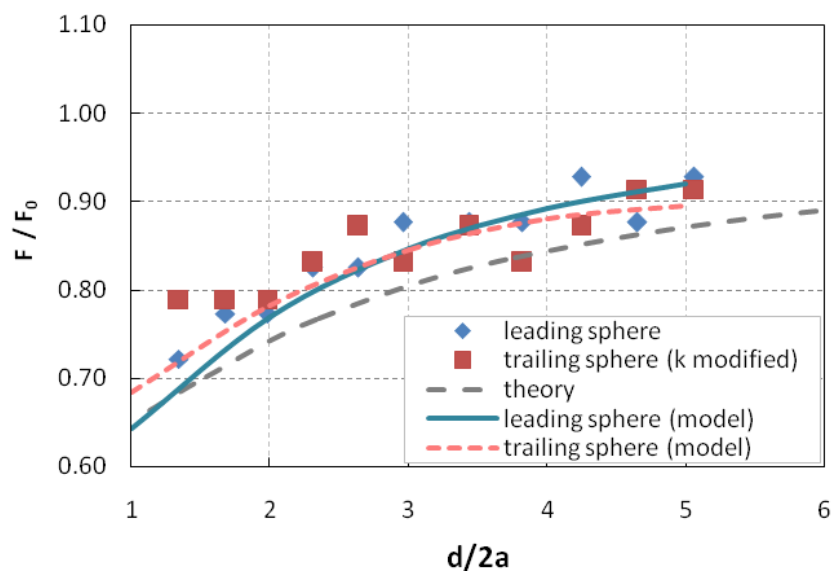

Figure 55: Normalized drag force as a function of normalized distance between the microspheres for $\theta=0^{\circ}$. The stiffness of the trap trapping the trailing sphere has been lowered by $10 \%$.

Figure 56 shows the reduced drag for sphere orientations of 45 and 90 degrees.

The trailing sphere drag force measurements were adjusted by a modified trap stiffness of $90 \%$. With this modification, Figure 56 shows that the predicted and measured reduced drags are not only qualitatively consistent but quantitatively accurate as well. Note, the scatter in the trailing sphere measurements in Figure 56 are reduced relatively to the scatter in Figure 55.
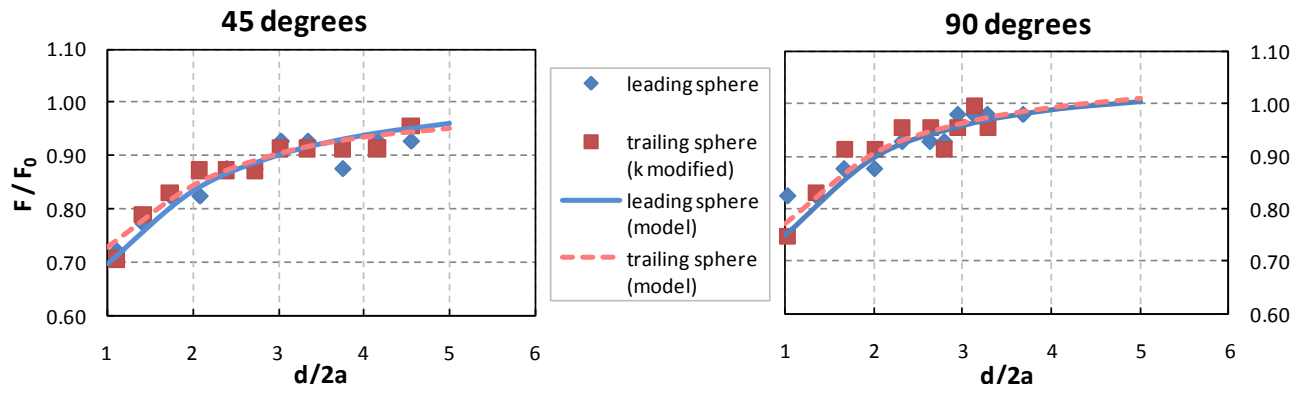

Figure 56: Normalized drag force as a function of normalized distance between the microspheres for angles of $\theta=45^{\circ}$ (left) and $\theta=90^{\circ}$ (right). The stiffness of the trap trapping the trailing sphere has been lowered by $10 \%$.

116 
Figure 54, Figure 55, and Figure 56 seem to indicate a trap discrepancy between the calibration and experimental results.

Figure 57 shows the leading sphere reduced drag as a function of distance between the spheres for different flow angles. The experimental results and computational predictions show the reduced drag increases with flow angle and approaches single sphere drag faster for higher angles. For $\theta=90^{\circ}$, the reduced drag reaches its maximum value at $d / 2 a=4$, whereas this plateau is still not reached at $d / 2 a$ $=6$ for $\theta=0^{\circ}$.

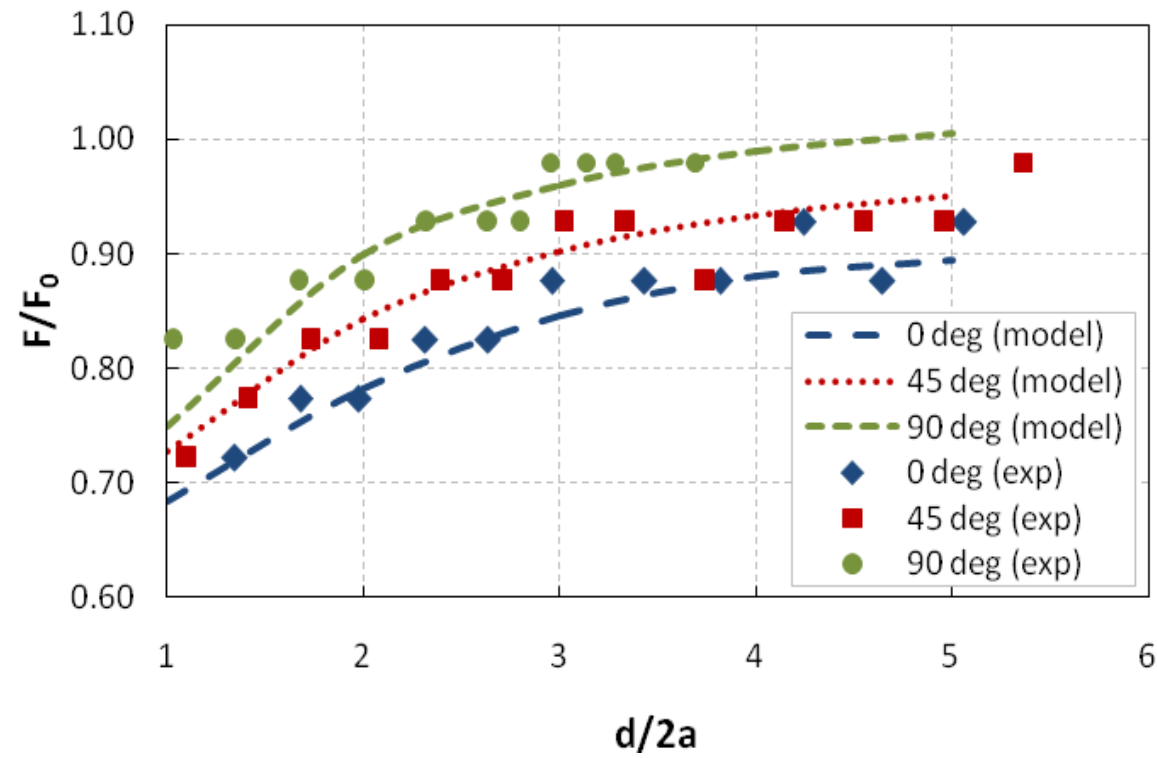

Figure 57: Experimental (points) and computational (lines) results for normalized drag force of the leading sphere as a function of normalized distance between the microspheres for 0, 45 and 90 degree angles. As the centerline goes from parallel to the flow to perpendicular to the flow, the drag force increases and reaches the undisturbed drag force faster. 


\subsubsection{FLUID VELOCITY AROUND TWO SPHERES PARALLEL TO THE FLOW}

Two polystyrene spheres were trapped in a uniform flow generated by the movement of the automated stage in the $x$ axis with $V_{\text {stage }}=350 \mu \mathrm{m} / \mathrm{s}$. Figure 58 a shows the measured velocity field in the vicinity of the trapped spheres (every third vector is shown for clarity). The leading sphere has a diameter of $28.5 \mu \mathrm{m}$ while the trailing sphere has a diameter of $26.6 \mu \mathrm{m}$. The spheres were set at a normalized distance $d / 2 a=$ 1.53 from each other. The measured velocity field is qualitatively and quantitatively consistent with the computationally predicted velocity field (Figure 58b), with a null velocity between the spheres.

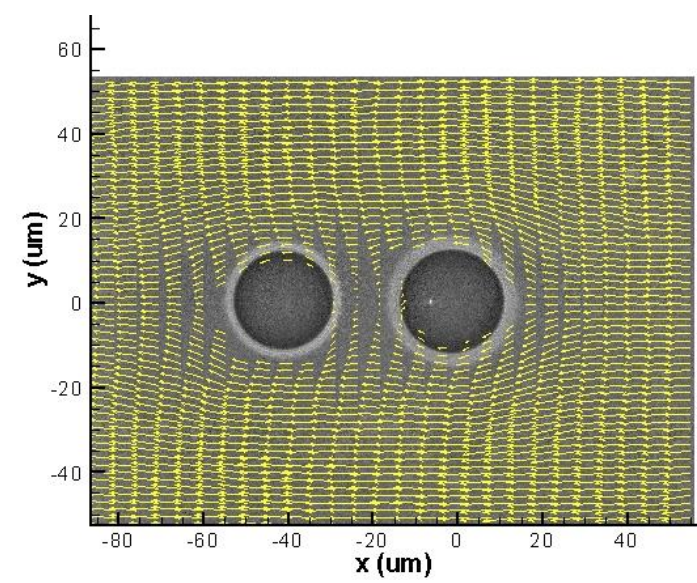

(a)

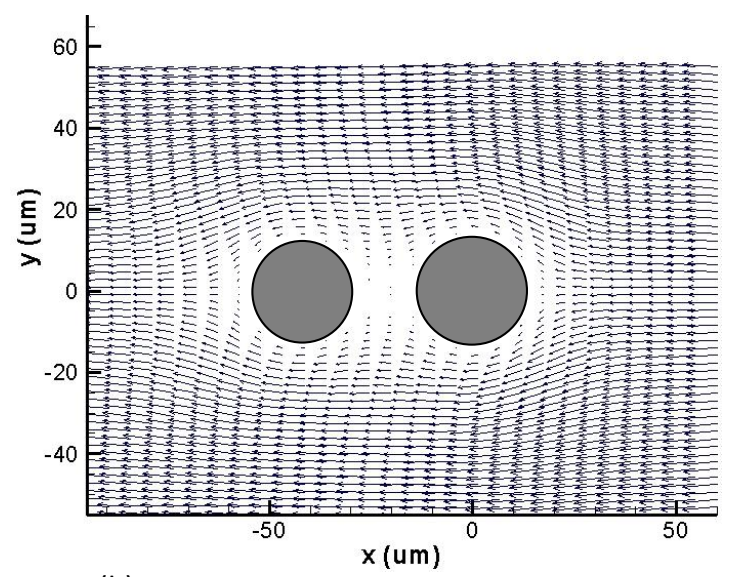

(b)

Figure 58: Comparison between the experimental velocity field and the computational prediction for two stationary spheres held at a normalized distance of 1.53 from each other in a uniform flow. (a) $\mu$ PIV 2-D velocity field around two trapped polystyrene microspheres (average diameter $2 a=27.6 \mu \mathrm{m}$ ) held in a uniform flow. (b) Model simulation of the experimental flow conditions. 
For quantitative comparison, Figure 59 shows the predicted and measured velocities along the inflow and outflow axes ( $x$ axis at $y=0$ and $z=50 \mu \mathrm{m}$ ). As expected, the $x$ direction velocity at the spheres surfaces is zero (no-penetration condition) and the velocity increases towards its free-stream velocity away from the spheres. Additionally, it is shown that at this separation distance between the spheres, the flow velocity is maintained at 0 between the spheres. Figure 59 shows strong quantitative agreement between the predicted and observed normalized velocity as a function of normalized distance $(x / 2 a)$ from the center of the leading sphere. The experimental results, however, show a small velocity between the two spheres.

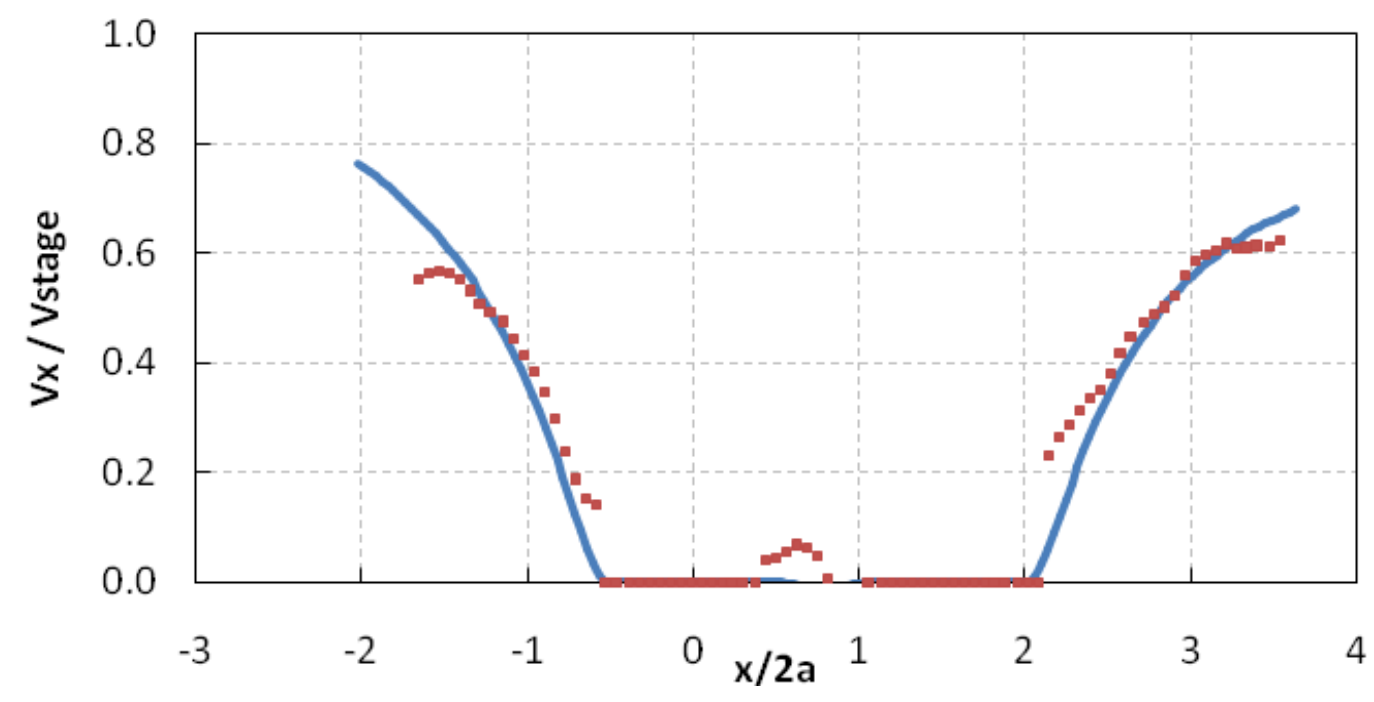

Figure 59: Comparison between measured (points) and predicted (line) velocities for two spheres

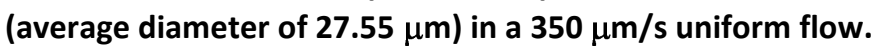




\subsection{DISCUSSION}

The experimental drag reduction on the leading sphere matched computational predictions. However the experimental drag reduction on the trailing sphere was higher than expected. This shift was observed to be systematic for all separation distances and flow angles tested and could be eliminated when decreasing the trap stiffness $10 \%$. The reason for this deviation is not clear. As stated the discrepancy may result from measurement error in the trap stiffness. However, during experiments, the spheres were initially positioned at the same height (focused at $50 \mu \mathrm{m}$ away from the bottom coverslip) in a quiescent fluid. It is observed that when the spheres are subjected to the uniform flow $(200 \mu \mathrm{m} / \mathrm{s})$, one sphere (trapped with Trap1, here the leading sphere) was systematically pushed away from the coverslip, and one sphere (trapped with trap 2, here the trailing sphere) lowered (Figure 60). This difference was estimated to provide a depth difference between the spheres of approximately $z=2$ to $3 \mu \mathrm{m}$.

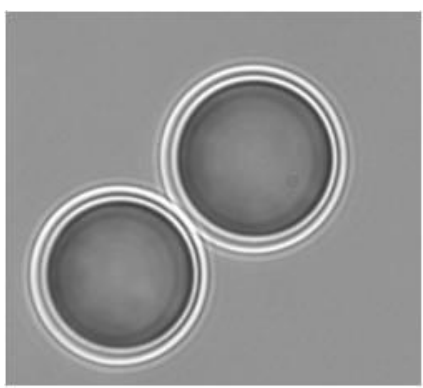

(a)

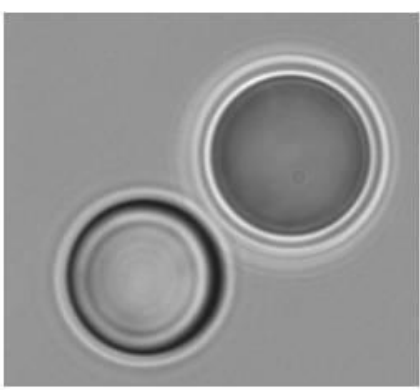

(b)

Figure 60: (a) Two spheres held at 45 degrees angle from horizontal axis in a stagnant flow. (b) The same spheres are shown when under a $200 \mu \mathrm{m} / \mathrm{s}$ uniform flow (from left to right). The leading sphere is shifted away from the coverslip while the trailing sphere is slightly shifted towards the coverslip. 
This shifting of positions could generate the systematic error for three reasons. First, the drag force decreases slightly for the sphere pushed away from the cover slip (the leading sphere) and increases for the sphere drawn towards the cover slip (the trailing sphere). With the displacement of 2-3 microns, this effect should be small, however it contributes to observed deviation. Second, we neglected the effect of one OT on the sphere trapped by the other OT. We assumed that the two spheres are within the focal plane and the trapping force is limited outside the trap focus. However the interaction between traps exists and has been extensively studied by Hough and Ou-Yang [Hough, 2002]. They examined the action of an oscillated trapped bead on another trapped bead placed in near proximity. They showed that for two traps with one trapping a bead and one "empty", the empty trap has an effect on the motion of the particle trapped for distances smaller than one-half of the particle radius. However optical interference between the two tweezers when both the particles are in the traps was shown to be insignificant. For our experiments, it is possible that the opposing trap affects the position of the other trapped sphere. As the difference in the vertical positions of spheres increases, the region of influence of the opposing trap increases rapidly. The actual interaction between the traps whether it is attraction or repulsion is unknown. Thirdly, the observed opposite shift in the vertical positions of the spheres increases the complexity of the flow. The assumption of a planar flow in the theoretical and 
computational models is no longer valid. The effects of this 3-D interaction coupled with the bottom cover slip needs to be explored.

In the computational and theoretical models, the two spheres are modeled as fixed rigid spheres with no-slip at the sphere surface. During experiments it is observed that for any angle other than $0^{\circ}$ the spheres rotate in the uniform flow. This unplanned observation develops from optical variations within the polystyrene spheres and the collimated back lighting. The rotation rate increased with decreasing sphere separations and increasing flow angles (Figure 61). The rotation of the two spheres induces recirculation patterns in the flow field.

Figure 62 provides a schematic of the observed flow patterns. The effects of the sphere rotation on fluid drag need to be explored. Currently, theoretical models do not exist and computational models with free rotation of the spheres are not available in the Microscale Laboratory.

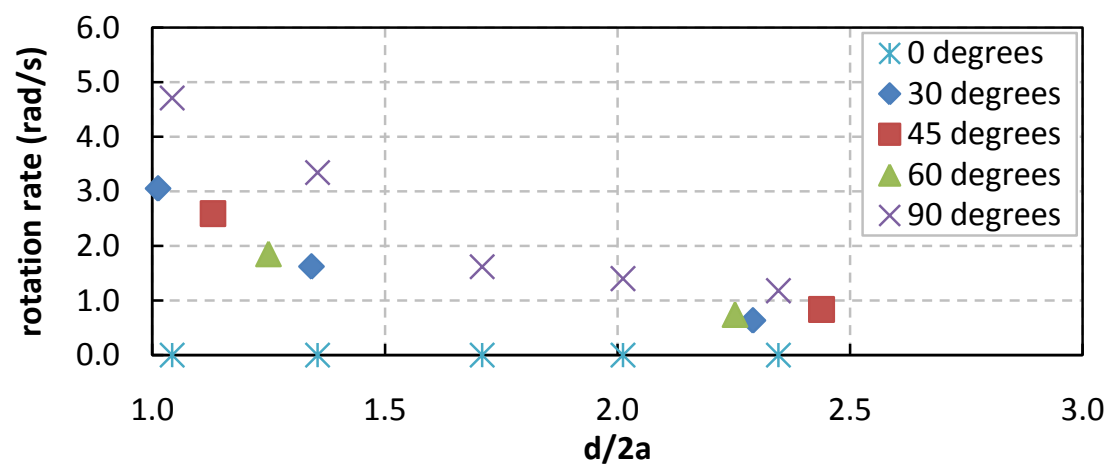

Figure 61: Spheres rotation rate as a function of distance between them for different angles $\theta$. 


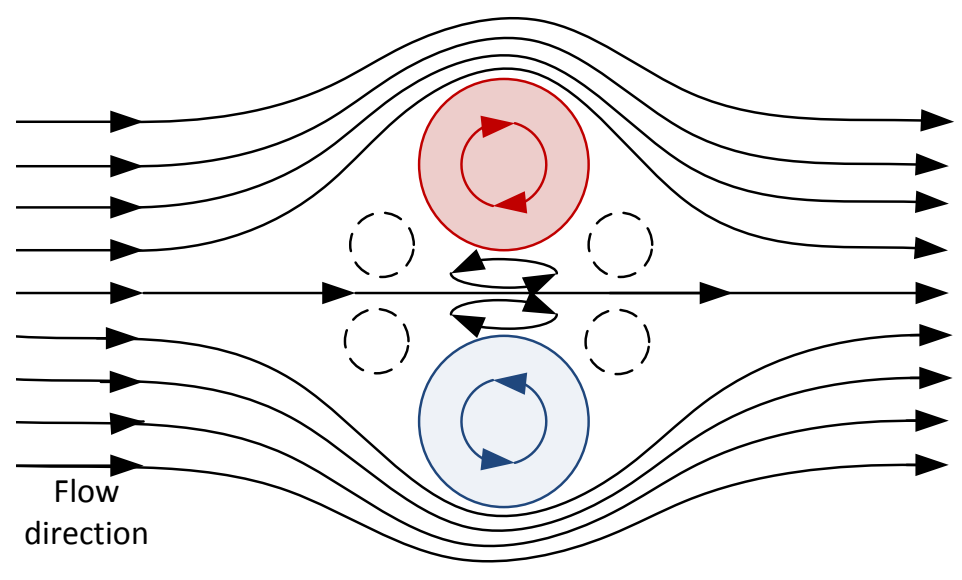

Figure 62: Rotational movements of the two spheres for $\theta=90^{\circ}$. This movement induces recirculation areas and stagnation points (dotted circles) that are not observed in the computational model.

\subsection{SUMMARY}

The flow velocity around two optically suspended spheres in a uniform flow and the resulting drag reduction from hydrodynamic interactions was studied. The spheres were positioned with varying separations and centerline angles relative to the inflow axis. The experimental results qualitatively agreed with the computational and theoretical predictions, however quantitatively, theory predicted the largest drag reduction. The experimental and computational results agreed quantitatively well for the $\mu$ PIV velocity measurements and the predicted velocity field, as well as for the drag reduction of the leading sphere. However, the trailing sphere drag reduction was significantly less than both the leading sphere experimental results and the computational predictions. This shift appeared to be systematic for all the distances and angles tested and could be eliminated by decreasing the trap stiffness $10 \%$. The 
reason for this deviation is unclear. This discrepancy may result from measurement errors associated with calibration of the trailing sphere optical trap or may develop as a result of out of plane displacements which develop upon initiation of the uniform flow. Further experiments with automated position monitoring and on-the-fly depth adjusted are necessary to fully resolve this issue. An interesting observation that must be explored is the apparent free rotation of the spheres. The effects of free rotation on the theoretical and computational models need to be addressed. 


\section{CHAPTER 7}

BIOLOGICAL CELL EXPERIMENTS

In the United States, osteoarthritis affects over twenty million people, a number predicted to double in the next twenty years. Healthy chondrocytes and osteoblasts experience multiple stress states resulting from hydrostatic, hydrodynamic, compressive, tensile, and shear forces that maintain the phenotype and production of new tissue. Biomechanical factors such as excessive repetitive loading may negatively influence cartilage and bone cell behavior leading to pathological matrix synthesis and increased tissue degradation. However, optimum mechanical conditions are not completely known. Moreover, the process of mechanotransduction, which transforms the mechanical environment experienced by cells into a biomolecular response, has not been fully characterized at the tissue or cell level.

The promise and contribution of biomechanics is to advantageously control cell function in the treatment of disease or in regenerative medicine [Kamm, 2004]. Exploring biomechanics at the cellular level is now becoming feasible thanks to the advances in technology. A number of techniques exist to characterize cell membrane and cytoplasm mechanics (see Chapter 1 for the complete range of techniques). Micropipette aspiration applies a negative pressure to the cell for localized membrane stretching with results reported for red and white blood cells [Bull, 1983], [Hochmuth, 
1987][Evans, 1989], endothelial cells [Sato, 1987], as well as chondrocytes [Jones, 1999]. Cytoindentation incorporates a probe (such as a $2 \mu \mathrm{m}$ diameter glass microfiber) that compressively loads a cell adhered to a surface[Petersen, 1982][Pasternak, 1985][Felder, 1990]. Atomic Force Microscopy (AFM) manipulates a cantilevered probe (tip radius ranging from a few nanometers to a few micrometers) for tension or compression loading [Hassan, 1998][Rotsch, 2000][Radmacher, 2002]. A slightly larger version of the AFM technique facilitates microscale indentation of an entire cell [Koay, 2003][Leipzig, 2005]. Microplates, either rigid or flexible, can apply a range of mechanical stresses to an entire cell including tension or compression [Thoumine, 1999]. Other than the micropipette technique, most evaluations of single cells or groups of cells require surface attachments to provide a reaction force. These surface adhesions constrain the cell and may or may not involve additional cytoskeletal manipulation such as the binding of integrins to ligands. This compounds the cellular mechanical response.

Contact-free cell deformation applying innovative techniques has been explored to a lesser extent. One of the oldest techniques, the rheoscope [Schmid-Schonbein, 1969] examined red blood cells by measuring blood viscosity as a function of cell deformation and cell aggregation. Later, using the rheoscope as well as an ektacytometer, Bull et al.[Bull, 1983] studied the elliptocytic red cells deformability under different shear stresses. The appearance of optical tweezers and derived 
technology in the late 1980's opened the door to new testing of cells without physical contact. In the optical channel, Kaneta et al. [Kaneta, 2001] elongated red blood cells passing through a focused beam by hydrodynamic stresses. In the optical stretcher, Guck et al. [Guck, 2001] held and stretched a cell optically. Because the beam is not focused, higher powers can be applied to manipulate the cell without damaging it.

A partial motivation in developing the $\mu$ PIVOT was to characterize multiaxial and multimodal cellular biomechanics. The combination of $\mu$ PIV and OT provides a unique platform for controlling and monitoring cellular biomechanics (stress and strain) as a precursor to deciphering mechanobiology. As an enhancement to the $\mu$ PIVOT, microfluidics provides additional control of the local fluidic microenvironment including applied shear and normal stresses. In this chapter, we describe the integration of the $\mu$ PIVOT with microfluidics and present results of this new approach for threedimensional (3-D) mechanical manipulation of single cells. The study examines the viability of chondroblasts under optical tweezers, analyzes cells in uniform flows calculating the deformation and trap stiffness, verifies the accuracy of cell shape assumptions through computational modeling, identifies the maximum fluid induced stresses possible in representative uniform and extensional flows, and compares the deformation characteristics of osteoblasts and myoblasts. The overall objective of this work is to outline a method to explore individual cellular biomechanics. 


\subsection{MATERIALS AND METHODS}

\subsubsection{CELL CULTURE}

In collaboration with the Oregon Health \& Science University (OHSU), primary cultures of chondrogenic and osteogenic tissues were generated from rat long bones. We followed the procedures and protocols for bone and cartilage cell isolation described in Jones [Jones, 1996]. Further information on isolation, proliferation, and differentiation of osteoblastic cells can be found in Declercq et al [Declercq, 2004]. The muscle cells used in the present studies were the mouse derived myoblast $\mathrm{C} 2 \mathrm{C} 12$ cell line obtained from ATCC (CRL-1772; Manassas, VA). Cells were cultured in tissue culture flasks using $\alpha$-MEM (Cellgro, Herndon, VA) supplemented with $10 \%$ fetal bovine serum (GIBCO, Gaithersburg, MD), 2\% glutamine, 1-2\% penicillin-streptomycin (Cellgro), and 2\% $1 \mathrm{M} \mathrm{HEPES}$ under standard culture conditions [Jaasma, 2007] $\left(37^{\circ} \mathrm{C}, 5 \% \mathrm{CO}_{2}, \mathrm{pH}=7\right)$. Cells were cultured up to 4 passages for all experiments. PO (= passage 0$)$ was the batch of cells directly harvested from the cartilage tissue and consisted mainly of chondrocytes (rounded cells) and chondroblasts (attached). Osteogenic cultures exhibited firmly attached osteoblasts with a typical epithelial morphology. Figure 63 shows pictures of bone (left) and cartilage cells (right) when attached to the culture flask before processing. Note, cells spread on the surface and are well attached. The bone cells (left picture) are recognizable by their cobblestone appearance and white halo around the cells which corresponds to gap junctions between the cells. 

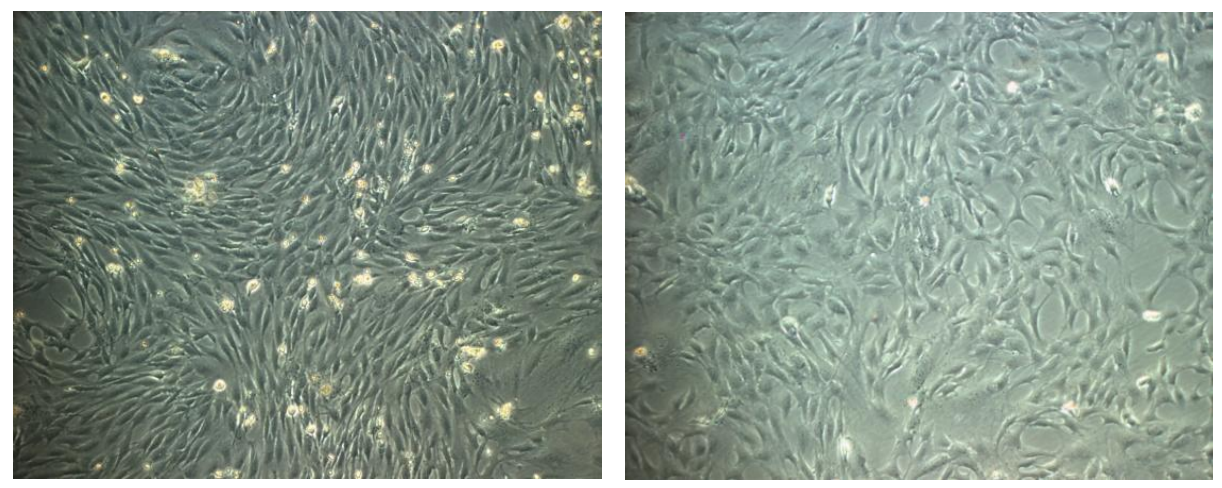

Figure 63: Attached cells: on the left osteoblasts, on the right chondroblasts (P1). Cells are approximately $15 \mu \mathrm{m}$ in diameter.

The bright white spots indicate the beginning of mineralization. The cartilage cells (right picture) are usually less elongated and are not organized like the bone cells.

Myoblasts were firmly attached elongated cells that retained the ability to form myotubes when exposed to tissue culture medium with reduced serum content (2\%). Cells from older than 4 passages were not used due to their ability to de-differentiate. On the day of experimentation, cells were detached from the flask surface with $0.05 \%$ trypsin-EDTA (Ethylenediamine tetraacetic acid, GIBCO). Harvested cells were diluted $(10,000$ cells $/ \mathrm{mL})$ in a solution containing $50 \mathrm{~mL}$ of artificial cerebrospinal fluid, $1 \mathrm{~mL}$ of HypoThermosol FRS (BioLife Solutions, Bothell, WA), and one micro-molar of EDTA to avoid clustering. Cells were examined at room temperature for all experiments $\left(\sim 20.5^{\circ} \mathrm{C}\right)$. The flow media consisted of a physiological buffer $(127 \mathrm{mM} \mathrm{NaCl}, 5 \mathrm{mM} \mathrm{KCl}, 2$

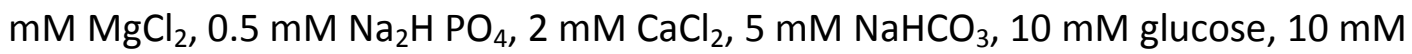
HEPES, 0.1\% BSA adjusted to $\mathrm{pH} 7.4$ ). 


\subsubsection{CELL RESERVOIRS AND MICROFLUIDIC DEVICE FABRICATION}

To test cells under various mechanical stimuli such as local or global stresses in static or dynamic environments, multiple microfluidic devices were created. To study cell responses to local OT-induced stresses and global hydrodynamic stresses of uniform flows, a simple rectangular reservoir was constructed. These simple reservoirs were modified slightly to investigate the impact of hydrostatic pressure on the cells by adding variable height input and exit ports. To study cells subjected to more complex flows (such as an extensional flow), microfluidic chips were designed and fabricated. In each device, an isolated cell is suspended by the optical tweezers without attachment to any surface or mechanical restraint.

\subsubsection{Cell reservoirs}

Figure 64(a) provides an image of a simple cell reservoir defined by four walls of double-face tape (foam mounting tape of approximately $1 \mathrm{~mm}$ thick) attached to a coverslip. The reservoir is filled with a solution of diluted cells $(10,000$ cells $/ \mathrm{mL})$ and enclosed by a second coverslip. The reservoir was then placed on the microscope automated stage that was either stationary for static experiments or moving at constant velocity to generate a uniform flow field around a suspended cell held at a fixed position. 

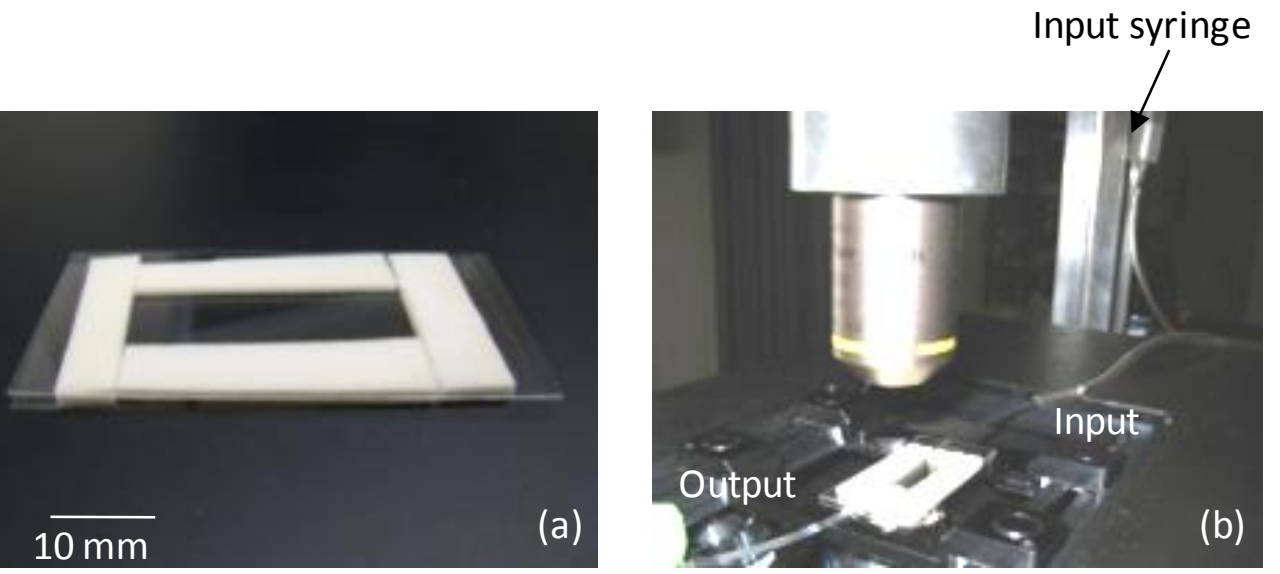

Figure 64: Microfluidic chip design (a) Cell reservoir used for static experiments and uniform flow experiments (30 $\mathrm{mm} \times 10 \mathrm{~mm}$ ). (b) Cell reservoir used for hydrostatic experiments. The input is set at different heights while the output is closed.

To investigate the effects of hydrostatic pressure, the reservoir was modified to include variable height input and output ports which consist of Tygon tubing (Fisher Scientific, Pittsburgh, PA) sealed to the reservoir with Epoxy. The input tubing was connected to an open syringe (no plunger) attached to a vertical micrometer for fluid height displacement (Figure 64(b)).

\subsubsection{Microfluidic chip}

Figure 65 shows the silicone mold of a current cross-junction channel. The channel dimensions were $500 \mu \mathrm{m}$ wide by $50 \mu \mathrm{m}$ deep. The geometry creates an extensional flow environment with a stagnation point at the cross-junction geometric center. A cell may be positioned at this point and subjected to hydrodynamic stresses without a net drag force. 
Two iterations of the cross-junction design were initially fabricated. The cell reservoir design was eliminated because static cells tended to cluster and stick to the walls of the reservoir after approximately 20 minutes. Figure 66 shows the first version of the cell chip.

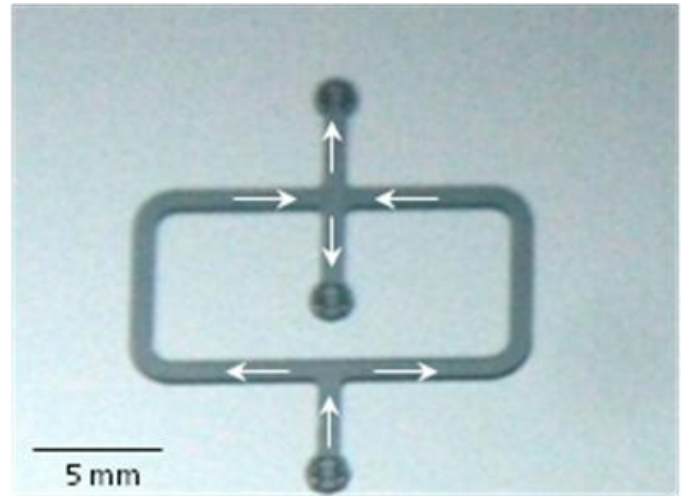

Figure 65: Silicone mold of the cross-junction channel. The channels are $50 \mu \mathrm{m}$ deep $\times 500 \mu \mathrm{m}$ wide. White arrows show the directions of the flow.
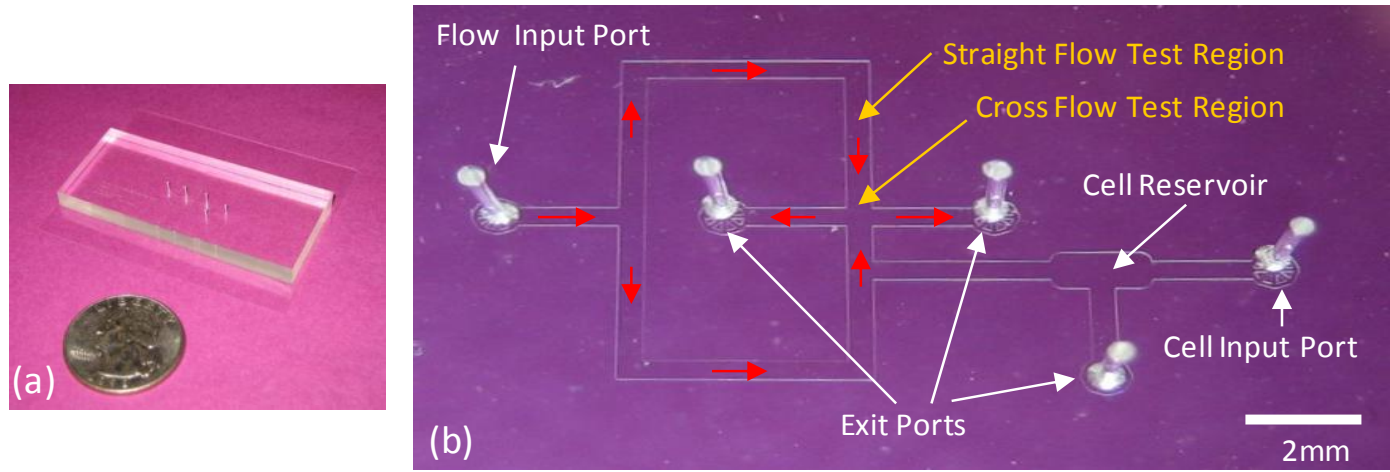

Figure 66: (a) Microfluidic chip of the cross-junction. (b) Detailed picture of the first version of the crossjunction channel. The red arrows represent the directions of the flow. 
Both chips were fabricated using a standard soft lithography approach with polydimethylsiloxane (PDMS) (Sylgard 184, Dow Corning, Midland, MI) as the soft peeled material. The PDMS chip was bonded to a cover slip, after having punched 20 gauge holes ( $0.9 \mathrm{~mm}$ ) for microport access. A gravity driven flow was generated by simply attaching input and output open syringes placed at different heights. The output syringe height was held constant while the input syringe height was controlled in order to vary the flow rate. Velocities up to $750 \mu \mathrm{m} / \mathrm{s}$ were produced by fluid heads of $2.5 \mathrm{~cm}$. The syringes were connected to the microfluidic chip via Tygon tubing (Fisher Scientific, Pittsburg, PA) attached to metal pins (23 gage, $0.025^{\prime \prime}$ OD $\times 0.017$ " ID, Stainless steel type 304, New England Small Tubes Corp., Litchfield, NH) that were directly inserted to each of the entry/exit ports of the microchannel. The resulting microfluidic chip arrangement allowed for a controllable, constant, and steady flow.

\subsection{EXPERIMENTAL PROTOCOLS}

\subsubsection{STATIC ENVIRONMENT: OT-INDUCED STRESS AND HYDROSTATIC-INDUCED STRESS}

This study has explored local and global stresses applied to cells in a static environment. Local stresses were also applied directly using the dual OT. Global stresses were induced hydrostatically. 
For local stress experiments, a low concentration of 10,000 cells $/ \mathrm{mL}$ was placed in the rectangular reservoir. A cell was trapped with the dual trapping beams (an equally split laser) and positioned a few microns away from the coverslip surface. A range of laser powers ( $30 \mathrm{~mW}$ up to $1 \mathrm{~W}$ at the sample) was applied to the cells while their viability was monitored through Trypan blue absorption (1:1 volume ratio) added to the culture media. For stretching of the cell with two optical traps, one trap remains fixed while the second one is slowly directed away from the center of the cell. Trap movement is controlled by positioning lenses located on the automated vertical and linear stages (MVN80 and UMR8.25, Newport). As stated earlier, the stage translation range of $25 \mathrm{~mm} \times 25 \mathrm{~mm} \times 12.5 \mathrm{~mm}$ provided trap movements in excess of the field of view.

Due to its ease of application and the homogeneous stress environment, hydrostatic pressure was applied to the cell for global static stress measurements. In these experiments, a low concentration of cells $(10,000$ cells $/ \mathrm{mL})$ solution was placed in the input syringe. The output tubing was opened to air and maintained at the reservoir height while the input syringe was slightly raised to fill the reservoir. After elimination of all air bubbles in the system, the output tubing was clamped to halt the flow and a static environment was obtained. A cell was then trapped a few microns away from the coverslip at the minimum power (approximately $30 \mathrm{~mW}$ at the sample) to position and maintain the cell in focus. Hydrostatic pressure was varied by adjusting the input 
syringe height relative to the reservoir. A maximum syringe height of $10 \mathrm{~cm}$ was applied.

\subsubsection{DYNAMIC ENVIRONMENT: UNIFORM AND EXTENSIONAL FLOWS}

To apply hydrodynamic forces, a simple uniform flow was generated by trapping a cell and moving the automated stage at a constant velocity. Cells were trapped at the minimum laser power ( $30 \mathrm{~mW}$ at the sample) to minimize potential radiation damage to the cell.

Under uniform flow conditions, the cell undergoes two main forces: the applied drag force $\left(F_{d r a g}\right)$, due to hydrodynamic stresses on the cell and the reacting trap force $\left(F_{\text {trap }}\right)$. For statically stable trapping, these forces are equal in amplitude and opposite in direction. From the experiments, two parameters can be determined: 1) the cell deformation $\left(D_{12}\right)$; and 2 ) the trap stiffness $(k) . D_{12}$, the Taylor deformation parameter, is computed by measuring the major and minor axes of the cell ( $L$ and $B$ respectively), such that $D_{12}=(L-B) /(L+B)$. The axes were measured using the NIH open software ImageJ. An ellipse aligned with the flow direction was superimposed on the cell image to fit the general outline of the cell boundary. The axes of the ellipse were recorded and taken as the minor and major axes. If the cell is smooth, sub-pixel $(<0.125 \mu \mathrm{m})$ accuracy in the axes length or position can be achieved. However, since the cell surface may be rough, the ellipse axis resolution with this method is $+/-2$ pixels $(0.250 \mu \mathrm{m})$. The trap 
stiffness, $k$, was calculated by equating the drag force to the trap force, $F_{\text {drag }}=F_{\text {trap, }}$ using the same protocol as the one used for the polystyrene spheres experiments. As described in Chapter $5, F_{\text {trap }}$ is equal to $k . \Delta x$ for small displacements (the linear regime) where $\Delta x$ is the difference between the cell position (geometric center) when trapped without flow and trapped with flow. Figure 67 shows a typical series of pictures taken with one cell. The cell was first imaged at zero flow velocity to obtain its position when no force (apart from the OT force) is applied to it. It was then photographed at several stage velocities.
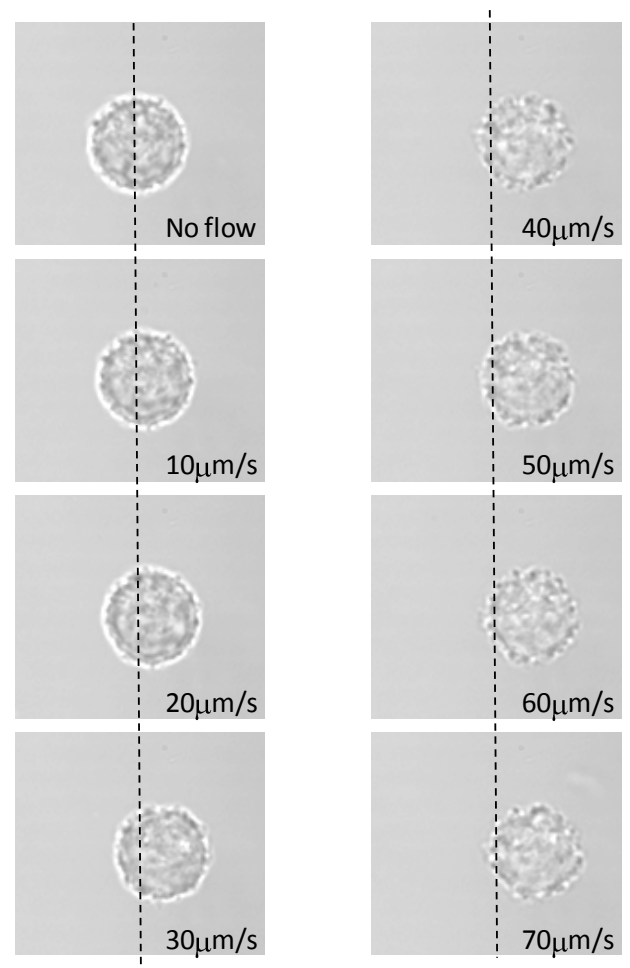

Figure 67: Pictures of a $17 \mu \mathrm{m}$ diameter chondroblast trapped with OT and undergoing different uniform flow velocities. The cell is shifted to the right due to the force of the flow coming from the left. The vertical line indicates the center of the cell when in a static environment. 
The uniform steady flow velocities are $10,20,30,40,50,60$, and $70 \mu \mathrm{m} / \mathrm{s}$ left to right. Only the $x$-position of the cell varies. This position was measured for five images of each flow velocity with the resulting average applied for better accuracy. To measure position, two software packages were used; an image manipulation program, GIMP 2.6.6, for a pre-processing of the image, where the image background is eliminated, and an object tracking software, Spotlight-8, NASA, to determine the cell's position. Figure 68 shows the basic steps to perform this task. The background is first eliminated and the image is then converted into a black and white image using a Threshold tool. The cell has to be represented as a whole object, so any "holes" are filled to obtain a uniformly white object. Finally, the center tracking tool provides the position of the cell. The cell's position difference with the no flow-position can now be calculated for each flow velocity. Finally, drawing a chart of the drag force (function of flow velocity, as mentioned below) as a function of cell displacement, we can extract the value of the trap stiffness (slope of the line) for a particular cell.

Note, we only calculate $k$ for the linear regime where the displacement as a function of drag force has a linear fit of $R^{2}>0.9$.
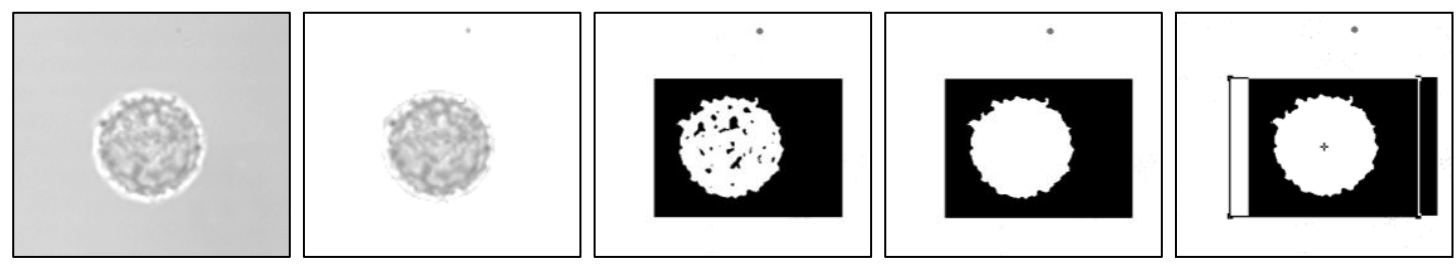

Figure 68: Image processing steps to determine the cell's position. 
Since cell deformation is sufficiently small (cell protrusions are negligible as well) in this study, we can assume the cell is spherical thus $F_{\text {drag }}=F_{\text {stokes }} / C$, where $F_{\text {stokes }}=6 \pi \mu a v_{\infty}$ (Stokes drag), $\mathrm{C}$ is a correction factor which accounts for particle-wall effects associated with the presence of the bottom coverslip, $\mu$ is the fluid dynamic viscosity, $v_{\infty}$ is the fluid velocity away from the sphere, and $a$ is the radius of the cell. Following Faxen's law, the correction factor [Happel, 1983], C, is equal to $1-(9 / 16)(a / l)+(1 / 8)(a / /)^{3}-$ $(45 / 256)(a / /)^{4}-(1 / 16)(a / l)^{5}$ where $/$ is the distance of the cell from the bottom coverslip. The distance, I, was determined by calculating the difference between the objective lens position (precision $\sim 0.1 \mu \mathrm{m}$ ) when a cell was trapped during the experiment and the objective lens position at the coverslip (determined by both reflection of the optical trap at the coverslip and by focusing on a cell resting at the coverslip and subtracting the cell radius from the objective lens position). A factor of 0.878 is applied to account for the focal shift due to the index of refraction mismatch between the culture media $\left(n_{\mathrm{f}}=1.33\right)$ and the coverslip/immersion oil $\left(\mathrm{n}_{\mathrm{g}}=1.515\right)$. The top coverslip does not need to be considered in the calculations. Its effect is negligible (C $>0.99$ ) as it is approximately $1 \mathrm{~mm}$ away from the bottom coverslip and thus several hundreds of microns away from the cell. Note, in the above calculations, the effects of possible spherical aberrations due to the index of refraction mismatch between the culture media $\left(\mathrm{n}_{\mathrm{f}}=1.33\right)$ and the coverslip/immersion oil $\left(\mathrm{n}_{\mathrm{g}}=1.515\right)$ on trap stiffness measurements were not taken into consideration because the effect is inconsistent as 
demonstrated in the studies by Fallman and Axner [Fallman, 2003] and Im et al., 2003 [Im, 2003]. Regardless, to minimize errors that could be introduced due to the difference of the trap behavior with sample depth and optical aberrations, all cell and calibration experiments in uniform flow were conducted at the same distance from the coverslip.

A limiting factor in the uniform flow experiments is the maximum hydrodynamic stress that can be applied to a cell. As the velocity increases, so do hydrodynamic stresses and fluid drag. To overcome this increase in drag and maintain a stable trapped cell, the trap power must be increased. This may lead to potential damage of the cell. In order to increase the hydrodynamic stress applied to the cell and alleviate potential radiation damage, fluid flows which generate no net drag are required.

Planar extensional flow has been used extensively in the study of drop deformation and breakup [Tretheway, 2001], [Bentley, 1986a], [Bentley, 1986b]. This symmetric flow contains a stagnation point. A symmetric particle centered at the stagnation point experiences no net drag. In this work we created a pseudo-planar extensional flow in a microfluidic cross junction. Microfluidic cross junctions have mainly been used for droplet generation[Wu, 2008][Yang, 2007] and combining fluid flows. The flow arrangement for droplet generation consists of three inlets (usually, the two opposite inlets introducing oil, and the third inlet being an immiscible fluid from which the droplets are formed), and one outlet, where droplet formation occurs. However, in 139 
our experiments we apply a cross-junction to generate fluid compression along the two opposing fluid inlets and fluid extension along the fluid outlets. The components of the fluid velocity $\left(v_{x}, v_{y}\right)$ in the directions of the extensional and compressional axes vary linearly with position such that $v_{x}=\gamma \cdot x$ and $v_{y}=\gamma \cdot y$, where $\gamma$ is the shear strain rate, $x$ is the distance from the stagnation point along the extensional axis and $y$ is the distance from the stagnation point along the compressional axis. The bottom picture in Figure 69 shows $\mu$ PIV velocity data for fluid flow in a cross junction. When a cell is held stationary at the stagnation point, it experiences compressive and tensile stresses whose magnitude depends on the flow rate (see Chapter 8). Integration of these stresses around the cell yields no net drag force regardless of flow rate.

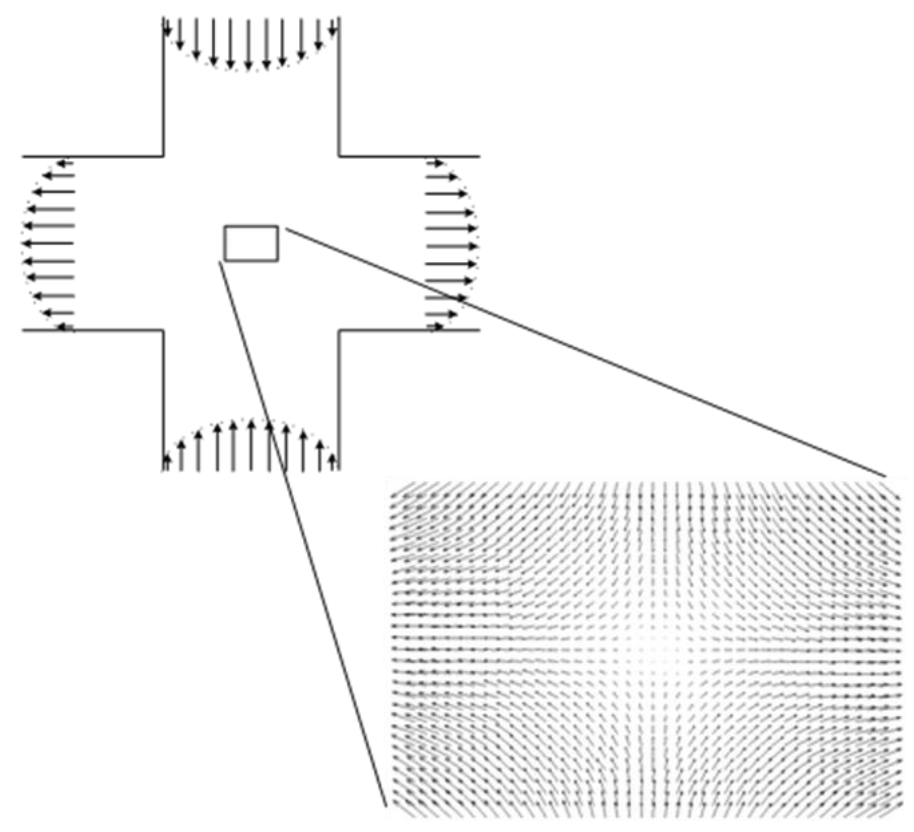

Figure 69: Sketch of a pseudo-planar extensional flow. The bottom picture is the undisturbed velocity field of a cross-junction flow at the stagnation point. Velocities are measured with our $\mu$ PIV system. 
To apply an extensional flow on a cell, the cross-junction microfluidic chip is first primed by inserting a low concentration of cells $(10,000$ cells $/ \mathrm{mL})$ through the upstream tubing. The low cell concentration is high enough to locate and trap a cell but sufficiently dilute to minimize flow disturbances from the remaining untrapped cells and to maintain Newtonian fluid behavior. A single cell is chosen and followed by moving the microscope translation stage at the same velocity and direction as the flow until it is close to the stagnation point. The cell is then trapped (by turning one OT laser on and shuttering the other one) and placed at mid-width and mid-height of the channel in order to avoid any cell rotation due to the velocity gradients across the channel. The hydrodynamic pressure and fluid flow is finally increased by slowly continuously raising the input syringe. The use of gravity driven flow provides a very smooth flow with no oscillation and no perturbation on the stagnation point location. Variations of the hydrostatic head provide two effects: variation of hydrostatic pressure and flow rate. CCD images of cells are recorded at known input and output syringe heights to monitor cell behavior and determine cell deformation. All the experiments were performed at low powers ( $30 \mathrm{~mW})$ and for a short period of time (less than 4 minutes).

\subsubsection{COMPUTATIONAL MODELING}

In order to verify the assumptions of the spherical cell shape in the uniform flow drag force calculations, different models mimicking the cell shape were designed using the multiphysics modeling software COMSOL Multiphysics ${ }^{\circledR}$ version 3.5a (COMSOL Inc., 
Burlington, MA). For each model, the drag force was extracted and compared to the hypothesized drag force for a spherical object of the same size.

For the experimental conditions (microscale, low velocities), the flow is laminar $\left(\operatorname{Re}<10^{-3}\right)$ and steady. The fluid was modeled as an incompressible Newtonian fluid with a density $\left(1000 \mathrm{~kg} / \mathrm{m}^{3}\right)$ and viscosity $\left(10^{-3} \mathrm{Ns} / \mathrm{m}^{2}\right)$ equal to that of the culture medium at room temperature. Following the described experimental setup, the global shape of the object was first altered with increasingly oval shapes to up to $50 \%$ elongation in each of the three directions $(x, y, z)$ while maintaining the object volume (Figure 70$)$. The volume set corresponds to a $20 \mu \mathrm{m}$ diameter sphere.

Then, protrusions were added to a spherical object with its core measuring 20 $\mu \mathrm{m}$ in diameter. Among all the cells tested, we have observed different categories of protrusions.

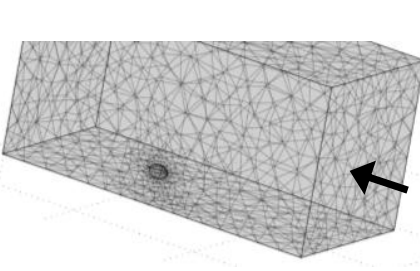

(a)

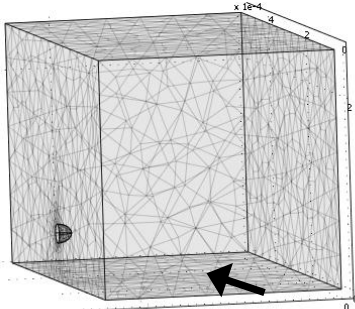

(b)

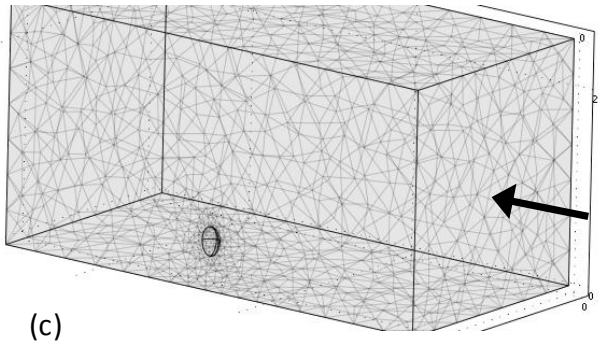

(c)

Figure 70: A 3-D representation of the computational flow half-space domain for uniform flow around a: (a) $50 \%$ elongated sphere in the x-direction (flow direction). (b) $\mathbf{5 0 \%}$ elongated sphere in the $y$ direction. (c) $50 \%$ elongated sphere in the z-direction. The arrows indicate the direction of the flow. 
They could be small (length of a few percent of the radius of the cell) to very long (up to 1.5 times the radius of the cell) and varying in number ( 0 to approximately 20). The average number of protrusions was calculated to be 4 protrusions, among the $50+$ cells tested. We also observed that protrusions are usually smaller when they are more numerous. We model several scenarios with various protrusions (size and number). The protrusions were first set to be 1.5 times the cell radius in length $(15 \mu \mathrm{m})$ from the cell surface. They were designed as cones measuring $25 \mu \mathrm{m}$ in length from their base (at the center of the cell) with a base radius of $1 \mu \mathrm{m}$ and a semi-angle equal to 1 . First, only one protrusion was designed in either the $\mathrm{x}, \mathrm{y}$ or $\mathrm{z}$ direction. For the $\mathrm{x}$ direction, the protrusion was placed either facing the inflow or facing the outflow. Four protrusions were then distributed on the cell, one in each direction. Six protrusions were then examined along each axis direction (Figure 71).

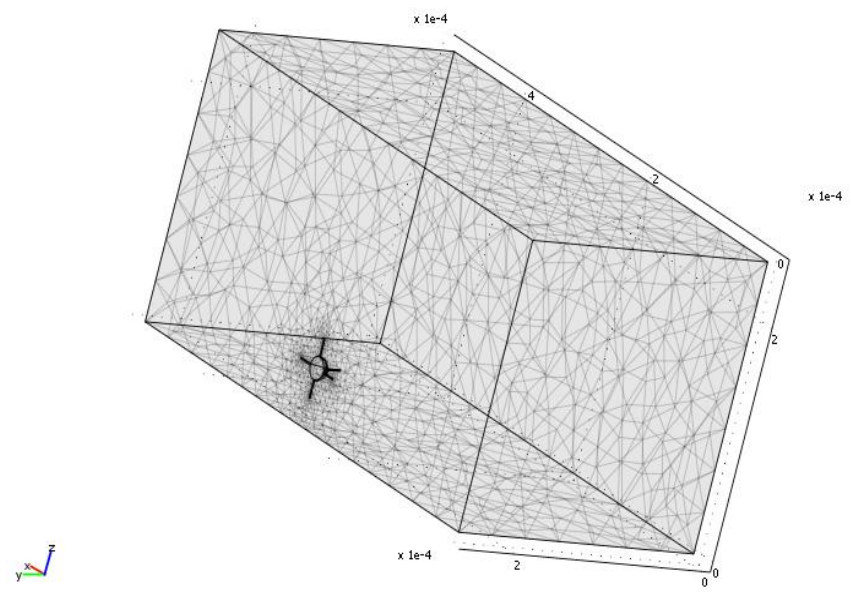

Figure 71: Modeling of the cell with six long protrusions. 
Finally, 26 small protrusions ( $2 \mu \mathrm{m}$ from the cell surface) were placed symmetrically across the entire cell (Figure 72).

The flow domain is a rectangular parallelepiped of dimensions $x=500 \mu \mathrm{m}, \mathrm{y}=250$ $\mu \mathrm{m}$, and $z=250 \mu \mathrm{m}$. It represents half of the region of interest as a symmetry plane $(x-z$ plane) slices the whole domain in half to reduce computational effort. For all of these models, the half-object (cell model) was placed $50 \mu \mathrm{m}$ from the bottom coverslip (zdirection), and half way in the x-direction. The bottom coverslip is modeled as a no-slip wall boundary moving at a constant velocity of $100 \mu \mathrm{m} / \mathrm{s}$. The opposite side and top boundaries are also modeled as no-slip walls moving at $100 \mu \mathrm{m} / \mathrm{s}$.

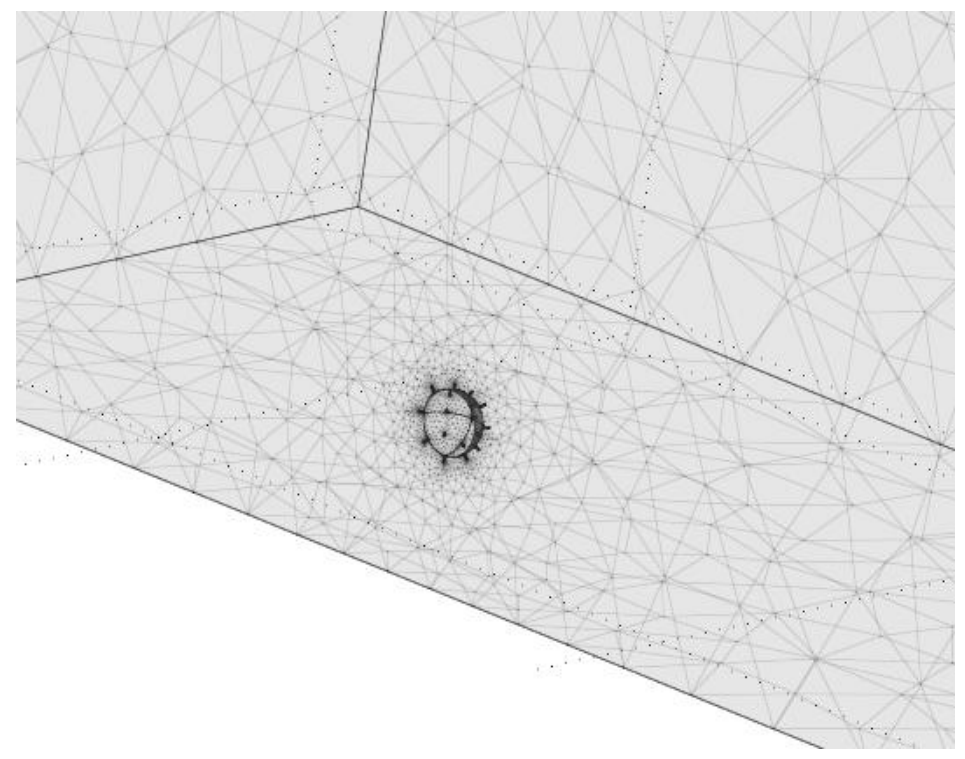

Figure 72: Modeling of the cell with twenty-six small protrusions. 
The inlet flow is prescribed as a uniform velocity (same value as the walls) and the outlet is an open boundary with no viscous stress and the pressure set to 0 . For each of the models, the computational domain was meshed with more than 10500 polyhedrals to provide a numerical resolution greater than experimental measurements. A refined mesh was set near the cell surface.

COMSOL solves the coupled steady state incompressible Navier-Stokes equation and the mass continuity equation iteratively.

$$
\begin{aligned}
& \rho\left(\frac{\partial v}{\partial t}+\boldsymbol{v} * \nabla v\right)=-\nabla p+\mu \nabla^{2} \boldsymbol{v}+\mathbf{f} \\
& \nabla * \boldsymbol{v}=0
\end{aligned}
$$

where $\rho$ is the fluid density, $v$ is the fluid velocity vector, $t$ is time, $\nabla p$ is the pressure gradient, $\mu$ is the fluid viscosity, and $\mathbf{f}$ is an added vector representing other forces applied to the fluid. The additional body force term, $\mathbf{f}$ is zero for this work.

\subsubsection{STATISTICS}

The trap stiffness $(k)$ of an optical trap is known to not only depend on the laser properties but also on the trapped object. It varies with the object's size and shape as well as the index of refraction and other material properties. The hypothesis for our experiments here (measurement of trap stiffness on cells) was that the $\mu$ PIVOT could differentiate normally indistinguishable cells. For example, we could distinguish 
chondroblasts from the different zones of cartilage, different passages, or diseased versus healthy cells.

More than fifty cells were tested and their trap stiffness calculated. The One-way Analysis of Variance (ANOVA) Single factor was used to compare the trap stiffness acquired for cells that differ in type (chondrocyte or osteoblast), passage (P1, P2 or P3), surface roughness (values from 1 to 5 were assessed with 1 being very smooth and 5 being very granular), time between experiment and trypsinization, number of cilia, size of cilia, and cell concentration during transport from extraction laboratory (OHSU) to experiments laboratory (Microscale Laboratory, PSU). The P-value used was set to 0.05 .

Two-way ANOVA studies would have been interesting to discover if there was any trap stiffness difference for paired variables. However, this test was not possible. While each group had different sizes which we could account for, not all the groups were populated.

\subsection{RESULTS AND DISCUSSION}

Coupled with microfluidics, the $\mu$ PIVOT provides a unique ability to subject the same individual cell to a wide range of static and dynamic mechanical stress conditions. An individual cell can be exposed to a sequence of mechanical stresses such as OT extension or compression, hydrostatic pressure, or fluid induced shear or extension. These stress conditions can be applied sequentially or simultaneously. With the $\mu$ PIVOT 
instrument combined with microfluidics, an entire mechanical stress sequence can be applied without changing equipment, altering the culture media, or examining a completely new cell and can be implemented quickly with minimal cell deterioration due to culture time. Moreover, with the imaging capabilities of $\mu \mathrm{PIV}$, local velocity fields may be calculated and cell morphology determined. From the velocity field, the stresses applied to the cell at any location (within the focal plane) along the cell membrane/fluid interface may be computed. The following preliminary results show the capabilities of the instrumentation on biological cells.

\subsubsection{OPTICAL-INDUCED STRESS}

\subsubsection{Cell viability during optical tweezing}

To our knowledge, no viability tests have been performed specifically for bone and cartilage cells subjected to optical tweezers. Although not our primary focus, quantifying cell viability is necessary to assure cell health during experiments. Numerous studies have investigated cell health and viability under optical traps through assays such as cell proliferation, cell mobility, and DNA structure. These studies show that the cell viability depends on the trapping wavelength, the power density, energy density, and the exposure duration. Liang et al. [Liang, 1996] showed no adverse effect on hamster ovary cell cloning efficiency when trapped with a $1064 \mathrm{~nm}$ laser at $175 \mathrm{~mW}$ for less than three minutes. Under similar conditions, Neuman et al. [Neuman, 1999] 
examined bacteria mobility (Escherichia coli) and observed limited photodamage due to optical traps. Liu et al [Liu, 1995] investigated the effect of $1064 \mathrm{~nm}$ laser on DNA structure, cell viability and $\mathrm{pH}$ levels of hamster ovary cells as well as human sperm cells: no effect was observed when the laser power was under $300 \mathrm{~mW}$ for less than 2 minutes.

Two effects of laser trapping on biological specimens are laser-induced heating and photodamage. With water being the main component of biological cells (approximately 70\%), laser induced heating is relatively mild for laser wavelengths of 200-1100 nm, where water absorption is small. Liu et al. [Liu, 1995] showed that for a laser operating at $1064 \mathrm{~nm}$ laser-induced heating is $1.15^{\circ} \mathrm{C}$ for every $100 \mathrm{~mW}$ of laser power entering a hamster ovarian cell trapped in a stationary fluid. Thus, for our experiments where the laser power is $30 \mathrm{~mW}$ and a cell is trapped in a moving fluid (more efficient heat transfer), we would expect a cell temperature increase of less than $0.38^{\circ} \mathrm{C}$, if we assume the relationship between temperature increase and laser power follows Liu et al. [Liu, 1995] and is linear. This level of laser-induced heating is assumed to have minimal influence on cell health.

To assess any optical damage of trapped cells, cell morphology was monitored and compared to non-trapped cells. In general, healthy live cells have distinct edges, are smaller in volume, and appear smoother, denser and more contained than dead cells. To determine viability, Trypan Blue, known to enter the intracellular space due to 
an increase in membrane porosity of dead or dying cells [Sunk, 2006], was introduced at a 1:1 volume ratio with culture medium. A morphology and viability benchmark was determined by trapping cells at maximum power ( $1 \mathrm{~W}$ at sample). Figure 73 provides the evolution of Trypan Blue uptake and net cell volume increase under these conditions. The results show a slight (visually insignificant) net cell volume increase and Trypan Blue uptake (decrease in intensity) during the first twenty seconds of applied laser power followed by a rapid increase (visually apparent) in net cell volume and Trypan Blue uptake. This indicates a change in the permeability of the cell membrane, a sign of cell damage. Within 35 seconds, Trypan Blue was clearly visible in the cell (Figure 73).

During cell biomechanics experiments, we monitored cell morphology and Trypan Blue uptake (if added). At typical laser powers (30 $\mathrm{mW}$ measured at the sample) no morphological change was observed over the experimental timeframe, an average of 20 minutes. 


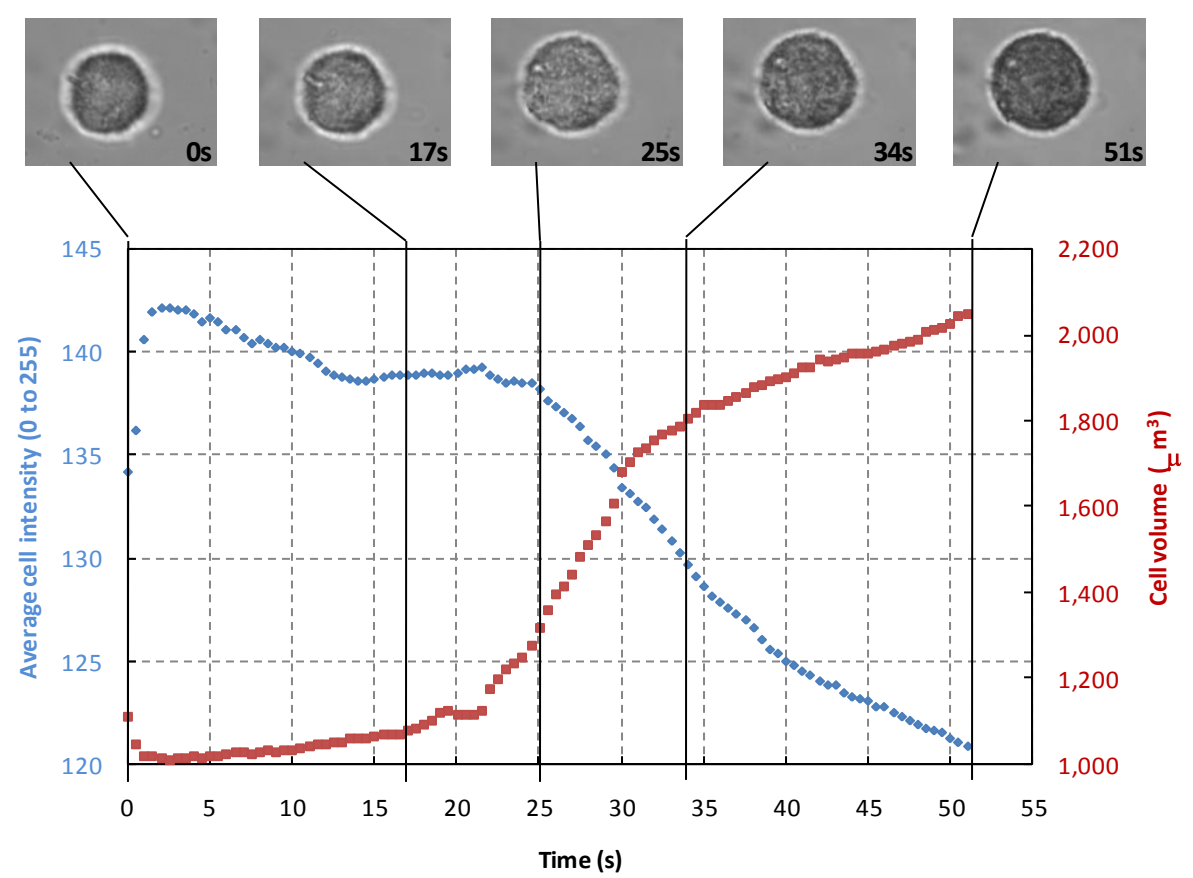

Figure 73: Representation of pixel intensity and volume changes of a $15.5 \mu \mathrm{m}$ diameter chondroblast under a high powered optical trap ( $\sim \mathrm{W}$ at sample), with representative pictures at determining time points. The cell membrane becomes permeable after $\sim 20$ seconds and the volume increases suddenly. After 35 seconds, the uptake of Trypan blue by the cell becomes visible.

\subsubsection{Cell manipulation by two optical traps}

The most common method to stretch cells or smaller entities such as macromolecules is to trap attached beads arranged as "handles" [Sleep, 1999],[Mills, 2004]. The primary advantage of this technique is the ability to induce higher cell deformation without inflicting increased optical damage to the cell (the laser energy is focused on the trapped beads). As the presence or absence of focal adhesions of a cell to a surface is known to alter the cytoskeleton, a potential disadvantage of this technique is the method of attachment of the bead to the cell surface. Since actin stress 
fibers are anchored at focal points and spread through the intracellular arrangement, cell-surface attachments modify a cell's shape and motility.

In order to gain insight on the elastic properties of the cell without the effects of physical attachment, we direct two optical traps directly into the cell and focus on intracellular organelles. Liao et al [Liao, 2008] used this method to stretch a trapped red blood cell by jumping the focal point of an optical tweezers between two locations at $100 \mathrm{~Hz}$. Figure 74 shows the physical response of a dead and live chondroblast subjected to opposed relative movement of the optical traps. The sequence of Figure 74(a), (b), (c) shows trapping of an organelle inside a dead cell. This organelle can be pulled out of the cell with limited resistance Figure 74(c). This organelle could be a number of cell sub-units approximately $1 \mu \mathrm{m}$ in size such as a small mitochondrion, a lysosome, a vacuole, or a vesicle. Wei et al. [Wei, 2008] have trapped submicron organelles of epithelial cells. In Figure 74(b), the cell deformation is at a maximum with a Taylor deformation parameter ( $D_{12}$ defined previously) of 0.15 . On the contrary, Figure $74(e)$ shows that the viable cell is slightly stretched at its maximum deformation of 0.05 . Any attempt to induce further deformation by additional movement of the OT resulted in the cell disconnecting from the dual traps and repositioning around a single trap. This suggests that one can probe the viability of a cell by monitoring the deformation characteristics of the cell and/or the rearrangement of the cell's internal structure. 
"Dead" chondroblast
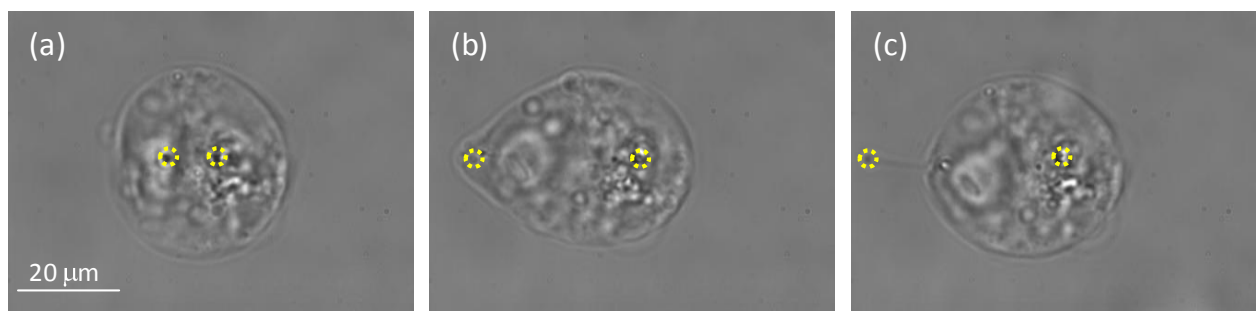

Live chondroblast
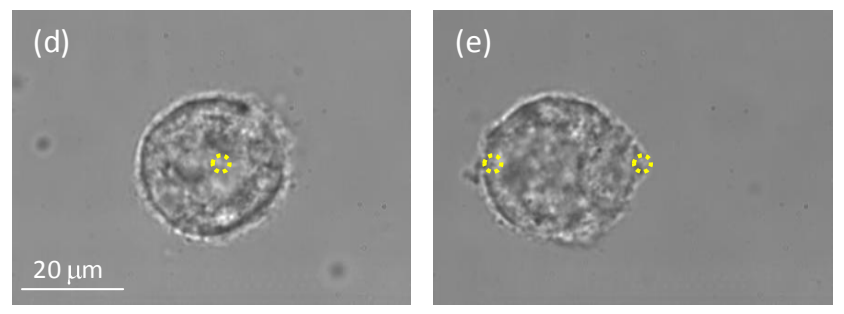

Figure 74: The deformation of chondroblasts by the relative movement of 2 optical traps. The dashed circles show the trap locations.

It is interesting to note that while an alive or dead cell stretches throughout its volume, a local deformation (a small protrusion) around the vicinity of the laser focus is observed (Figure 74(e)).

Recent AFM studies have shown that the stiffness of bacteria can either increase or decrease after death. Francius et al. [Francius, 2008] showed a decrease in cell stiffness after the digestion of the cell wall by Lysostaphin. On the contrary, Cerf et al. [Cerf, 2009] showed an increase in cell stiffness after deadly heating of the entire cell, which is hypothesized to collapse the lipopolysaccharides of the outer membrane layer and folding of some lipoproteins. Coupled with this research, these studies suggest 
that the stiffness of a dead cell depends not only on the cell type but on the method of cell death.

\subsubsection{HYDROSTATIC PRESSURE-INDUCED STRESS}

Hydrostatic pressure is a classic physical stress, known to induce and maintain complex reactions in living cells. In vivo variations in hydrostatic pressure induced from body weight and normal activity constantly acts on bone and cartilage cells. These pressure variations are known to play an important role in mechanotransduction. In vitro studies performed on osteoblast cultures have shown that mechanical stimulation by hydrostatic compression plays a role in regulating osteoblast metabolism, promoting the synthesis of signaling molecules and other molecules pertinent to new bone formation [Roelofsen, 1995], [Glantschnig, 1996], [Ferraro, 2004]. Focusing on only the mechanical response, Wilkes and Athanasiou [Wilkes, 1996] have demonstrated that osteoblast-like cells, suspended in media, are incompressible under hydrostatic pressures to up to $7 \mathrm{MPa}$. Following conventional hydrostatic compression procedures, Smith et al.[Smith, 1996] and Parkkinen et al.[Parkkinen, 1993] observed cellular and metabolic responses to increases in hydrostatic pressures up to $10 \mathrm{MPa}$ including a significant increase in the ${ }^{35} \mathrm{SO}_{4}$ uptake for a $0.5 \mathrm{MPa}$ load during $50 \mathrm{~ms}$ repeated at 4-s intervals. Toyoda et al.( (Toyoda, et al., 2003) applied hydrostatic pressure at 5 MPa for a four-hour loading period to chondrocytes cultured in 3-dimensional agarose gels. They observed a change in proteoglycan metabolism but no cell deformation. However a 
cellular response was detected for pressures as low as $5.86 \mathrm{kPa}$ where continuous hydrostatic pressure enhanced the calcium intake and inhibited the accumulation of cAMP in cartilage cells [Bourret, 1976].

In this study chondrocytes were trapped and hydrostatic pressure, $P_{\text {static }}$, varied. $P_{\text {static }}=\rho g h$, where $\rho$ is the fluid density $\left(\rho=10^{3} \mathrm{~kg} \mathrm{~m}^{-3}\right), g$ is gravity $\left(g=9.81 \mathrm{~m} \mathrm{~s}^{-2}\right)$, and $h$ is the height difference $(0$ to $20 \mathrm{~cm}$ ) between the input and output syringes. The laser power was set as low as possible, but sufficient to suspend a cell and position it in the microscope focal plane. For the limited range of hydrostatic pressures examined ( 0 to 2 $\mathrm{kPa})$, no significant volume change was recorded due to a change in hydrostatic pressure. This is not surprising as the pressures applied are quite small when compared to other studies that show no deformation at significantly higher pressures. With the applied technique, the maximum pressure that can be applied to an optically suspended cell is limited by the structural integrity of the coverslip. The microfluidic interconnects, chip materials (other than the coverslip), and chip bonding methods can withstand pressure in excess of 1.5MPa. A previous study examining coverslip strength reports coverslip failure at pressures of $\sim 200 \mathrm{kPa}$ [Peake, 2000]. At this maximum pressure $(200 \mathrm{kPa})$ it is highly unlikely that a significant volume change would occur for an optically suspended cell. However, it is still unclear whether small pressure perturbations up to 200kPa can induce a biological response. Smith et al. [Smith, 1996] and Parkkinen et al. [Parkkinen, 1993] observed a biological response at 500kPa. Further 
experiments at higher pressures that monitor the biological response are necessary to explore this possibility.

\subsubsection{HYDRODYNAMIC-INDUCED STRESS: UNIFORM FLOW}

A number of cell monolayer studies show that fluid flow is an influential mediator in bone remodeling and that the signaling response of osteoblasts depends on the flow profile. Reich et al.[Reich, 1990], Johnson et al.[Johnson, 1996], Chen et al.[Chen, 2000] and Kapur et al.[Kapur, 2003] have shown that osteoblasts respond to laminar flow shear stresses by changing their concentration of biochemical signals such as Nitric Oxide and intracellular $\left[\mathrm{Ca}^{2+}\right]$. McAllister et al. [McAllister, 1999] discovered that flow transients had greater effect on the stimulation of Nitric Oxide production. You et al.[You, 2001], Donahue et al [Donahue, 2003] and Mullender et al.[Mullender, 2006] observed that the biochemical response of osteoblasts increased during oscillating flows. This effect depends on both the flow amplitude (shear stress from 0.6 to $4 \mathrm{~Pa}$ ) and frequency. Kwon et al.[Kwon, 2007] examined the morphological response of adhered osteoblasts to steady and oscillating flows. Their results suggest that viscous deformation occurs during steady flow, while elastic deformation develops during oscillatory flows of physiological frequency $(\sim 1 \mathrm{~Hz})$.

The above studies demonstrate the importance of fluid flow on mechanotransduction. However, in these cell monolayers studies, cells are attached to a glass slide (and frequently attached to each other). Therefore, the cells are 
mechanically constrained and stimulated unevenly throughout their body. With the $\mu$ PIVOT, a single OT can hold a cell against an imposed fluid flow generated by the movement of the automated stage. Under these conditions, a cell is subjected to three dimensional stresses with no physical attachment. Two experimental parameters are examined here in response to this flow state, the cell deformation, and the trap stiffness.

\subsubsection{Cell deformation}

Figure 75 shows (a) a chondroblast in a quiescent fluid, and (b) the same chondroblast subjected to a unidirectional flow of $50 \mu \mathrm{m} / \mathrm{s}$ fluid velocity. Figure 75 (b) shows a clear shift of the cell to the right during flow conditions with the cell flattened slightly on the upstream face due to hydrodynamic pressure. The calculated cell deformation was $D_{12}=0.03$.

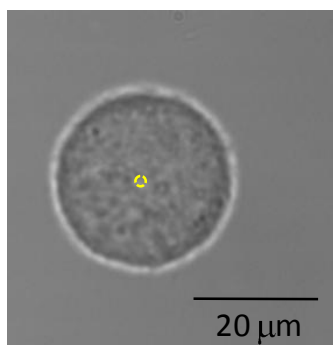

No flow

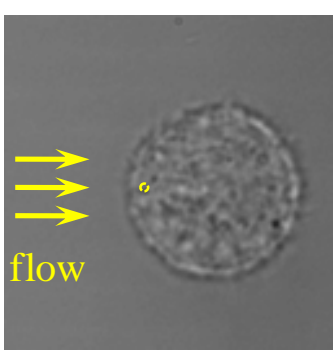

$50 \mu \mathrm{m} / \mathrm{s}$ straight flow

Figure 75: A $19.1 \mu \mathrm{m}$ diameter chondroblast in static suspension (left). Visible cellular deformation of the cell due to an applied fluid shear stress induced by a straight channel flow (right). The circle represents the trap size (approximately $1.6 \mu \mathrm{m}$ in diameter) and location. 
Figure 76 shows the average cell deformation measured at each flow velocity for all cells tested. As expected, the cell deformation increases with the applied force. The two data points at zero velocity corresponds to the cell before and after the velocity sequence was applied. It is interesting to note that the cell regains its non-deformed shape when the drag forces are eliminated.

For the applied laser power ( $30 \mathrm{~mW}$ at the sample to avoid cell photodamage), the drag force can easily exceed the optical trap force. Therefore, the magnitude of induced fluid stresses is limited and larger cell deformation is not possible. For these experiments, the maximum applied flow velocity was $100 \mu \mathrm{m} / \mathrm{s}$ which, with a culture media viscosity of $\mu=1 \mathrm{mPa}$.s, a cell radius, $a=10 \mu \mathrm{m}$, and the distance to the coverslip, I $=30 \mu \mathrm{m}$, corresponds to a maximum drag force of approximately $28 \mathrm{pN}$.

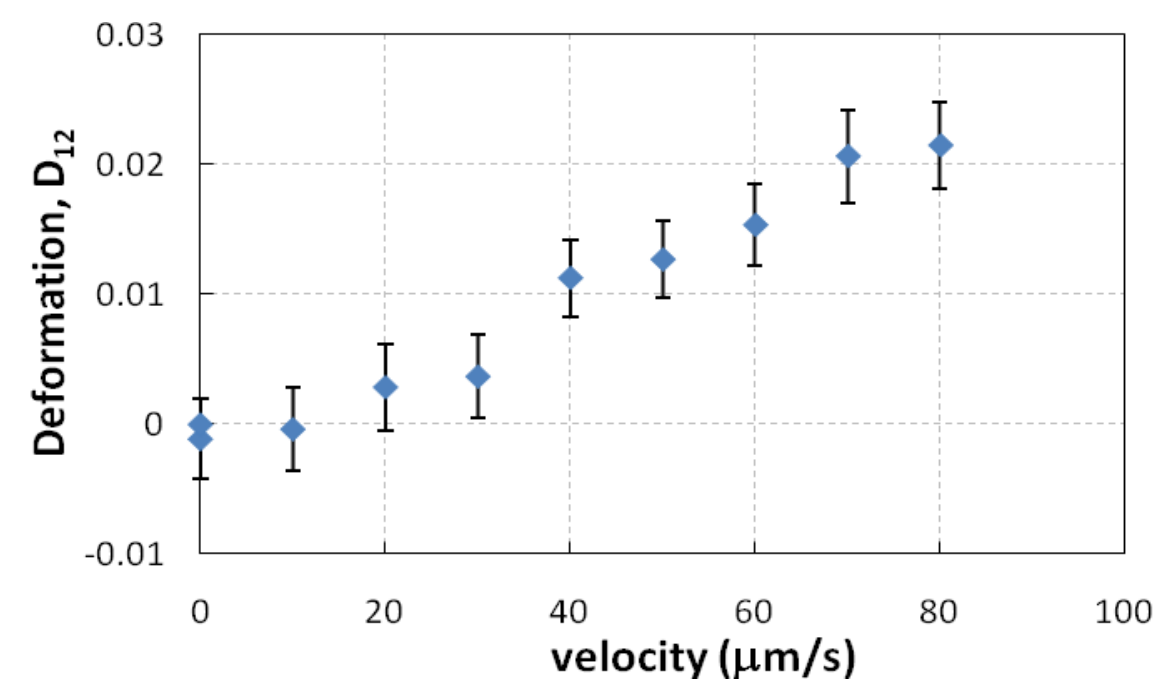

Figure 76: Average deformation as a function of flow velocity for 64 cells tested. The two data points at 0 velocity are taken before and after the experiment.

157 
Assuming the cell is a solid rigid sphere subjected to uniform creeping flow, the maximum shear $(\tau)$ and normal $(\sigma)$ stresses are (see Chapter 8$) \tau_{\max }=\sigma_{\max }=3 \mu v_{\infty} / 2 a$, where $\mu$ is the fluid viscosity, $a$ is the cell radius, and $v_{\infty}$ is the fluid velocity. With the culture media viscosity equal to $1 \mathrm{mPa} . \mathrm{s}$ and a cell radius of $10 \mu \mathrm{m}$, the maximum shear stress applied to the cell was approximately $15 \mathrm{mPa}$. While this fluid induced stress is roughly 60 times smaller than typical shear stresses applied uniformly along only the exposed cell surface during cell monolayer studies, the stress is varied across the entire cell surface. In this experiment the cell is indeed entirely exposed to the flow instead of having a large attached surface not experiencing any fluid stress.

\subsubsection{Trap stiffness}

As described earlier, trap stiffness is calculated by measuring the cell displacement from its equilibrium, no flow position. Trap stiffness is known to depend, among other parameters, on the properties of the object being trapped. Therefore, trap stiffness may be a source of information to characterize cellular properties. For example, cells could be the same type (for example chondroblasts) but have dissimilar actin filament distributions or orientations, intracellular fluid composition, etc., due to a difference in their location (e.g. different layers of cartilage) or healthy versus diseased states. The differences in their intracellular constitution could affect the trap stiffness, and thus provide a means to identify influences on cell behavior. In this study, fifty-one chondroblasts and osteoblasts were trapped in a straight flow and their corresponding 
trap stiffness calculated. The purpose of this study was to determine the reproducibility of the experiment, the potential range of linear trap stiffness values, and the plausible difference in $\mathrm{k}$ with cell variables.

We know from the polystyrene sphere experiments (previous chapter) that the trap stiffness is a function of the size of the trapped object with the relationship

$k=C / d$, with $k$, the trap stiffness, $C$ the constant depending on multiple variables, and $d$ the diameter of the trapped object. For our cell experiments, we wanted to exclude the size effect on the trap stiffness. A new parameter, $k_{d}$, was therefore calculated instead of the trap stiffness. It is size independent and was calculated as the slope from the curve of the drag force multiplied by the cell diameter, as a function of cell displacement:

$$
k_{d}=k \cdot d
$$

This removes the size dependence on trap stiffness and allows cells to be differentiated by their material properties. Figure 77 shows this modified trap stiffness is confined within the range of 7.1 and $15.4 \mathrm{pN}$ (average= $=11.1 \mathrm{pN}$, standard deviation=2.1 pN). This wide range yields a potential $54 \%$ difference in trap stiffness between cells. 


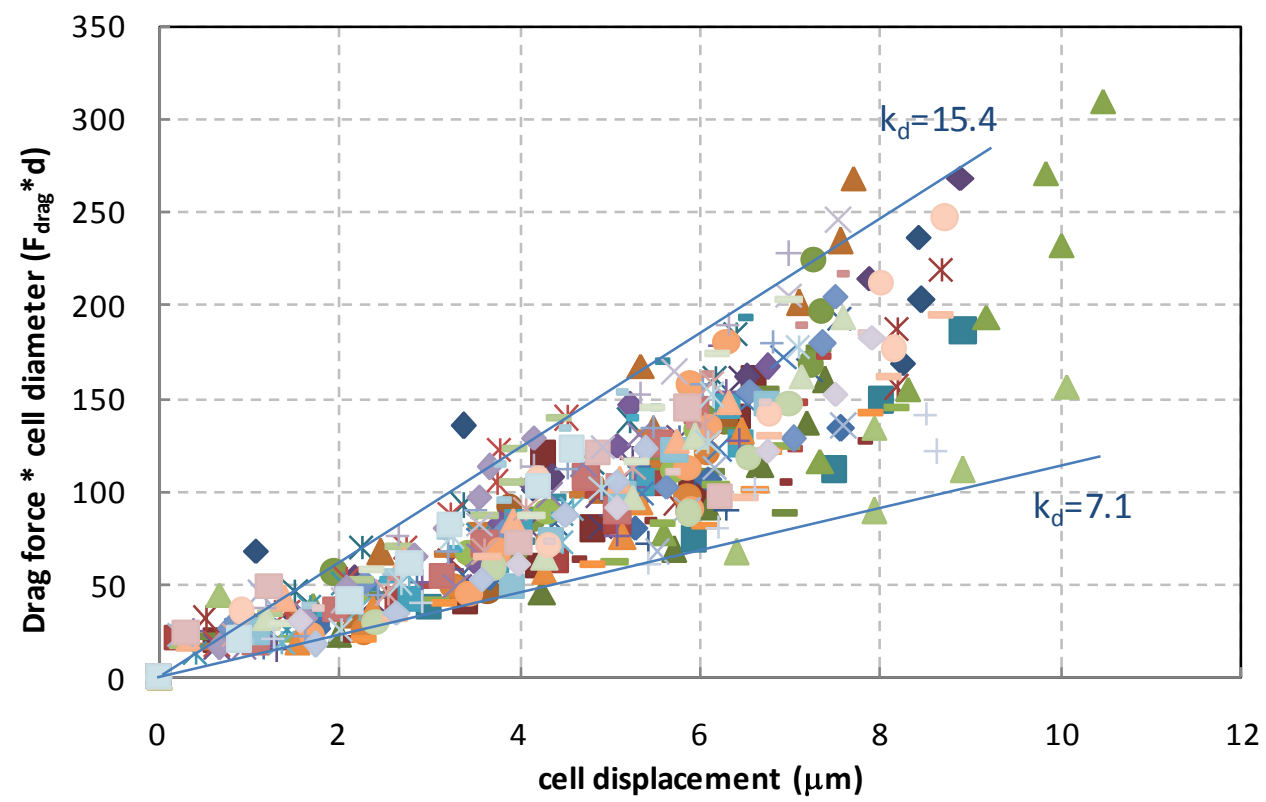

Figure 77: Cell displacement as a function of fluid drag for chondroblasts and osteoblasts with 11.4 to $23 \mu \mathrm{m}$ diameters. The modified trap stiffness $\left(k_{d}\right)$ is determined from the slope of Fdrag.d versus $\Delta x$. The maximum variation in modified trap stiffness is $54 \%$. The lines represent the maximum and minimum trap stiffness calculations.

Figure 78 shows a representative result for multiple trap stiffness measurements on the same cell. This $\mathrm{P} 2,16.5 \mu \mathrm{m}$ diameter chondroblast was initially tested under an OT power of $30 \mathrm{~mW}$ then $130 \mathrm{~mW}$ for 3 minutes under an applied drag force. The cell was then re-tested at $30 \mathrm{~mW}$. The total experiment time of was 15 minutes and no morphological change or reorientation of the cell was observed. The average trap stiffness was measured to be $1.2 \mathrm{pN} / \mu \mathrm{m}$ with a difference of $3.7 \%$ between the two runs. 


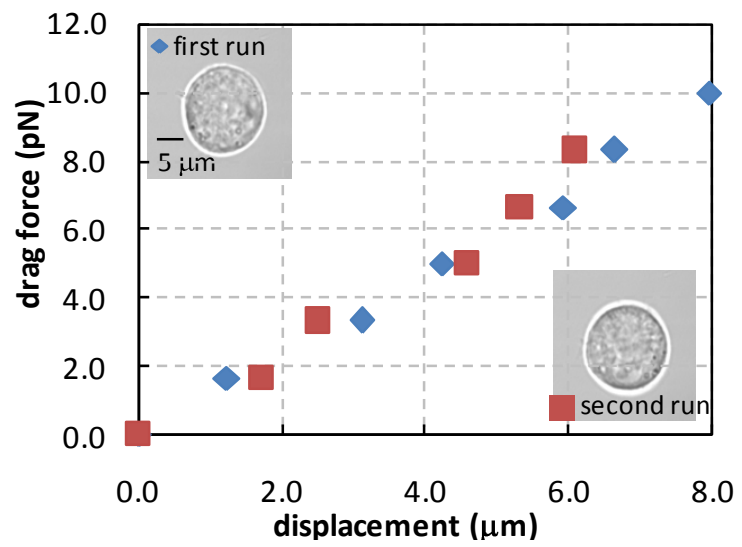

Figure 78: Drag force versus displacement for a $16.5 \mu \mathrm{m}$ diameter chondroblast (P2). The experiment was repeated with the same cell to assess the variability due to the measurement technique. The trap stiffness was measured to be approximately $1.2 \mathrm{pN} / \mu \mathrm{m}$ with a difference of $3.7 \%$ between the two runs.

The range of variations of trap stiffness for all the cells studied in two consecutive runs under the same conditions was calculated to be between $0.9 \%$ and $8.6 \%$ (average $=3.7 \%$, standard deviation=3.5\%), with a typical difference of 3 to $4 \%$ which is consistent with the $5 \%$ expected uncertainty in the drag force calculation. These results show a small trap stiffness variability and indicate that an individual cell may have a preferred trapped configuration. Thus, the errors in the measurement technique are insufficient to generate the wide range observed in Figure 77. This indicates that the variation in Figure 77 is not due to the measurement technique, but due to the variations in cell properties (age, culture time, passage, morphology, size, etc.).

In order to test this hypothesis, and potentially identify cell properties through trap stiffness measurements, single-factor ANOVA was conducted on 64 cells that differ in type (chondroblast or osteoblast). For the chondroblast population (52 cells) we 
studied the trap stiffness on cells with different passage (P1, P2 or P3), surface

roughness (values from 1 to 5 were assessed with 1 being very smooth and 5 being very granular), time between experiment and trypsinization, number of cilia, size of cilia, and cell concentration during transport from extraction laboratory (OHSU) to experiments laboratory (Microscale Laboratory, PSU).

No significant trap stiffness difference was observed between chondroblasts (average $=11.0 \pm 2.1 \mathrm{pN}$ ) and osteoblasts (average $=11.4 \pm 1.9 \mathrm{pN}$ ). Additionally, focusing on the chondroblast population, the surface roughness, the size of cilia and the cell concentration had no statistical effect on the trap stiffness (Table 3).

\begin{tabular}{|c|c|c|}
\hline Groups & Variables & $\mathbf{k}_{\mathrm{d}}$ (in $\mathbf{p N}$ ) \\
\hline \multirow{4}{*}{ Surface roughness } & very smooth & $10.4 \pm 1.8$ \\
\cline { 2 - 3 } & smooth & $10.9 \pm 2.1$ \\
\cline { 2 - 3 } & irregular & $10.3 \pm 1.4$ \\
\cline { 2 - 3 } & granular & $10.9 \pm 2.0$ \\
\cline { 2 - 3 } & very granular & $12.3 \pm 2.5$ \\
\hline \multirow{5}{*}{ Cilia size } & very small & $11.6 \pm 3.6$ \\
\cline { 2 - 3 } & small & $10.8 \pm 1.9$ \\
\cline { 2 - 3 } & medium & $9.2 \pm 1.1$ \\
\cline { 2 - 3 } & long & $9.9 \pm 1.1$ \\
\cline { 2 - 3 } & very long & $9.5 \pm 1.3$ \\
\hline \multirow{5}{*}{ Concentration } & $10.10^{3}$ cells $/ \mathrm{mL}$ & $9.9 \pm 3.6$ \\
\cline { 2 - 3 } & $20.10^{3}$ cells $/ \mathrm{mL}$ & $11.1 \pm 2.2$ \\
\cline { 2 - 3 } & $30.10^{3}$ cells $/ \mathrm{mL}$ & $11.2 \pm 2.1$ \\
\cline { 2 - 3 } & $40.10^{3}$ cells $/ \mathrm{mL}$ & $9.9 \pm 0.7$ \\
\cline { 2 - 3 } & $50.10^{3}$ cells $/ \mathrm{mL}$ & $10.5 \pm 1.6$ \\
\hline \multirow{7}{*}{$100.10^{3}$ cells $/ \mathrm{mL}$} & $11.6 \pm 3.6$ \\
\hline
\end{tabular}

Table 3: Average modified trap stiffness for chondroblasts split into different groups. No significant difference due to surface roughness, cilia size or cell concentration. 
Figure 79 shows a slight decrease in $k_{d}$ with an increase in passage as a result of chondroblasts de-differentiating. However, this trend is statistically insignificant as the sample size is insufficient. (Note: In the following graphs, the error bar represents the standard error, which is calculated by dividing the standard deviation by the square root of number of samples in the group. This allows to account for the sample size, which would not be the case if only using the standard deviation as the error bar. The values above the columns indicate the average modified trap stiffness for each group.)

While the above parameters showed no statistical variation, two variables were statistically significant. The first one is the number of cilia on the cell surface. The second one is the time spent between the extraction of the cells from the culture flask (trypsinization), and the experiment.

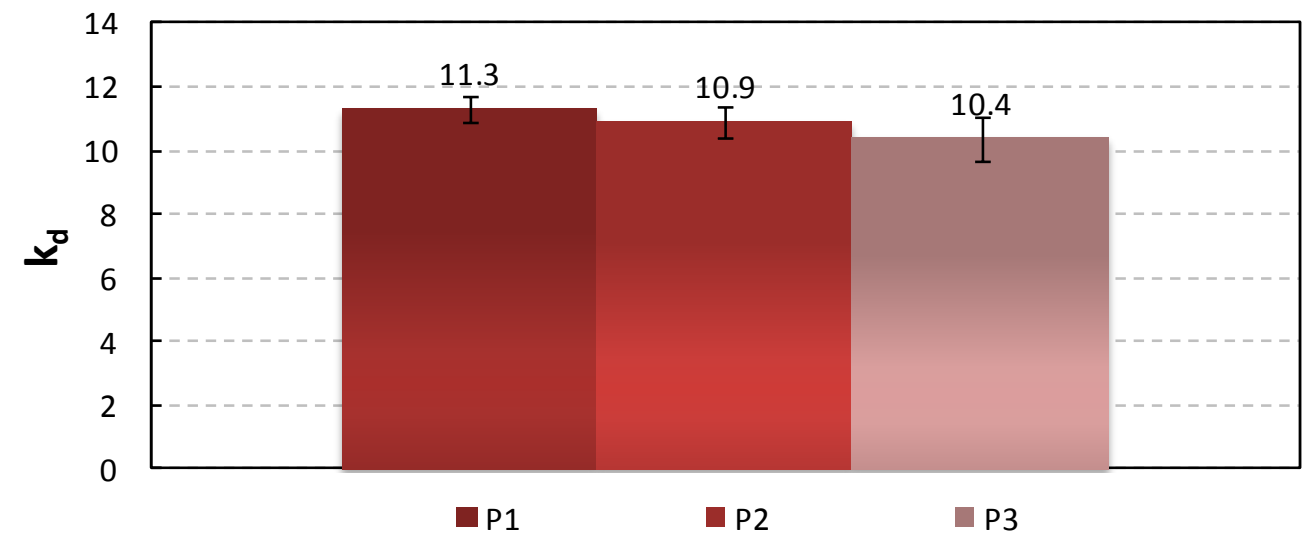

Figure 79: Modified trap stiffness for chondroblasts with different passages (P1, P2, P3). No significant difference was observed, but we can see a trend of trap stiffness decreasing with increasing dedifferentiation. 
For the number of cilia, we observed a significant difference between the cells that did not possess any cilia compared to those that had more than one cilium on their surface ( $P$-value $=0.047)$. Figure 80 shows that the fewer cilia the cell has, the stiffer the trap is. This could be explained by the fact that the presence of the cilia changes the optical properties of the cells. Some cilia, called primary cilia, are known to be a continuation of the cytoskeleton [Malone, 2007] Therefore not only the surface but the entire cell could be affected by the presence of these protrusions and hence have a detectable effect using our set up.

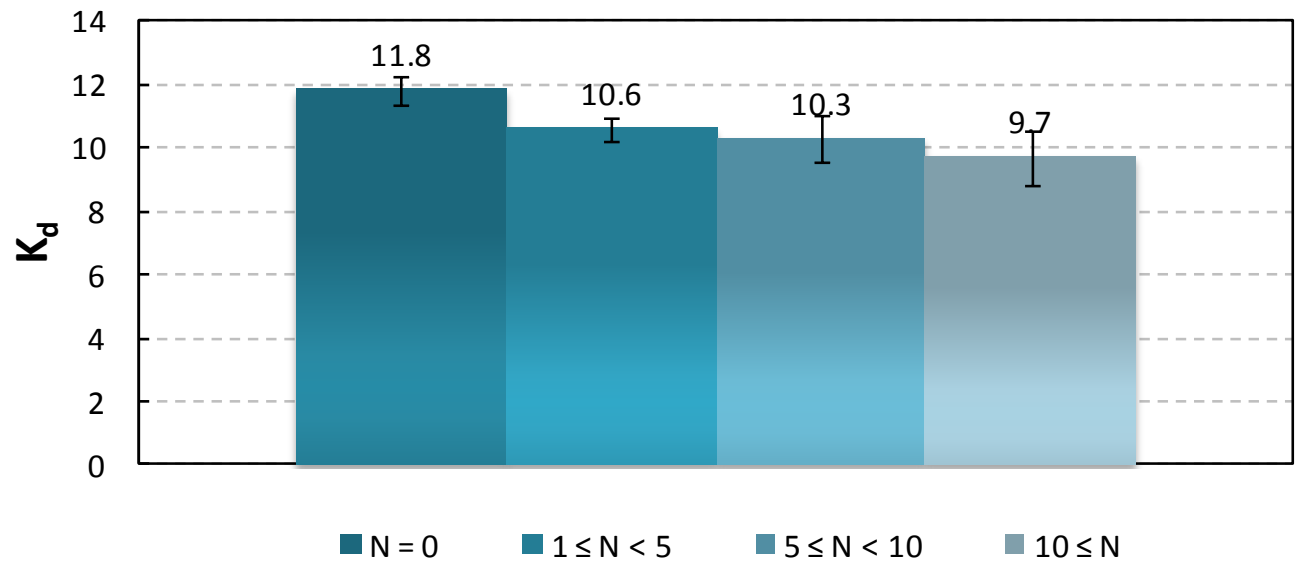

Figure 80: Modified trap stiffness for chondroblasts with different numbers of cilia (N) on their surface. As $\mathbf{N}$ increases, the trap becomes weaker. 
Finally, a significant difference $(P$-value $=0.0022)$ was also observed when examining the modified trap stiffness for cells tested the same day they were extracted from the culture flask and those who were tested the following day. Figure 81 shows $k_{d}$ increases with time. However, this difference may not be a result of just the elapse time between extraction and experiment. For the "next day" group, cells were placed in a $4{ }^{\circ} \mathrm{C}$ refrigerator and brought out at least two hours prior the experiment for a slow and gentle temperature raise to room temperature. Thus, it is difficult to pinpoint the exact reason why the trap is more efficient for cells processed the following day. However, it is safe to say that the way the cell is being processed between the time it is trypsinized and the time it is tested has an effect on its material properties.

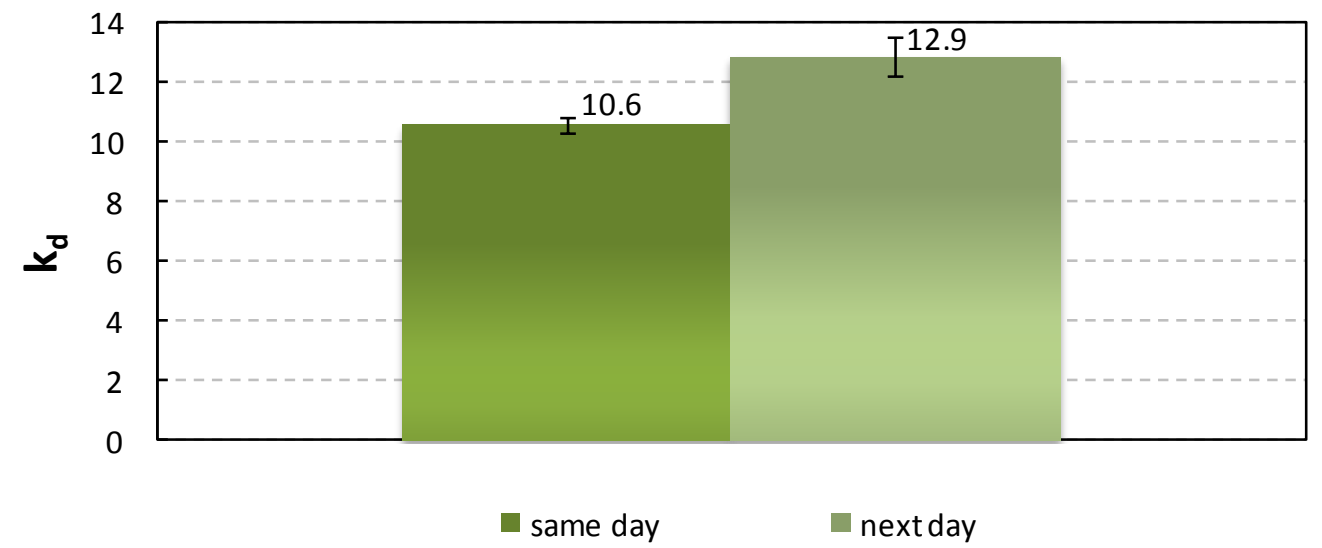

Figure 81: Modified trap stiffness for chondroblasts tested the same day they were extracted from culture flask, and the following day. 


\subsubsection{EFFECTS OF CELL ELONGATION AND CILIA ON DRAG FORCE: COMPUTATIONAL MODELING}

The above measurements show a statistical difference in the trap stiffness as a function of cilia. To determine whether this difference corresponds to an increase in fluid drag associated with cell elongation or protrusions, cells with different shapes in uniform flow were examined computationally and compared with the theoretical drag of a rigid sphere. As discussed earlier, two geometric effects were examined: the elongation of the cell up to the maximum elongation of $22 \%$ (see Figure 76 ) and the number and size of protrusions extending from the cell.

\subsubsection{Modeling of oval shapes in uniform flow}

Using the uniform flow velocity $(100 \mu \mathrm{m} / \mathrm{s}$ ) and boundaries (cell at $50 \mu \mathrm{m}$ from bottom wall) as in a typical experiment, cells with different elongations were tested. The fluid is flowing in the x-direction. For each model, the cell volume is unchanged and taken as the volume of a perfectly spherical $20 \mu \mathrm{m}$ diameter cell. For this configuration, the drag force was calculated to be $10.78 \mathrm{pN}$. In our experiments, a few cells showed an elongation in the $y$ or $z$ directions with a maximum of $10 \%$ elongation. The resulting drag force when modeled was $10.88 \mathrm{pN}$ for the elongation in the $\mathrm{y}$-direction, and 10.85 $\mathrm{pN}$ in the z-direction. Both of the results show less than $1 \%$ difference in the drag force calculation due to the deformation of the cell. In the $\mathrm{x}$-direction, models were tested with 10 to $50 \%$ elongation with $10 \%$ elongation increments. Figure 82 provides the 
results of the simulations. There is a steady decrease of the drag force with cell elongation. For the maximum elongation observed experimentally (22\%), the drag force was calculated to be $\sim 10.42 \mathrm{pN}$, which corresponds to a difference of $3.3 \%$ compared to the drag force for a spherical cell of the same volume. It should be noted, that this $22 \%$ elongation was recorded for the highest flow velocities. At this flow, the forces are no longer in the linear trap regime. For the linear trap stiffness measurements reported, the drag force was substantially lower and the resulting elongation quite small. Thus, the error in the drag force is less than $3.3 \%$.

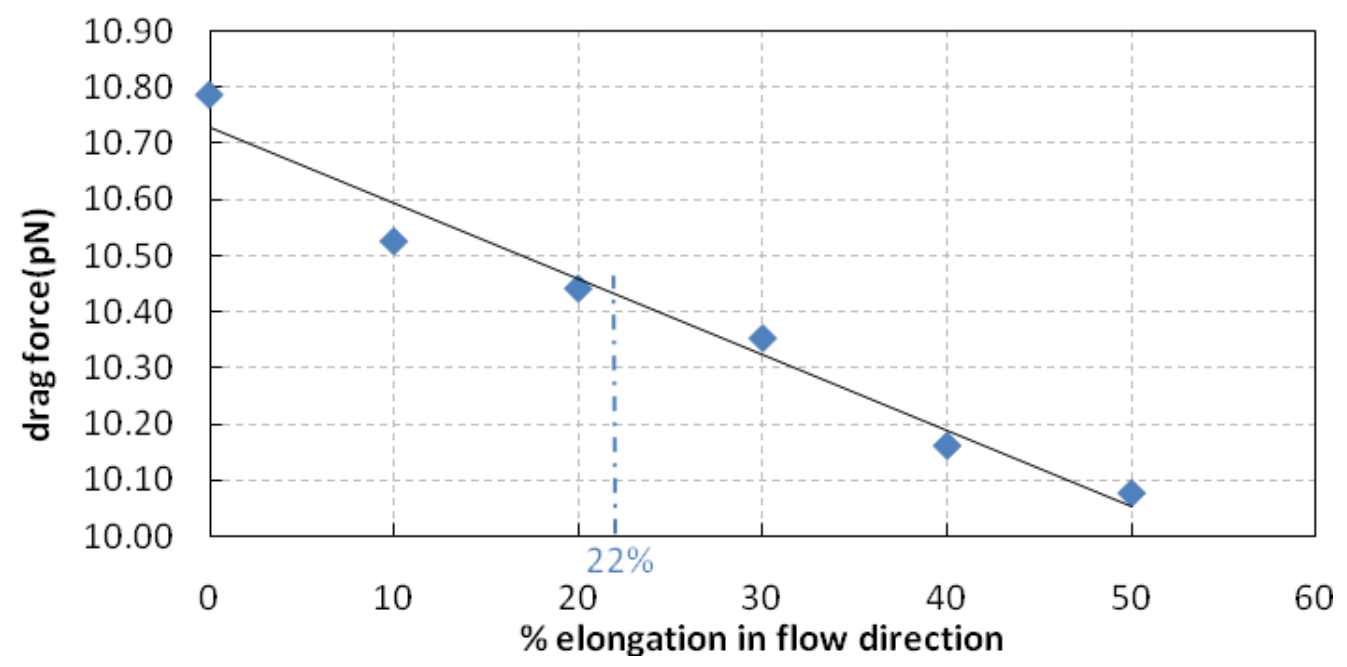

Figure 82: Drag force as a function of sphere elongation in the flow direction. $22 \%$ is the maximum deformation seen in our cell experiments. 


\subsubsection{Modeling of a spherical cell with protrusions in uniform flow}

It is important to note that the majority of the cells tested in uniform flows (46\%) did not have any visible protrusion therefore the modeling of uniform flow around a spherical cell was for most cases accurate (Figure 83), with a theoretical drag force equal to $10.78 \mathrm{pN}$.

However a few cells exhibit cilia or protrusion at their surface. As shown in Figure $84 a$ ), most protrusions are small and limited in number. This scenario represents approximately $27 \%$ of the cells tested. But as shown in Figure 84 (b), protrusions can be numerous ( $29 \%$ of the cells tested) and occasionally up to 1.5 times the cell radius (Figure 84(c)).

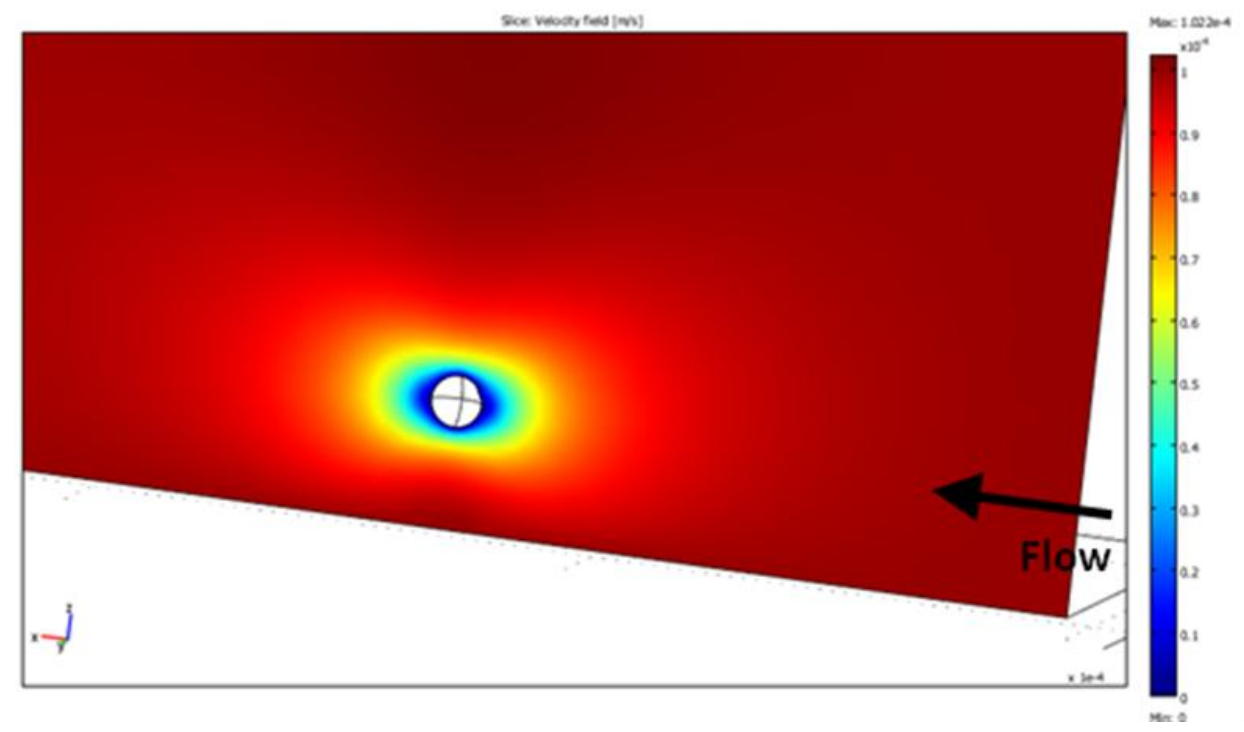

Figure 83: Modeling of a $10 \mu \mathrm{m}$ radius sphere in a uniform flow (x-direction), placed $50 \mu \mathrm{m}$ from the bottom coverslip. The colors represent the flow velocity in the $x-z$ plane with the scale on the right showing the flow velocity values from 0 (bottom/blue) to $10^{-4} \mu \mathrm{m} / \mathrm{s}$ (top/dark red). 

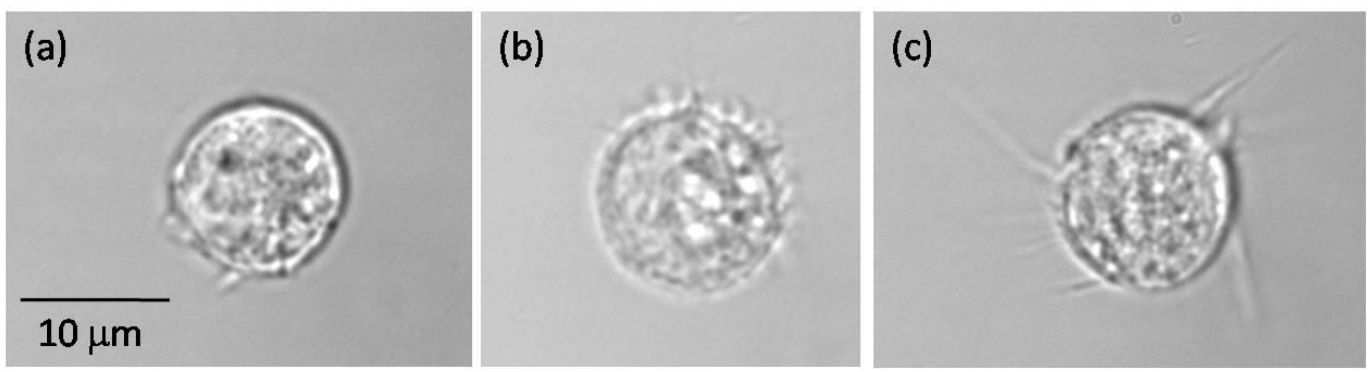

Figure 84: Pictures of chondroblasts with the 60x objective. (a) The cell has one small protrusion. (b) Multitude of small protrusions. (c) Very long protrusions affecting the drag force considerably.

To examine the effects of cell protrusions on the drag force measurement, a $10 \mu \mathrm{m}$ radius sphere was tested with variable protrusions on its surface. The presence of a protrusion in the flow direction has almost no effect on the drag force. A protrusion of 1.5 times the radius in the $\mathrm{x}$ or $-\mathrm{x}$ direction in fact decreases the drag force by $0.1 \%$. However, in any other direction, these long protrusions have a much higher impact and can affect the drag force considerably. Figure 85 (a) and (b) show the flow velocity around a cell possessing one protrusion in the $y$ - or z-direction respectively.

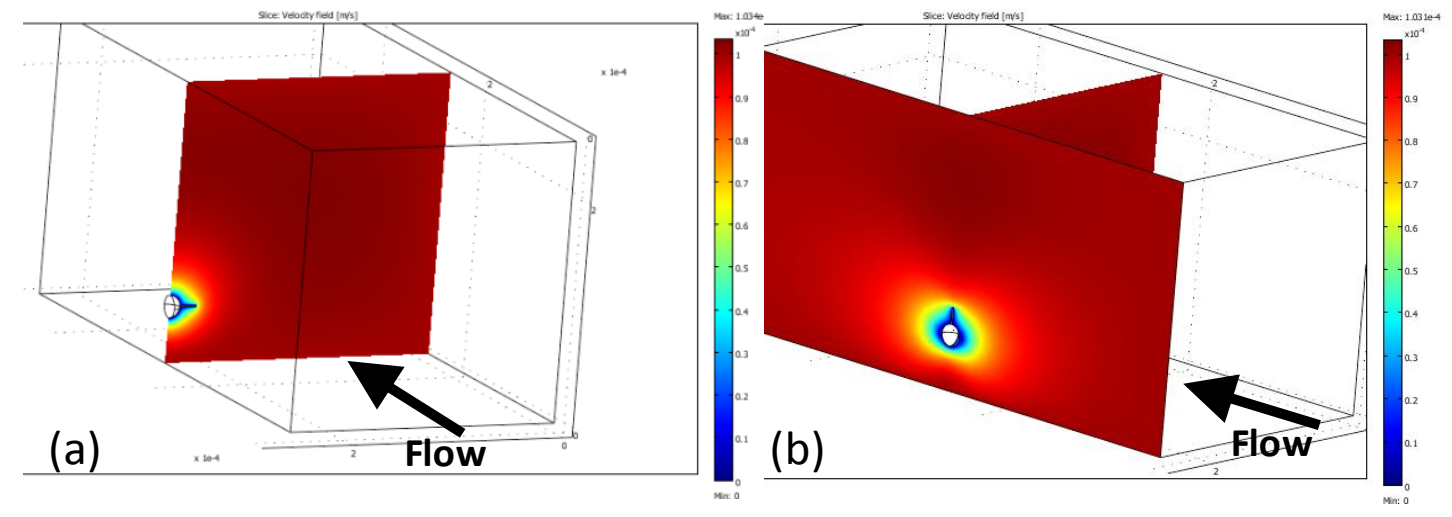

Figure 85: Model of flow velocity around a cell with a single protrusion in (a) the y-direction and (b) the z-direction. 
Its presence increases the drag force by $6 \%$. When there are four long protrusions (one in each of the main axes, and an extra one in the $y$-direction because of symmetry), the drag force increases $18.5 \%$. When there are six (Figure 86), the drag force increases $25 \%$. Overall, long protrusions are infrequent (less than $10 \%$ of the cells tested) and small protrusions have little effect on the drag force. The model of a cell with 26 protrusions measuring a fifth of the cell radius in length, increases the drag $0.2 \%$ compared to a cell the same size with no protrusion.

Thus, for most of the cells tested, the hypothesis that the cell is spherical for the calculation of the drag force is a good approximation. This assumption however breaks down for cells with long protrusions across the flow.

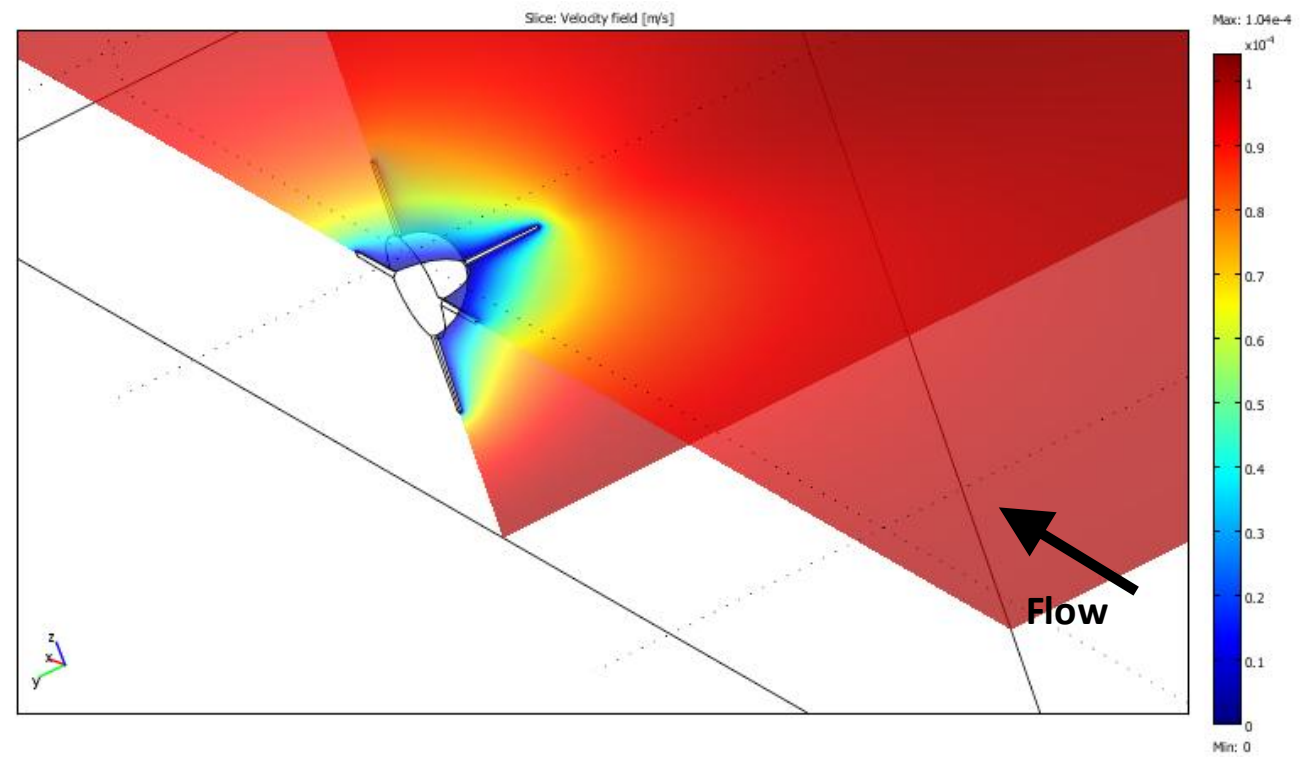

Figure 86: Model of flow velocity around a cell with a six protrusions in all the main axes. 


\subsubsection{HYDRODYNAMIC-INDUCED STRESS: EXTENSIONAL FLOW}

For uniform flows, the magnitude of fluid induced stresses is limited by the maximum optical trap forces that may be applied without optically damaging the cell. To apply stresses similar to cell monolayer studies, a laser power of $\sim 1 \mathrm{~W}$ would be required. As described earlier in this chapter, this would inflict cell damage within $\sim 20$ seconds and cell death after 35 seconds. Therefore, in order to apply similar fluid induced shear stresses on the cell without inflicting optical damage, flows in which fluid drag is negligible are required. As described earlier, a cross-junction flow geometry creates an extensional flow where the cell is compressed and stretched at the stagnation point. Theoretically, a cell centered at a stagnation point experiences no net drag force and remains there indefinitely regardless of the magnitude of shear/extensional rate. In practice, the stagnation point represents a saddle point, unstable to perturbations in cell position. However, the cell may be maintained at that location by applying small restoring forces (with the OT) to counteract any perturbations. These restoring forces are substantially smaller than the drag force on a cell in a uniform flow with equivalent shear rates. Maintaining the cell at the stagnation point eliminates the drag force thus minimizes the laser power required to apply higher fluid induced stresses. This reduces the possibility of deleterious heating and photodamage. Additionally, with the drag force equal to zero at the stagnation point, the maximum possible shear stresses may exceed those possible during cell monolayer 
studies and are only limited by the geometry and flow rate of the microfluidic crossjunction chips as well as the ability to position and maintain the cell at the stagnation point.

Figure 87 shows a live osteoblastic cell ( $20 \mu \mathrm{m}$ in diameter) optically trapped in a microfluidic cross-junction flow. This image illustrates the capabilities of the $\mu$ PIVOT to trap a cell and maintain its position at the stagnation point. For the experimental conditions of Figure 87 (extension rate, $\gamma=10 \mathrm{~s}^{-1}$ ), no deformation of the osteoblastic cell was observed. With a culture media viscosity of $\mu \approx 1 \mathrm{mPa}$.s and a current maximum undisturbed flow extension rate of $\gamma=50 \mathrm{~s}^{-1}$ (current manual positioning of the cell at the stagnation point limits further increases) the maximum potential stress that may be applied to a cell in a cross junction is approximately $\tau_{\max }=5 \mu \gamma=250 \mathrm{mPa}$ (see Chapter 8 for equation derivation).

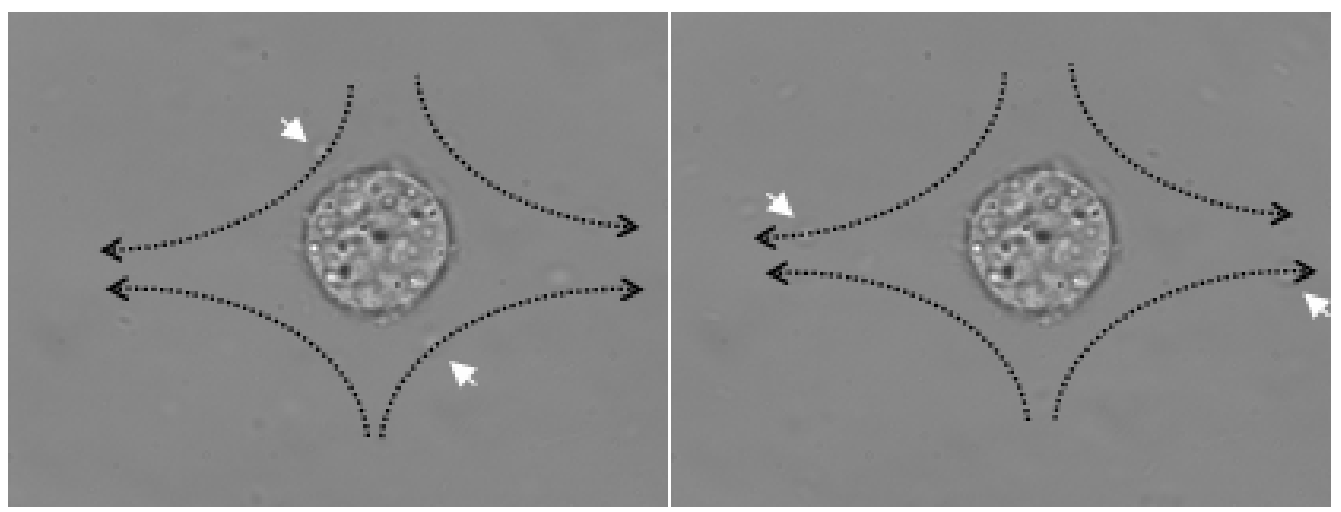

Figure 87: A living, $20 \mu \mathrm{m}$ diameter osteoblastic optically trapped in the microfluidic cross-junction flow. The small white arrows show flowing particles and the doted black arrows show the general flow direction. 
This is an order of magnitude higher than the maximum stresses achievable with uniform flow and a factor of $\sim 4$ smaller than those in cell monolayer studies.

Automation of the trap positioning and active control of the cell position relative to the stagnation point should enable substantially greater shear stresses (in excess of 1 Pa). Such control schemes have been used successfully to examine drop deformation in planar extensional flows [Bentley, 1986a], [Tretheway, 1999] over a large range of extension rates.

In contrast to the relatively stiff osteoblastic cell, Figure 88 provides the deformation of a myoblast (C2C12 muscle cells) as a function of the fluid extension rate in the cross junction.

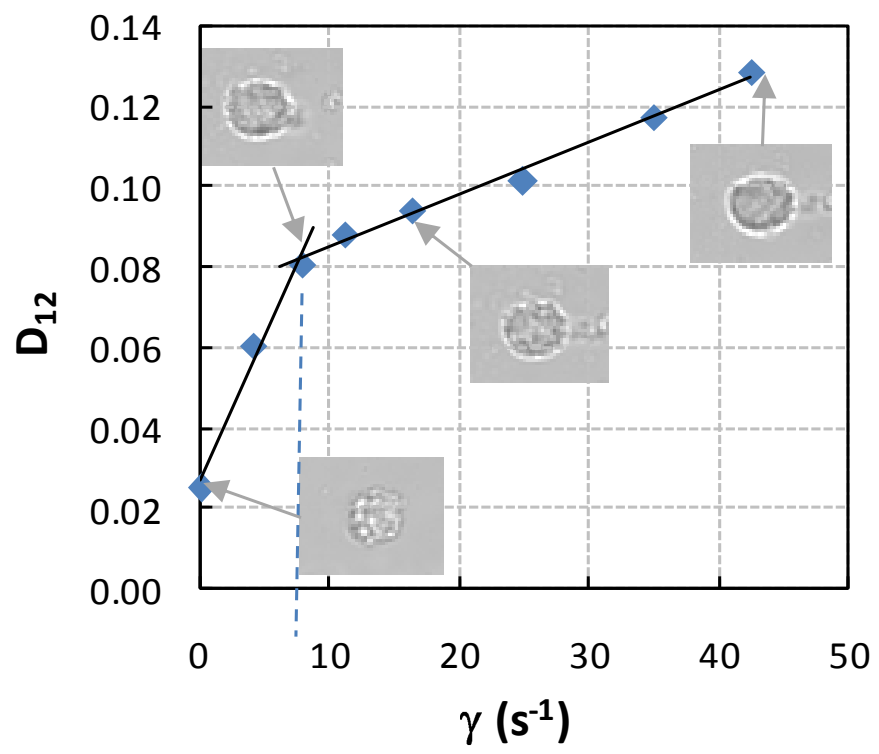

Figure 88: Deformation characteristics of a myoblast subjected to the cross junction extensional flow. Low extension rate $\left(<7.8 \mathrm{~s}^{-1}\right)$ and high extension rate $\left(>7.8 \mathrm{~s}^{-1}\right)$ deformation regimes are observed. 
Note that an initial asymmetry of the myoblast exists, $D_{12}=0.027$ at $\gamma=0$. At smaller extension rates, the cell deforms linearly with a slope of $\sim 6.9 \times 10^{-3} \mathrm{~s}$. At higher extension rates, the deformation is linear as well, but with a slope of $1.5 \times 10^{-3} \mathrm{~s}$. The two linear regimes were identified by linearly fitting the data starting from either the lowest or highest data point respectively and calculating the $\mathrm{R}^{2}$ value as each successive data point was added. The lines plotted represent a linear fit with $R^{2}>0.98$ that incorporated a maximum number of data points ( 3 points for the low regime and 6 points for the high regime). The results suggest a modulated response of the cell to the applied shear and normal stresses with a low extension rate regime below $\sim 7.8 \mathrm{~s}^{-1}$ and a high extension rate regime above $\sim 7.8 \mathrm{~s}^{-1}$. From a drop deformation perspective, this behavior would be consistent with a non-Newtonian shear thickening material.

To the best of our knowledge, the above experiments represent the first time an osteoblast or myoblast has been optically suspended and manipulated in a extensional flow microenvironment. (Hudson et al. [Hudson, 2004] positioned a red blood cell in a low shear rate extensional flow environment by controlling the flow rates in opposite flow channels). This microfluidic manipulation and subsequent analysis may provide new insight on the response of cells to different mechanical stimuli. Moreover, the deformation results provided by these cell experiments could be directly compared to drop deformation analysis in extensional flows. Extensive drop deformation studies have been conducted with an emphasis on fluid type, Newtonian versus non-Newtonian 
[Bentley, 1986b], [Tretheway, 2001], visco-elastic bodies [Eggleton, 1999], and variations in interfacial properties [Tretheway, 1999]. Additionally, numerical and theoretical models incorporating these effects are relatively abundant [Ramaswamy, 1999]. With this difference in perspective, a drop deformation comparison may provide insight into modeling cell mechanics and help characterize the viscoelastic properties of cells.

\subsection{COMMENT ON RELEVANCE TO MECHANOTRANSDUCTION}

While single cell suspension is dissimilar from in vivo conditions, where bone and cartilage cells are living in a dynamic fluid and surface microenvironment, this technique may provide insight into the mechanotransduction process. Specifically, the mechanism associated with cell attachment is highly complex and not fully understood. By suspending a cell then incorporating controlled stresses and interactions including cell attachment, the effects of a specific interaction may be elucidated from other contributing factors. Additionally, with the imaging and velocity field characterization of $\mu \mathrm{PIV}$, the actual morphology and stress state of a suspended cell can be accurately measured. Thus, the cell's mechanical response can be directly related to the applied stresses without model interpretation of results. To further address

mechanotransduction, the cell biological response to mechanical stimulation must be characterized. Intracellular calcium concentration, as well as Nitric Oxide production are recognized to increase under certain types of mechanical loadings, and the actin 
cytoskeleton of the cell is known to vary with cell attachment [Titushkin, 2006].

Significant research in microfluidics focuses on chemical and biological detection techniques [Kraly, 2009], [Kim, 2009], [Yi, 2006]. These techniques can be integrated with the $\mu$ PIVOT to identify different molecule or ion concentrations and to analyze the biochemical response of the cell to mechanical stimuli.

\subsection{SUMMARY}

The $\mu$ PIVOT is an instrument combining dual optical tweezers (OT) and micron resolution particle image velocimetry ( $\mu$ PIV). Combined with microfluidics, it is a novel tool to study single cell biomechanics. Cells may be subjected to three dimensional stress fields applied in sequence or simultaneously by stretching of the cell with the dual optical tweezers, compression through hydrostatic pressure, and shear, compression, and extension from uniform or extensional flows. The initial studies indicate 1) a dead cell deforms globally more than a viable cell and presents less resistance to internal organelle rearrangement, 2 ) at the typical laser power (30 $\mathrm{mW}$ at the sample) cell photodamage is negligible for at least 20 minutes while at maximum laser powers ( $1 \mathrm{~W}$ at the sample) photodamage is observed after $\sim 20$ seconds with cell death occurring after 35 seconds, 3) for uniform flows, the maximum fluid induced shear stresses are limited by cell damage to $\sim 15 \mathrm{mPa}$ which is 60 times less than cell monolayer studies, 4) for extensional flow in a microfluidic cross junction, shear stresses of $250 \mathrm{mPa}$ were achieved and substantially greater shear stresses may be applied to suspended cells by 
automation of trap positioning and active control of the cell position relative to the stagnation point, and 5) while osteoblasts show no deformation in extensional flow for shear stresses up to $250 \mathrm{mPa}$, a myoblast is easily deformed in an extensional flow and exhibits a low extension rate and high extension rate deformation regime.

With the $\mu$ PIVOT and microfluidics global and/or local stresses may be applied to a cell without physical contact allowing a new realm of tests to be performed in vitro at the single cell level. This realm of tests may provide novel information on the mechanical response of cells to mechanical stimuli. Coupled with chemical and biological sensors, the $\mu$ PIVOT and microfluidics may bring us closer to understanding the biochemical responses of single cells to mechanical stimuli and the role of physical attachment in the mechanotransduction mechanism. 


\section{CHAPTER 8}

\section{APPLIED FLUID STRESSES IN UNIFORM AND EXTENSIONAL FLOWS}

With advancements in microscale fabrication, microfluidic devices create opportunities to study dynamic mechanical behavior of individual cells under controlled conditions. With fluid mechanics, flow-based mechanical test sequences (including shear and extensional loading) may provide control of unique microenvironments when coupled with single cell suspension techniques. The $\mu$ PIVOT allows us to apply controlled multiaxial stresses to single cells suspended with optical tweezers within custom channel designs. This chapter examines the theoretical stresses applied to suspended cell-sized spheres in uniform and planar extensional flow fields. The calculated fluid stresses indicate limitations in applying fluid-induced stresses in uniform flows and the potentially larger stresses in extensional flows.

\subsection{CALCULATING FLUID STRESSES}

Figure 89 provides a schematic of the coordinate systems used to describe a sphere suspended in a flow field. For both uniform and planar extensional flows, the $\mu$ PIV measurement plane is in the $\varphi=0$ or $x-y$ measurement plane. Assuming Newtonian behavior (shear stress proportional to the strain rate) of the suspending fluid, the normal $\left(\sigma_{r}\right)$ and shear $\left(\tau_{r}\right)$ stresses in the $\mu$ PIV measurement plane are 


$$
\begin{gathered}
\sigma_{r}=-p+\tau_{r r}=-p+2 \mu\left(\frac{\partial u_{r}}{\partial r}\right) \\
\tau_{r \theta}=\mu\left[r \frac{\partial}{\partial r}\left(\frac{u_{\theta}}{r}\right)+\frac{1}{r} \frac{\partial u_{r}}{\partial \theta}\right]
\end{gathered}
$$

where $p$ is the hydrodynamic pressure, $\mu$ is the viscosity of the suspending fluid, $u_{r}$ is the velocity component in the radial direction, and $u_{\theta}$ is the velocity in the $\theta$ direction [Bird, 1960], [White, 1974], [Leal, 2007]. Thus, the stresses imposed on a cell due to local fluid flow can be determined directly from either experimentally measured or theoretically derived velocity fields by calculating radial and transverse velocity gradients.

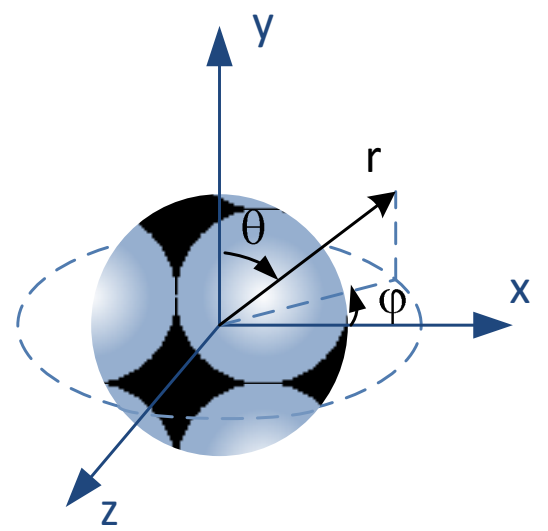

Figure 89: The Cartesian coordinate system was established at the center of the fixed cell position and converted to spherical polar coordinates during stress analysis. For all experiments the flow is planar in the $x-y$ plane $\left(\varphi=0^{\circ}\right)$. 


\subsection{UNIFORM FLOW}

Figure 90a provides a schematic of a spherical cell of radius $a$ suspended in a uniform flow.

For creeping flow conditions (Reynolds number, $\operatorname{Re}<10^{-2}$ ) a freestream velocity $U_{\infty}$ and the boundary conditions $u_{r}=u_{\theta}=0$ and $u \rightarrow U_{\infty}$ at $r \rightarrow \infty$, the Stokes solution for creeping motion past a sphere is [Papanastasiou, 2000], [Leal, 2007], [White, 1974]:

$$
\left\{\begin{array}{l}
u_{r}=U_{\infty}\left(1-\frac{3}{2}\left(\frac{a}{r}\right)+\frac{1}{2}\left(\frac{a}{r}\right)^{3}\right) \cos \theta \\
u_{\theta}=-U_{\infty}\left(1-\frac{3}{4}\left(\frac{a}{r}\right)-\frac{1}{4}\left(\frac{a}{r}\right)^{3}\right) \sin \theta
\end{array}\right.
$$

With these velocities the theoretical normal and shear stresses [Bird, 1960] are

$$
\left\{\begin{array}{c}
\sigma_{r}=-P_{\infty}-\frac{3}{2} \frac{\mu U_{\infty}}{a}\left(\frac{a}{r}\right)^{2} \cos \theta \\
\tau_{r \theta}=\frac{3}{2} \frac{\mu U_{\infty}}{a}\left(\frac{a}{r}\right)^{4} \sin \theta
\end{array}\right.
$$

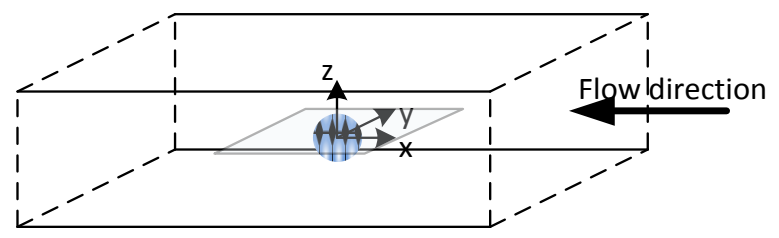

(a)

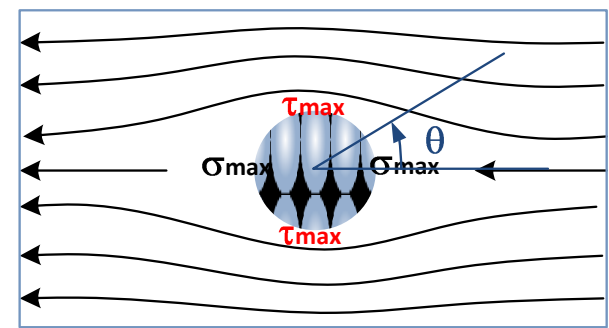

(b)

Figure 90: (a) Schematics of the uniform flow field around a suspended cell. The plane is zoomed in in (b), where the arrows represent the general flow directions. The maximum normal stress $\left(\sigma_{\max }\right)$ is on the flow axis $\left(0^{\circ}\right.$ and $\left.180^{\circ}\right)$ and the maximum shear stress $\left(\tau_{\max }\right)$ is at $90^{\circ}$ and $270^{\circ}$. 
Figure 91 shows the normal stress (left) and shear stress (right) as a function of the angle $\theta$ for several dimensionless distances $(r / a)$ from the sphere surface $(r / a=1$ is at the surface of the sphere). Figure $90 \mathrm{~b}$ shows maximum stress locations around the surface relative to the uniform flow direction. Figure 91 clearly shows the sinusoidal hydrodynamic stresses produce local variations in the shear and normal stresses with the maximum normal stress occurring at $\theta=0^{\circ}$ and $180^{\circ}$ where the shear stress is zero and the maximum shear stress occurring at $\theta=90^{\circ}$ and $270^{\circ}$ where the normal stress is zero. Figure 92 shows the radial dependence of the local maximum normal stresses $(\theta=$ $0^{\circ}$ ) and shear stresses $\left(\right.$ at $\theta=90^{\circ}$ ). Both stresses are a maximum at the sphere surface and decrease rapidly with distance from the sphere.

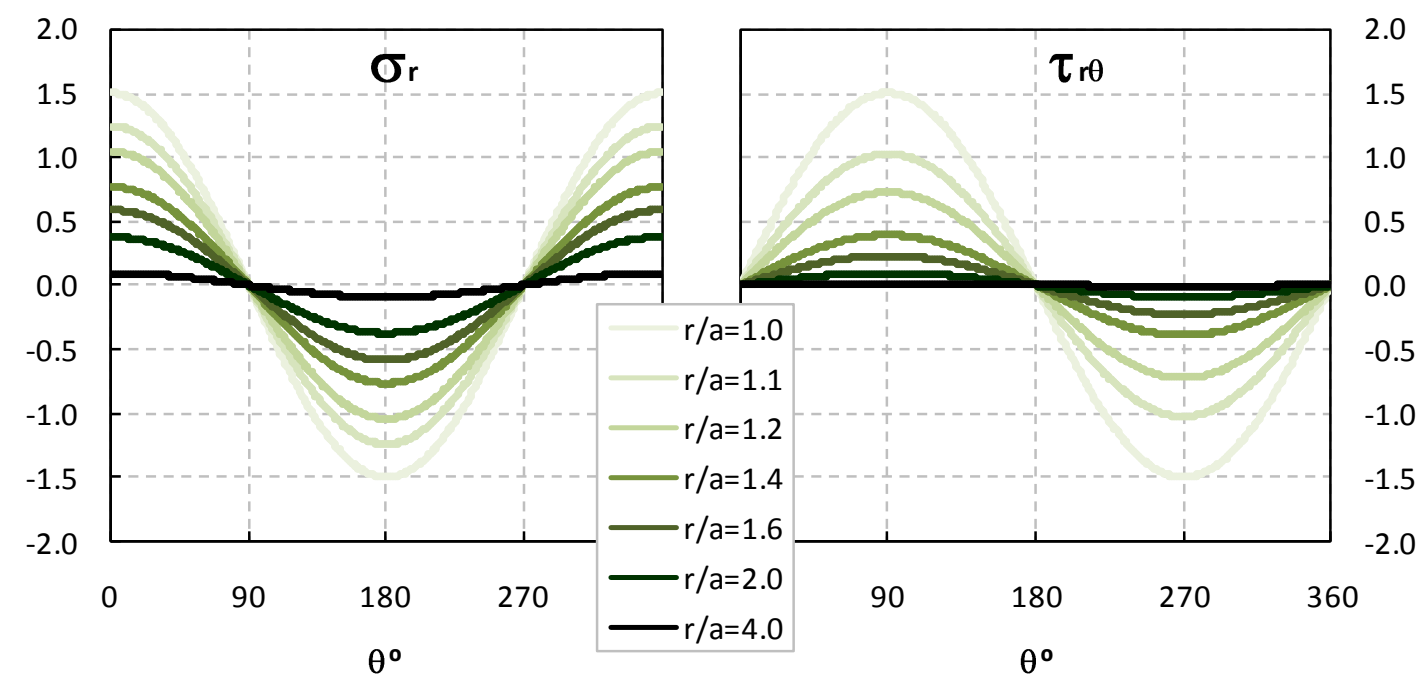

Figure 91: Normalized normal $\left(\sigma_{\mathrm{r}}\right)$ and shear stress $\left(\tau_{\mathrm{r} \theta}\right)$ behavior as a function of angular position around a sphere in uniform flow, $r / a$ is the dimensionless distance from the center of the sphere. 


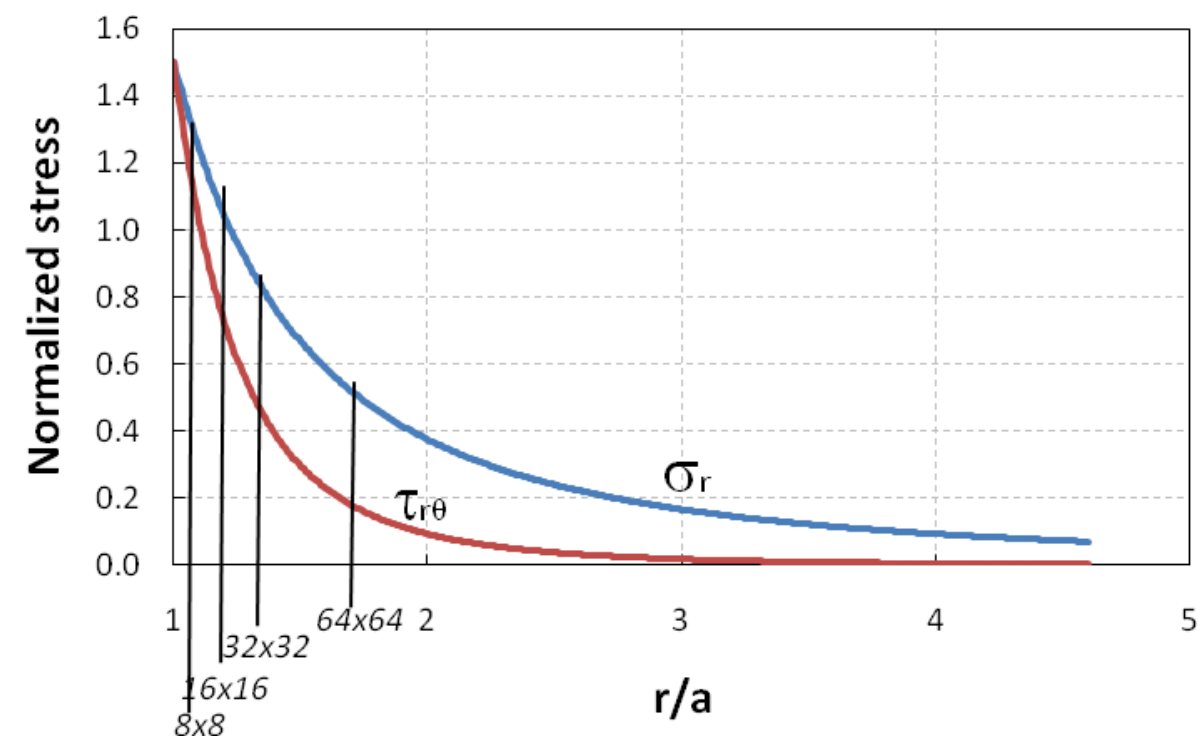

Figure 92: For a sphere in a uniform flow, normalized shear and normal stresses at the angle of maximum stress as a function of distance from the cell surface. The numbers in italic represent the interrogation areas in pixels for $\mu$ PIV measurements.

For practicable purposes, the shear stress reaches the undisturbed flow value within three radii from the cell surface while the normal stress reaches the undisturbed flow value around 5 radii from the cell surface.

A goal in developing the $\mu$ PIVOT is to measure stresses near the surface of the cell. With $\mu$ PIV, the accuracy of velocity measurements is a function of the interrogation size. In Figure 93 the corresponding measurement locations for different interrogation regions are shown. For a typical size cell (radius of $10 \mu \mathrm{m}$ ) a relatively high resolution interrogation region of $8 \times 8$ pixels produces a stress measurement at a radial location of $r / a=1.09$. This results in a normal stress measurement that is $13 \%$ less than the 
maximum normal stress at the surface and a shear stress measurement that is $37 \%$ less than the maximum shear stress at the surface. Applying larger interrogation regions only magnifies this error. Thus, it appears $\mu \mathrm{PIV}$ is insufficient to measure surface stresses unless single pixel interrogation regions (a current $\mu$ PIV research thrust) are used. However, actual stress measurements at the surface are not truly necessary. As shown, the $\mu$ PIV measurements have sufficient resolution to capture the disturbance flow dynamics. The disturbance flow is uniquely a function of the surface boundary conditions, fluid properties, and the undisturbed flow field. Thus, with knowledge of the undisturbed flow field and measurements that capture the disturbance flow dynamics, actual stress conditions at the cell surface can be determined. For uniform flow, current $\mu \mathrm{PIV}$ resolution is sufficient to capture the disturbance flow.

As discussed in Chapter 7, a cell's biological response to imposed stresses depends on both the magnitude and frequency of the applied stress. Previous work on cell monolayers has shown biological responses occurring for shear stresses on the order of $1 \mathrm{~Pa}$ [Donahue, 2003], [Mullender, 2006], [You, 2001], [Johnson, 1996]. Neglecting the hydrostatic contribution (setting $P_{\infty}=0$ ), the maximum hydrodynamic stresses on a sphere suspended in uniform flow are

$$
\sigma_{r, \max }=\tau_{r \theta, \max }=\frac{3}{2} \frac{\mu U_{\infty}}{a}
$$


In our experiments, the uniform flow field was generated by moving an automated stage at constant velocity. The stage was controlled to execute displacement rates up to $500 \mu \mathrm{m} / \mathrm{s}$. With this velocity and a sphere radius, $a$, of $10 \mu \mathrm{m}$, the maximum hydrodynamic stresses applied to the sphere surface are approximately $75 \mathrm{mPa}$. This is over two orders of magnitude less than shear monolayer studies.

Additionally, the sphere is trapped at a depth of 1.5 times the sphere diameter from the bottom coverslip. The upper coverslip is $\sim 1 \mathrm{~mm}$ from the suspended sphere. Thus, the local hydrostatic pressure, $P_{\infty}=\rho g h=9.8 \mathrm{~Pa}$. Therefore, the imposed hydrodynamic stresses are not just smaller than stresses applicable in shear monolayer studies but are even substantially smaller than the hydrostatic pressure.

To achieve hydrodynamic stresses that are comparable to shear monolayer studies and/or equivalent to hydrostatic pressure, the undisturbed velocity must be increased by at least two orders of magnitude. However, increasing the velocity increases the drag on the trapped object. The drag force on a sphere in uniform flow can be obtained by integrating the hydrodynamic stresses in the direction of flow. This yields the classic sphere-drag formula of Stokes

$$
F_{d r a g}=2 \pi \mu U_{\infty} a+4 \pi \mu U_{\infty} a=6 \pi \mu U_{\infty} a
$$

Where $2 \pi \mu U_{\infty} a$ is the form drag and $4 \pi \mu U_{\infty} a$ is the friction drag. In fact, this equation was used to calibrate optical trapping power by equating the trap force to the imposed 
fluid drag (see previous chapters). Under unidirectional flow conditions and the absence of other external forces, the trapping force $F_{O T}$ is statically balanced with the fluid drag force on the cell

$$
F_{O T}=F_{d r a g}=6 \pi \mu U_{\infty} a
$$

Thus, increasing the velocity is not realistic as the drag force increases proportionally with velocity and the trap force is limited to a few hundred picoNewtons. Therefore, the maximum velocity is limited to $400 \mu \mathrm{m} / \mathrm{s}$ by the trap force. As stated earlier, planar extensional flow potentially alleviates this problem.

\subsection{PLANAR EXTENSIONAL FLOW}

In a pure, two-dimensional extensional flow around a sphere there is no flow in the out-of-plane direction ( $z$ direction) and the flow is inward toward the sphere along the $\pm y$ direction and outward away from the sphere in the $\pm x$ direction (see Figure 93a).

For a non-rotating sphere suspended in a general linear flow, the fluid velocity field vector $(\boldsymbol{u})$ is [Leal, 1992]:

$$
\boldsymbol{u}=\boldsymbol{E} \cdot \boldsymbol{x}\left[1-\left(\frac{a}{r}\right)^{5}\right]+\frac{1}{2}(\boldsymbol{\omega} \wedge \boldsymbol{x})\left(1-\frac{a^{3}}{r^{3}}\right)-\boldsymbol{x}(\boldsymbol{x} \cdot \boldsymbol{E} \cdot \boldsymbol{x})\left[\frac{5}{2} \frac{a^{3}}{r^{5}}-\frac{5}{2} \frac{a^{5}}{r^{7}}\right]
$$




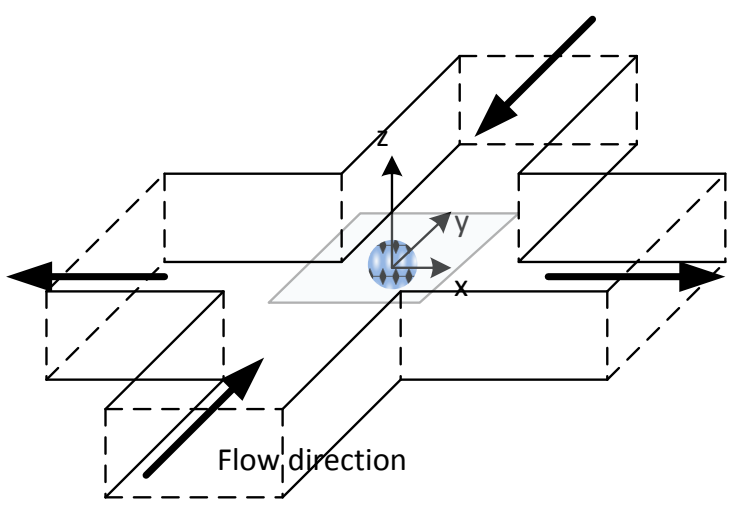

(a)

(b)

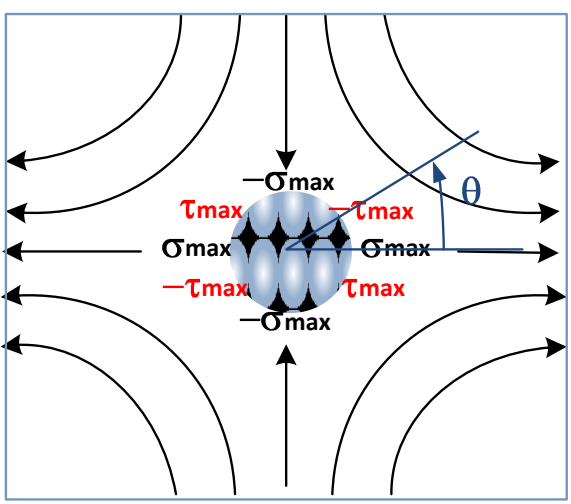

Figure 93: (a) Schematics of the planar extensional flow field around a suspended cell placed at the stagnation point.(b) Magnified view of the light blue plane in (a). The arrows represent the general flow directions. The maximum normal stress $\left(\sigma_{\max }\right)$ is along the inflow and outflow axis and the maximum shear stress $\left(\tau_{\max }\right)$ is offset by $45^{\circ}$.

where $a$ is the sphere radius, $r$ is the radial directional component, $\boldsymbol{x}$ is the position

tensor equal to $\boldsymbol{x}=\left[\begin{array}{l}x \boldsymbol{e}_{\boldsymbol{x}} \\ y \boldsymbol{e}_{\boldsymbol{y}} \\ z \boldsymbol{e}_{z}\end{array}\right]$ in Cartesian coordinates, $\boldsymbol{E}$ is the strain rate tensor, and $\boldsymbol{\omega}$ is the vorticity tensor. For the specific linear case of planar extensional flow the vorticity tensor is identically equal to zero and the rate of strain tensor is

$$
\boldsymbol{E}=\left[\begin{array}{ccc}
\dot{\gamma} & 0 & 0 \\
0 & -\dot{\gamma} & 0 \\
0 & 0 & 0
\end{array}\right]
$$

where $\dot{\gamma}$ is the extension rate. Calculating each component of Equation 8,

$$
\boldsymbol{E} \cdot \boldsymbol{x}=\left[\begin{array}{ccc}
\dot{\gamma} & 0 & 0 \\
0 & -\dot{\gamma} & 0 \\
0 & 0 & 0
\end{array}\right]\left[\begin{array}{c}
x \boldsymbol{e}_{\boldsymbol{x}} \\
y \boldsymbol{e}_{\boldsymbol{y}} \\
z \boldsymbol{e}_{z}
\end{array}\right]=\left[\begin{array}{c}
\dot{\gamma} x \boldsymbol{e}_{x} \\
-\dot{\gamma} y \boldsymbol{e}_{\boldsymbol{y}} \\
0
\end{array}\right]
$$


and

$$
\boldsymbol{x} \cdot \boldsymbol{E} \cdot \boldsymbol{x}=\left[\begin{array}{lll}
x \boldsymbol{e}_{\boldsymbol{x}} & y \boldsymbol{e}_{\boldsymbol{y}} & z \boldsymbol{e}_{z}
\end{array}\right]\left[\begin{array}{c}
\dot{\gamma} x \boldsymbol{e}_{\boldsymbol{x}} \\
-\dot{\gamma} y \boldsymbol{e}_{\boldsymbol{y}} \\
0
\end{array}\right]=\dot{\gamma} x^{2}-\dot{\gamma} y^{2}
$$

Because the object of interest is a sphere, we convert Cartesian to spherical coordinates

by

$$
\left\{\begin{array}{l}
x=r \sin \theta \cos \varphi \\
y=r \sin \theta \sin \varphi \\
z=r \cos \theta
\end{array}\right.
$$

Substituting into Equations 9, 10 and 11:

$$
\begin{gathered}
\boldsymbol{x}=\left[\begin{array}{c}
r \sin \theta \cos \varphi \boldsymbol{e}_{\boldsymbol{x}} \\
r \sin \theta \sin \varphi \boldsymbol{e}_{\boldsymbol{y}} \\
r \cos \theta \boldsymbol{e}_{\boldsymbol{z}}
\end{array}\right] \\
\boldsymbol{E} \cdot \boldsymbol{x}=\left[\begin{array}{c}
\dot{\gamma} r \sin \theta \cos \varphi \boldsymbol{e}_{\boldsymbol{x}} \\
-\dot{\gamma} r \sin \theta \sin \varphi \boldsymbol{e}_{\boldsymbol{y}} \\
0
\end{array}\right] \\
\boldsymbol{x} \cdot \boldsymbol{E} \cdot \boldsymbol{x}=\dot{\gamma} r^{2} \sin ^{2} \theta \cos ^{2} \varphi-\dot{\gamma} r^{2} \sin ^{2} \theta \sin ^{2} \varphi
\end{gathered}
$$

Additionally, the spherical unit vectors and Cartesian unit vectors are related by:

$$
\left[\begin{array}{l}
\boldsymbol{e}_{\boldsymbol{x}} \\
\boldsymbol{e}_{\boldsymbol{y}} \\
\boldsymbol{e}_{\boldsymbol{z}}
\end{array}\right]=\left[\begin{array}{ccc}
\sin \theta \cos \varphi & \cos \theta \cos \varphi & -\sin \varphi \\
\sin \theta \sin \varphi & \cos \theta \sin \varphi & \cos \varphi \\
\cos \theta & -\sin \theta & 0
\end{array}\right]\left[\begin{array}{l}
\boldsymbol{i}_{\boldsymbol{r}} \\
\boldsymbol{i}_{\boldsymbol{\theta}} \\
\boldsymbol{i}_{\boldsymbol{\varphi}}
\end{array}\right]
$$

Which is equivalent to 


$$
\left\{\begin{array}{l}
\boldsymbol{e}_{\boldsymbol{x}}=\sin \theta \cos \varphi \boldsymbol{i}_{\boldsymbol{r}}+\cos \theta \cos \varphi \boldsymbol{i}_{\boldsymbol{\theta}}-\sin \varphi \boldsymbol{i}_{\boldsymbol{\varphi}} \\
\boldsymbol{e}_{\boldsymbol{y}}=\sin \theta \sin \varphi \boldsymbol{i}_{r}+\cos \theta \sin \varphi \boldsymbol{i}_{\boldsymbol{\theta}}+\cos \varphi \boldsymbol{i}_{\boldsymbol{\varphi}} \\
\boldsymbol{e}_{\boldsymbol{z}}=\cos \theta \boldsymbol{i}_{\boldsymbol{r}}-\sin \theta \boldsymbol{i}_{\boldsymbol{\theta}}
\end{array}\right.
$$

Therefore when substituting in Equations 13, 14 and 15, we obtain:

$$
\boldsymbol{x}=\left[\begin{array}{lll}
\boldsymbol{i}_{\boldsymbol{r}} & \boldsymbol{i}_{\boldsymbol{\theta}} & \boldsymbol{i}_{\boldsymbol{\varphi}}
\end{array}\right]\left[\begin{array}{c}
r \sin ^{2} \theta\left(\cos ^{2} \varphi+\sin ^{2} \varphi\right)+r \cos ^{2} \theta \\
r \sin \theta \cos \theta\left(\cos ^{2} \varphi+\sin ^{2} \varphi\right)-r \cos \theta \sin \theta \\
-r \sin \theta \sin \varphi \cos \varphi+r \sin \theta \sin \varphi \cos \varphi
\end{array}\right]=\left[\begin{array}{c}
r \boldsymbol{i}_{r} \\
0 \\
0
\end{array}\right]
$$

$$
\begin{aligned}
\boldsymbol{E} \cdot \boldsymbol{x} & =\left[\begin{array}{lll}
\boldsymbol{i}_{\boldsymbol{r}} & \boldsymbol{i}_{\boldsymbol{\theta}} & \boldsymbol{i}_{\boldsymbol{\varphi}}
\end{array}\right]\left[\begin{array}{ccc}
\sin \theta \cos \varphi & \sin \theta \sin \varphi & \cos \theta \\
\cos \theta \cos \varphi & \cos \theta \sin \varphi & -\sin \theta \\
-\sin \varphi & \cos \varphi & 0
\end{array}\right]\left[\begin{array}{c}
\dot{\gamma} r \sin \theta \cos \varphi \\
-\dot{\gamma} r \sin \theta \sin \varphi \\
0
\end{array}\right] \\
& =\left[\begin{array}{lll}
\boldsymbol{i}_{\boldsymbol{r}} & \boldsymbol{i}_{\boldsymbol{\theta}} & \boldsymbol{i}_{\boldsymbol{\varphi}}
\end{array}\right]\left[\begin{array}{c}
\dot{\gamma} r \sin ^{2} \theta\left(\cos ^{2} \varphi-\sin ^{2} \varphi\right) \\
\dot{\gamma} r \sin \theta \cos \theta\left(\cos ^{2} \varphi-\sin ^{2} \varphi\right) \\
-2 \dot{\gamma} r \sin \theta \sin \varphi \cos \varphi
\end{array}\right] \\
& =\left[\begin{array}{c}
\dot{\gamma} r \sin ^{2} \theta\left(\cos ^{2} \varphi-\sin ^{2} \varphi\right) \boldsymbol{i}_{r} \\
\dot{\gamma} r \sin \theta \cos \theta\left(\cos ^{2} \varphi-\sin ^{2} \varphi\right) \boldsymbol{i}_{\boldsymbol{\theta}} \\
-2 \dot{\gamma} r \sin \theta \sin \varphi \cos \varphi \boldsymbol{i}_{\boldsymbol{\varphi}}
\end{array}\right]
\end{aligned}
$$

And

$$
\boldsymbol{x} \cdot \boldsymbol{E} \cdot \boldsymbol{x}=\dot{\gamma} r^{2} \sin ^{2} \theta \cos ^{2} \varphi-\dot{\gamma} r^{2} \sin ^{2} \theta \sin ^{2} \varphi
$$

So

$$
\boldsymbol{x}(\boldsymbol{x} \cdot \boldsymbol{E} \cdot \boldsymbol{x})=\left[\begin{array}{c}
r \boldsymbol{i}_{\boldsymbol{r}} \\
0 \\
0
\end{array}\right] \dot{\gamma} r \sin ^{2} \theta\left(\cos ^{2} \varphi-\sin ^{2} \varphi\right)
$$




$$
=\left[\begin{array}{c}
\dot{\gamma} r^{2} \sin ^{2} \theta\left(\cos ^{2} \varphi-\sin ^{2} \varphi\right) \boldsymbol{i}_{r} \\
0 \\
0
\end{array}\right]
$$

Expressing the velocity vector in spherical coordinates:

$$
\begin{gathered}
\boldsymbol{u}=\left[\begin{array}{c}
\dot{\gamma} r \sin ^{2} \theta\left(\cos ^{2} \varphi-\sin ^{2} \varphi\right) \boldsymbol{t}_{r} \\
\dot{\gamma} r \sin \theta \cos \theta\left(\cos ^{2} \varphi-\sin ^{2} \varphi\right) \boldsymbol{t}_{\boldsymbol{\theta}} \\
-2 \dot{\gamma} r \sin \theta \sin \varphi \cos \varphi \boldsymbol{\iota}_{\varphi}
\end{array}\right]\left[1-\left(\frac{a}{r}\right)^{5}\right] \\
-\left[\begin{array}{c}
\dot{\gamma} r^{2} \sin ^{2} \theta\left(\cos ^{2} \varphi-\sin ^{2} \varphi\right) \boldsymbol{i}_{r} \\
0 \\
0
\end{array}\right]\left(\frac{5}{2} \frac{a^{3}}{r^{5}}-\frac{5}{2} \frac{a^{5}}{r^{7}}\right)
\end{gathered}
$$

The velocity components are:

$$
\left\{\begin{array}{l}
u_{r}=\left[\dot{\gamma} r \sin ^{2} \theta\left(\cos ^{2} \varphi-\sin ^{2} \varphi\right)\right]\left[1-\left(\frac{a}{r}\right)^{5}\right]-\dot{\gamma} r^{2} \sin ^{2} \theta\left(\cos ^{2} \varphi-\sin ^{2} \varphi\right)\left(\frac{5}{2} \frac{a^{3}}{r^{5}}-\frac{5}{2} \frac{a^{5}}{r^{7}}\right) \\
u_{\theta}=\left[\dot{\gamma} r \sin \theta \cos \theta\left(\cos ^{2} \varphi-\sin ^{2} \varphi\right)\right]\left[1-\left(\frac{a}{r}\right)^{5}\right] \\
u_{\varphi}=[-2 \dot{\gamma} r \sin \theta \sin \varphi \cos \varphi]\left[1-\left(\frac{a}{r}\right)^{5}\right]
\end{array}\right.
$$

Simplifying,

$$
\left\{\begin{array}{l}
u_{r}=\left[\dot{\gamma} r \sin ^{2} \theta\left(\cos ^{2} \varphi-\sin ^{2} \varphi\right)\right]\left[1-\frac{5}{2}\left(\frac{a}{r}\right)^{3}+\frac{3}{2}\left(\frac{a}{r}\right)^{5}\right] \\
u_{\theta}=\left[\dot{\gamma} r \sin \theta \cos \theta\left(\cos ^{2} \varphi-\sin ^{2} \varphi\right)\right]\left[1-\left(\frac{a}{r}\right)^{5}\right] \\
u_{\varphi}=[-2 \dot{\gamma} r \sin \theta \sin \varphi \cos \varphi]\left[1-\left(\frac{a}{r}\right)^{5}\right]
\end{array}\right.
$$


Our $\mu$ PIV measurements are conducted in a planar extensional flow at the centerline.

Thus $\varphi=0$. Additionally, with basic trigonometry, $2 \sin \theta \cos \theta=\sin 2 \theta$ and $2 \sin ^{2} \theta=$ $1-\cos 2 \theta$, the velocity components become

$$
\left\{\begin{array}{l}
u_{r}=\dot{\gamma} r\left[1-\frac{5}{2}\left(\frac{a}{r}\right)^{3}+\frac{3}{2}\left(\frac{a}{r}\right)^{5}\right] \cos 2 \theta \\
u_{\theta}=\dot{\gamma} r\left[1-\left(\frac{a}{r}\right)^{5}\right] \sin 2 \theta \\
u_{\varphi}=0
\end{array}\right.
$$

With the velocities known, the stress tensor components $\left(\tau_{r \theta}\right.$ and $\left.\tau_{r r}\right)$ can be computed from equations (1) and (2).

Substituting in the velocity field,

$$
\left\{\begin{array}{l}
\tau_{r \theta}=-\mu \dot{\gamma} \sin 2 \theta\left[2-5\left(\frac{a}{r}\right)^{3}+8\left(\frac{a}{r}\right)^{5}\right] \\
\tau_{r r}=2 \mu \dot{\gamma} \cos 2 \theta\left[1+5\left(\frac{a}{r}\right)^{3}-6\left(\frac{a}{r}\right)^{5}\right]
\end{array}\right.
$$

Figure 94 shows $\tau_{r r}$ (left) and $\tau_{r \theta}$ (right) as a function of the angle $\theta$ for several dimensionless distances from the sphere surface $(r / a)$. 


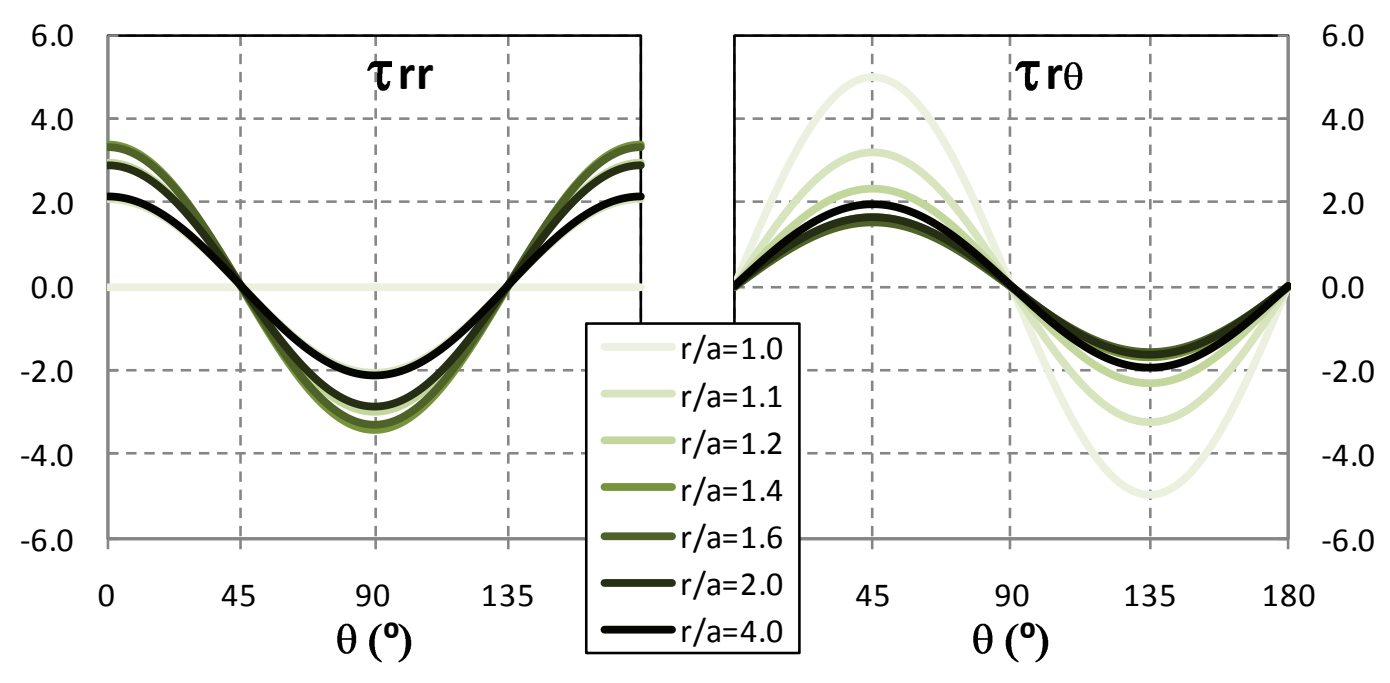

Figure 94: For a planar extensional flow, normalized normal stress without the pressure field $\left(\tau_{\mathrm{rr}}\right)$ and shear stress $\left(\tau_{\mathrm{r} \theta}\right)$ values as a function of the angle from the horizontal $\theta$ for several values of dimensionless distance from the center of the sphere $(r / a)$.

As with uniform flows, the locations of maximum $\tau_{r \theta}\left(\theta=45^{\circ}\right.$ and $\left.135^{\circ}\right)$ correspond to minimum $\tau_{r r}$, and maximum $\tau_{r r}\left(\theta=0^{\circ}\right.$ and $\left.90^{\circ}\right)$ correspond to minimum $\tau_{r \theta}$ (see Figure 93b for maximum locations along the sphere relative to the inflow axis).

However, the cell surface also experiences pressure that cannot be computed from the velocity vectors, hence they cannot be calculated from $\mu$ PIV measurements. The normal stress is the sum of the hydrodynamic pressure and $\tau_{r r}$. The pressure field is equal to

$$
\begin{gathered}
p=-5 \mu(\boldsymbol{x} \cdot \boldsymbol{E} \cdot \boldsymbol{x}) \frac{a^{3}}{r^{5}}+P_{\infty}=-5 \mu \dot{\gamma} \sin ^{2} \theta\left(\frac{a}{r}\right)^{3}+P_{\infty} \\
p=-\frac{5}{2} \mu \dot{\gamma}(1-\cos 2 \theta)\left(\frac{a}{r}\right)^{3}+P_{\infty}
\end{gathered}
$$


Therefore the total normal stress can be computed as the sum of the pressure field and

$\tau_{r r}:$

$$
\sigma_{r}=-P_{\infty}+\frac{5}{2} \mu \dot{\gamma}\left(\frac{a}{r}\right)^{3}+\mu \dot{\gamma} \cos 2 \theta\left[2+\frac{15}{2}\left(\frac{a}{r}\right)^{3}-12\left(\frac{a}{r}\right)^{5}\right]
$$

Figure 95 shows the radial dependence of the local maximum $\tau_{r r}$, pressure field $p$, and the resulting normal stresses $\sigma_{r},\left(\right.$ at $\left.\theta=0^{\circ}\right)$ as well as the maximum shear stresses $\tau_{r} \theta$ (at $\theta=45^{\circ}$ ). The cell experiences a resulting normal stress at its surface which is due completely to the hydrodynamic pressure. Unfortunately the pressure cannot be measured. However, $\tau_{r r}$ can be calculated from the fluid velocity measurements (i.e. normal stress without the pressure field).

Figure 95 shows the curves of $\tau_{r r}$ and $\sigma_{r}$ have a similar profile with a fast increase of the stress moving away from the cell surface, and then a gradual decrease to reach the undisturbed flow value of 2 , at approximately 4 radii away from the cell surface. While the maximum shear stress also reaches the undisturbed flow value of 2 at approximately 4 radii from the sphere, a minimum shear stress below the undisturbed value occurs at $r / a=1.63$. 


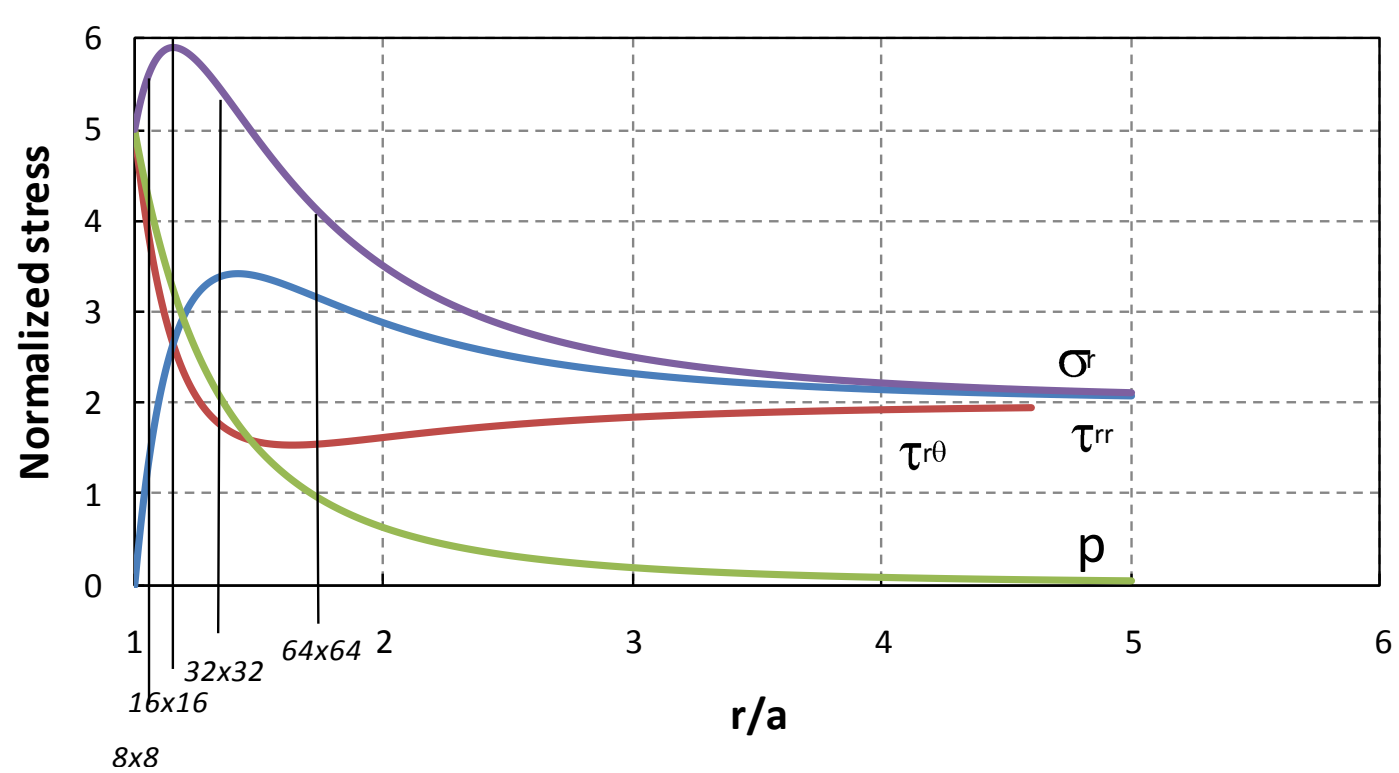

Figure 95: Normalized shear and normal stresses at the angle of maximum stress as a function of distance from the cell surface. The numbers in italic represent the interrogation areas in pixels for $\mu$ PIV measurements.

As with uniform flow, deciphering the disturbance flow associated with flow around a spherical particle in a planar extensional flow requires sufficiently small $\mu$ PIV interrogation regions to map velocities near the cell surface. In Figure 95 the corresponding measurement locations for different interrogation regions are shown. Compared to uniform flow stresses, the rebound of the shear and normal stress curves add additional complications in deciphering the disturbance flow. Specifically, it is not possible to differentiate a measured stress from the stress of the undisturbed flow if the measured shear stress has a value of 2 . Therefore, to accurately measure the stress near a suspended cell and capture the actual disturbance flow generated by the cell, measurements must be made close to the cell. For example, for shear stresses, the limit 
to capture the disturbance flow would be within 0.25 radii from the cell surface, meaning for a $10 \mu \mathrm{m}$ radius cell, velocity measurements should be within $2.5 \mu \mathrm{m}$ of the surface. The 16 pixel $\times 16$ pixel interrogation used in the experiments provides velocity measurements within this range. However, to truly measure stress at the cell surface, dramatically smaller interrogation regions are required. With the current $\mu$ PIV system, a single pixel corresponds to an imaged area of $109 \mathrm{~nm} \times 109 \mathrm{~nm}$. Thus, the single pixel interrogation PIV, technique developed by Westerweel et al. [Westerweel, 2004], is required to truly resolve stresses at the cell surface. However, this may not be necessary. As shown here, the disturbance flow shear stress is resolvable for $r / a<1.1$. An interrogation region of 8 pixels $\times 8$ pixels is within this range for disturbance flow around a $10 \mu \mathrm{m}$ sphere and can be resolved with the current $\mu$ PIV technique. However, for any of the stresses, the disturbance flow is uniquely a function of the surface boundary conditions, fluid properties, and the undisturbed flow field. With knowledge of the undisturbed flow field and measurements that capture the disturbance flow dynamics, actual stress conditions at the cell surface can be determined. Current $\mu$ PIV resolution is sufficient to capture the disturbance flow.

In a planar extensional flow, a cell held at the stagnation point undergoes the two major fluid stresses: hydrostatic pressure and hydrodynamic stresses. Neglecting the hydrostatic contribution (setting $P_{\infty}=0$ ), the maximum hydrodynamic stresses on a sphere suspended in an extensional flow are 


$$
\sigma_{r, \max }=\tau_{r \theta, \max }=5 \mu \dot{\gamma}
$$

Interestingly, the maximum shear stress is independent of size and only depends on the fluid viscosity and extension rate. For our planar extensional flow experiments the maximum normal and shear stresses that we were able to apply to the surface of a cellsized sphere were $250 \mathrm{mPa}$ for the maximum strain rate of $50 \mathrm{~s}^{-1}$. Here again, hydrostatic pressures dominate. To generate the flow, a fluid head of $24.5 \mathrm{~mm}$ was required. This corresponds to a hydrostatic pressure of $119.75 \mathrm{~Pa}$.

Contrarily to uniform flow, if the shear and normal stress are integrated around the surface of a sphere trapped in planar extensional flow, the net fluid drag is identically zero. Thus, the fluid velocities and corresponding strain rate may be increased without an increase in the drag force exerted on the sphere if the sphere is positioned at the stagnation point (center) of the two-dimensional extensional flow. Thus, while hydrodynamic stresses are limited in uniform and straight channel flow scenarios, no such limitation exists for the two-dimensional extensional flow generated in a cross-junction. Theoretically, if a cell is positioned perfectly at the stagnation point, no trapping force is required to maintain its position, regardless of the applied shear rate, effectively creating a hydrodynamic trap. In reality, the stagnation point represents a saddle point and is unstable to perturbations along the $\mathrm{x}$ axis. However, only a small force, much less than the maximum trapping force, is required to maintain the sphere at the stagnation point. For the extensional environment examined, a 
relatively low shear rate (limited by manual positioning of the sphere/cell) was applied. However, the potential exists for dramatically higher hydrodynamic stresses (at least 100x greater than reported). In addition, the dominant nature of the hydrostatic stress state may be mitigated by proper cross-junction design. Therefore, while the biological effects of hydrodynamic stresses in uniform and straight channel flows around suspended cells appear limited, significant cell deformation and biological responses are at least possible for single cells suspended in cross-junction flows.

\subsection{CONCLUSION}

In this chapter, local normal and shear fluid stresses applied to suspended microspheres in uniform and extensional flows were calculated in order to determine the magnitude of hydrodynamic stresses experienced by single cells suspended in fluidic environments and more specifically to clarify the performance of the $\mu$ PIVOT for single cell analysis.

Applied shear and normal stresses were calculated for the theoretical flow velocity data in the region surrounding the surface perimeter of a typical size spherical non-deformable cell (radius $a=10 \mu \mathrm{m}$ ). The advantage in the presented line of research is the opportunity to assess isolated cell biomechanics with eventual correlations to mechanotransduction and diseases with or without cellular adhesion responses. With 
improved resolution, applied stresses to cell membrane structures and the elastic membrane itself can be assessed in a detailed stress versus strain response.

While hydrodynamic stresses are limited in uniform and straight channel flow scenarios, no such limitation exists for the two-dimensional extensional flow generated in a cross-junction. For the extensional environment described here, a relatively low shear rate was examined. However, the potential exists for dramatically higher hydrodynamic stresses (at least 100x greater than reported). In addition, the dominant nature of the hydrostatic stress state may be mitigated by proper cross-junction design. Therefore, while the biological effects of hydrodynamic stresses in uniform and straight channel flows around suspended cells appear limited, significant cell deformation and biological responses are possible for single cells suspended in cross-junction flows. 


\section{CHAPTER 9}

\section{SUMMARY AND FUTURE WORK}

\subsection{SUMMARY}

A novel instrument has been developed by integrating two laser-based techniques: micron-resolution particle image velocimetry ( $\mu \mathrm{PIV})$ and optical tweezers (OT) within a single microscope. Optical tweezers allow the capture, suspension, and manipulation of polystyrene and glass microspheres, as well as single cells or biomolecules by optical gradient forces. $\mu$ PIV can measure local fluid movements including that of steady uniform and extensional flows in addition to providing high resolution imaging of cellular deformation. The $\mu$ PIVOT maintains the individual capabilities of $\mu$ PIV and OT. When applied simultaneously, interference between the two techniques is limited to a region of influence around the trap position. Without a trapped micron-sized object, a localized dynamic nanoparticle accumulation and release in the vicinity of the OT was observed. Nevertheless, outside this region of influence, the agglomerating process had no measurable effect on velocity measurements in the image plane. For flow analysis with a micron-sized trapped object, the clustering process was observed to generally be negligible, and OT and $\mu$ PIV could be used simultaneously without noticeable interaction. In order to validate the integrated techniques, we compared computational predictions to the measured velocity profile 
around a trapped particle in either an imposed microchannel flow or a uniform flow. Good quantitative agreement between measured and predicted velocities was observed for $15 \mu \mathrm{m}$ to $35 \mu \mathrm{m}$ diameter trapped particles subjected to fluid velocities from 50 $\mu \mathrm{m} / \mathrm{s}$ to $500 \mu \mathrm{m} / \mathrm{s}$ even at the highest laser power (1.45 W). Additionally, this validation demonstrated the first reported full field velocity measurements around a freely suspended particle in a unidirectional flow. These measurements were performed with the particle being several radii away from a wall (the coverslip). Direct comparison with computational models showed strong quantitative agreement. When compared to theoretical results for a particle in an infinite fluid, the results indicate the $\mu$ PIVOT is capable of resolving wall effects due to the presence of the coverslip.

As a final validation step, the Optical Trap stiffness was characterized using polystyrene spheres. This parameter depends not only on the laser, but also on the surrounding medium and the trapped object. The trap stiffness was shown to be inversely proportional to the sphere diameter $\mathrm{k}=\mathrm{C} / \mathrm{d}$, and independent of depth to several radii away from the coverslip.

The $\mu$ PIVOT was then applied to study the interaction between two microspheres in a uniform flow. The spheres were positioned at different distances from each other with their centerline at varying angles from the flow axis. For this low Reynolds number flow, the drag forces of leading and trailing sphere were equal for any 
distance or angle. Compared to computational models, the results agreed qualitatively. Further experiments are necessary to confirm the quantitative agreement. After successful validation, the major application combined the $\mu$ PIVOT with microfluidics to study single cell biomechanics. Using this novel arrangement, cells were subjected to three dimensional stress fields applied in sequence or simultaneously. Cells were stretched with the dual optical tweezers and compressed through hydrostatic pressure. Shear, compressional, and extensional stresses were imparted on a cell surface by uniform or extensional flows. The initial studies indicate that cells deform significantly in uniform flows, but the maximum fluid induced shear stresses were limited by cell damage to $\sim 15 \mathrm{mPa}$ which is 60 times less than for cell monolayer studies. For extensional flow in a microfluidic cross junction, shear stresses of $250 \mathrm{mPa}$ were achieved. At this extension rate, a myoblast was shown to be easily deformed and exhibited a low and high extension rate deformation regime.

Additionally, the optical trap system characteristics were exploited to differentiate cells. Specifically, the trap stiffness was calculated for all cells tested in uniform flows. As stated above, this parameter depends not only on the optical trap system but on the trapped object as well. Therefore, for identical trap system settings, the trap stiffness depends solely on the trapped object (i.e. the cell). By Comparing the trap stiffness results for cells of different type (bone or cartilage cells), passage, surface roughness, protrusions size and number, processing method, and culture flask 
concentration, the chondroblast results showed that two cell variables seemed to affect the trap stiffness value significantly: 1 ) the presence of small cilia at the surface of the cell and 2) the cell processing method between flask extraction and experiments.

\subsection{FUTURE WORK}

\subsubsection{INSTRUMENTATION IMPROVEMENT}

A system with greater automation for more efficient experimental protocols and "on the fly" object positioning is necessary. Towards this goal, a LabView program (National Instruments Corp., Austin, TX) is being created and will be available shortly. It enables the upload of predetermined trap positions or trajectories, stage velocities, and many other automated features. It will dramatically increase the efficiency of the experiments as well as shorten the post-processing time. The LabView program will also provide the means to better measurements: presently the automated stage velocity can be set to a constantvalue. However for a variable velocity, the joystick is used and no read out is possible. Acquiring a live readout will allow us to increase the velocity continuously while monitoring its value. A direct improvement using this feature is the possibility to precisely measure the trapping efficiency from the maximum drag force imparted on a trapped object just before it escapes.

Another necessary improvement is the addition of other methods to measure the trap stiffness. This parameter is a key to any experimental results using the optical 
tweezers. However its determination is empirical and its accuracy is essential. As stated in Chapter 2, the trap stiffness can be measured using several other methods than the drag force method:

- In the equipartition method, the Brownian motion of the particle is measured while trapped. Knowing the temperature $(T)$ and measuring the average of the object displacement $(\Delta x)$, the trap stiffness is equal to $k=\frac{k_{B} T}{<\Delta x^{2}>}$, with $k_{B}$, the Boltzmann constant.

- In the power spectrum method, the Brownian motion is also used to calculate the trap stiffness. Here, the trap stiffness is directly proportional to the corner frequency. This corner frequency is obtained by calculating the Fourier transform of the particle motion $\Delta x(t)$ and fitting a Lorentzian to the data. We can then graph the power spectrum of the particle motion and obtain the corner frequency.

- In the step response method, the trap is moved rapidly. The particle then returns to the center of the trap exponentially in time. Knowing the drag coefficient, the measurement of the rate constant of this exponential leads to the calculation of the trap stiffness.

Automation of the imaging system and stage control makes the power spectrum and step response methods viable. 
Another concern is the current biological incompatibility of $\mu$ PIV with biological cells. Specifically, the $\mu$ PIV nanoparticles are preserved in a solution containing Azide, which is strongly toxic. No commercially available particles suitable for our experiments exist without the preservative. When $\mu$ PIV particles are introduced in a cell solution, the cell dies rapidly and the $\mu$ PIV particles clump. This prevents any $\mu$ PIV experiments from being conducted. This apparent problem can be resolved by either custom ordering fluorescent nanoparticles (at a substantial cost) or filtering the nanoparticles in a dialysis system prior to an experiment.

\subsubsection{FUTURE APPLICATIONS USING THE $\mu$ PIVOT}

\subsubsection{Biological cell applications}

For the biological cell experiments, many enhancements can be added to the protocol. One major improvement would be the measurement of the biochemical response of the cell to mechanical stimuli. Previous studies have shown that bone or cartilage cells' first reaction to mechanical loading is the increase in intracellular calcium

concentration $\left[\mathrm{Ca}^{2+}\right]$ [Roberts, 2001] and the production of nitric oxide (NO) [Fink, 2001]. Using the white light of the confocal microscope, many fluorescent studies are accessible, and marked $\left[\mathrm{Ca}^{2+}\right]$ or Nitrite could be detected by our instrumentation. Using this new feature, we could for example compare the cell's response to different flows or 
mechanical stimuli when anchored to a surface or freely suspended. This application would add knowledge to the process of mechanotransduction.

\subsubsection{Fluid dynamics and particles interactions}

This work represents the first time flow has been characterized around a freely suspended object. Thus, with this novel instrumentation, unique studies on fluid-particle interactions at the microscale are now possible. Theoretical models that have been in textbooks for years can now be confirmed including wall effects associated with microspheres in fluid flows. The interaction between two suspended spheres in flow can be studied more extensively with $\mu$ PIV including the recirculation flow patterns that develop due to the spheres rotation. More importantly, the ability to simultaneously measure the velocity field and drag force enables a unique combination of local and global stress measurements. This combination could elucidate subtle differences in computational models of non-Newtonian fluids where an "incorrect" predicted local stress field can generate a "correct" predicted drag force.

\subsection{CONCLUSION}

The outcomes of the described work lay the foundation for future work in particle-

fluid hydrodynamics and cell biomechanics. The capabilities enabled by the $\mu$ PIVOT may provide insight into fluid-particle hydrodynamics, non-Newtonian fluid mechanics, the mechanical response of cells, the mechanotransduction process, the understanding of 
cell states within the same tissue, and the design of engineered biomaterials which control cellular function. 


\section{REFERENCES}

Aberts, B., Bray, D., Lewis, L., Raff, M., Roberts, K., \& Watson, J. D. (1994). Molecular biology of the cell. 3rd ed. New York: Garland.

Afoke, N. Y., Byers, P. D., \& Hutton, W. C. (1987). Contact pressures in the human hip joint. J Bone Joint Surg Br. , 69, 536-541.

Akeson, W. H., Amiel, D., Abel, M. F., Garfin, S. R., \& Woo, S. L. (1987). Effects of immobilization on joints. Clin. Orthop. , 219, 28-37.

Alexopoulos, L. G., Haider, M. A., Vail, T. P., \& Guilak, F. (2003). Alterations in the mechanical properties of the human chondrocyte pericellular matrix with osteoarthritis. J. Biomech. Eng. (Trans. ASME) , 125 (3), 323-333.

Alexopoulos, L. G., Williams, G. M., Upton, M. L., Setton, L. A., \& Guilak, F. (2005). Osteoarthritic changes in the biphasic mechanical properties of the chondrocyte pericellular matrix in articular cartilage. J. Biomech. , 38 (3), 509-517.

Asbury, C. L., Fehr, A. N., \& Block, S. M. (2003). Kinesin Moves by an Asymmetric HandOver-Hand Mechanism. Science, 302 (5653), 2130.

Ashkin, A. (1992). Forces of a single-beam gradient laser trap on a dielectric sphere in the ray optics regime. Biophysical Journal , 61 (2), 569-582.

Ashkin, A., \& Dziedzic, J. M. (1987). Optical trapping and manipulation of viruses and bacteria. Science , 235 (4795), 1517-1520.

Ashkin, A., Dziedzic, J. M., \& Yamane, T. (1987). Optical trapping and manipulation of single cells using infrared-laser beams. Nature , 330 (6150), 769-771.

Bachrach, N. M., Valhmu, W. B., Stazzone, E., Ractcliffe, A., Lai, W. M., \& Mow, V. C. (1995). Changes in proteoglycan synthesis of chondrocytes in articular cartilage are associated with the time-dependent changes in their mechanical environment. J. Biomech , 28, 1561-1569.

Bao, S., \& Suresh, G. (2003). Cell and molecular mechanics of biological materials. Nature Materials , 2 (11), 715-725. 
Bentley, B. J., \& Leal, L. G. (1986a). A computer-controlled four-roll mill for investigations of particle and drop dynamics in two-dimensional linear shear flows. $J$. Fluid Mech. , 167, 219-240.

Bentley, B. J., \& Leal, L. G. (1986b). An experimental investigation of drop deformation and break-up in steady, two-dimensional linear flows. J. Fluid Mech. , 167, 241-283.

Binnig, G., Quate, C. F., \& Gerber, C. (1986). Atomic force microscope. Phys. Rev. Lett. , 56, 930-933.

Bird, R. B., Stewart, W. E., \& Lightfoot, E. N. (1960). Transport Phenomena. NY: John Wiley \& Sons.

Bourret, L. A., \& Rodan, G. A. (1976). The role of calcium in the inhibition of cAMP accumulation in epiphyseal cartilage cells exposed to physiological pressure. J. Cell Physio. , 88 (3), 353-362.

Buckwalter, J. A., Martin, J. A., \& Brown, T. D. (2006). Perspectives on chondrocyte mechanobiology and osteoarthritis. Biorheology , 43, 603-609.

Bull, B., Feo, C., \& Bessis, M. (1983). Behavior of Elliptocytes under Shear Stress in the Rheoscope and Ektacytometer. Cytometry, 3 (4), 300-304.

Bull, B., Feo, C., \& Bessis, M. (1983). Behavior of Elliptocytes under Shear Stress in the Rheoscope and Ektacytometer. Cytometry, 3 (4), 300-304.

Burton-Wurster, N., Vernier-Singer, M., Farquhar, T., \& Lust, G. (1993). Effect of compressive loading and unloading on the synthesis of total protein, proteoglycan, and fibronectin by canine cartilage explants. J. Orthop. Res. , 11, 717-729.

Buschmann, M. D., Gluzband, Y. A., Grodzinsky, A. J., \& Hunziker, E. B. (1995). Mechanical compression modulates matrix biosynthesis in chondrocyte/agarose culture. J. Cell Sci. , 108, 1497-1508.

Buschmann, M. D., Hunziker, E. B., Kim, Y. J., \& Grodzinsky, A. J. (1996). Altered aggrecan synthesis correlates with cell and nucleus structure in statically compressed cartilage. J. Cell Sci. , 109, 499-508. 
Cerf, A., Cau, J. C., Vieu, C., \& Dague, E. (2009). Nanomechanical properties of dead or alive single-patterned bacteria. Langmuir , 25 (10), 5731-5736.

Chaudhuri, O., Parekh, S. H., Lam, W. A., \& Fletcher, D. A. (2009). Combined atomic force microscopy and side-view optical imaging for mechanical studies of cells. Nature Methods , 6, 383-387.

Chen, N. X., Ryder, K. D., Pavalko, F. M., Turner, C. H., Burr, D. B., Qiu, J., et al. (2000). $\mathrm{Ca} 2+$ regulates fluid shear-induced cytoskeletal reorganization and gene expression in osteoblasts. Am J Physiol Cell Physiol , 278, C989-C997.

Chen, R. C., \& Lu, Y. N. (1999). The flow characteristics of an interactive particle at low Reynolds numbers. Int. J. Multiphase Flow, 25, 1645-1655.

Chen, R. C., \& Wu, J. L. (2000). The flow characteristics between two interactive spheres. Chemical engineering Science, 55 (6), 1143.

Cook, R. D., \& Young, W. C. (1985). Advanced Mechanics of Materials. NY: Macmillan Publishing Company.

Costa, K. (2006). Imaging and probing cell mechanical properties with the atomic force microscope. Methods in Molecular Biology , 319, 331-361.

Cross, S. E., Jin, Y. S., Rao, J., \& Gimzewski, J. K. (2007). Nanomechanical analysis of cells from cancer patients. Nature Nanotechnol. , 2, 780-783.

Darling, E. M., Zauscher, S., \& Guilak, F. (2006). Viscoelastic properties of zonal articular chondrocytes measured by atomic force microscopy. OsteoArthritis and Cartilage , 14, 571-579.

Declercq, H., Van den Vreken, N., De Maeyer, E., Verbeeck, R., Schacht, E., De Ridder, L., et al. (2004). Isolation, proliferation and differentiation of osteoblastic cells to study cell/biomaterial interactions: comparison of different isolation techniques and source. $25,757-768$.

Dietrick, J. E., Whedon, G., \& Shorr, E. (1948). Effects of immobilization upon various metabolic and physiological functions of bone. Am. Jnl. Med. , 4, 3-36. 
Donahue, T. L., Haut, T. R., Yellowey, C. E., Donahue, H. J., \& Jacobs, C. R. (2003). Mechanosensitivity of bone cells to oscillating fluid flow induced shear stress may be modulated by chemotransport. J Biomech , 36, 1363-1371.

Eggleton, C. D., Pawar, Y. P., \& Stebe, k. J. (1999). Insoluble surfactants on a drop in an extensional flow: a generalization of the stagnated surface limit to deforming interfaces. J. Fluid Mech. , 385, 79-99.

Ehrlich, M. G., Armstrong, A. L., Treadwell, B. V., \& Mankin, H. J. (1987). The role of proteases in the pathogenesis of osteoarthritis. J Rheumatol. , 14, 30-32.

Evander, M., Johansson, L., Lilliehorn, T., Piskur, J., Lindvall, M., Johansson, S., et al. (2007). Noninvasive acoustic cell trapping in a microfluidic perfusion system for online bioassays. Anal Chem. , 79 (7), 2984-2991.

Evans, E., \& Yeung, A. (1989). Apparent viscosity and cortical tension of blood granulocytes determined by micropipet aspiration. Biophys J. , 56 (1), 151-160.

Evans, E., \& Yeung, A. (1989). Apparent viscosity and cortical tension of blood granulocytes determined by micropipet aspiration. Biophys J. , 56 (1), 151-160.

Eveson, G. F., Hall, E. W., \& Ward, S. G. (1959). Interaction between two equal-sized equal-settling spheres moving through a viscous liquid. Brit. J. Appl. Phys. , 10, 43-47.

Fallman, O., \& Axner, E. (2003). Influence of a glass-water interface on the on-axis trapping of micrometer-sized spherical objects by optical tweezers. Applied Optics , 42 (19), 3915-3926.

Felder, S., \& Elson, E. L. (1990). Mechanics of fibroblast locomotion: quantitative analysis of forces and motions at the leading lamellas of fibroblasts. J. Cell Biol. , 111, 25132526.

Ferraro, J. T., Daneshmand, M., Bizios, R., \& Rizzo, V. (2004). Depletion of plasma membrane cholesterol dampens hydrostatic pressure and shear stress-induced mechanotransduction pathways in osteoblast cultures. Am J Physiol Cell Physiol. , 286, c831-C839. 
Fink, C., Fermor, B., Weinberg, J. B., Pisetsky, D. S., Misukonis, M. A., \& Guilak, F. (2001). The effect of dynamic mechanical compression on nitric oxide production in the meniscus. Osteoarthritis and Cartilage , 9, 481-487.

Florin, E. L., Horber, J. K., \& Stelzer, E. H. (1996). High-resolution axial and laterial position sensing using two-photon excitation of fluorophores by a continuous-wave Nd/YAG laser. Applied Physics Letter , 69 (4), 446-448.

Florin, E. L., Pralle, A., Horber, J. K., \& Stelzer, E. H. (1997). Photonic force microscope based on optical tweezers and two-photon excitation or biological applications. J. Struct. Biol. , 119, 202-211.

Folkersma, R., Stein, H. N., \& Van de Vosse, F. N. (2000). Hydrodynamic interactions between two identical spheres held fixed side by side against a uniform stream directed perpendicular to the line connecting the spheres' centres. International Journal of Multiphase Flow , 26, 877-887.

Francius, G., Domenech, O., Mingeot-Leclercq, M. P., \& Dufrene, Y. F. (2008). Direct observation of Staphylococcus aureus cell wall digestion by Lysostaphin. J Bacteriol. , 190 (24), 7904-7909.

Ganatos, P., Pfeffer, R., \& Weinbaum, S. (1980). A strong interaction theory for creeping motion of a sphere between plane parallel boundaries. Part2, Parallel motion. J. Fluid Mech, 99 (4), 755-783.

Ghislain, L. P., Switz, N. A., \& Webb, W. W. (1994). Measurement of small forces using an optical trap. Rev. Sci. Instrum. , 65 (9), 2762-2768.

Glantschnig, H., Varga, F., \& Rumpler, M. (1996). Prostacyclin (PGI2): a potential mediator of c-fos expression induced by hydrostatic pressure in osteoblastic cells. Eur J Clin Invest , 26, 533-548.

Gray, M. L., Pizzanelli, A. M., Grodzinsky, A. J., \& Lee, R. C. (1988). Mechanical and physiochemical determinants of the chondrocyte biosynthetic response. J. Orthop. Res. , 6, 777-792. 
Guck, J., Ananthakrishnan, R., Mahmood, H., Moon, T. J., Cunningham, C. C., \& Kas, J. (2001). The Optical Stretcher: A Novel Laser Tool to Micromanipulate Cells. Biophysical Journal , 81 (2), 767-784.

Guilak, F., Alexopoulos, L. G., Haider, M. A., Tring-Beall, H. P., \& Setton, L. A. (2005). Zonal uniformity in mechanical properties of the chondrocyte pericellular matrix: micropipette aspiration of canine chondrons isolated by cartilage homogenization. Ann. Biomed. Eng. , 33 (10), 1312-1318.

Guilak, F., Meyer, C. B., Ratcliffe, A., \& Mow, V. C. (1994). The effects of matrix compression on proteoglycan metabolism in articular cartilage explants. Osteoarthritis Cartilage , 2, 91-101.

Haapala, J., Lammi, M. J., Inkinen, R., Parkkinen, J. J., Agren, U. M., Arokoski, J., et al. (1996). Coordinated regulation of hyaluronan and aggrecan content in the articular cartilage of immobilized and exercised dogs. J. Rheumatol. , 23, 1586-1593.

Happel, J., \& Brenner, H. (1983). Low Reynolds number hydrodynamics. The Hague: Martinus Nijoff.

Happel, J., \& Pfeffer, R. (1960). The motion of two spheres following each other in a viscous fluid. American Institute of Chemical Engineers Journal , 6, 129-133.

Hassan, E., Heinz, W. F., Antonik, M. D., D'Costa, N. P., Nageswaran, S., Schoenenberger, C. A., et al. (1998). Relative microelastic mapping of living cells by atomic force microscopy. Biophys. J. , 74, 1564-1578.

Hayden, H., Kamm, R. D., \& Lee, R. T. (2004). Cell mechanics and mechanotransduction: pathways, probes, and physiology. Am J Physiol Cell Physiol , 287, C1 - C11.

Ho, B. P., \& Leal, L. G. (1974). Inertial migration of rigid spheres in two-dimensional unidirectional flows. J. Fluid Mechanics , 65, 365-400.

Hochmuth, R. M. (2000). Micropipette aspiration of living cells. Journal of Biomechanics., 33 (1), 15-22.

Hochmuth, R. M., \& Waugh, R. E. (1987). Erythrocyte membrane elasticity and viscosity. Annu Rev Physiol. , 49, 209-219. 
Hochmuth, R. M., \& Waugh, R. E. (1987). Erythrocyte membrane elasticity and viscosity. Annu Rev Physiol. , 49, 209-219.

Hochmuth, R. M., \& Waugh, R. E. (1987). Erythrocyte membrane elasticity and viscosity. Annu Rev Physiol. , 49, 209-219.

Hodge, W. A., Carlson, K. L., Fijan, R. S., Burgess, R. G., Riley, P. O., Harris, W. H., et al. (1989). Contact pressures from an instrumented hip endoprosthesis. J Bone Joint Surg Am. , 71, 1378-1386.

Hough, L. A., \& Ou-Yang, H. D. (2002). Correlated motions of two hydrodynamically coupled particles confined in separate quadratic potential wells. Physical Review E , 65, 021906.

Hu, S., Chen, J., Fabry, B., Numaguchi, Y., Gouldstone, A., Ingber, D. E., et al. (2003). Intracellular stress tomography reveals stress focusing and structural anisotropy in cytoskeleton of living cells. Am J Physiol Cell Physiol , 285, C1082-1090.

Huang, H., Kamm, R. D., \& Lee, R. T. (2004). Cell mechanics and mechanotransduction: pathways, probes, and physiology. Am J Physiol Cell Physiol , 287, C1-C11.

Hudson, S. D., Phelan, F. R., Handler, M. D., Cabral, J. T., Migler, K. B., \& Amis, E. J. (2004). Microfluidic analog of the four-roll mill. Applied Physics Letters , 85 (2).

Hunt, T. P., \& Westervelt, R. M. (2006). Dielectrophoresis tweezers for single cell manipulation. Biomed Microdevices , 8 (3), 227-230.

Im, K. B., Kim, H. I., Joo, I. J., Oh, C. H., Song, S. H., Kim, P. S., et al. (2003). Optical trapping forces by a focused beam through two media with different refractive indices. Opt. Commun. , 226, 25-31.

Ingber, D. E. (2003). Tensegrity I. Cell structure and hierarchical systems biology. J. Cell Sci. , 116, 1157-1173.

Ingber, D. E. (1997). Tensegrity: the architectural basis of cellular mechanotransduction. Annu. Rev. Physiol. , 59, 575-599. 
Jaasma, M., Jackson, W., Tang, R., \& Keaveny, T. (2007). Adaptation of cellular mechanical behavior to mechanical loading for osteoblastic cells. Journal of Biomechanics. 40 (9), 1938-1945.

Jin, G., Sah, R. L., Li, Y. S., Lotz, M., Shyy, J. Y., \& Chien, S. (2000). Biomechanical regulation of matrix metalloproteinase-9 in cultured chondrocytes. J Orthop Res. , 18, 899-908.

Johnson, D. L., McAllister, T. N., \& Frangos, J. A. (1996). Fluid flow stimulates rapid and continuous release of nitric oxide in osteoblasts. Am J Physiol Endocrinol Metab , 271, E205-E208.

Jones, E. (1996). Human Cell Culture Protocols, Methods in Molecular Medicine. (Gareth, Ed.) Humana Press.

Jones, W. R., Tring-Beall, H. P., Lee, G. M., Kelley, S. S., Hochmuth, R. M., \& Guilak, F. (1999). Alterations in the Young's Modulus and Volumetric Properties of Chondrocytes Isolated From Normal and Osteoarthritic Human Cartilage. J. Biomech. , 32 (2), 119-127.

Kamm, R. D., \& Kaazempur-Mofrad, M. R. (2004). On the molecular basis for mechanotransduction. Mech. Chem. Biosystems 1, 3, 201-209.

Kaneta, T., Makihara, J., \& Imasaka, T. (2001). An "optical channel": a technique for the evaluation of biological cell elasticity. Analytical chemistry, 73 (24), 5791-5795.

Kapur, S., Baylink, D. J., \& Lau, K. H. (2003). Fluid flow shear stress stimulates human osteoblast proliferation and differentiation through multiple interacting and competing signal transduction pathways. Bone , 32 (3), 241-251.

Keh, H. J., \& Chen, S. H. (1997). Low Reynolds-number hydrodynamic interactions in a suspension of spherical particles with slip surfaces. Chemical Engineering Science , 52 (11), 1789-1805.

Kim, J., Junkin, M., Kim, D. H., Kwon, S., Shin, Y. S., Wong, P. K., et al. (2009). Applications, techniques, and microfluidic interfacing for nanoscale biosensing. Microfluidics and Nanofluidics , 7, 149-167. 
Knoner, G., Parkin, S., Heckenberg, N. R., \& Rubinsztein-Dunlop, H. (2005).

Characterization of optically driven fluid stress fields with optical tweezers. Phys. Rev. E., 72 (3), 031507.

Koay, E. J., Shieh, A. C., \& Athanasiou, K. A. (2003). Creep indentation of single cells. Journal of Biomechanical Engineering , 125 (3), 334-341.

Kohles, S. S., Neve, N., Zimmerman, J. D., \& Tretheway, D. C. (2009). Mechanical Stress Analysis of Microfluidic Environments Designed for Isolated Biological Cell Investigations. Journal of Biomechanical Engineering , 131 (12), 121006 (10 pages).

Kraly, J. R., Holcomb, R. E., Guan, Q., \& Henry, C. S. (2009). Review: Microfluidics applications in metabolomics and metabolic profiling. Analytica Chemica Acta , 653, 2335.

Kuo, S. C., \& Sheetz, M. P. (1992). Optical tweezers in cell biology. Trends Cell Biol. , 2, 116-118.

Kwon, R. Y., \& Jacobs, C. R. (2007). Time-dependent deformations in bone cells exposed to fluid flow in vitro: Investigating the role of cellular deformation in fluid flow-induced signaling. J Biomech. , 40 (14), 3162-3168.

Lang, M., Asbury, C., Shaevitz, J., \& Block, S. M. (2002). An automated two-dimensional optical force clamp for single molecule studies. Biophys. J. , 83, 491-501.

Leal, L. G. (2007). Advanced Transport Phenomena: Fluid Mechanics and Convective Transport Processes. NY: Cambridge University Press.

Leal, L. G. (1992). Laminar flow and convective transport processes: scaling principles and asymptotic analysis. Boston, MA: Butterworth-Heinemann.

Lee, D. A., \& Bader, D. L. (1997). Compressive strains at physiological frequencies influence the metabolism of chondrocytes seeded in agarose. J. Orthop. Res. , 15, 181188.

Lee, G. Y., \& Lim, C. T. (2007). Biomechanics approaches to studying human diseases. Trends Biotechnol. , 25 (3), 111-118. 
Leipzig, N. D., \& Athanasiou, K. A. (2005). Unconfined creep compression of chondrocytes. J Biomech, 38, 77-85.

Leonardo, R. D., Leach, J., Mushfique, H., Cooper, J. M., Ruocco, G., \& Padgett, M. J. (2006). Multipoint holographic optical velocimetry in microfluidic systems. Phys. Rev. Lett. , 96, 134502.

Leyton-Mange, J., Yang, S., Hoskins, M. H., Kunz, R. F., Zahn, J. D., \& Dong, C. (2006). Design of a side-view particle imaging velocimetry flow system for cell-substrate adhesion studies. J Biomech Eng. , 128 (2), 271-278.

Liang, H., Vu, K. T., Krishnan, P., Trang, T. C., Shin, D., Kimel, S., et al. (1996). Wavelength dependence of cell cloning efficiency after optical trapping. Biophys J. , 70, 1529-1533.

Liao, G. B., Bareil, P. B., Sheng, Y., \& Chiou, A. (2008). One-dimensional jumping optical tweezers for optical stretching of bi-concave human red blood cells. Optics Express , 16 (3), 1996-2004.

Liu, Y., Cheng, D., Sonek, G. J., Berns, M. W., Chapman, C. F., \& Tromberg, B. J. (1995). Evidence for localized cell heating induced by infrared optical tweezers. Biophys. J. , 68, 2137-2144.

Liu, Y., Sonek, G. J., Berns, M. W., \& Tromberg, B. J. (1996). Physiological monitoring of optically trapped cells: assessing the effects of confinement by 1064-nm laser tweezers using microfluorometry. Biophys J. , 71, 2158-2167.

Lutz, B. R., Chen, J., \& Schwartz, D. T. (2006). Hydrodynamic tweezers: 1. Noncontact trapping of single cells using steady streaming microeddies. Anal Chem. , 78 (15), 54295435.

Mack, P. B., \& La Chance, P. L. (1967). Effects of recumbency and space flight on bone density. Am. J. Clin. Nutrition, 20, 194-205.

Malagnino, N., Pesce, G., Sassoa, A., \& Arimondo, E. (2002). Measurements of trapping efficiency and stiffness in optical tweezers. Optics Communications , 214, 15-24.

Malone, A. M., Anderson, C. T., Tummala, P., Kwon, R. Y., Johnston, T. R., Stearns, T., et al. (2007). Primary cilia mediate mechanosensing in bone cells. PNAS, 104 (33), 1332513330. 
Mazolli, A., Maia Neto, P. A., \& Nussenzveig, H. M. (2003). Theory of trapping forces in optical tweezers. Proceedings of the Royal Society Lond. A , 459, 3021-3041.

McAllister, T. N., \& Frangos, J. A. (1999). Steady and transient fluid shear stress stimulate NO release in osteoblasts through distinct biochemical pathways. J Bone Miner Res. , 14, 930-936.

Meinhart, C. D., Wereley, S., \& Santiago, J. (2000). A PIV algorithm for estimating timeaveraged velocity fields. J. Fluid Eng. , 122 (2), 285-289.

Melton, L. J., Atkinson, E. J., \& O'Connor, M. K. (1998). Bone density and fracture risk in men. J Bone Miner Res , 13, 1915.

Melton, L. J., Chrischilles, E. A., \& Cooper, C. (1992). Perspective. How many women have osteoporosis? J Bone Miner Res , 7, 1005.

Mikuni-Takagaki, Y. (1999). Mechanical responses and signal transduction pathways in stretched osteocytes. J. Bone Miner. Metab. , 17, 57-60.

Mills, J. P., Qie, L., Dao, M., Lim, C. T., \& Suresh, S. (2004). Nonlinear elastic and viscoelastic deformation of the human red blood cell with optical tweezers. MCB , 1 (3), 169-180.

Morey, E. R., \& Baylink, D. K. (1978). Inhibition of bone formation during space flight. Science, $201,1138-1141$.

Mow, V. C., Kuei, S. C., Lai, W. M., \& Armstrong, C. G. (1980). Biphasic creep and stress relaxation of articular cartilage in compression? Theory and experiments. J. Biomech. Eng. (Trans. ASME) , 102 (1), 73-84.

Mow, V. C., Setton, L. A., Guilak, F., \& Ratcliffe, A. (1995). Mechanical factors in articular cartilage and their role in osteoarthritis. In K. E. Kuettner, \& V. M. Goldberg (Eds.), Osteoarthritis Disorders (pp. 147-171). Rosemont, IL: American Academy of Orthopaedic Surgeons.

Mow, V. C., Wang, C. C., \& Hung, C. T. (1999). The extracellular matrix, interstitial fluid and ions as a mechanical signal transducer in articular cartilage. Osteoarthritis Cartilage, 7, 41-58. 
Mullender, M. G., Dijcks, S. J., Bacabac, R. G., Semeins, C. M., Van Loon, J. J., \& KleinNulend, J. (2006). Release of nitric oxide, but not prostaglandin E2, by bone cells depends on fluid flow frequency. Journal of Orthopaedic Research , 24 (6), 1170-1177.

Muller, F. J., Setton, L. A., Manicourt, D. H., Mow, V. C., Howell, D. S., \& Pita, J. C. (1994). Centrifugal and biochemical comparison of proteoglycan aggregates from articular cartilage in experimental joint disuse and joint instability. J. Orthop. Res. , 12, 498-508.

Neuman, K. C., \& Block, S. M. (2004). Optical trapping. Review of Scientific Instruments, 75 (9), 2787-2809.

Neuman, K. C., Chadd, E. H., Liou, G. F., Bergman, K., \& Block, S. M. (1999).

Characterization of photodamage to Escherichia coli in optical traps. Biophys J. , 77 (5), 2856-2863.

Nève, N., Lingwood, J. K., Zimmerman, J., Kohles, S. S., \& Tretheway, D. C. (2008). The $\mu P I V O T:$ an integrated particle image velocimeter and optical tweezers instrument for microenvironment investigations. Meas. Sci. Technol. , 10 (9), 095403 (11pp).

Nguyen, M., \& Wereley, S. T. (2002). Fundamentals and Applications of Microfluidics. Norwood, MA: Artech Hous.

Oegema, J. T., Lewis, J. L., \& Thompson, J. R. (1993). Role of acute trauma in development of osteoarthritis. Agents Actions , 40, 220-223.

Ofek, G., \& Athanasiou, K. A. (2007). Micromechanical properties of chondrocytes and chondrons: relevance to articular cartilage tissue engineering. Journal of Mechanics of Materials and Structures , 2 (6), 1059-1086.

Olsen, M. G., Bauer, J. M., \& Beebe, D. J. (2000). Particle imaging technique for measuring the deformation rate of hydrogel microstructures. Applied Physics Letters, $76,3310-3312$.

Ozkaya, N., \& Nordin, M. (c1999). Fundamentals of biomechanics: equilibrium, motion, and deformation (Vol. 2nd ed). (D. L. Leger, Ed.) New York: Springer.

Pang, W. L. (2006). PDMS Replica Molding and Microfluidic Device Finishing Revision 1.3. UCSD Systems Biodynamics Laboratories , 7-8. 
Papanastasiou, T. C., Georgio, G. C., \& Alexandrou, A. N. (2000). Viscous Fluid Flow. NY: CRC Press.

Parkkinen, J. J., Ikonen, J., Laman, M. J., Laakonen, J., Tammi, M., \& Helminen, H. J. (1993). Effects of cyclic hydrostatic pressure on proteoglycan synthesis in cultured chondrocytes and articular cartilage explants. Arch Biochem Biophys. , 300, 458-465.

Parkkinen, J. J., Lammi, M. J., Helminen, H. J., \& Tammi, M. (1992). Local stimulation of proteoglycan synthesis in articular cartilage explants by dynamic compression in vitro. J. Orthop. Res , 10, 610-620.

Parkkinen, J. J., Lammi, M. J., Tammi, M. I., \& Helminen, H. J. (1994). Proteoglycan synthesis and cytoskeleton in hydrostatically loaded chondrocytes. In V. C. Mow, F. Guilak, R. Tran-Son-Tray, \& R. M. Hochmuth (Eds.), Cell Mechanics and Cellular Engineering (pp. 420-444). New-York: Springer-Verlag.

Pasternak, C., \& Elson, E. L. (1985). Lymphocyte Mechanical Response Triggered by Cross-Linking Surface Receptors. J. Cell Biol. , 100, 860-872.

Patankar, S. V., \& Spalding, D. B. (1972). A Calculation Procedure for Heat, Mass and Momentum Transfer in Three-Dimensional Parabolic Flows. Int J Heat Mass Transfer, 15, 1787-1806.

Patankar, S. V. (1980). Numerical Heat Transfer and Fluid Flow. Washington, DC: Taylor \& Francis.

Peake, M. A., Cooling, L. M., Magnay, J. L., Thomas, P. B., \& El Haj, A. J. (2000). Selected contribution: regulatory pathways involved in mechanical induction of c-fos gene expression in bone cells. J Appl Physiol. , 89 (6), 2498-2507.

Petersen, N. O., McConnaughey, W. B., \& Elson, E. L. (1982). Dependence of locally measured cellular deformability on position on the cell, temperature, and cytochalasin b. Proc Natl Acad Sci USA, 79, 5327-5331.

Polin, N., Ladavac, K., Lee, S.-H., Roichman, Y., \& Grier, D. (2005). Optimized holographic optical traps. Opt. Exp, 13, 5831-5845.

Radmacher. (2002). Measuring the elastic properties of living cells by the atomic force microscope. Methods Cell Biol. , 68, 67-90. 
Ramaswamy, S., \& Leal, L. G. (1999). The deformation of a viscoelastic drop subjected to steady uniaxial extensional flow of a newtonian fluid. J. Non-Newtonian Fluid Mech. , 85, 127-163.

Reich, K. M., Gay, C. V., \& Frangos, J. A. (1990). Fluid shear stress as a mediator of osteoblast cyclic adenosine monophosphate production. J Cell Physiol. , 143, 100-104.

Roberts, S. R., Knight, M. M., \& Lee, D. A. (2001). Mechanical compression influences intracellular Ca2+ signaling in chondrocytes seeded in agarose constructs. J Appl Physiol, 90, 1385-1391.

Roelofsen, J., Klein-Nulend, J., \& Burger, E. H. (1995). Mechanical stimulation by intermittent hydrostatic compression promotes bone-specific gene expression in vitro. $J$ Biomech , 28, 1493-1503.

Rohrbach, A., \& Stelzer, E. H. (2002). Trapping forces, force constants, and potential depths for dielectric spheres in the presence of spherical aberrations. Applied Optics , 41 (13), 2494-2507.

Roosen, G. (1979). Optical levitation of spheres. Can. J. Phys. , 57, 1260-1279.

Roosen, G., \& Imbert, C. (1976). Optical levitation by means of 2 horizontal laser beamstheoretical and experimental study. Physics. Lett. , 59A, 6-8.

Rotsch, C., \& Radmacher, M. (2000). Drug-induced changes of cytoskeletal structure and mechanics in fibroblasts - An atomic force microscopy study. Biophys. J. , 78, 520-535.

Rotsch, C., \& Radmacher, M. (2000). Drug-induced changes of cytoskeletal structure and mechanics in fibroblasts: An atomic force microscopy study. Biophysical Journal , 78, 520-535.

Sah, R. L., Kim, Y. J., Doong, J. Y., Grodzinsky, A. J., Plaas, A. H., \& Sandy, J. D. (1989). Biosynthetic response of cartilage explants to dynamic compression. J. Orthop. Res. , 7, 619-636.

Santiago, J. G., Wereley, S. T., Meinhart, C. D., Beebe, D. J., \& Adrian, R. J. (1998). A particle image velocimetry system for microfluidics. Exp. Fluids. , 25, 316-319. 
Sato, M., Levesque, M. J., \& Nerem, R. M. (1987). An application of the micropipette technique to the measurement of the mechanical properties of cultured bovine aortic endothelial cells. Journal of Biomechanical Engineering , 109.

Sato, S., Ohyumi, M., Shibata, H., \& Inaba, H. (1991). Optical trapping of small particles using a 1.3-micrometer compact InGaAsp diode laser. Opt. Lett. , 16, 282-284.

Schmid-Schonbein, G. W., Sung, K. L., Tözeren, H., Skalak, R., \& Chien, S. (1981). Passive mechanical properties of human leukocytes. Biophysical Journal , 36 (1), 243-256.

Schmid-Schonbein, H., Wells, R., \& Goldstone, J. (1969). Influence of Deformability of Human Red Cells upon Blood Viscosity. Circ. Res. , 25, 131-143.

Shieh, A. C., \& Athanasiou, K. A. (2002). Biomechanics of single chondrocytes and osteoarthritis. Critical Reviews in Biomedical Engineering , 30 (4-6), 307-343.

Shieh, A. C., \& Athanasiou, K. A. (2003). Principles of cell mechanics for cartilage tissue engineering. Ann. Biomed. Eng. , 31, 1-11.

Shieh, A. C., Koay, E. J., \& Athanasiou, K. A. (2006). Strain-dependent recovery behavior of single chondrocytes. Biomech. Model. Mechanobiol. , 5 (2-3), 172-179.

Shin, A., \& Athanasiou, K. A. (1999). Cytoindentation for obtaining cell biomechanical properties. J. Orthop. Res. , 17, 880-890.

Simmons, R. M., Finer, J. T., Chu, S., \& Spudich, J. A. (1996). Quantitative measurements of force and displacement using an optical trap. Biophys. J. , 70, 1813-1822.

Sipe, J. D. (2002). Tissue engineering and reparative medicine. Ann NY Acad Sci. , 961, 19.

Sleep, J., Wilson, D., Simmons, R., \& Gratzer, W. (1999). Elasticity of the red cell membrane and its relation to hemolytic disorders: an optical tweezers study. Biophys. J., 77, 3085-3095.

Slowman-Kovacs, S. D., Braunstein, E. M., \& Brandt, K. D. (1990). Rapidly progressive Charcot arthropathy following minor joint trauma in patients with diabetic neuropathy. Arthritis Rheum. , 33, 412-417. 
Smith, L. R., Risk, S. F., Ellison, S. F., Wessells, P., Tsuchiya, K., Carter, D. R., et al. (1996). In vitro stimulation of articular chondrocyte mRNA and extracellular matrix synthesis by hydrostatic pressure. J Orthop Res. , 14, 53-60.

Stimson, M., \& Jeffery, G. B. (1926). The motion of two spheres in a viscous fuid. Proc. $R$. Soc. Lond A, 111, 110-116.

Stone, S. W., Meinhart, C. D., \& Wereley, S. T. (2002). A microfluidic-based nanoscope. Experiments in Fluids , 33 (5), 613-619.

Su, S. S., \& Schmid-Schonbein, G. W. (2008). Fluid stresses on the membrane of migrating leukocytes. Ann Biomed Eng. , 36 (2), 298-307.

Sunk, I. G., Trattnig, S., Graninger, W. B., Amoyo, L., Tuerk, B., Steiner, C. W., et al. (2006). Impairment of chondrocyte biosynthetic activity by exposure to 3-tesla high-field magnetic resonance imaging is temporary. Arthritis Res Ther. , 8 (4), R106.

Svoboda, K., \& Block, S. M. (1994). Biological applications of optical forces. Ann. Rev. Biophys. Biomol. Struct. , 23, 247-285.

Tan, S. C., Pan, W. X., Ma, G., Cai, N., Leong, K. W., \& Liao, K. (2008). Viscoelastic behaviour of human mesenchymal stem cells. BMC Cell Biology , 9, 40 (7 pages).

Theret, D. P., Levesque, M. J., Sato, M., Nerem, R. M., \& Weeler, L. T. (1988). The application of a homogeneous half-space model in the analysis of endothelial cell micropipette measurements. J. Biomech. Eng. (Trans. ASME) , 110 (3), 190-199.

Thompson, J. R., Vener, M. J., Griffiths, H. J., Lewis, J. L., Oegema, J. T., \& Wallace, L. (1993). Scanning electron-microscopic and magnetic resonance-imaging studies of injuries to the patellofemoral joint after acute transarticular loading. J. Bone Joint Surg. Am. , 75, 704-713.

Thoumine, O., Ott, A., Cardoso, O., \& Meister, J.-J. (1999). Microplates: a new tool for manipulation and mechanical perturbation of individual cells. J. Biochem. Biophys. Methods , 39, 47-62.

Titushkin, I., \& Cho, M. (2006). Distinct membrane mechanical properties of human mesenchymal stem cells determined using laser optical tweezers. Biophyisical Journal, 90, 2582-2591. 
Toyoda, T., Seedhom, B. B., Yao, J. Q., Kirkham, J., Brookes, S., \& Bonass, W. A. (2003). Hydrostatic pressure modulates proteoglycan metabolism in chondrocytes seeded in agarose. Arthritis Rheum. , 48 (10), 2865-2872.

Tretheway, D. C., \& Leal, L. G. (2001). Deformation and relaxation of Newtonian drops in planar extensional flows of a Boger Fluid. J. Non-Newtonian Fluid Mech. , 99, 81-108.

Tretheway, D. C., \& Leal, L. G. (1999). Surfactant and viscoelastic effects on drop deformation in 2-D extensional flow. AlChE Journal , 45 (5), 929-937.

Tretheway, D. C., \& Meinhart, C. D. (2002). Apparent fluid slip at hydrophobic microchannel walls. Phys. Fluids, 14, L9-12.

Trickey, W. R., Lee, G. M., \& Guilak, F. (2000). Viscoelastic properties of chondrocytes from normal and osteoarthritic human cartilage. J. Orthopaed. Res. , 18 (6), 891-898.

Valhmu, W. B., Stazzone, E. J., Bachrach, N. M., Saed-Nejad, F., Fischer, S. G., Mow, V. C., et al. (1998). Load-controlled compression of articular cartilage induces a transient stimulation of aggrecan gene expression. Arch. Biochem. Biophys. , 353, 29-36.

Vermeulen, K. C., Wuite, G. J., Stienen, G. J., \& Schmidt, C. F. (2006). Optical trap stiffness in the presence and absence of spherical aberrations. Applied Optics , 45 (8), 1812-1819.

Visscher, K., Gross, S. P., \& Block, S. M. (1996). Construction of multiple-beam optical traps with nanometer resolution position sensing. IEEE J. Sel. Topics Quantum. Electron., 2, 1066-1076.

Walker, L. M., Holm, A., Cooling, L., Maxwell, L., Oberg, A., Sundqvist, T., et al. (1999). Mechanical manipulation of bone and cartilage cells with optical tweezers. FEBS Lett. , $459,39-42$.

Wei, M. T., Zaorski, A., Yalcin, H. C., Wang, J., Hallow, M., Ghadiali, S. N., et al. (2008). A comparative study of living cell micromechanical properties by oscillatory optical tweezers. Optics Express, 16, 8594-8603.

Wereley, S. T., Gui, L. C., \& Meinhart, C. D. (Jan 2001). Flow Measurement Techniques for the Microfrontier. American Institute of Aeronautics and Astronautics Annual Meeting, (pp. Paper 2001-0243). Reno, NV. 
Wereley, S., Meinhart, C., Gui, L., Tretheway, D. C., \& Sud, A. (2005). Single pixel evaluation of microchannel flows. Proceedings of the IMECE, (p. paper \#83065).

Westerweel, J., Geelhoed, P. F., \& Lindken, R. (2004). Single-pixel resolution ensemble correlation for micro-PIV applications. Exp Fluids. , 37, 375-384.

White, F. M. (1974). In Viscous Fluid Flow (p. 123). New-York: McGraw-Hill.

Wilkes, R. P., \& Athanasiou, K. A. (1996). The intrinsic incompressibility of osteoblast-like cells. Tissue Engineering , 2 (3), 167-181.

Williams, M. C. (2002). Optical tweezers: measuring picoNewton forces. In P. Schwille (Ed.), Single Molecule Techniques. Bethesda, MD: Biophysical Society.

Wu, L., Tsutahara, M., Kim, L., \& Ha, M. (2008). Numerical simulations of droplet formation in a cross-junction microchannel by the lattice Boltzmann method. Int.J. Numer. Meth. Fluids, 57, 793-810.

Xia, Y., \& Whitesides, G. M. (1998). Soft lithography. Annual reviews of materials sciences, 28, 153-184.

Yang, C. H., Huang, K. S., Lin, P. W., \& Lin, Y. C. (2007). Using a cross-flow microfluidic chip and external crosslinking reaction for monodisperse TPP-chitosan microparticles. Sensors and Actuators B: Chemical , 124 (2), 510-516.

Yi, C. Q., Li, C. W., Ji, S. L., \& Yang, M. S. (2006). Microfluidics technology for manipulation and analysis of biological cells. Analytical Chimica Acta , 560, 1-23.

Yoon, D.-H., \& Yang, K.-S. (2007). Flow-induced forces on two nearby spheres. Physics of Fluids, 19 (9), 098103.

You, J., Reilly, G. C., Zhen, X., Yellowley, C. E., Chen, Q., Donahue, H. J., et al. (2001). Osteopontin gene regulation by oscillatory fluid flow via intracellular calcium mobilization and activation of mitogen-activated protein kinase in MC3T3-E1 osteoblasts. J. Biol Chem , 13365-13371.

Zamir, E., \& Geiger, B. (2001). Molecular complexity and dynamics of cell-matrix adhesions. Journal of Cell Science, 114 (20), 3583-3590. 
Zhang, Q. Y., Wang, X. H., Wei, X. C., \& Chen, W. Y. (2008). Characterization of viscoelastic properties of normal and osteoarthritic chondrocytes in experimental rabbit model. Osteoarthritis Cartilage , 16 (7), 837-840. 